\title{
Laser sintering of Si-Ge nanoparticles for thermoelectric materials
}

\author{
A Dissertation \\ Presented to \\ the faculty of the School of Engineering and Applied Science \\ University of Virginia
}

In Partial Fulfillment of the requirements for the Degree of

Doctor of Philosophy in Electrical Engineering

by

Tyson Baldridge

December 2012 


\section{Approval Sheet}

This dissertation is submitted in partial fulfillment of the

requirements for the degree of

Doctor of Philosophy in Electrical Engineering

Tyson Baldridge

This dissertation has been read and approved by the examining committee:

\section{Prof. Mool C. Gupta}

Dissertation Advisor

Prof. Lloyd R. Harriott

Chair

Prof. Joe C. Campbell

Prof. S. Joseph Poon

Prof. Patrick E. Hopkins

Accepted for the School of Engineering and Applied Science:

Prof. James H. Aylor

Dean, School of Engineering and Applied Sciences

December 2012 


\section{Abstract}

Thermoelectric materials directly convert heat into electricity by generating a voltage from a temperature differential, without any moving parts or noise. Improving the efficiency of thermoelectric materials is important to advancing clean energy generation, especially given current US policy and societal views on reducing oil consumption and generation of greenhouse gases. Select automotive manufacturers have demonstrated improved miles per gallon (MPG) when using thermoelectric generators in test vehicles, however performance needs to be enhanced to facilitate market penetration.

Thermoelectric material performance is described by the dimensionless figure of merit "ZT", where $\mathrm{Z}$ is the figure of merit and $\mathrm{T}$ is the average temperature. The material performance is proportional to 1) thermopower squared and inversely proportional to 2) electrical resistivity and 3) thermal conductivity. The goal is to produce a phonon-glass electron-crystal (PGEC), in other words to maintain high electrical conductivity yet keep heat transfer low. Recently, much research to increase ZT has focused on reducing thermal conductivity via nanostructuring.

In this work, we investigate laser sintering of thin films of doped silicon-germanium nanoparticles using a Continuous-Wave $(\mathrm{CW})$ diode laser for higher performance based on nanostructuring and lower cost. Si-Ge is non-toxic, stable at temperatures up to $1000{ }^{\circ} \mathrm{C}$, and can be doped both $\mathrm{n}$ - and $\mathrm{p}$ - type. Laser processing allows extremely fast heating and cooling rates, which in turn minimizes the amount of nanoparticle size growth and hence generates a large density of interfaces to reduce thermal transport by phonons. By using nanoclusters with a starting grain size of 5-9 $\mathrm{nm}$ prior to laser sintering, a bottom-up approach is taken for achieving thermoelectric films. Thermopower and electrical resistivity are characterized using an Ulvac ZEM-3, and Time-Domain ThermoReflectance (TDTR) is used to measure the thermal conductivity of the sintered layer. Morphology and composition are analyzed using a Scanning Electron Microscope (SEM) and Energy Dispersive x-ray Spectroscopy (EDS). HighTemperature X-Ray Diffraction (HT-XRD) is used for structural characterization of the raw and laser sintered films.

Our laser sintering method achieved one of the lowest reported thermal conductivity values for $\mathrm{Si}-\mathrm{Ge}$ of $1.36 \mathrm{~W} / \mathrm{m} / \mathrm{K}$, near the Si-Ge amorphous limit of $\sim 1 \mathrm{~W} / \mathrm{m} / \mathrm{K}$ at room 
temperature, when sintering on fused silica substrate. Using silicon substrate, a high thermopower and good electrical conductivity were measured, where the absolute Seebeck coefficient exceeded $300 \mu \mathrm{V} / \mathrm{K}$ and electrical conductivity measured as $2.4 * 10^{4} \mathrm{~S} / \mathrm{m}(0.004$ $\Omega \cdot \mathrm{cm}$ ) at room temperature. The peak power factor of $0.0029 \mathrm{~W} / \mathrm{m} / \mathrm{K}^{2}$ occurred at $600 \mathrm{~K}$. Due to sample inhomogeneity over the measurement volumes between thermal and electrical measurements, exact ZT values could not be calculated. The peak ZT value is expected to be near 0.1 due to high thermal conductivity of the Si substrate, which reduces efficiency to $~ 2 \%$ given a 300 to $1000 \mathrm{~K}$ operating range. High-temperature XRD confirmed the sintered materials are stable to temperatures $>1000{ }^{\circ} \mathrm{C}$.

Laser processing has the potential for cost reduction and facilitates processing simplicity via flexibility with both materials and geometries. This work serves as the foundation for $\mathrm{CW}$ laser sintering of Si-Ge thermoelectrics, a stepping stone to other thermoelectric materials, and the possibility of enhanced thermoelectric performance for recovery of waste heat worldwide. 


\section{Acknowledgment}

All of this research was made possible by my advisor Prof. Mool C. Gupta, for which I am forever grateful. His guidance and encouragement has enabled me to learn the intricacies of problem solving without being stymied by the myriad of obstacles involved with designing and testing fundamental research in the laboratory. Prof. Gupta has an uncanny ability to uncover the critical nuances of a problem while maintaining perspective on achieving a solution to the original goal.

I would like to thank the NASA Langley Professor Program, the Air Force Office of Scientific Research, AREVA, Inc., and ORNL User Agreement NP-10-0323 for financial support. I am sincerely grateful to Dan Fekto in the ECE department for his administrative support of the financial side of research, and both David Rowe and Prof. Uwe Kortshagen at the University of Minnesota for working with us to provide materials otherwise unobtainable. Similarly, I'm indebted to Ramez Cheaito and Prof. Patrick Hopkins for enabling thermal characterization critical for evaluating the results. I also acknowledge all those at the High Temperature Materials Laboratory at Oak Ridge National Laboratory (ORNL) who provided excellent support in characterizing and interpreting the laboratory results. It was a fantastic experience to collaborate with Melanie Kirkham, Shawn Reeves, Hsin Wang, Andrew Payzant, Karren More, Lawrence Allard, and Harry Meyer, and I appreciate the support from Christine Goudy and HTML director Edgar Lara-Curzio.

Many thanks to my fellow colleagues and equipment operators that went out of their way to assist with solving the unique problems encountered in laboratory research, in particular ChenNan Sun, Vikram Iyengar, Keye Sun, Jiguang Li, Yang Shen, Longteng Wang, Christian Rothenbach, Tabitha Apple, B.K. Nayak, Yiliang Bao, Joe Beatrice, Alex Lobo, and Richard White. Lastly, I want to thank my wife for supporting me during the long road of creating a dissertation. 


\section{Table of Contents}

Approval Sheet .................................................................................................................. 2

Abstract....................................................................................................................................................... 3

Acknowledgment ................................................................................................................................. 5

List of Equations ....................................................................................................................................... 10

List of Figures............................................................................................................................................... 11

List of Tables ........................................................................................................................................... 13

List of Symbols and Acronyms ................................................................................................................. 14

Chapter 1： Introduction to Thermoelectrics........................................................................... 17

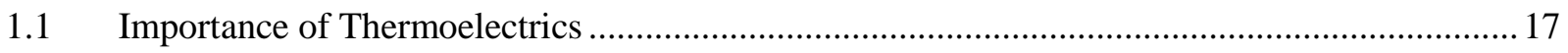

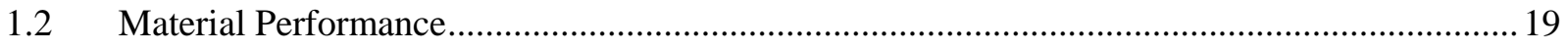

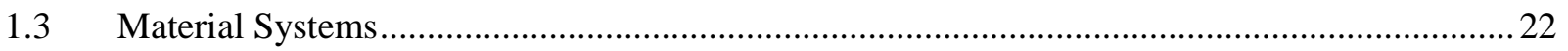

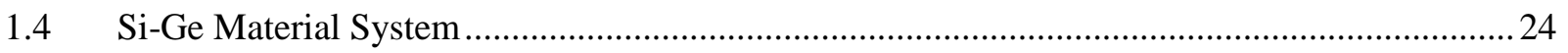

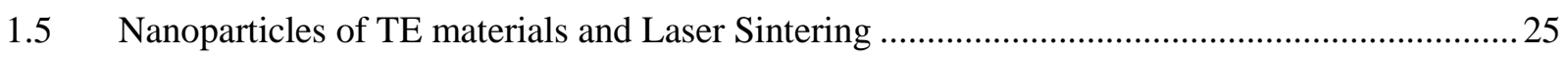

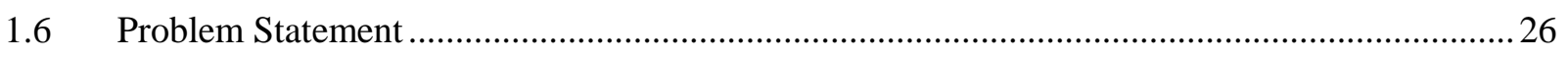

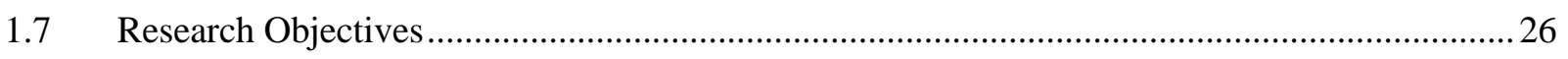

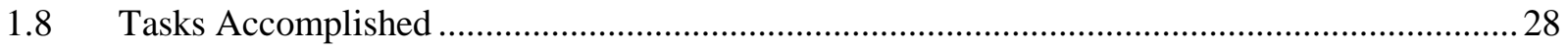

Chapter 2 : Thermoelectric Theory ……........................................................................................ 30

$2.1 \quad$ Concept of Operation ……………………………………………………………….... 30

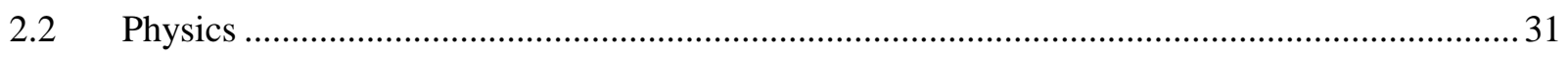

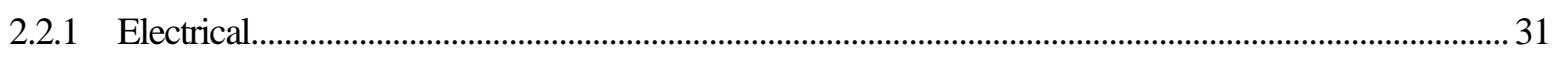

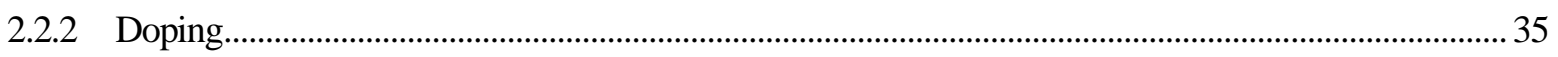

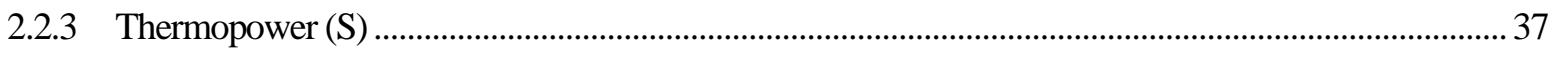

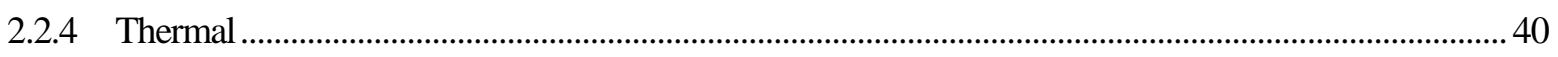

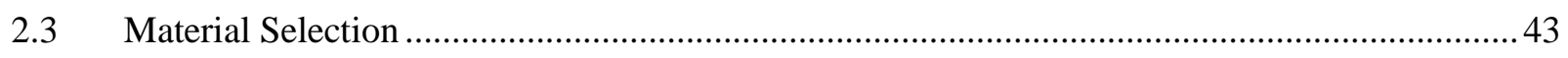

Chapter 3 : Literature Review.................................................................................................... 48

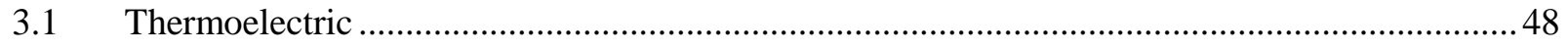

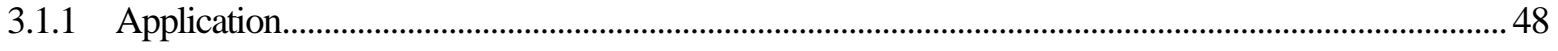

3.1.2 Automotive power generation example ........................................................................................ 51

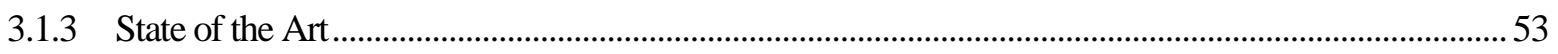

3.1.4 Characterization Techniques ................................................................................................ 58

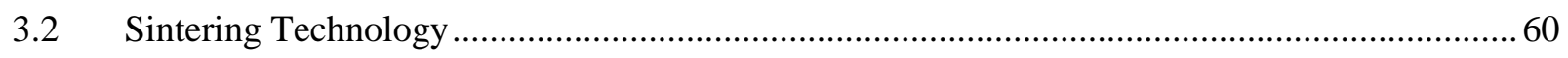




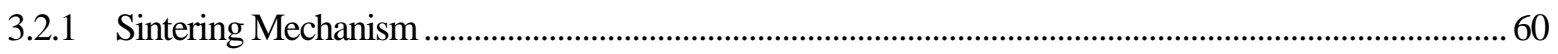

3.2.2 Materials and Constraints ........................................................................................................... 62

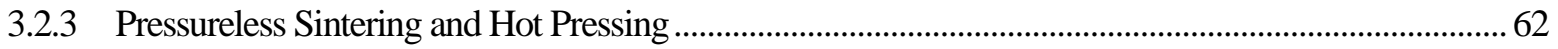

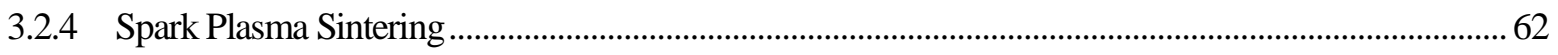

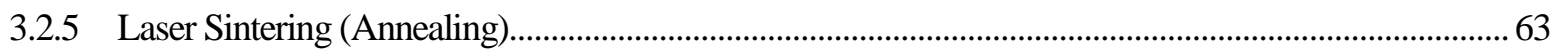

Chapter 4 ： Experimental..............................................................................................................6 65

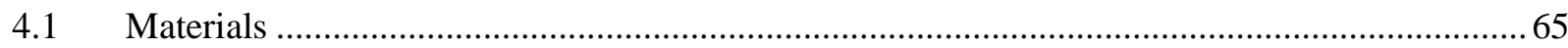

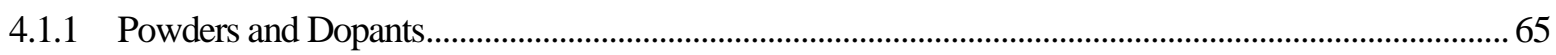

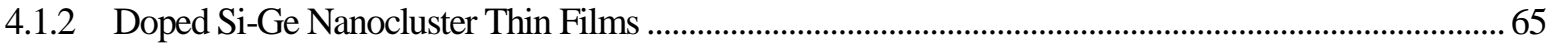

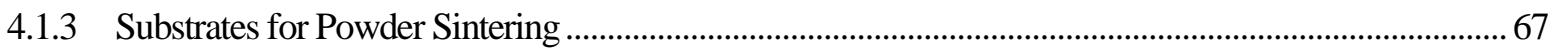

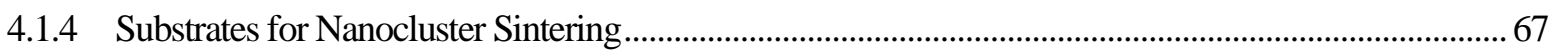

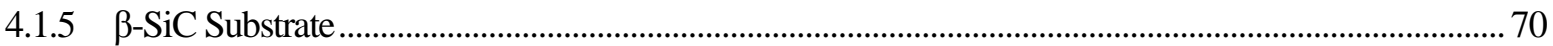

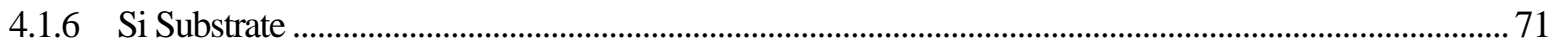

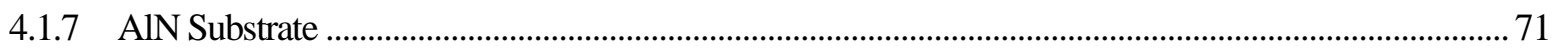

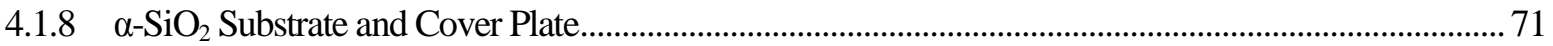

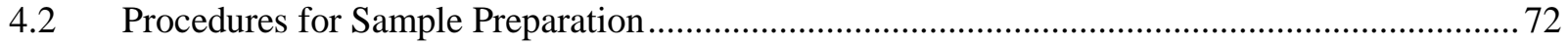

4.2.1 Nanoparticle Processing................................................................................................................. 72

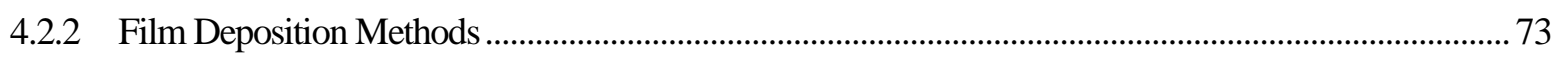

4.2.3 Chamber, Stage, and Induction Heater for use with $940 \mathrm{~nm}$ Laser ....................................................... 74

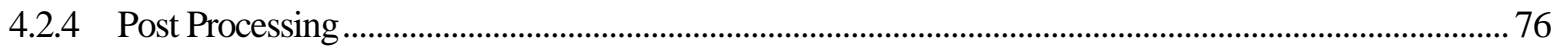

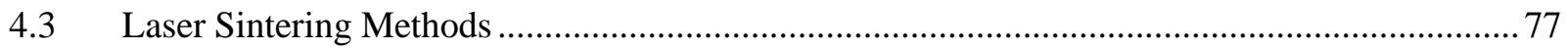

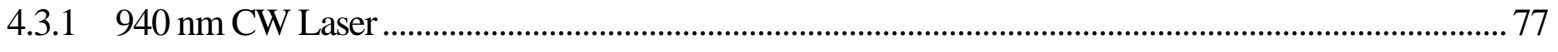

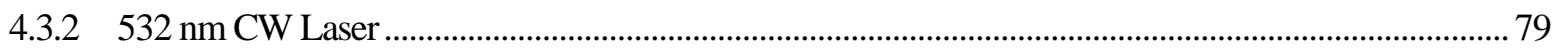

4.3.3 Comparison with Rapid Thermal Anneal (RTA) …………...................................................................... 79

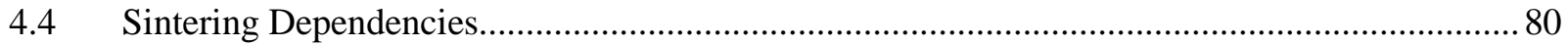

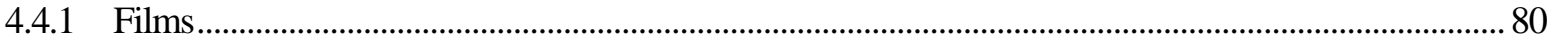

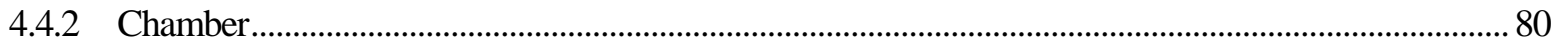

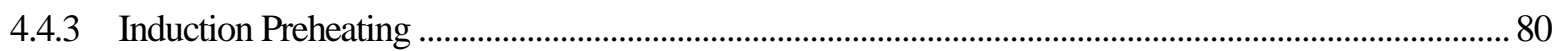

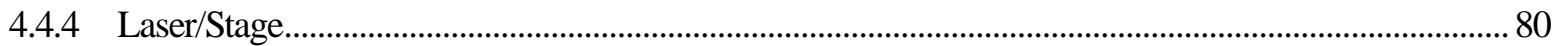

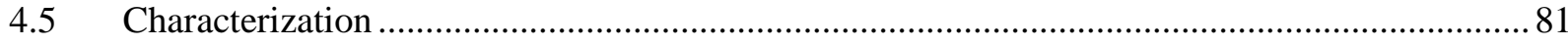

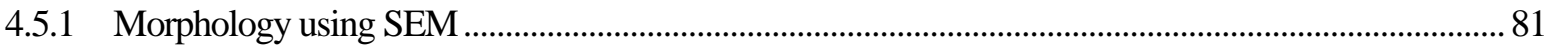

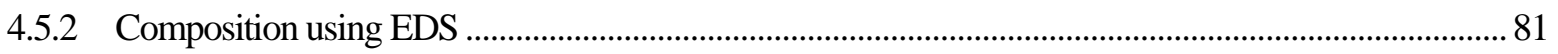

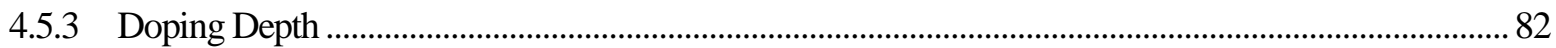

4.5.4 Phases, Structure, and Stability Using X-Ray Diffraction....................................................................... 82

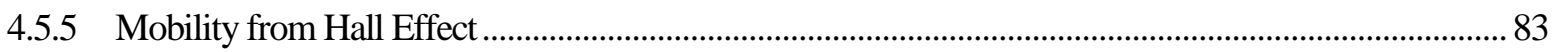

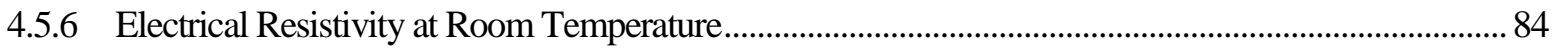

4.5.7 Thermopower and Electrical Conductivity Using Ulvac ZEM-3 vs. Temperature ............................... 84 
4.5.8 Thermal Conductivity using Time-Domain Thermoreflectance vs Temperature .................................. 86

Chapter 5 : $\quad$ Calculation of Si-Ge Material Properties.................................................... 88

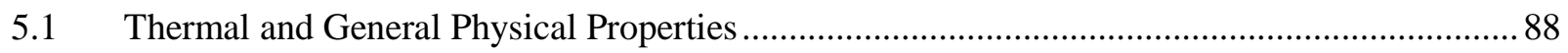

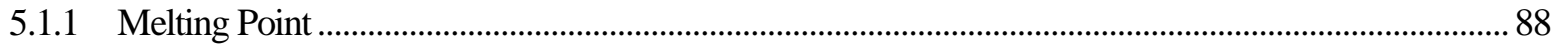

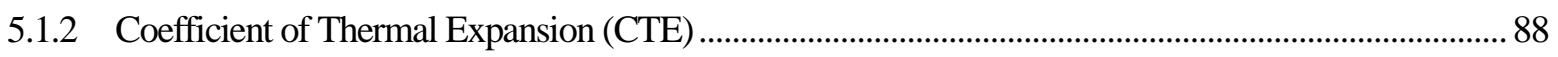

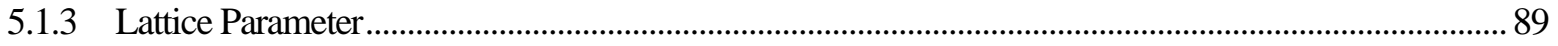

5.1.4 Linear Expansion (Lattice Parameter vs. Temperature) ………................................................................ 90

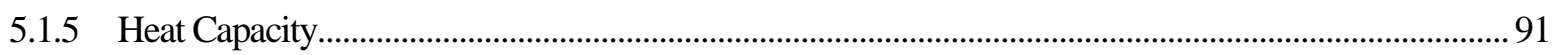

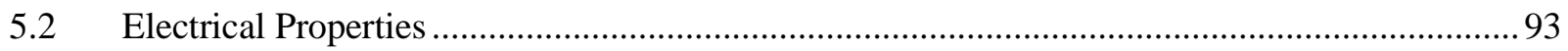

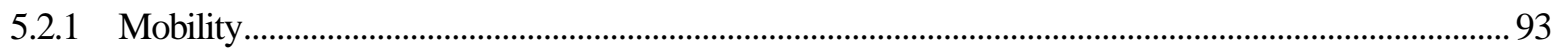

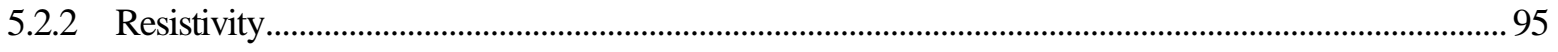

5.2.3 Carrier Scattering Parameter................................................................................................................ 96

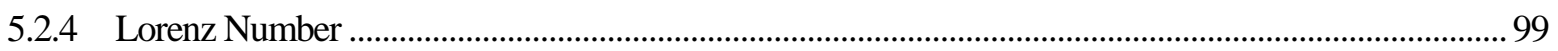

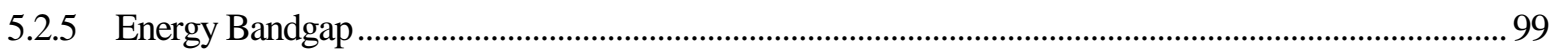

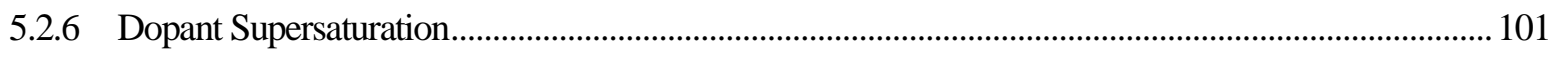

Chapter 6 : Results and Discussions ........................................................................................ 103

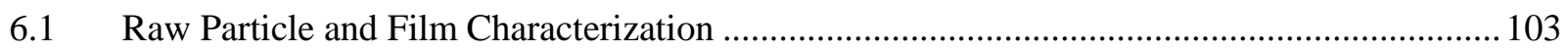

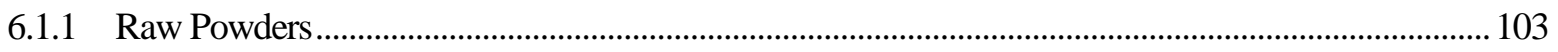

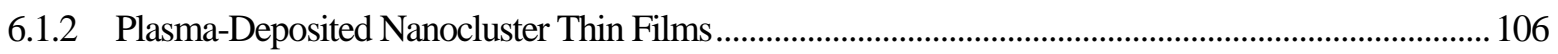

6.1.3 Summary ........................................................................................................................................ 109

$6.2 \quad$ Preheating using the Induction Heater (I.H.) …............................................................. 110

6.3 Sintering Results of Si-Ge Thin Films Using 940 nm CW Laser ......................................... 112

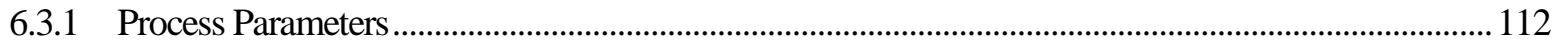

6.3.2 Morphology and Composition .................................................................................................. 116

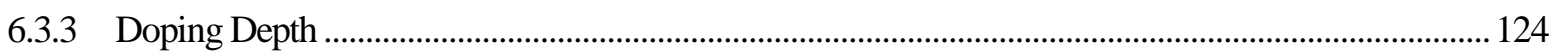

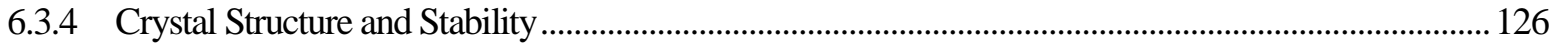

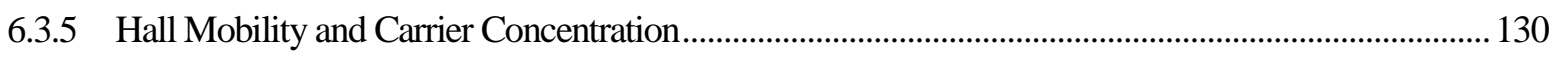

6.3.6 Electrical Resistivity at Room Temperature................................................................................. 132

6.3.7 Electrical Conductivity and Seebeck Coefficient to High Temperature ............................................. 133

6.3.8 Thermal Conductivity to High Temperature ............................................................................................ 139

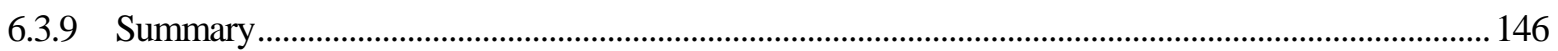

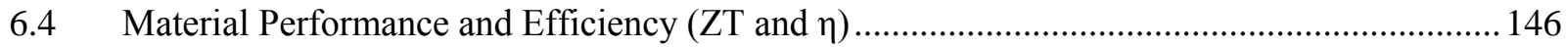

Chapter 7 : Additional Research Related to Thermoelectrics.............................................. 148

7.1 Sintering of Si-Ge Powders Using 940 nm CW Laser........................................................ 148

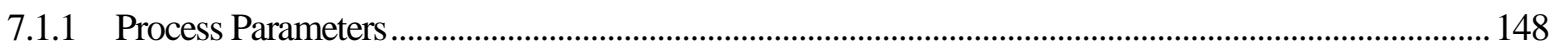

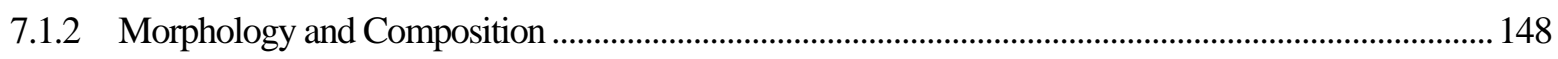

7.1.3 Crystal Structure Characterization ........................................................................................................... 150 


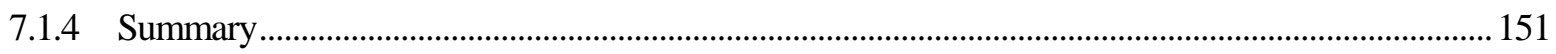

7.2 Sintering of Si-Ge Thin Films Using Rapid Thermal Anneal................................................ 151

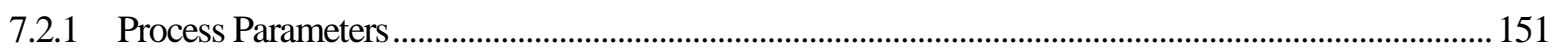

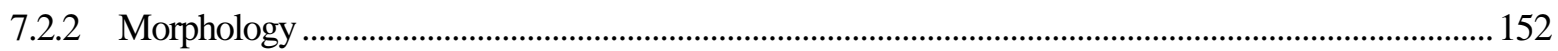

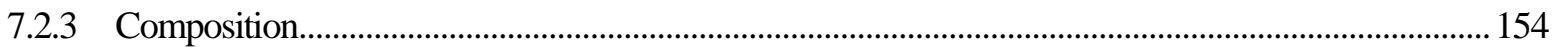

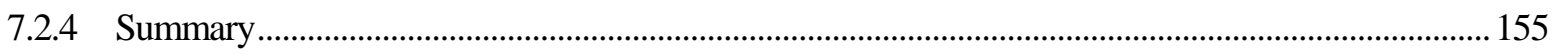

7.3 Sintering of Si-Ge Thin Films Using 532 nm CW Laser.................................................... 155

Chapter 8 : Additional Research Related to Laser Processing of Materials....................... 156

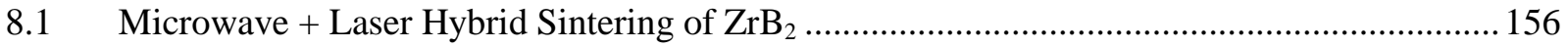

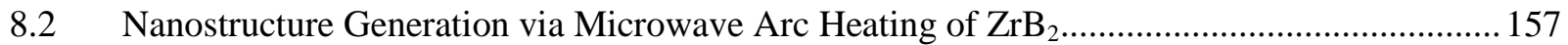

8.3 Laser Cladding of Inconel 690 Superalloy ....................................................................... 158

Chapter 9 : Conclusion and Future Plan............................................................................ 159

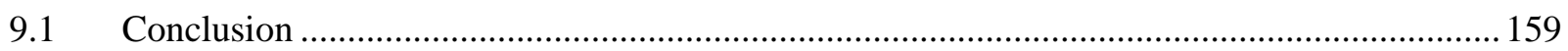

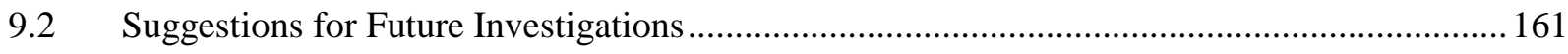

References................................................................................................................................................. 164

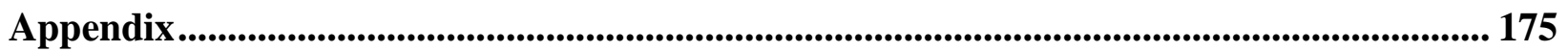

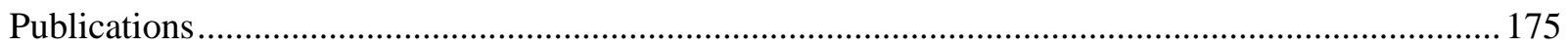

[1] "Thermoelectric films of CW laser sintered doped Si-Ge nanoclusters" [in progress]....................... 175

[2] “CW laser sintering of Si-Ge nanoclusters" [in progress] .............................................................. 175

[3] "Thermal conductivity of laser sintered $\mathrm{Si}_{0.8} \mathrm{Ge}_{0.2}$ nanoclusters from 80 to $490 \mathrm{~K}$ " [in progress] ....... 175

[4] "Plasma deposition of doped SiGe nanocrystal films: alloy formation and high temperature stability"

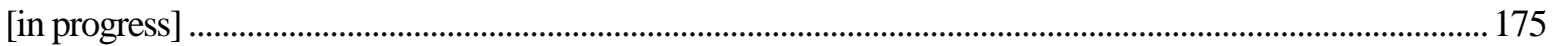

[5] "Inconel 690 laser cladding on Inconel 600 superalloy for corrosion protection in nuclear applications"

[6] "Fabrication of $\mathrm{ZrB}_{2}-\mathrm{Zr}$ cermet using laser sintering technique".................................................... 175

[7] "Nanostructures from Zirconium Diboride and Alumina Ceramics" ................................................ 175

[8] 'Zirconium diboride nanofiber generation via microwave arc heating"'................................................ 175

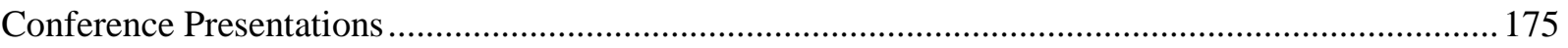

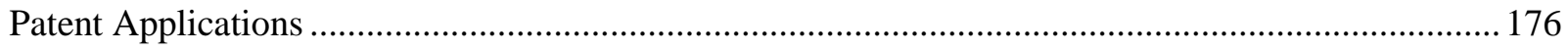




\section{List of Equations}

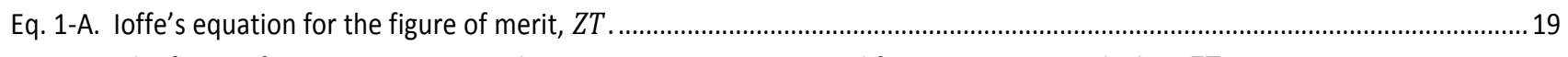

Eq. 1-B. The figure of merit ZT as measured at temperature $T$; integrated from $T_{C}$ to $T_{H}$, to calculate $Z T$.....................................20

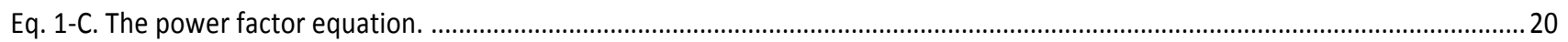

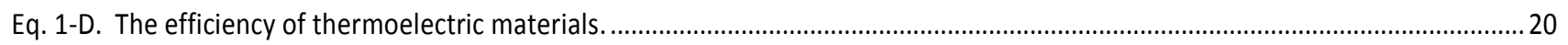

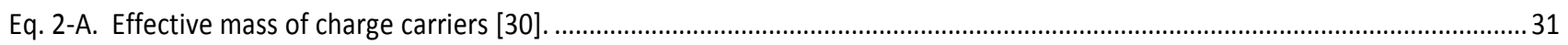

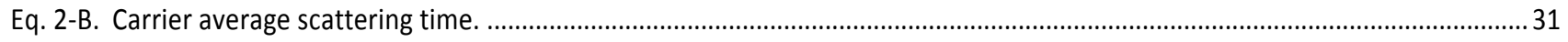

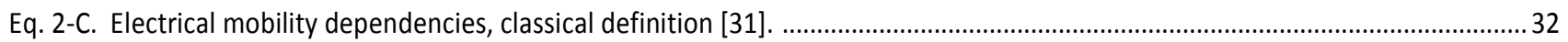

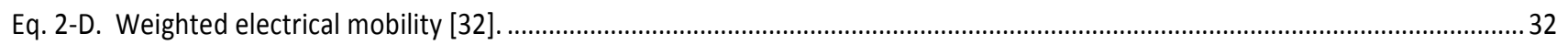

Eq. 2-E. Calculation of the effective carrier density of states function ( $N_{c}$ : electron density, $N_{v}$ : hole density)....................................33

Eq. 2-F. Calculation of carrier concentration from the reduced Fermi energy [34]. ......................................................................33

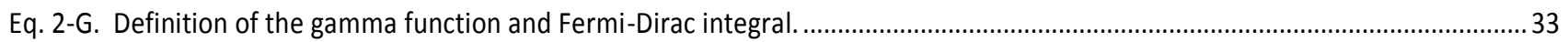

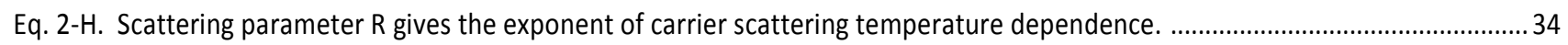

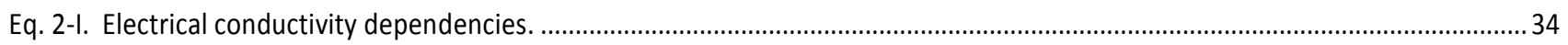

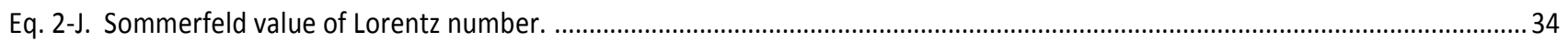

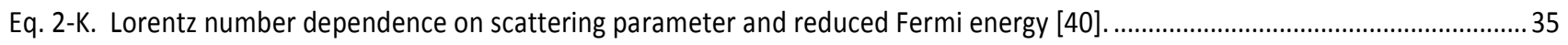

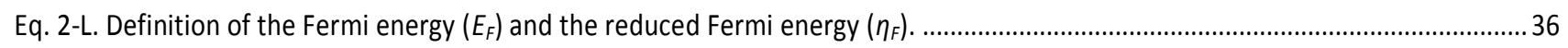

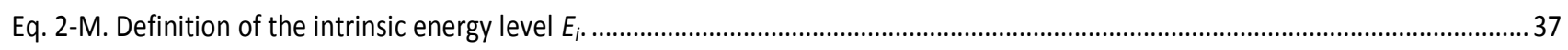

Eq. 2-N. Calculation of the reduced Fermi energy $\left(\eta_{F}\right)$ using Nilsson's approximation [44] ............................................................37

Eq. 2-0. Seebeck coefficient dependence on Fermi energy for nearly free electrons [45]. ...............................................................38

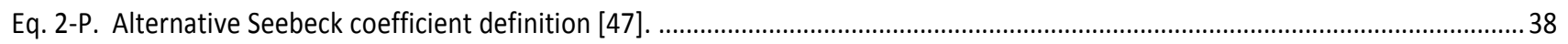

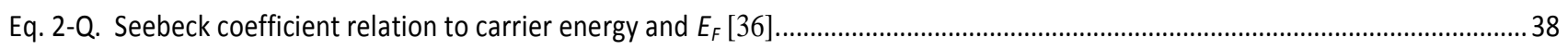

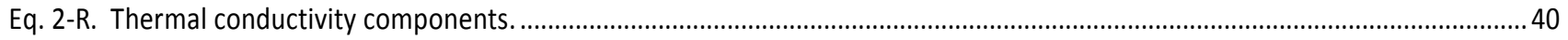

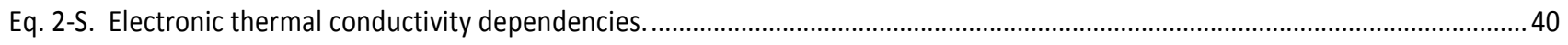

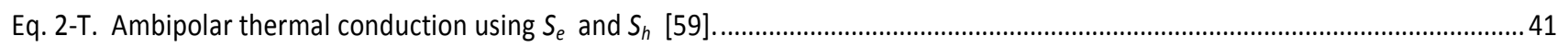

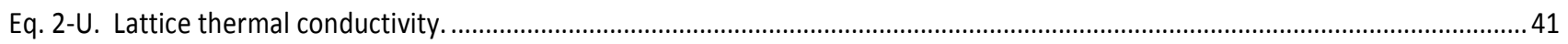

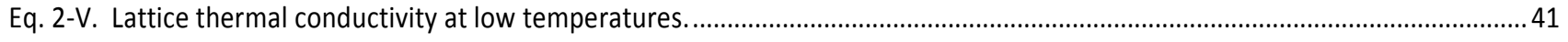

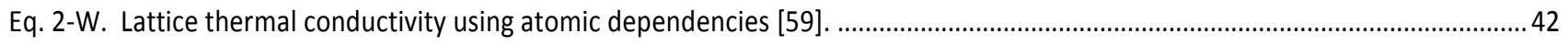

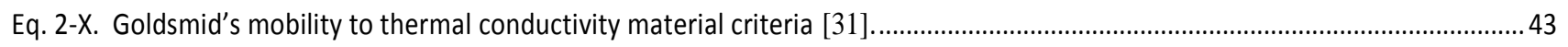

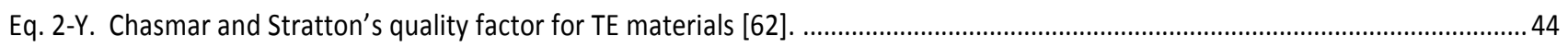

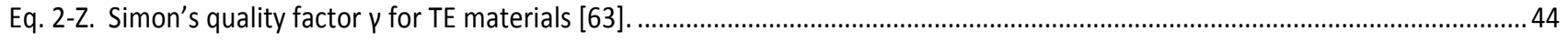

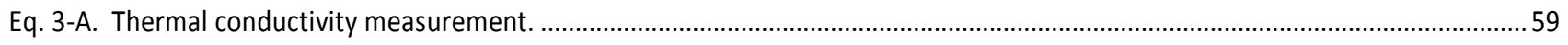

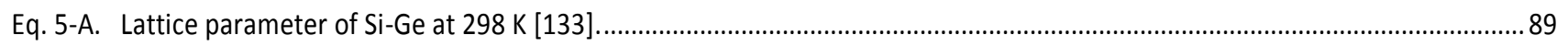

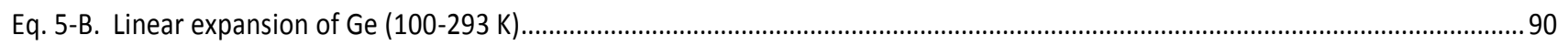

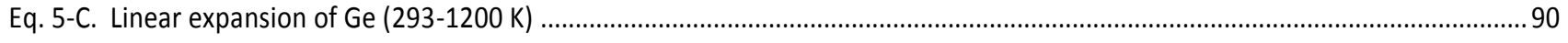

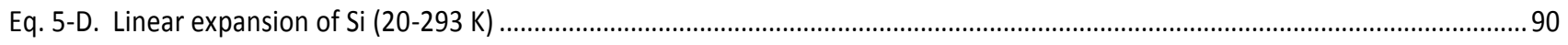

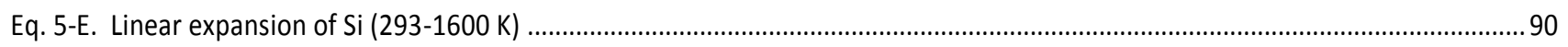

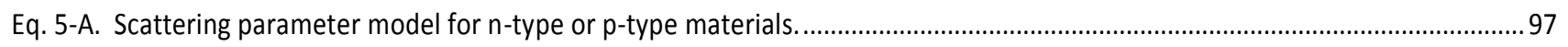




\section{List of Figures}

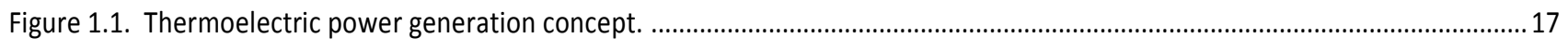

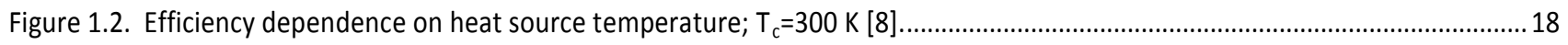

Figure 1.3. TE unicouple with two segments for NASA RTG [13] ............................................................................................19

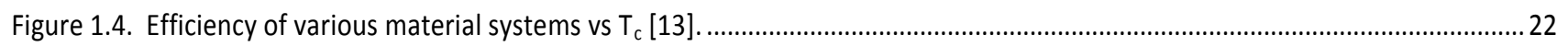

Figure 1.5. ZT of various TE materials, each having an optimum temperature range [19]..............................................................23

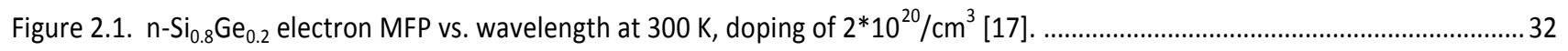

Figure 2.2. ZT dependence of Si-Ge on ionized carrier density at various temperatures [42] . ..........................................................36

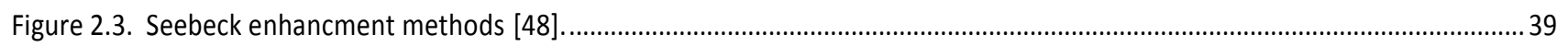

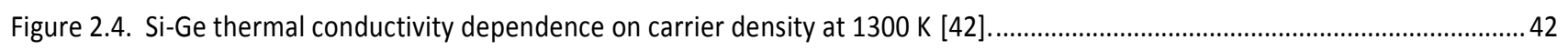

Figure 2.5. $\mathrm{Si}_{0.5} \mathrm{Ge}_{0.5}$ thermal conductivity accumulation vs. phonon MFP [68] ............................................................................ 46

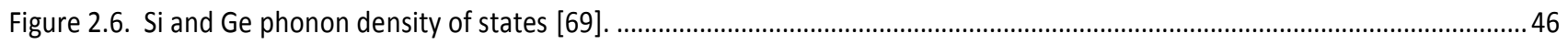

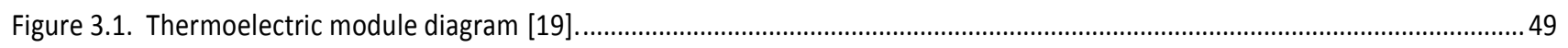

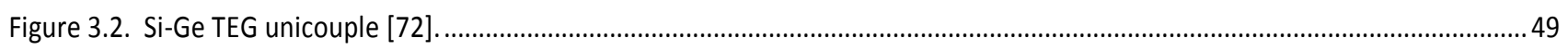

Figure 3.3. TEG from Amerigon: $500 \mathrm{~W}$ from $40 \mathrm{~g} / \mathrm{s}-600^{\circ} \mathrm{C}$ exhaust gas flow; $23 \mathrm{lbs}$ [74] ...................................................5

Figure 3.4. Performance of state of the art thermoelectric materials, with improvements due to nanostructuring [9], [38], [81] ..... 55

Figure 3.5. Lattice thermal conductivity of various TE material systems vs. temperature [19].........................................................56

Figure 3.6. Best Si-Ge figure of merit achieved as of 2012: $\mathrm{n}$ [78], $\mathrm{p}$ [77]....................................................................................5

Figure 3.7. Improved electrical conductivity and power factor via modulation doping [84]...........................................................58

Figure 3.8. Sintering stages of Si-Ge nanoparticles from SEM: a) necking, b) densification, c) percolation .......................................61

Figure 3.9. Morphology of laser sintered Si by Lechner et al. [94] and Si-Ge by Stoib et al. [107] .....................................................64

Figure 4.1. Schematic of nanocluster synthesis developed by Prof. Kortshagen's group at UMN [48] ..............................................66

Figure 4.2. Contact angle of molten $\mathrm{Si}\left(\theta^{\circ}\right)$ vs. bandgap energy of ceramic substrates $(\mathrm{eV})$ [115] ...............................................69

Figure 4.3. CoorsTek HR $\beta$-SiC thermal conductivity vs. temperature......................................................................................... 71

Figure 4.4. $\mathrm{Si}_{0.8} \mathrm{Ge}_{0.2}$ nanocluster thin-film after BOE etch and induction heating. .........................................................................73

Figure 4.5. Chamber design for laser sintering (left: top and right: side)....................................................................................75

Figure 4.6. Chamber: Hybrid heating on Si-Ge on Mo substrate. .........................................................................................

Figure 4.7. Schematic of experimental setup for $940 \mathrm{~nm}$ laser hybrid heating. ...........................................................................78

Figure 4.8. $940 \mathrm{~nm}$ laser output power vs. input current.

Figure 4.9. Hall mobility and carrier concentration measurement setup in Ecopia HMS-3000. ........................................................... 83

Figure 4.10. Hall effect contact geometry for the van der Pauw method.....................................................................................8 84

Figure 4.11. Ulvac ZEM-3 sample mounting image and schematic from manual...........................................................................8 85

Figure 4.12. Model of Si-Ge thin film on silica substrate with Ag contacts, ready for Ulvac ZEM...................................................... 86

Figure 5.1. Coefficients of thermal expansion for $\mathrm{Si}$, Ge and $\beta$-SiC. ............................................................................................. 89

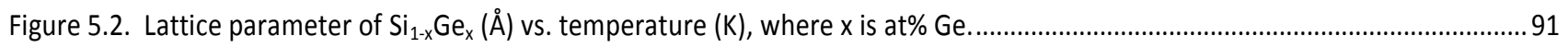

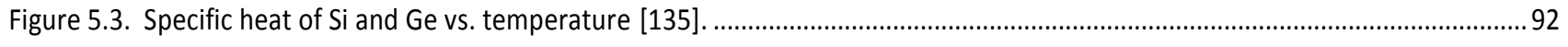

Figure 5.4. Si-Ge hall mobility dependence on carrier density at various temperatures [42] ............................................................94

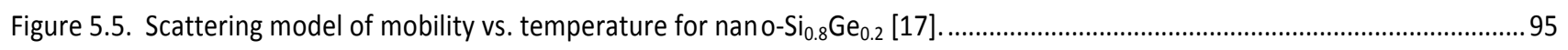

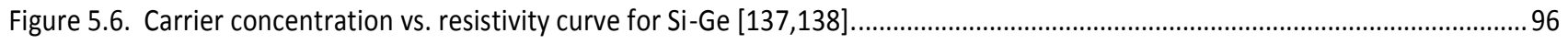

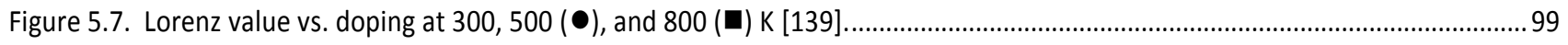

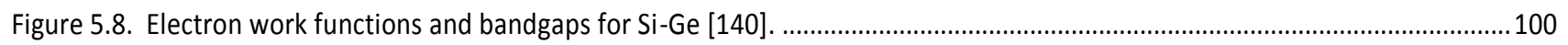

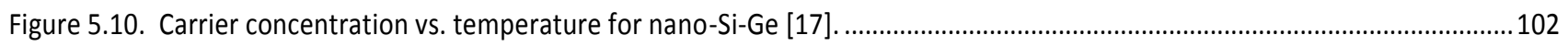

Figure 6.1. Silicon nanopowder image from SEM. ............................................................................................................ 103 


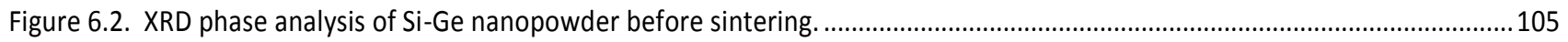

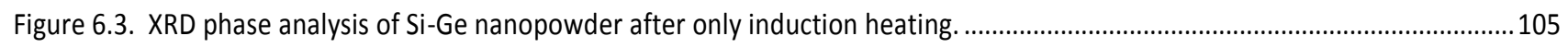

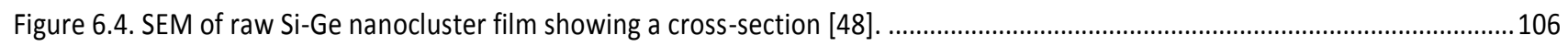

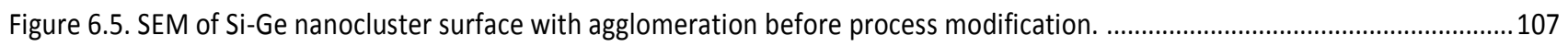

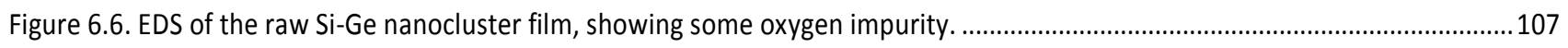

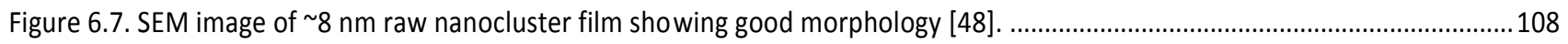

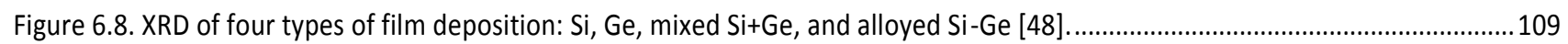

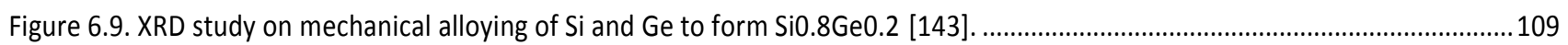

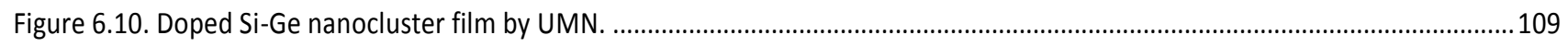

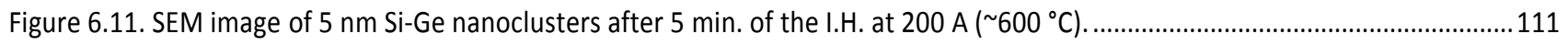

Figure 6.12. SEM image of Si-Ge nanocluster sintered film after laser-induced ignition.....................................................................111

Figure 6.13. SEM images of induction heated Si-Ge nanoclusters showing surface effects................................................................112

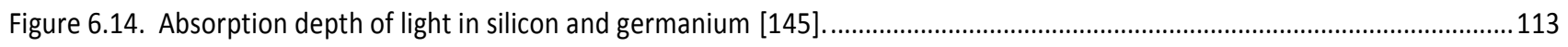

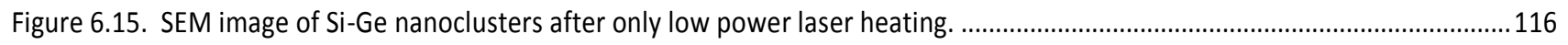

Figure 6.16. Visible image of laser sintered nanocluster film showing high reflectivity. ..................................................................117

Figure 6.17. Visible image of sintered nanocluster film on silica comparing scan line overlap. ........................................................117

Figure 6.18. Optical image of laser sintered nanocluster film showing wetting................................................................................118

Figure 6.19. Optical image of laser sintered nanocluster film showing percolation and balling........................................................119

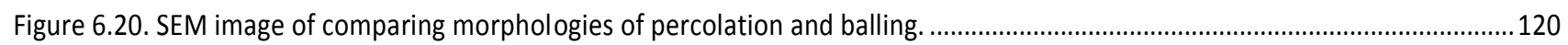

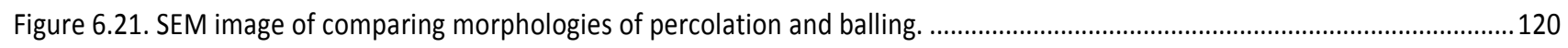

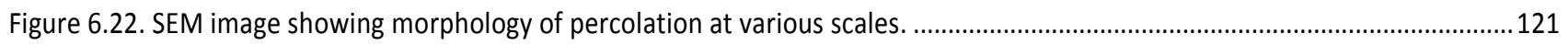

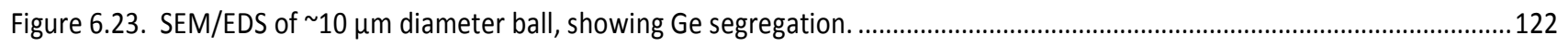

Figure 6.24. SEM of laser sintered nanocluster film showing densification and continuity. ...............................................................123

Figure 6.26. SEM of laser sintered nanocluster film on SiC showing densification and adhesion. .....................................................124

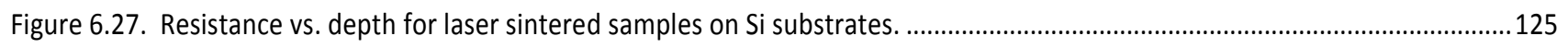

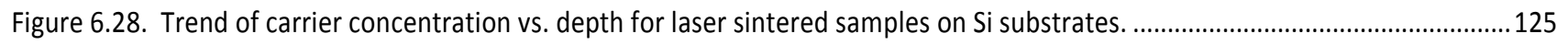

Figure 6.29. Si-Ge $5 \mathrm{~nm}$ nanocrystal phase stability by HT-XRD after only induction heating...........................................................127

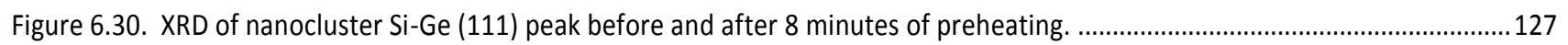

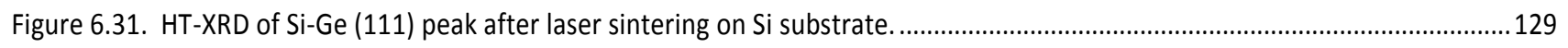

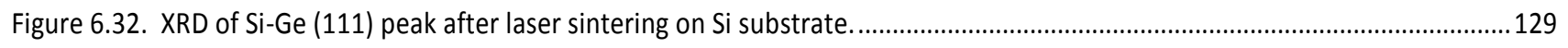

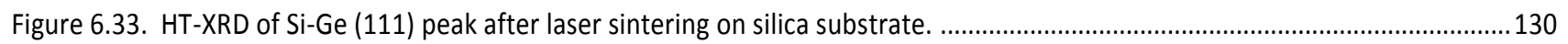

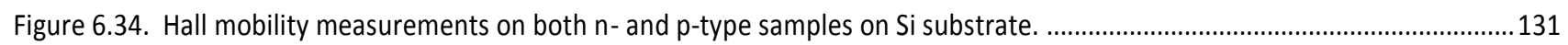

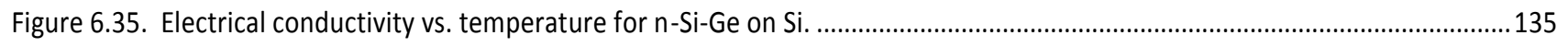

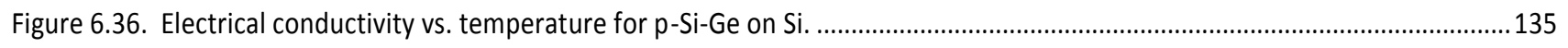

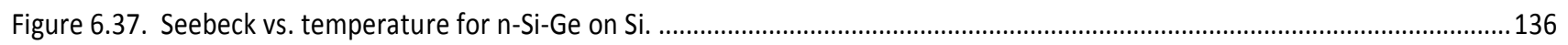

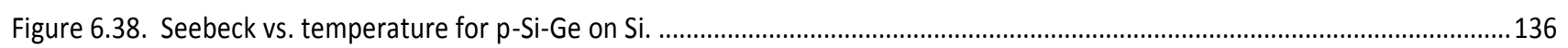

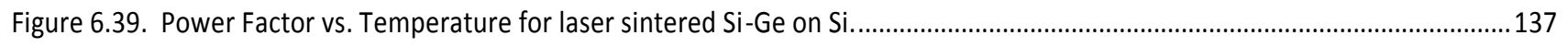

Figure 6.40. p-Si-Ge thin-film thermopower and resistivity after laser sintering. ..............................................................................138

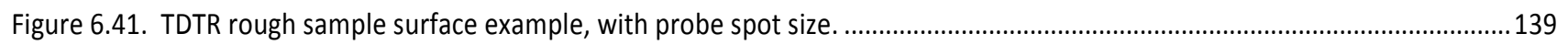

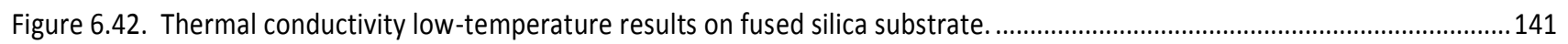

Figure 6.43. Thermal conductivity low-temperature comparison to literature.................................................................................142

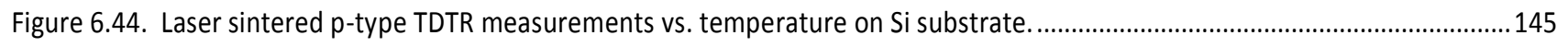

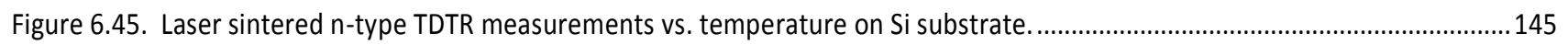

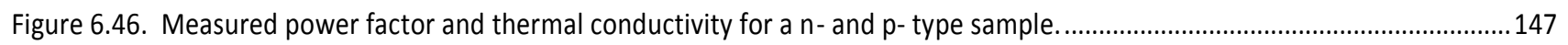

Figure 6.47. ZT projection for two laser sintered samples, given Si substrate dilution......................................................................147

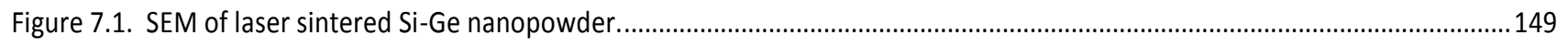


Figure 7.2. Si-Ge nanopowder composition by EDS in SEM after sintering by $940 \mathrm{~nm}$ laser.

Figure 7.3. XRD phase analysis of Si-Ge nanopowder after laser sintering on Mo substrate. ..........................................................150

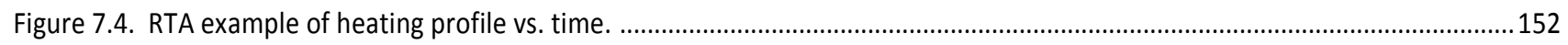

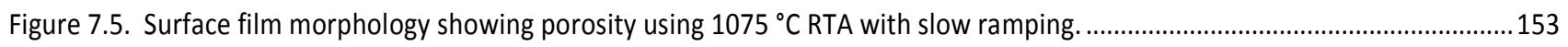

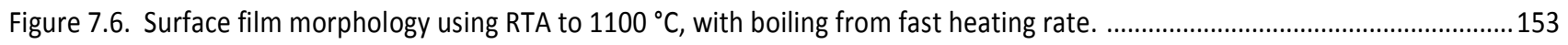

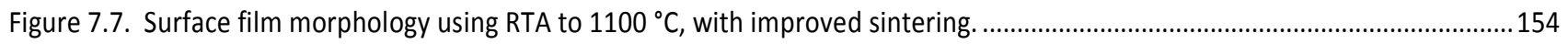

Figure 7.8. Composition when using RTA, exhibiting nitrogen contamination from the chamber. ..................................................154

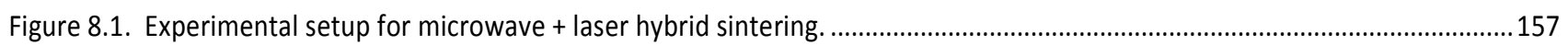

\section{List of Tables}

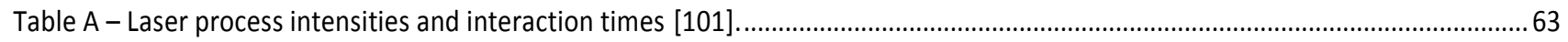

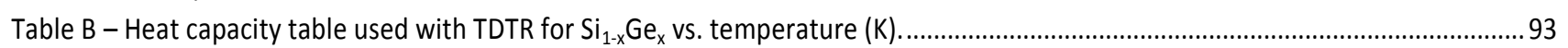

Table C - Scattering parameter estimation for Si-Ge using temperature, doping, and grain size. .......................................................98

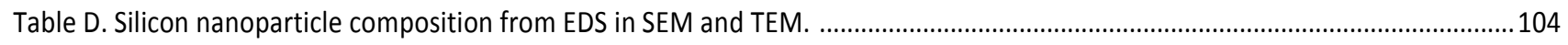

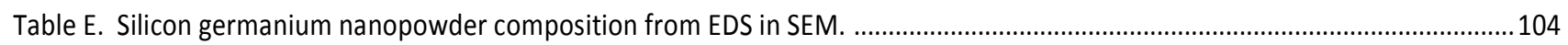

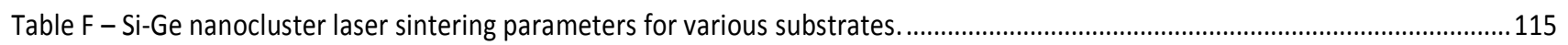

Table G - Si-Ge sintering parameters for samples on silicon characterized to high temperature. ......................................................115

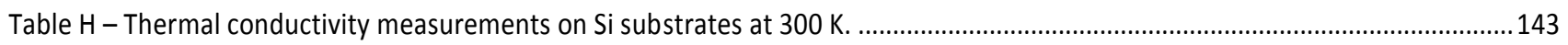




\section{List of Symbols and Acronyms}

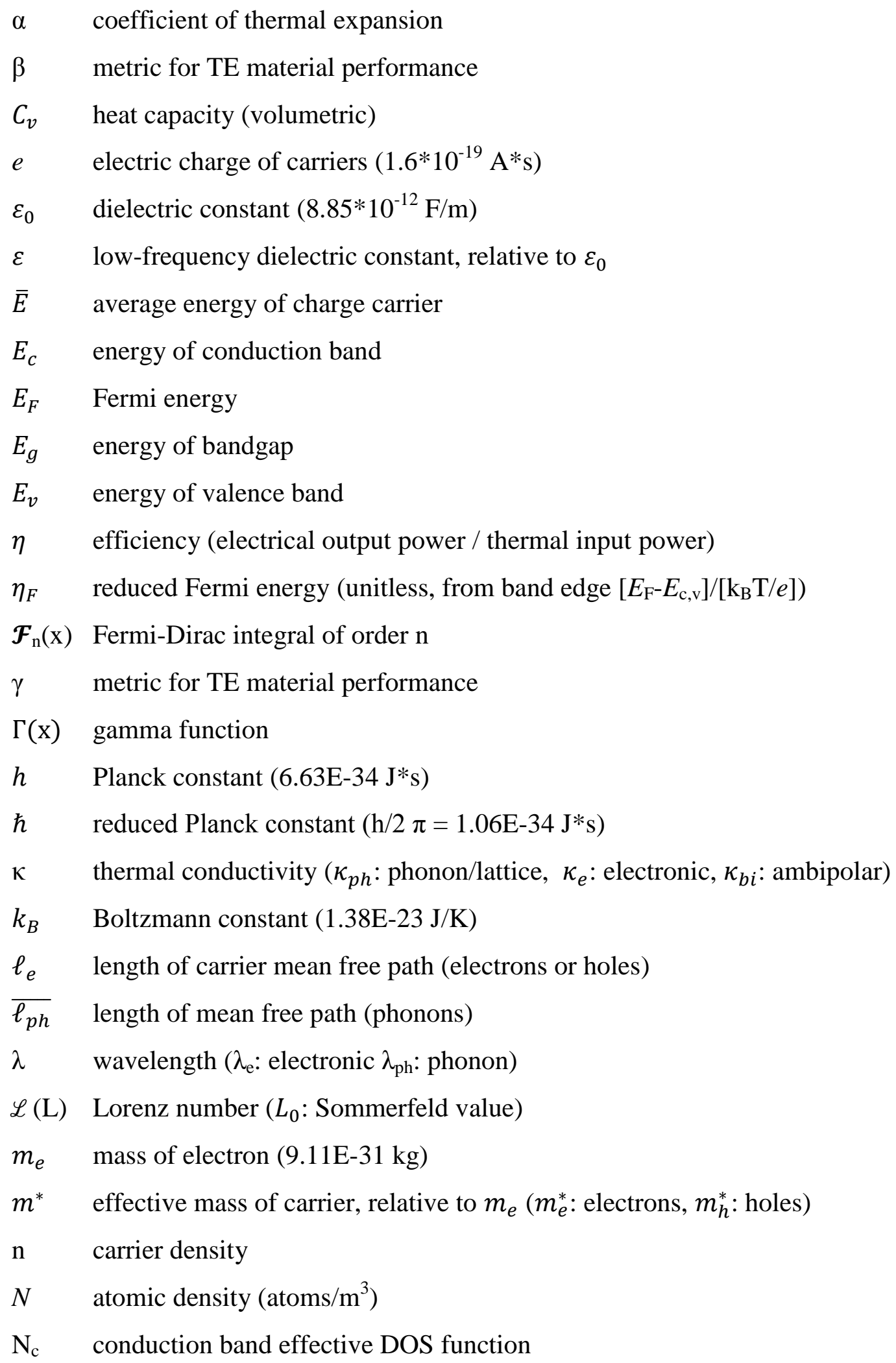




\begin{tabular}{|c|c|}
\hline \multicolumn{2}{|c|}{ valence band effective DOS function } \\
\hline $\mathrm{pi}$ & 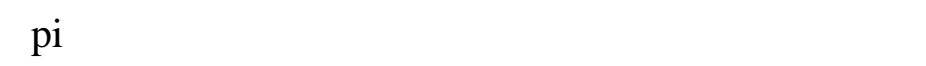 \\
\hline$\Omega$ & ohms \\
\hline el & electrical resistivity \\
\hline $\mathrm{sc}$ & scattering parameter, here $\mathrm{R}=\mathrm{s}+1 / 2$ \\
\hline $\mathrm{Se}$ & Seebeck coefficient (thermopower) \\
\hline $\mathrm{sc}$ & scattering parameter ( $\mathrm{s}_{\mathrm{e}}$ : electrons $\mathrm{s}_{\mathrm{h}}$ : holes) \\
\hline elc & electrical conductivity $\left(\sigma_{\mathrm{e}}:\right.$ electrons $\sigma_{\mathrm{h}}$ : holes) \\
\hline re & relaxation time \\
\hline $\mathrm{T}$ & temperature \\
\hline av & average temperature \\
\hline $\mathrm{D}$ & Debye temperature \\
\hline ca & carrier mobility ( $\mu_{\mathrm{e}}:$ electrons $\mu_{\mathrm{h}}$ : holes) \\
\hline ve & velocity of sound ( $v_{D}:$ Debye $)$ \\
\hline at $\%$ & atomic percentage \\
\hline $\mathrm{wt} \%$ & weight percentage \\
\hline CTE & Coefficient of Thermal Expansion \\
\hline DOS & Density Of States \\
\hline EDS & Energy Dispersive x-ray Spectroscopy \\
\hline FD & Fermi-Dirac \\
\hline HT-XRD & High Temperature X-Ray Diffraction \\
\hline I.H. & Induction Heater \\
\hline MFP & Mean Free Path \\
\hline nc & nanocrystal \\
\hline $\mathrm{NC}$ & NanoCluster \\
\hline PECVD & Plasma-Enhanced Chemical Vapor Deposition \\
\hline $\mathrm{PF}$ & Power Factor \\
\hline RIE & Reactive Ion Etching \\
\hline RTA & Rapid Thermal Anneal \\
\hline SEM & Scanning Electron Microscope \\
\hline SNR & Signal to Noise Ratio \\
\hline
\end{tabular}


TDTR Time-Domain ThermoReflectance

TE

TEG

TEM

UHTC

XRD
Thermoelectric(s)

Thermoelectric Generator

Transmission Electron Microscope

Ultra High Temperature Ceramic

X-Ray Diffraction 


\section{Chapter 1 : Introduction to Thermoelectrics}

\subsection{Importance of Thermoelectrics}

Thermoelectric (TE) materials convert a temperature gradient to an electrical potential, providing a non-mechanical source of electricity. TE devices have tremendous potential to recover wasted heat generated during energy conversion processes. With waste heat availability estimated at $192 \mathrm{GW}$ in the US [1] and $15 \mathrm{TW}$ worldwide [2], thermoelectrics are an attractive alternative energy source for improving the net efficiency of power generation. This improved efficiency, along with the absence of any operational pollution, reduces greenhouse gas production and reliance on fossil fuels. The importance of clean energy generation has been underscored by the shift in US policy and societal views towards reducing greenhouse gas generation and improving energy security by reducing reliance on foreign energy sources. Significant high grade waste heat is available from gas turbines and industrial processes such as the manufacturing of aluminum, steel, and glass (>100 $\mathrm{MW}_{\mathrm{e}}$ output possible in the US) [3].

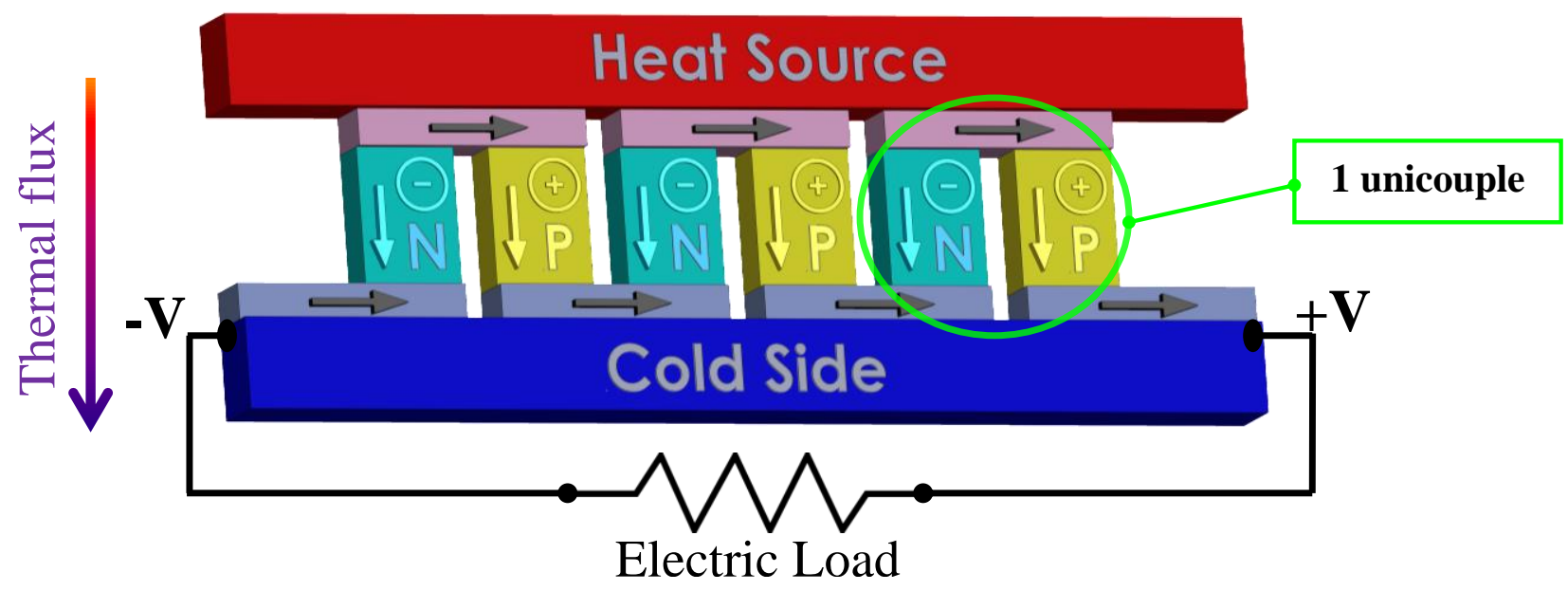

Figure 1.1. Thermoelectric power generation concept.

NASA has a long history of implementing high-temperature thermoelectric generators (TEG's) alongside radioisotope thermoelectric generators (RTG's) to provide electric power on deep space missions where solar power is insufficient. The RTG's provide a stable source of thermal flux across the TE unicouple elements, which then generate a voltage and drive a current across the load, as in Figure 1.1. The TE module in this figure is made from three unicouples, where a unicouple consists of one $n$-element and one p-element. On these space craft long-term 
TEG reliability has been proven, with over $10^{12}$ device-hours and not a single unicouple failure [4]. NASA still drives research for improved TEG performance for applications, such as the Mars rovers, where efficiency and weight are equally important [3]. Forthcoming applications may integrate with automotive and turbine engines of nearly any fuel source. There have even been studies comparing the suitability of thermoelectric vs. photovoltaic power generation $[5,6]$.

Successful adoption of thermoelectrics requires improved performance as documented by numerous researchers such as Bux et al. [7]. The efficiency is dependent upon the material figure of merit (ZT), the temperature differential across the device, and the heat source temperature as shown in Figure 1.2, where $T_{c}$ is the cold side temperature.

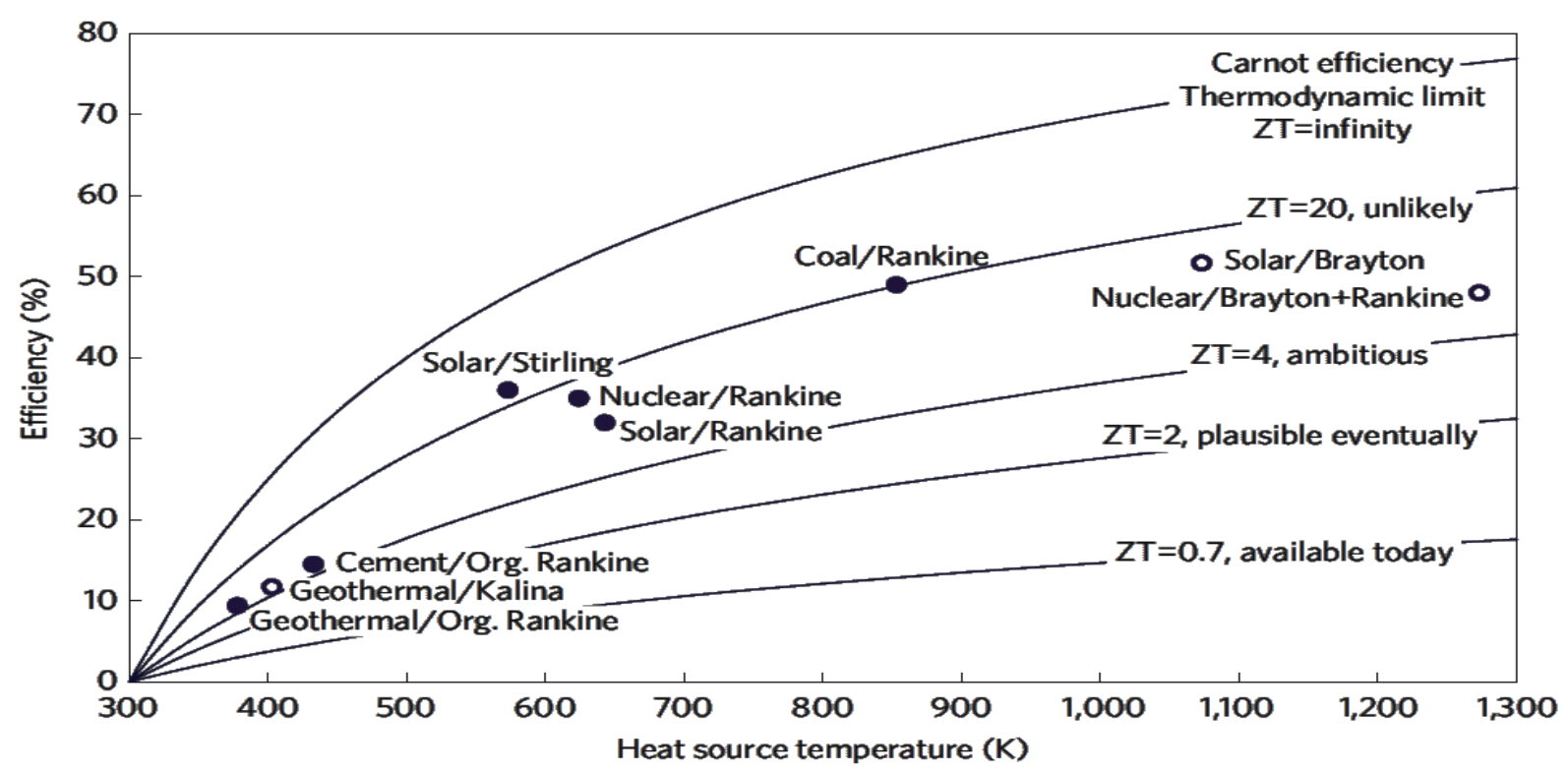

Figure 1.2. Efficiency dependence on heat source temperature; $\mathrm{T}_{\mathrm{c}}=300 \mathrm{~K}$ [8].

Significant progress has been made in thermoelectric power conversion devices, with demonstrated element efficiencies exceeding $8 \%$ [9], and potential segmented efficiencies exceeding 10\% [3,10]. Jet Propulsion Laboratory has even recently demonstrated segmented efficiencies $>15 \%$ operating from 373 to $1273 \mathrm{~K}$ [3]. Segmentation is defined as connecting multiple TE elements of different material systems in series both electrically and thermally, which allows running each material in the optimum temperature range [11], as illustrated in Figure 1.3. Although demonstration efficiencies have been promising, the commercial potential of thermoelectric devices can be realized only by further improvements in efficiency, long term stability after many thermal cycles to high temperatures, and lower cost of fabrication. A 
worldwide research effort is being carried out to accomplish these improvements. DOE lists the efficiency goal as $>20 \%$ [12], while NASA's goal is $>15 \%$ but also with specific power $>10$ $\mathrm{W}_{\mathrm{e}} / \mathrm{kg}[3]$.

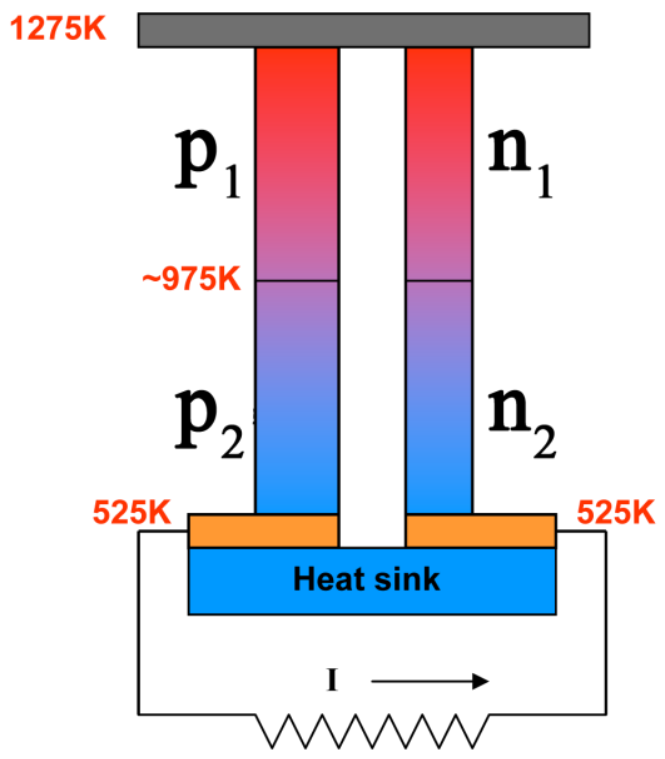

Figure 1.3. TE unicouple with two segments for NASA RTG [13].

\subsection{Material Performance}

Thermoelectric performance is defined in terms of the dimensionless figure of merit (“ZT") of a material system, as proposed by Ioffe in 1957 [14] in Eq. 1-A.

$$
\begin{gathered}
Z \bar{T}=\frac{(\sigma: \text { electrical conductivity }) *(S: \text { Seebeck coefficient })^{2}}{\left(\kappa: \text { thermal conductivity }=\kappa_{e}: \text { electron }+\kappa_{p h}: \text { phonon }\right)} *(\bar{T}: \text { average temperature }) \\
\bar{T}=\frac{T_{H}+T_{C}}{2} ; \begin{array}{l}
T_{H}: \text { Hot }- \text { side temperature }(\mathrm{K}) \\
T_{C}: \text { Cold }- \text { side temperature }(\mathrm{K})
\end{array}
\end{gathered}
$$

Eq. 1-A. Ioffe's equation for the figure of merit, $Z \bar{T}$.

The figure of merit can be specified as either $Z T$ or $Z \bar{T}$, with $Z T$ often being the peak value at a specific measured temperature (used in research) and $Z \bar{T}$ being the average across a specified temperature range (often used for applications). ZT equals $Z \bar{T}$ when $T=\bar{T}$, which occurs when $T_{H} \approx T_{C}$. All three parameters $(\sigma, \kappa$, and $S)$ are temperature dependent and thus to accurately calculate $Z \bar{T}$ all three variables must be known as a function of temperature and the resulting data integrated over a specified temperature range, as given by the integral in Eq. 1-B. 


$$
\begin{gathered}
Z T=\frac{\sigma(T) * S(T)^{2}}{\kappa(T)} *(T), \text { which integrating } T \text { from } T_{C} \text { to } T_{H} \text { gives: } Z \bar{T}=\int_{T_{C}}^{T_{H}} \frac{\sigma(T) * S(T)^{2}}{\kappa(T)} *(T) \\
\begin{array}{c}
\sigma: \text { electrical conductivity }\left(\Omega^{-1} \mathrm{~cm}^{-1}\right), S: \text { Seebeck coefficient }(\mathrm{V} / \mathrm{K}) . \\
\kappa: \text { thermal conductivity }(\mathrm{W} / \mathrm{m} / \mathrm{K}), T: \text { temperature }(\mathrm{K}), \\
\kappa=\kappa_{e}+\kappa_{p h} \text {, where: } \kappa_{e}: \text { electronic, } \kappa_{p h}: \text { phonon/lattice }
\end{array}
\end{gathered}
$$

Eq. 1-B. The figure of merit ZT as measured at temperature $T$; integrated from $T_{C}$ to $T_{H}$, to calculate $Z \bar{T}$.

$$
\text { Power Factor }(P F)=\sigma * S^{2}
$$

Eq. 1-C. The power factor equation.

Since $\sigma$ and $S$ are inversely related, material performance cannot be accurately evaluated by these parameters individually. Rather, the Power Factor (PF) is used to evaluate the electrical performance of materials, as in Eq. 1-C.

$$
\operatorname{Efficiency}(\eta)=\frac{\text { Electrical Power }}{\text { Thermal Power }}=\frac{T_{H}-T_{C}}{T_{H}} * \frac{\sqrt{1+Z \bar{T}}-1}{\sqrt{1+Z \bar{T}}+\frac{T_{C}}{T_{H}}}
$$

Eq. 1-D. The efficiency of thermoelectric materials.

The primary goal in this field is to improve thermal to electric conversion to ZT > 2 [15]. Achieving an element efficiency of $>15 \%$ would facilitate market penetration [2], as thermoelectrics would then be cost competitive with current power sources. In order to realize a net ZT improvement, the thermal conductivity must be minimized without significantly reducing the electrical conductivity or the thermopower (i.e., Seebeck coefficient). To attain good electrical conductivity, semiconductors like silicon and germanium must be doped, typically to a degenerate level for thermoelectrics. Doping of thermoelectric semiconductors involves balancing the material properties since all three factors $(\sigma, S, \kappa)$ are dependent upon carrier density. Excessive doping provides improved electrical conductivity but sacrifices a loss of thermopower and gain in thermal conductivity. As shown in the power factor equation, ZT is proportional to the thermopower squared, thus it is critical to retain a high thermopower.

The ideal combination of high 1) electrical conductivity, 2) thermopower, and 3) thermal resistivity is known as a Phonon-Glass Electron-Crystal (PGEC) [16]. An ideal PGEC performs 
electrically like a single crystal, but thermally like an amorphous solid. Given the doping constraints to achieve the largest power factor, the most promising method for approaching a PGEC for a given TE material is to reduce the thermal conductivity by nanostructuring to minimize phonon heat transfer. Nanostructuring of TE materials achieves the desired reduction in lattice thermal conductivity through enhanced phonon scattering [7]. Nano-sized grains possess a high density of interfaces, greatly enhancing the probability of phonon scattering, therefore inhibiting thermal transfer without excessively hindering carrier mobility [7]. At room temperature, the charge carriers have a much shorter mean free path than phonons, on the order of a couple nanometers versus $\sim 100 \mathrm{~nm}$ [17], thus the nanograins preferentially filter the longer wavelength phonons, resulting in significant improvements to the figure of merit [7].

While providing the peak ZT value is the typical method of reporting results, element efficiency $(\eta)$ must be considered to evaluate the true performance over the desired operating range. As thermoelectric power generation is also a Carnot heat engine, a larger temperature difference across the material allows for higher efficiencies. For example, to achieve an efficiency of $25 \%$, system A) with $\mathrm{T}_{\text {cold }}=350 \mathrm{~K}$ and $\Delta \mathrm{T}=800 \mathrm{~K}$ needs a material performance of ZT $=2$, however a lower-temperature system $\mathrm{B}$ ) operating with the same $\mathrm{T}_{\text {cold }}=350 \mathrm{~K}$ but $\Delta \mathrm{T}$ $=400 \mathrm{~K}$ must have $\mathrm{ZT}=4.27$; the smaller temperature difference and lower $\mathrm{T}_{\text {hot }}$ require the material figure of merit to be improved by more than a factor of 2 . Therefore, the system performance is heavily dependent not only on the material performance, but also the application temperature constraints. Figure 1.4 graphically displays the efficiencies of various material systems vs. the cold side temperature. The two points provided are from systems produced by NASA and represent the best state of practice. 


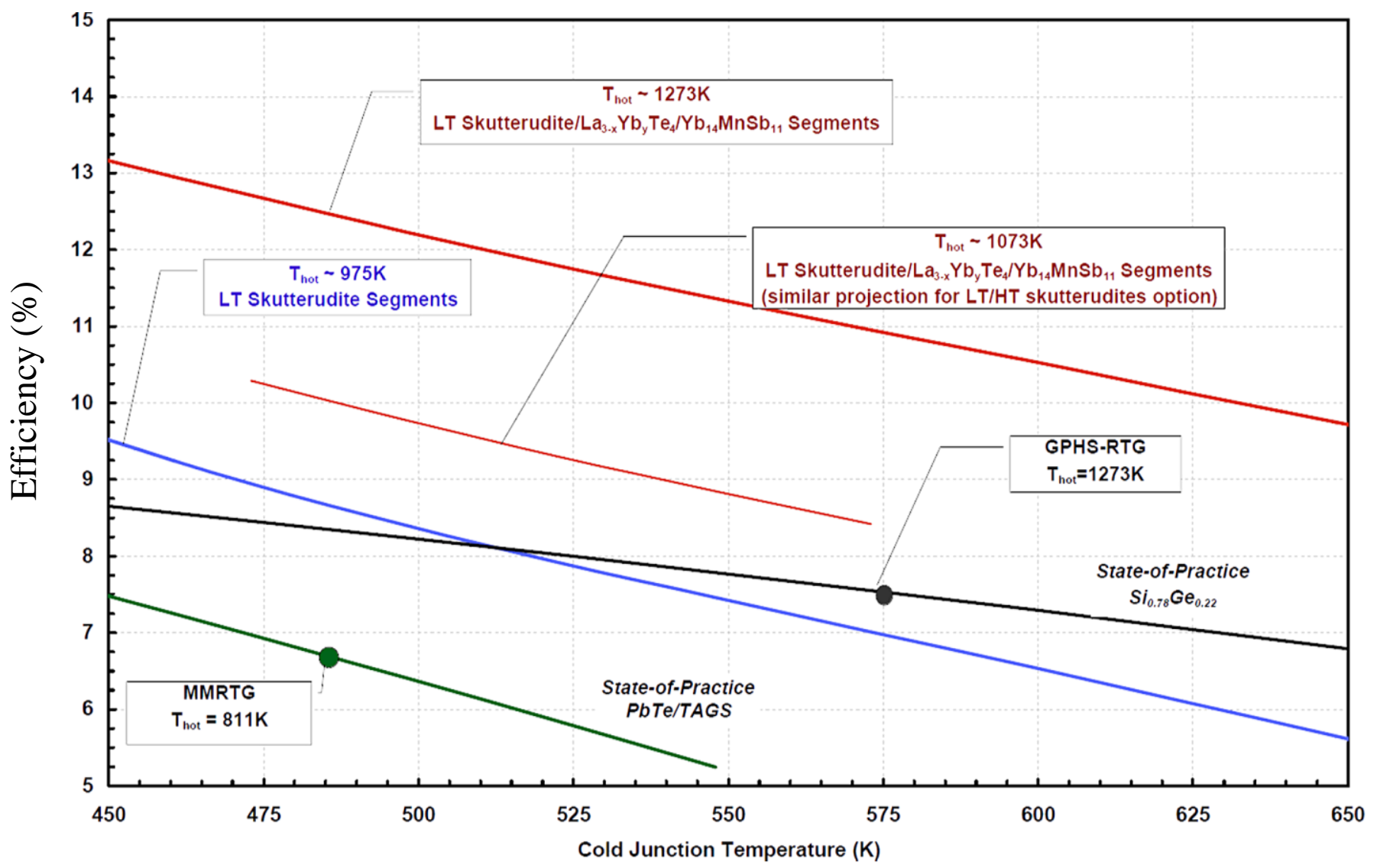

Figure 1.4. Efficiency of various material systems vs $T_{c}[13]$.

\subsection{Material Systems}

Many material systems exhibit thermoelectricity. Reviews by Kanatzidis et al. [18], Snyder et al. [19], and Tritt [20] offer excellent overviews, but the general systems and top performers are outlined here. Some of the material systems include (with examples and short description):

- Chalcogenide $\mathrm{Bi}_{2} \mathrm{Te}_{3}, \mathrm{Sb}_{2} \mathrm{Te}_{3}, \mathrm{PbTe}$

- typically contains Se or Te (chalcogenide) and another heavy slightly more electropositive element, where the layered crystal structure has low thermal conductivity.

- TAGS $(\mathrm{GeTe})_{0.85}\left(\mathrm{AgSbTe}_{2}\right)_{0.15}$

- name from $\mathbf{T e} \mathbf{A g G e S b}$; similar to chalcogenide; limited temperature stability.

- Zintl [valence balanced semiconductor, before doping]

- SiGe cubic structure

- large atomic mass variance decreases thermal conductivity

- $\mathrm{Yb}_{14} \mathrm{MnSb}_{11} \quad \mathrm{~A}_{14} \mathrm{MPn}_{11}$ type; $\mathrm{A}$ and $\mathrm{M}$ are specific metals, $\mathrm{Pn}$ is pnictogen 
- complex structure with tunable electronic properties

- Clathrates $\quad \mathrm{Ba}_{8} \mathrm{Ga}_{16} \mathrm{Ge}_{30}, \mathrm{Sr} 8 \mathrm{Ga} 16 \mathrm{Ge} 30$

- caged structure hosts 'rattler' atoms for scattering thermal conduction.

- Skutterudite $\mathrm{CoSb}_{3}, \mathrm{CeFe}_{4} \mathrm{Sb}_{12}$

- cubic structure of transition metal and pnictogen which also uses rattling filler ions to scatter phonons.

- Half-Heusler $\quad \mathrm{Hf}_{0.6} \mathrm{Zr}_{0.4} \mathrm{NiSn}_{0.99} \mathrm{Sb}_{0.01}, \mathrm{MgAgAs}$-type

- intermetallic compound with interpenetrating FCC sublattices and many vacancies.

- Oxides $\mathrm{NaCO}_{2} \mathrm{O}_{4}, \mathrm{Ca}_{3} \mathrm{Co}_{4} \mathrm{O}_{9}$

- ionic bonding with expected high temperature stability; $\mathrm{CoO}_{2}$ type layer improves electron transport.

- Metal Silicide $\quad \mathrm{FeSi}_{2}, \mathrm{Mg}_{2} \mathrm{Si}$, RareEarth- $\mathrm{B}_{44} \mathrm{Si}_{2}$

- semiconducting compositions using cheaper and less toxic elements.

The common theme among materials is a) balancing electrical conductivity with thermopower, b) lower thermal conduction by complex atomic structures and atomic mass differences, and c) improving mechanical and chemical stability. Performances of some material systems as a function of temperature are given in Figure 1.5. Bismuth telluride is an often used material at room temperature, but at the highest temperatures $\mathrm{Si}-\mathrm{Ge}$ has been the preferred system. The current drive to enhance ZT has compelled researchers to try many methods of material manipulation to increase the thermopower, electrical conductivity, and thermal resistivity; these methods include nanostructuring, Density Of States (DOS) band engineering, modulation doping, and choosing composition from calculations of atomic displacement parameters of rattlers.
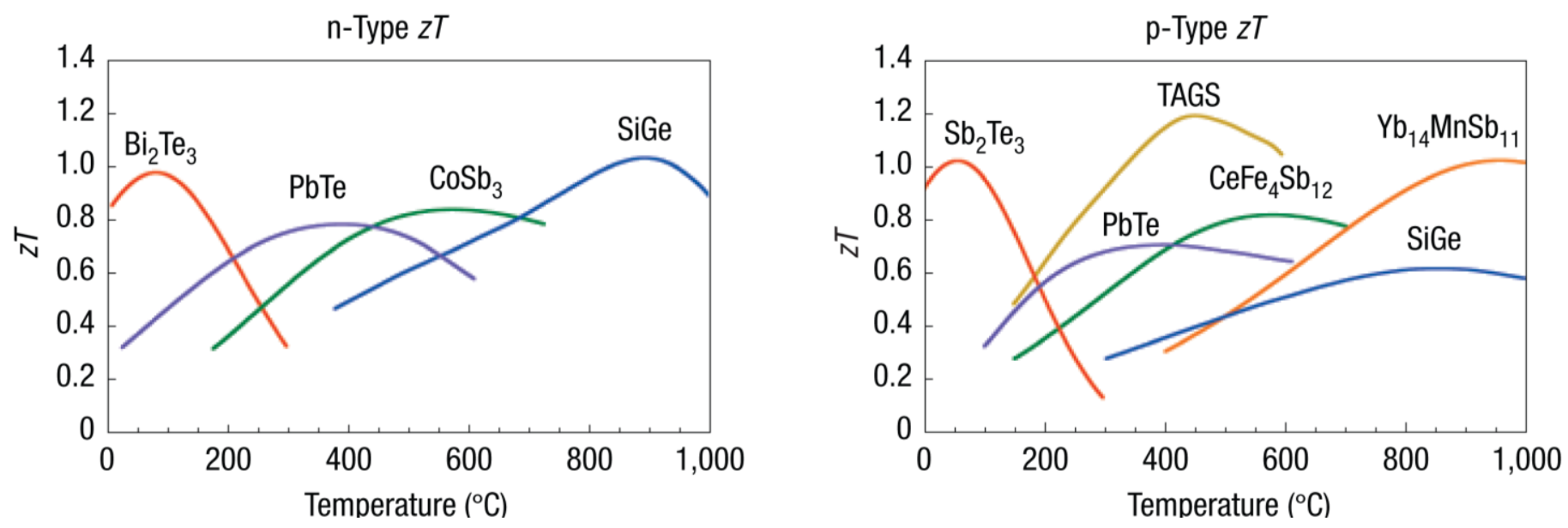

Figure 1.5. ZT of various TE materials, each having an optimum temperature range [19]. 


\subsection{Si-Ge Material System}

The Si-Ge thermoelectric system was chosen because:

$>\mathrm{Si}$, the majority component, is readily available

$>$ Ge concentration can be minimized to reduce raw material costs [21]

$>$ nanostructuring can significantly reduce the high bulk thermal conductivity [22]

$>$ it is non-toxic

$>$ it can be doped with boron or phosphorus to make p- or n-type, respectively

$>$ it has high temperature stability (long-term operation up to $\sim 1000{ }^{\circ} \mathrm{C}$ )

$>$ it has compatibility with waste energy recovery from power plants and engines.

Single-crystal silicon possesses a very high thermal conductivity of $\sim 150 \mathrm{~W} / \mathrm{m} / \mathrm{K}$ at room temperature. Phonon point-defect scattering from alloying with germanium can reduce that value to $\sim 8 \mathrm{~W} / \mathrm{m} / \mathrm{K}$ [23], and with doping impurities the value drops to $\sim 4.5 \mathrm{~W} / \mathrm{m} / \mathrm{K}$ with $\sim 80 \%$ contributed from lattice conduction [17]. Nanostructuring can further reduce the lattice thermal conductivity, possibly down to the $\mathrm{Si}_{0.8} \mathrm{Ge}_{0.2}$ amorphous limit of $\sim 0.9 \mathrm{~W} / \mathrm{m} / \mathrm{K}$ [24]. In fact, lattice thermal conductivity $<2 \mathrm{~W} / \mathrm{m} / \mathrm{K}$ recently was achieved [17]. Reaching the amorphous limit would further improve ZT by $\sim 75 \%$.

Although Si-Ge systems do not currently possess the highest ZT of all TE systems, in inert atmospheres they have long-term stability at high temperatures [25] and are able to operate with a high $\Delta \mathrm{T}$ between hot and cold sides, thus permitting $\eta>10 \%$ [23], with a bulk ideal maximum of $23.3 \%$ [24] at $\Delta \mathrm{T}=1000 \mathrm{~K}$.

Ultimately, the optimal parameters for producing practical thermoelectrics using the silicon-germanium alloy can be taken (to first order at room temperature) as:

- doping level: $\quad>2 * 10^{20} / \mathrm{cm}^{3}$

- composition: $\quad \mathrm{Si}_{0.9} \mathrm{Ge}_{0.1}$ to $\mathrm{Si}_{0.5} \mathrm{Ge}_{0.5}$

- grain size: $\quad \sim 2$ to $30 \mathrm{~nm}$

- mobility: $\quad \mu_{\mathrm{n}}>0.3 \mathrm{~cm}^{2} / \mathrm{V} / \mathrm{s} ; \mu_{\mathrm{p}}>0.2 \mathrm{~cm}^{2} / \mathrm{V} / \mathrm{s}$;

- lattice thermal conductivity: $\kappa_{\mathrm{ph}}<3 \mathrm{~W} / \mathrm{m} / \mathrm{K}$

- efficiency: $\quad \eta>7.5 \%$ to best NASA 


\subsection{Nanoparticles of TE materials and Laser Sintering}

Building thermoelectric materials with nanoparticles takes a bottom-up approach for achieving improved performance of films. By using nanoclusters with a starting grain size of 5-9 $\mathrm{nm}$ prior to laser sintering, it is possible to maximize the density of interfaces after sintering. Nanoparticles absorb light more efficiently than bulk materials and for small enough particle size also exhibit a reduced melting point before the sintering mechanism begins densification.

Laser based manufacturing is continuously growing and is used in automotive, aerospace, photovoltaic and electronic industries. This expansion has been made possible by the availability of high power lasers at decreasing cost and hands-free operation. Laser processing has distinct advantages for high-temperature materials. Lasers can directly heat the sample material to extremely high temperatures, without requiring the sample container to endure those same temperatures. The short duration of laser heating minimizes grain growth during processing [26], thus allowing reduced thermal conductivity in comparison with previous methods such as hot pressing. Additional benefits of employing laser sintering are that the sample can be directly fabricated to near net shape on the desired substrate of choice and that lasers are not confined to working with flat surfaces. Both of these factors may enhance technology transfer to commercial applications. Even the substrate could be sintered before building up the desired coating.

Laser cladding and sintering have been demonstrated for metals, but have not been wellproven for sintering of semiconductors such as $\mathrm{Si}$. Laser processing may reduce cost for fabricating thin films of Si-Ge thermoelectric modules. Likewise, since TE properties vary with temperature and the local temperature varies with position within a TE element, laser sintering layer by layer could allow improved ZT by using functionally graded composition with respect to both germanium and dopant concentration [Figure 2.2, in section 2.2.2]. Furthermore, laser sintering can be applied to nearly any thermoelectric system as most materials absorb energy at typical laser wavelengths. Thus, if an application calls for an average ZT of $>1$ across a large temperature gradient, multiple thermoelectric materials can be segmented in series at a single process stage. Similarly, Si-Ge can be cascaded with $\mathrm{CoSb}_{3}, \mathrm{PbTe}$, and $\mathrm{Bi}_{2} \mathrm{Te}_{3}$ such that each material operates in its optimal temperature range [11]. With the relatively low cost of using a diode laser for sintering, thermoelectric production costs can be minimized to facilitate quicker market penetration. 


\subsection{Problem Statement}

The value of thermoelectric power generation remains too low for large-scale commercialization. Both the cost and efficiency need to be improved to increase their value. Improving efficiency involves optimizing the individual metrics of thermopower, electrical conductivity, and thermal conductivity without impairing another. Nanostructuring offers the best potential for enhancing performance by increasing thermopower and thermal resistivity without considerably reducing electrical conductivity. In this work, a bottom-up approach is taken for achieving nanostructured thin films by using nanoclusters with a starting grain size of $5-9 \mathrm{~nm}$. Laser processing allows extremely fast heating and cooling rates, which permits achieving minimal grain growth of the nanoclusters, as well as the potential for simpler fabrication and lower processing costs. The aim is for an extremely low thermal conductivity, near the amorphous limit of $\sim 1 \mathrm{~W} / \mathrm{m} / \mathrm{K}$ for $\mathrm{Si}-\mathrm{Ge}$, while still maintaining high thermopower and electrical conductivity. Reducing thermal conductivity is the key method for improving the cost performance $(\$ / W)$ of TE power generation [27]. Furthermore, even NASA has settled for second-best TEG materials because the time and cost to re-establish a Si-Ge module production line were too high [28]. Continuous-wave laser sintering does not require substantial investment in terms of time or cost and can be applied to many material systems, thus providing a good solution to the processing of TE materials.

\subsection{Research Objectives}

The objective of this research is to investigate laser sintering of silicon-germanium nanoparticles to create a nanostructured thermoelectric layer. Elevated TE performance can be attained by reducing thermal conductivity via nanostructuring. The hypothesis is that the thermal conductivity can be controlled by laser sintering the nanocrystals, minimizing grain growth during processing due to short heating durations. Furthermore, the enormous interfacial area of nanograins causes enhanced phonon scattering and may simultaneously increase the thermopower due to filtering of low energy electrons, producing a higher overall voltage. The goal is to use laser processing to approach the amorphous limit of lattice thermal conductivity without a corresponding loss in electrical conductivity, in other words to create a PGEC. Realization of the objective has been explored through the following steps: 
1) plasma synthesis of Si-Ge nanoparticles of $<20 \mathrm{~nm}$ in size in collaboration with Prof. Kortshagen at the University of Minnesota,

2) p- and n- type doping of Si-Ge nanoparticles during a) plasma synthesis or b) powder mixing,

3) fabrication of thermoelectric devices by laser sintering of $\mathrm{Si}-\mathrm{Ge}$ nanoparticles,

4) fundamental understanding of material and device properties to high temperatures using state of the art characterization facilities for crystal structures and electrical and thermal properties in collaboration with Oak Ridge National Laboratory through a DOE-funded proposal for thermoelectric research,

5) enhanced research due to collaboration with the plasma synthesis group at UMN and Prof. Hopkins' thermal characterization group at UVA.

Continuous characterization of sintering results is necessary to determine the process window. Optimization of processing conditions is iterative to find the most suitable parameters to successfully achieve grain growth reduction and Seebeck coefficient enhancement. Preliminary results were collected at the University of Virginia using both the Microfabrication Laboratories and the Nanoscale Materials Characterization Facility, as well as at ORNL's High Temperature Materials Laboratory (HTML) using the following characterization methods:

\begin{tabular}{|c|c|c|}
\hline SEM: & Morphology & $\rightarrow$ densification; preliminary grain growth \\
\hline EDS: & Composition & $\rightarrow$ oxidation control; alloy composition \\
\hline 4-point probe: & Electrical Conductivity & $\rightarrow$ dopant activation \\
\hline XRD: & Structure & $\rightarrow$ crystallite size and phases; alloying; impurity phases \\
\hline TEM & Morphology & $\rightarrow$ grain features \\
\hline TEM/EDS & Composition & $\rightarrow$ nanopowder spatial distribution \\
\hline
\end{tabular}

In addition, thermal conductivity measurements have been performed in collaboration with Prof. Patrick Hopkins using the Time-Domain ThermoReflectance (TDTR) method recently installed at the University of Virginia. High temperature thermoelectric properties were measured at ORNL's HTML using:

- Ulvac-ZEM3-M8: Temperature dependence of thermopower and electrical conductivity ○ HT-XRD: Temperature dependence of structure; thermal stability 
Using the Ulvac and TDTR measurements, the laser sintered Si-Ge thermoelectric material figure of merit can be evaluated. Finally, this research investigated the effect of sintering conditions on the individual metrics $(\sigma, \mathrm{S}$, and $\kappa)$ and estimates overall performance (ZT) of the device in order to understand how to achieve the best tradeoff among the thermoelectric parameters. The data allows improving the processing conditions and provides scientific understanding. Ultimately, the achieved ZT and efficiency are then compared to state-of-the-art materials to determine market suitability and applicability to other materials.

This research aims to reduce the barrier to wide-scale implementation by simplifying the production process and therefore lowering the cost. This work also targets to better understand the laser sintering process at the nano-scale, including the distribution of constituents and evolution of wetting for the Si-Ge system.

\subsection{Tasks Accomplished}

1) Establish experimental setup for laser sintering with:
a. induction preheating
b. 2 dimensional computerized scanning stage
c. a controlled atmosphere (argon or low vacuum)

2) Determination of critical parameters of nanoclusters films:
a. Si-Ge composition and dopant concentration
b. substrate material and geometry
c. nanocluster size and film thickness

3) Mitigate film quality issues due to substrate:
a. film cracking due to thermal expansion mismatch
b. film discontinuity due to lack of substrate wetting
c. substrate cracking due to thermal strain

4) Achieve low electrical resistivity through:

a. heavy doping

b. improved percolation of sintered layer

5) Optimize laser sintering parameters

6) Fabricate both n- and p- type thermoelectric layers

7) Deposit non-reactive Ag electrical contacts for $\mathrm{S}, \sigma$ measurements to $1000 \mathrm{~K}$.

8) Enable thermal conductivity measurement to $1000 \mathrm{~K}$ by:

a. reducing surface roughness during sintering 
b. lapping surface to obtain specular reflection

c. depositing high-temperature $\mathrm{Pt}$ transducer with $\mathrm{Si}_{3} \mathrm{~N}_{4}$ barrier layer

9) Investigate properties of laser sintered doped nanopowder Si-Ge thin films:
a. morphology
b. composition
c. structure
d. hall mobility
e. electrical conductivity
f. Seebeck coefficient
g. thermal conductivity

10) Estimate material ZT and efficiency from 300 to $1000 \mathrm{~K}$.

11) Provide scientific understanding of measured $\sigma, S$, and $\kappa$ and impact of oxidation

12) Discuss results, potential improvements, and suitability for energy recovery 


\section{Chapter 2 : Thermoelectric Theory}

\subsection{Concept of Operation}

Thermoelectric materials respond to a heat gradient by equalizing entropy through the creation of an electric field gradient. Physically, the charge carriers in the region at higher temperature have a longer mean free path, and thus preferentially drift towards the colder region [20]. This build-up of charge carriers at the cold side results in a charge build-up, which establishes an electric field. If the majority carriers are electrons then the voltage potential is higher at the hot side, whereas for holes the voltage is higher at the cold side. The drifting of charge carriers continues until one of 1) entropy is equalized (thermal plus electronic), 2) the thermal gradient is removed, or 3) indefinitely if an electrical load is applied to allow charge recirculation.

In semiconductors, both electrons and holes contribute with the minority carrier reducing the usable thermopower; for degenerate doping the minority carrier contribution remains insignificant until very high temperatures [20]. The Seebeck coefficient $S(\mu V / K)$ denotes the material performance for this metric [19], which is negative for n-type materials and positive for p-type materials. The type of material is defined by which type of charge carrier is dominate, electrons for n- or holes for p-. Electrons and holes always have a net drift towards the cold side, but the direction of net charge flow (i.e., current) is opposite for $\mathrm{n}$ - and p-. For this reason, to obtain energy from the system each unicouple of n-element and p-element must be connected thermally in parallel but electrically in series. The generated output current is limited by the lower of the 2 elements, whereas the output voltage is the sum of each element's voltage (thermopower times temperature differential). The voltage is higher than the individual element voltages because although the thermopowers are opposite in sign, so is the relative direction of the thermal gradient, thus effectively the double negatives cancel to produce a sum of the electric potentials. 


\subsection{Physics}

\subsubsection{Electrical}

Thermoelectric properties are determined by a few fundamental parameters, these include the effective mass of both holes and electrons, the composition and dopant concentration, the Mean Free Path (MFP) of both carriers and phonons, and the temperature. Using these parameters, the behavior of the other significant thermoelectric properties can be predicted using the definitions and equations which follow. If a variable is defined using subscripts separated by a comma, then the equation applies for both electrons and holes and the appropriate symbol should be chosen for that the type of material (e.g., $N_{c, v}$ is the carrier effective density of states function for either the conduction band for electrons or valence band for holes).

The effective mass of carriers is defined in Eq. 2-A, and the average scattering time is provided in Eq. 2-B. Note that as both $m^{*}$ and $\bar{\tau}$ are difficult to measure, for this work the density of states carrier effective masses are assumed as 0.42 for electrons and 1.5 for holes [29], unless otherwise stated.

$$
\begin{gathered}
m_{e, h}^{*}=\frac{1}{m_{e}} * \frac{e * \bar{\tau}}{\mu} \\
m_{e}=9.11 \mathrm{E}-31 \mathrm{~kg}, e=1.6 \mathrm{E}-19 \mathrm{~A}^{*} \mathrm{~s}, \bar{\tau} \text { : average carrier scattering time }(\mathrm{s}), \\
\mu: \text { carrier mobility }\left(\mathrm{m}^{2} / \mathrm{V} / \mathrm{s}\right) .
\end{gathered}
$$

Eq. 2-A. Effective mass of charge carriers [30].

$$
\begin{gathered}
\bar{\tau}=l_{e} * \frac{1}{2 * \sqrt{3 * k_{B} * T /\left(m^{*} * m_{e}\right)}} \\
l_{e}=\operatorname{carrier} \operatorname{MFP}(\mathrm{m}), e=1.6 \mathrm{E}-19 \mathrm{~A}^{*} \mathrm{~s}, m_{e, h}^{*}: \text { density of states carrier effective mass }(\mathrm{kg}), \\
k_{B}=1.38 \mathrm{E}-23 \mathrm{~J} / \mathrm{K}, T: \text { temperature }(\mathrm{K}) .
\end{gathered}
$$

Eq. 2-B. Carrier average scattering time. 


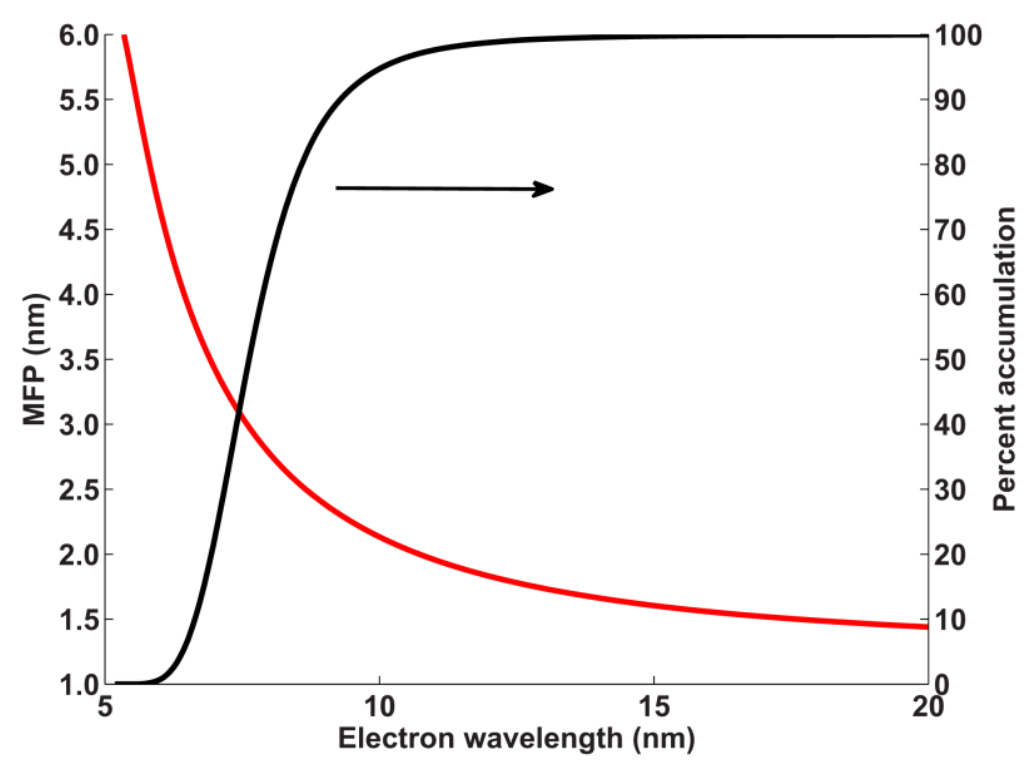

Figure 2.1. $\mathrm{n}-\mathrm{Si}_{0.8} \mathrm{Ge}_{0.2}$ electron MFP vs. wavelength at $300 \mathrm{~K}$, doping of $2 * 10^{20} / \mathrm{cm}^{3}$ [17].

Figure 2.1 graphs Minnich et al.'s modeling of Si-Ge electron MFP as a function of electron wavelength, where the average MFP is $\sim 2.5 \mathrm{~nm}$ at room temperature [17].

Electron mobility is given by the classical definition in Eq. 2-C, however that definition only accounts for lattice vibrations. Thus, an improved model incorporates the carrier effective mass, as in Eq. 2-D.

$$
\begin{gathered}
\mu \approx \frac{4}{3} * e * l_{e} *\left(2 * \pi * m_{e} * k_{B} * T\right)^{-1 / 2} \\
e=1.6 \mathrm{E}-19 \mathrm{~A}^{*} \mathrm{~s}, l_{e}: \text { electron MFP }(\mathrm{m}), m_{e}=9.11 \mathrm{E}-31 \mathrm{~kg}, k_{B}=1.38 \mathrm{E}-23 \mathrm{~J} / \mathrm{K}, T: \text { temperature }(\mathrm{K}),
\end{gathered}
$$

Eq. 2-C. Electrical mobility dependencies, classical definition [31].

$$
\mu^{*} \approx \mu *\left(m_{e, h}^{*}\right)^{3 / 2}
$$

$\mu$ : carrier mobility (m²/V/s), $m_{e, h}^{*}$ : density of states carrier effective mass (unitless)

Eq. 2-D. Weighted electrical mobility [32].

Using the carrier effective mass and the temperature, the carrier effective density of states function can be calculated by Eq. 2-E, assuming a parabolic band [33]. Then, the active carrier 
concentration can be calculated using the Fermi-Dirac (FD) integral given the scattering parameter (s) and reduced Fermi energy (defined in Eq. 2-N in section 2.2.2).

$$
N_{c, v} \approx \frac{2 *\left(2 * \pi * m_{e, h}^{*} * m_{e} * k_{B} * T\right)^{3 / 2}}{h^{3}}
$$

$m_{e, h}^{*}:$ density of states carrier effective mass (unitless), $m_{e}=9.11 \mathrm{E}-31 \mathrm{~kg}, k_{B}=1.38 \mathrm{E}-23 \mathrm{~J} / \mathrm{K}$,

$$
T \text { : temperature }(\mathrm{K}), h=6.63 \mathrm{E}-34 \mathrm{~J}^{*} \mathrm{~s} \text {. }
$$

Eq. 2-E. Calculation of the effective carrier density of states function $\left(N_{c}\right.$ : electron density, $N_{v}$ : hole density).

$$
n \approx N_{c, v} * \mathcal{F}_{S+1 / 2}\left(\eta_{F}\right)
$$

$N_{c, v}$ : carrier effective DOS function $\left(\mathrm{m}^{-3}\right), \mathcal{F}_{\mathrm{n}}(\eta)$ : Fermi-Dirac integral of order $\mathrm{n}$ (here $\mathrm{n}=\mathrm{s}+1 / 2$ ),

$\eta_{F}$ : reduced Fermi energy $\left(\eta_{F}=\left(E_{F}-E_{c}\right) / k_{B} T\right.$ or $\left.\left(E_{v}-E_{F}\right) / k_{B} T\right)$, s: scattering parameter, $E_{c, v}$ : energy of band edge

Eq. 2-F. Calculation of carrier concentration from the reduced Fermi energy [34].

$$
\begin{array}{ccc}
\text { The gamma function for } \eta>0 \text { is: } & \Gamma(\eta) & =\int_{0}^{\infty} \varepsilon^{\eta-1} e^{-\varepsilon} d \varepsilon \\
\text { The Fermi-Dirac (FD) integral for } \eta>0 \text { is: } & \mathcal{F}_{n}(\eta)=\frac{1}{\Gamma(n+1)} * \int_{0}^{\infty} \frac{t^{n}}{e^{\varepsilon-\eta}} d \varepsilon \\
\varepsilon \text { : energy of charge carrier. }
\end{array}
$$

Eq. 2-G. Definition of the gamma function and Fermi-Dirac integral.

Care must be taken when using the FD integral, as literature sometimes removes the $1 /(\Gamma(n+$ 1)) and instead adds a static value of this gamma function (e.g., $2 / \sqrt{\pi}$ for $n=0.5$ ) to the equation which uses the integral. The reduced Fermi energy is the difference between the Fermi energy and carrier band edge $\left(E_{c, v}\right)$, normalized by the thermal energy $\mathrm{k}_{\mathrm{B}} \mathrm{T}$ (e.g., $\eta_{F}=\left(E_{F}-E_{c}\right) / k_{B} T$ or $\left.\eta_{F}=\left(E_{v}-E_{F}\right) / k_{B} T\right)$. The order of the FD integral (n) is determined by the scattering parameter (s) for thermoelectric evaluation. The scattering parameter value depends upon the dominant type of carrier scattering and ranges from $-1 / 2$ to $+3 / 2$, and equals $-1 / 2,1 / 2$, or $3 / 2$ for pure scattering by acoustic phonons, optical phonons, or ionized impurities, respectively [35]. 'Acoustic' scattering is also called 'lattice' or 'phonon'; 'optical' is also called 'ionic'; 'lattice' and 'ionic' definitions of scattering offer an alternative understanding of the physical reasons the carriers experience scattering events. For degenerate $\mathrm{Si}-\mathrm{Ge}$ at room temperature $\mathrm{s} \approx 1 / 2$ for both electrons and holes; $(\mathrm{s}+1 / 2)$, defined as $R$, gives the temperature-dependent exponent of the 
carrier mean free path (MFP) [24], which also indicates that the carrier MFP is approximately proportional to the carrier energy to the power of $R$ [36].

$$
R=s+\frac{1}{2}
$$

s: carrier scattering value (-0.5: Lattice, 0.5: Optical, 1.5: lonized Impurity)

Eq. 2-H. Scattering parameter R gives the exponent of carrier scattering temperature dependence.

Finally, the electrical conductivity can then be determined using Eq. 2-I.

$$
\begin{gathered}
\sigma=n * e * \mu^{*} \\
\mathrm{n} \text { : carrier density }\left(\mathrm{m}^{-3}\right), e=1.6 \mathrm{E}-19 \mathrm{~A}^{*} \mathrm{~s}, \mu^{*} \text { : weighted carrier mobility }\left(\mathrm{m}^{2} / \mathrm{V} / \mathrm{s}\right)
\end{gathered}
$$

Eq. 2-I. Electrical conductivity dependencies.

Another electrical property which can vary significantly for thermoelectrics is the Lorenz number. The Lorentz number $(\mathscr{L})$ depends upon the charge carrier scattering mechanisms [37], level of doping and curvature of the density of states [38]. $\mathscr{L}$ for low temperatures or metallic doping should equal the Sommerfeld value [39]:

$$
L_{0}=\frac{\pi^{2} * k_{B}{ }^{2}}{3 * e^{2}}=2.45 * 10^{-8} V^{2} K^{-2}
$$

Eq. 2-J. Sommerfeld value of Lorentz number.

$\mathscr{L}$ can be modeled using Fermi-Dirac statistics as shown in Eq. 2-K, dependent on the reduced Fermi energy and the scattering parameter (s). For bulk Si-Ge alloy, Zhu et al. modeled $\mathscr{L}$ to be from $2.2 \rightarrow 1.3$ from $25^{\circ} \mathrm{C} \rightarrow 1000{ }^{\circ} \mathrm{C}$, with 0.2 variation at a given temperature when degenerately doped [21]. Note that experimental values provided for $\mathscr{L}$ typically don’t include the ambipolar contribution. 


$$
\begin{gathered}
L=\frac{k_{B}{ }^{2}}{e^{2}} *\left[\frac{\Gamma\left(s+\frac{9}{2}\right) * \mathcal{F}_{s+5 / 2}\left(\eta_{F}\right)}{\Gamma\left(s+\frac{5}{2}\right) * \mathcal{F}_{s+1 / 2}\left(\eta_{F}\right)}-\left(\frac{\Gamma\left(s+\frac{7}{2}\right) * \mathcal{F}_{s+3 / 2}\left(\eta_{F}\right)}{\Gamma\left(s+\frac{5}{2}\right) * \mathcal{F}_{s+1 / 2}\left(\eta_{F}\right)}\right)^{2}\right] \\
k_{B}=1.38 \mathrm{E}-23 \mathrm{~J} / \mathrm{K}, e=1.6 \mathrm{E}-19 \mathrm{~A}^{*} \mathrm{~s}, \Gamma(\mathrm{x}): \text { gamma function; s: scattering parameter; } \\
\mathcal{F}_{\mathrm{n}}(\eta): \text { Fermi-Dirac integral of order } \mathrm{n}, \eta_{F} \text { : reduced Fermi energy. }
\end{gathered}
$$

Eq. 2-K. Lorentz number dependence on scattering parameter and reduced Fermi energy [40].

\subsubsection{Doping}

The carrier concentration $n$ is determined by the doping level and degree of activation (i.e., ionization). The thermopower dependents heavily upon the Fermi energy and thus can be controlled by the material composition and doping level [41]. For Si-Ge thermoelectric systems, extensive literature has shown the best performance occurs at degenerate doping levels with a carrier concentration near $10^{20} / \mathrm{cm}^{3}$. Dopant atoms may be added at much higher concentrations ( $>1$ at \%), however not all of the dopants are activated, especially for material with nano-sized grains. Experience with the $\mathrm{Si}-\mathrm{Ge}$ system shows that the active doping concentration must be within $\sim 40 \%$ of ideal or else ZT will fall by more than 3\% [24]. Figure 2.2 shows that the optimum carrier density is a function of temperature, thus to achieve the best average ZT the material should be functionally graded to have the dopant concentration lower at the cold side and higher at the hot side. 


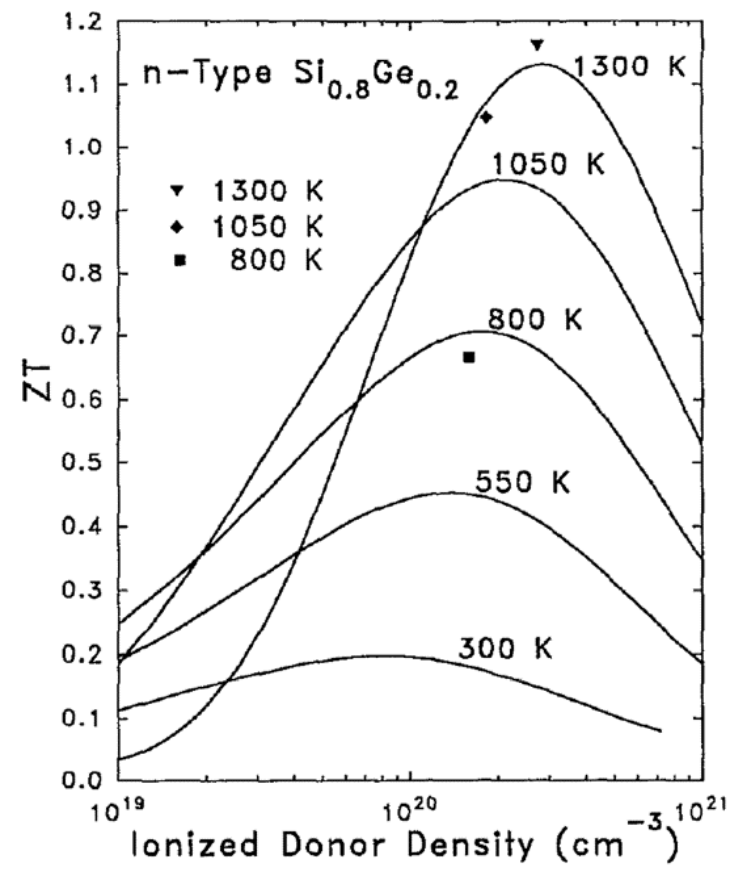

Figure 2.2. ZT dependence of Si-Ge on ionized carrier density at various temperatures [42].

Degenerate doping occurs when the Fermi energy $\left(\mathrm{E}_{\mathrm{F}}\right)$ is within $3 \mathrm{k}_{\mathrm{B}} \mathrm{T}$ of either band edge [43].

$$
E_{F} \leq E_{v}+3 k_{B} T \quad \text { or } \quad E_{F} \geq E_{c}-3 k_{B} T
$$

$E_{F}$ can be calculated by combining Eq. 2-L, Eq. 2-M, and Eq. 2-N, given $E_{\mathrm{G}}($ section 5.2.5) and the carrier effective masses (section 2.2.1). In the degenerate case, general semiconductor approximations are not accurate [35]. Instead, improved approximations (e.g., E F $_{\text {using }}$ Nilsson's approximation) or Fermi-Dirac integrals must be used to find numerical solutions. The intrinsic energy level is defined in Eq. 2-M. Using the bandgap, carrier effective mass, and the carrier concentration, the reduced Fermi energy can be calculated using Nilsson approximation as shown in Eq. 2-N, which keeps error to < 1\% [44].

$$
E_{c}=\frac{E_{G}}{2}-E_{i}, \quad \eta_{F}=\left(E_{F}-E_{c}\right) * \frac{e^{*}}{k_{B} * T}
$$

$E_{G}$ : bandgap (eV), $E_{i}$ : intrinsic energy $(\mathrm{eV}), k_{B}=1.38 \mathrm{E}-23 \mathrm{~J} / \mathrm{K}, T$ : temperature $(\mathrm{K}), e^{*}=1.6 \mathrm{E}-19 \mathrm{~J} / \mathrm{eV}$. Eq. 2-L. Definition of the Fermi energy $\left(E_{F}\right)$ and the reduced Fermi energy $\left(\eta_{F}\right)$. 


$$
E_{i}=\frac{3}{4} * \frac{k_{B} * T}{e^{*}} * \ln \left(\frac{m_{h}^{*}}{m_{e}^{*}}\right)
$$

$m_{h}^{*}$ : density of states hole effective mass, $m_{e}^{*}$ : density of states electron effective mass,

$$
k_{B}=1.38 \mathrm{E}-23 \mathrm{~J} / \mathrm{K}, \quad T \text { : temperature }(\mathrm{K}), e^{*}=1.6 \mathrm{E}-19 \mathrm{~J} / \mathrm{eV} .
$$

Eq. 2-M. Definition of the intrinsic energy level $E_{i \text {. }}$

$$
\eta_{F} \approx \frac{\ln \left(n / N_{c, v}\right)}{\left(1-n / N_{c, v}\right)}+\left(\frac{3 * \sqrt{\pi}}{4} * \frac{n}{N_{c, v}}\right)^{2 / 3}+\frac{3 / 2 * \sqrt{\pi} * n / N_{c, v}}{\left(3+3 / 4 * \sqrt{\pi} *\left(n / N_{c, v}\right)\right)^{2}}
$$

$n$ : carrier density $\left(\mathrm{m}^{-3}\right), N_{c, v}:$ carrier effective DOS function $\left(\mathrm{m}^{-3}\right), k_{B}=1.38 \mathrm{E}-23 \mathrm{~J} / \mathrm{K}$.

Eq. 2-N. Calculation of the reduced Fermi energy $\left(\eta_{F}\right)$ using Nilsson's approximation [44].

\subsubsection{Thermopower (S)}

Once the electronic properties of the material are defined, Eq. 2-O can be used to estimate $\mathrm{S}$ for nearly free electrons [45]. This energy-independent scattering approximation assumes a single parabolic carrier band at high $n$ and/or low $\mathrm{T}$ [32]. The parameter $\mathrm{R}$ is the scattering factor defined in section 2.2.1 as the temperature dependence of carrier scattering. An alternative definition is provided in Eq. 2-P to show the relation to the band edge energy. The optimal doping concentration $n$ can be estimated (to a first-order approximation) by locating the peak power factor in the temperature range of interest [46]. 


$$
\begin{gathered}
S_{e, h} \approx \frac{\pi^{2} * k_{B}^{2} * T}{3 * e} * \frac{8 * m_{e, h}^{*} * m_{e}}{h^{2}} *\left(\frac{\pi}{3 * n}\right)^{2 / 3} *(1+R) \\
\text { where } \mathrm{S} \text { is negative for n-type and } R=\left(\frac{d \ln l_{e}}{d \ln E}\right)_{E_{F}} \\
k_{B}=1.38 \mathrm{E}-23 \mathrm{~J} / \mathrm{K}, T: \text { temperature }(\mathrm{K}), m_{e, h}^{*}: \text { density of states carrier effective mass }(\mathrm{kg}), \\
m_{e}=9.11 \mathrm{E}-31 \mathrm{~kg}, h=6.63 \mathrm{E}-34 \mathrm{~J}^{*} \mathrm{~s}, n: \text { carrier density }\left(\mathrm{m}^{-3}\right), e=1.6 \mathrm{E}-19 \mathrm{~A}^{*} \mathrm{~s}, \\
l_{e}=\text { carrier MFP }(\mathrm{m}), E: \text { carrier energy }(\mathrm{J}), E_{F}: \text { Fermi energy }(\mathrm{J}), R: \text { scattering parameter. }
\end{gathered}
$$

Eq. 2-0. Seebeck coefficient dependence on Fermi energy for nearly free electrons [45].

$$
\begin{gathered}
S_{e, h} \approx \frac{k_{B}}{e} *\left(\frac{E_{c, v}}{k_{B} * T}-A+\ln \left(\frac{2 *\left(2 * \pi * m_{e, h}^{*} * m_{e} * k_{B} * T\right)^{3 / 2}}{h^{3} * n}\right)\right) \\
k_{B}=1.38 \mathrm{E}-23 \mathrm{~J} / \mathrm{K}, e=1.6 \mathrm{E}-19 \mathrm{~A}^{*} \mathrm{~s}, T: \text { temperature }(\mathrm{K}), n: \text { carrier density }\left(\mathrm{m}^{-3}\right), \\
m_{e, h}^{*}: \text { density of states carrier effective mass }(\mathrm{kg}), m_{e}=9.11 \mathrm{E}-31 \mathrm{~kg}, h=6.63 \mathrm{E}-34 \mathrm{~J}^{*} \mathrm{~s}, \\
E_{c, v}: \text { band edge energy }(\mathrm{J}), A \text { : material-dependent constant for carrier scattering. }
\end{gathered}
$$

Eq. 2-P. Alternative Seebeck coefficient definition [47].

Numerous methods have been proposed to enhance the Seebeck coefficient; the enhancements can be more easily understood by relating the thermopower to the average carrier energy as in Eq. 2-Q.

$$
\begin{gathered}
S \approx \frac{e^{*}}{e} *\left(\frac{\bar{E}-E_{F}}{T}\right) \\
e^{*}=1.6 \mathrm{E}-19 \mathrm{~J} / \mathrm{eV}, e=1.6 \mathrm{E}-19 \mathrm{~A}^{*} \mathrm{~s}, T: \text { temperature }(\mathrm{K}), \\
\bar{E}: \text { average charge carrier energy, } E_{F}: \text { Fermi energy. }
\end{gathered}
$$

Eq. 2-Q. Seebeck coefficient relation to carrier energy and $E_{F}[\mathbf{3 6}]$. 


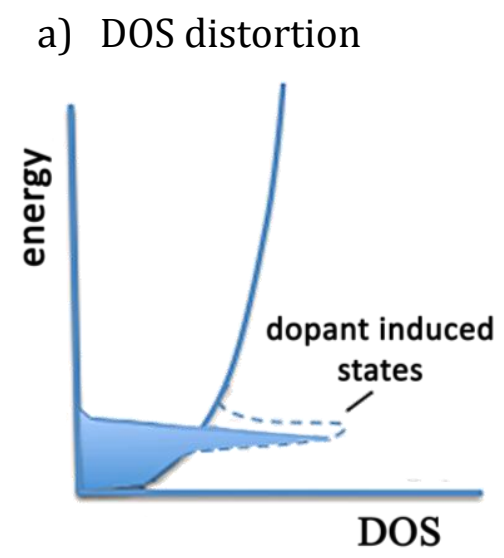

b) quantum-confinement

c) energy filtering
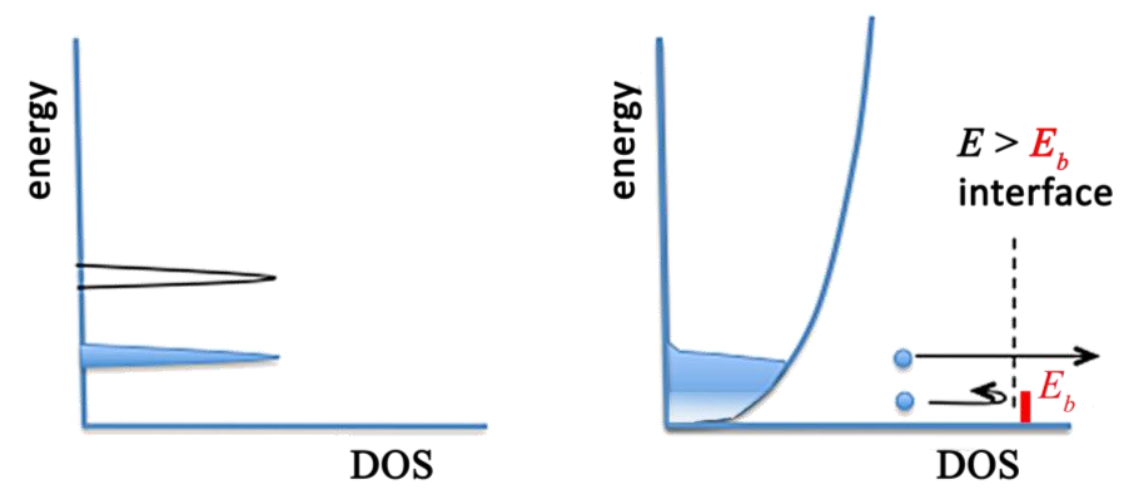

Figure 2.3. Seebeck enhancment methods [48].

Seebeck coefficient improvements can occur from grain boundary electron energy filtering [7,49], quantum size effects [50,51], and increased DOS at the Fermi level [9]. Enhancement of the DOS near the Fermi level can allow more carrier states at higher energy levels, thus increasing the average energy per charge carrier, $\bar{E}$, and improving the Seebeck coefficient [52]. Likewise, the use of quantum confinement to allow conducting states only near $E_{F}$ also increases the average carrier energy and therefore provides improved Seebeck coefficient. Energy filtering occurs when the carrier states of lower energy are inhibited by energy barriers such as grain boundaries, giving them lower mobility and therefore less contribution to the average. Again, having fewer lower-energy carriers increases the value of $S$. Figure 2.3 depicts these enhancements schematically. Furthermore, band engineering to increase the DOS just above $E_{F}$ also improves the value of $S$, as the higher energy electrons have a longer MFP; this condition increases the scattering parameter R, as defined in Eq. 2-O [53].

Phonon drag is another condition for increasing $S$, contributing $\mathrm{S}_{\mathrm{drag}}$. The increase in $S$ occurs because at certain temperatures and carrier concentrations in some materials, the phononelectron interaction tends to push electrons to the cold end of the material [36]. At very low temperatures, too few phonons are available to cause this effect [54], and at higher temperatures or high vacancy concentrations, lattice imperfections permit scattering by Umklapp processes to satisfy Bloch conditions [45]. $S_{\text {drag }}$ reaches a maximum near $\theta_{\mathrm{D}} / 5$, one fifth of the Debye temperature [55]. Above $\theta_{\mathrm{D}} / 5$, however, the phonons lose momentum through phonon-phonon scattering instead of phonon-electron scattering [54]. For pure silicon, phonon-drag is absent 
above $10^{18} / \mathrm{cm}^{3}$ or $>300 \mathrm{~K}$ [56], whereas for pure germanium the temperature is even lower at $>180 \mathrm{~K}$ [57]. For Si-Ge, phonon drag is not observed at or above room temperature due to the relatively short phonon mean free path [24].

\subsubsection{Thermal}

Thermal conductivity is contributed to by both phonons and charge carriers, as defined in Eq. 2-R. The portion from the charger carriers is further split between the majority carrier (electronic) and ambipolar (electron-hole pair) contributions.

$$
\begin{gathered}
\kappa=\kappa_{p h}+\left(\kappa_{e}+\kappa_{b p}\right) \\
\kappa_{p h}: \text { phonon/lattice, } \kappa_{e}: \text { electronic, } \kappa_{b p}: \text { ambipolar }
\end{gathered}
$$

Eq. 2-R. Thermal conductivity components.

The electronic portion is classically described by the Wiedemann-Franz law (which assumes no minority carriers, such that $\kappa_{b p}$ is zero), in Eq. 2-S.

$$
\kappa_{e}=L * T * \sigma
$$

$\mathscr{L}(\mathrm{L})$ : Lorentz number $\left(\mathrm{V}^{2} / \mathrm{K}^{2}\right), \mathrm{T}$ : Temperature $(\mathrm{K}), \sigma$ : electrical conductivity $(1 / \Omega / \mathrm{m})$

Eq. 2-S. Electronic thermal conductivity dependencies.

The Wiedemann-Franz law is the accepted method to calculate the electronic contribution to the thermal conductivity for metals, however for non-metals it should be used only as an approximation if the Lorentz number has not been accurately determined. For example, as shown in Figure 2.4, Vining modeled that at high temperature there is a minimum $\kappa_{\mathrm{e}}$ for doped Si-Ge near $10^{20}$ carriers $/ \mathrm{cm}^{3}$ [42]. $\kappa_{\mathrm{e}}$ usually decreases with lower doping levels, but for Si-Ge there is a significant ambipolar diffusion contribution to the Lorenz number at doping levels $<10^{20}$ [42]. Ambipolar diffusion occurs when the Fermi energy $\mathrm{E}_{\mathrm{F}}$ crosses more than one bandedge [35], which also occurs for small bandgap materials at high temperatures [36]. Heat is transferred but since the electrons and holes recombine there is no net charge transfer, thus from the classical definition where $\kappa_{e}$ must include any non-zero $\kappa_{b i}$ then $\mathscr{L}>L_{0}$, making it difficult to determine solely the lattice contribution to $\kappa$. On the other hand, at lower temperatures $\mathscr{L}$ can be $<L_{0}$ for non-degenerate doping or even approach zero if the DOS resembled a delta function [38]. Moreover, when electrons are confined to 1D systems the Wiedemann-Franz law can 
become invalid [58]. Thus, using Eq. 2-K with the model of scattering parameter vs. temperature (section 5.2.3) provides better accuracy.

The ambipolar contribution to thermal conduction can be modeled using the individual contributions of the majority and minority carriers, using Eq. 2-T.

$$
\begin{gathered}
\kappa_{b p} \approx \frac{\left(\sigma_{e} * \sigma_{h}\right)}{\left(\sigma_{e}+\sigma_{h}\right)} *\left(S_{e}-S_{h}\right)^{2} * T \\
e: \text { electron, } h: \text { hole }
\end{gathered}
$$

Eq. 2-T. Ambipolar thermal conduction using $S_{e}$ and $S_{h}$ [59].

Phonon (or lattice, $\kappa_{1}$ ) thermal contribution is given by the classical equation in Eq. 2-U.

$$
\kappa_{p h}=\frac{1}{3} * C_{v} * v_{D} * \ell_{p h}
$$

$C_{v}$ : heat capacity $\left(\mathrm{J} / \mathrm{m}^{3} / \mathrm{K}\right), \nu_{D}$ : Debye speed of sound $(\mathrm{m} / \mathrm{s}), \ell_{p h}$ : phonon mean free path $(\mathrm{m})$,

Eq. 2-U. Lattice thermal conductivity.

Which for low $\mathrm{T}$ (less than $\sim 10 \mathrm{~K}$ for SiGe) obeys the Debye $\mathrm{T}^{3}$ law, given in Eq. 2-V.

$$
\begin{gathered}
\kappa_{p h}=\frac{2 \pi^{2}}{5} * \frac{k_{B}^{4}}{\hbar^{3} v_{D}{ }^{3}} * v_{D} * T^{3} * \ell_{p h} \\
k_{B}=1.38 \mathrm{E}-23 \mathrm{~J} / \mathrm{K}, \nu_{D} \text { : Debye speed of sound }(\mathrm{m} / \mathrm{s}), \ell_{p h} \text { : phonon mean free path }(\mathrm{m}), \\
\hbar=1.06 \mathrm{E}-34 \mathrm{~J}^{*} \mathrm{~s}, T: \text { temperature }(\mathrm{K})
\end{gathered}
$$

Eq. 2-V. Lattice thermal conductivity at low temperatures.

A model proposed by Lui et al. provides different dependencies, in this case it requires the average mass per atom, average atomic volume, Debye temperature, temperature, and gruneisen parameter, given in Eq. 2-W [59]. The gruneisen parameter for Si-Ge is 1.01 [29]. 


$$
\kappa_{p h}=\frac{3.5 * k_{B}}{h} * \frac{M * V^{1 / 3} * \theta_{D}^{3}}{\gamma^{2} * T}
$$

$k_{B}=1.38 \mathrm{E}-23 \mathrm{~J} / \mathrm{K}, M$ : average atom mass $(\mathrm{kg}), \mathrm{V}$ : average atomic volume $\left(\mathrm{m}^{3}\right)$,

$\theta_{\mathrm{D}}$ : Debye speed of sound $(\mathrm{m} / \mathrm{s}), h=6.63 \mathrm{E}-34 \mathrm{~J}^{*} \mathrm{~s}, \gamma$ : gruneisen parameter, $T$ : temperature $(\mathrm{K})$, Eq. 2-W. Lattice thermal conductivity using atomic dependencies [59].

At temperatures $>\sim 300 \mathrm{~K}$, the speed of sound $\left(\nu_{D}\right)$ essentially does not have a temperature dependence, whereas the phonon mean free path $\left(\ell_{p h}\right)$ continues decreasing as temperature exceeds the Debye temperature $\left(\theta_{\mathrm{D}}\right)[20] . \ell_{p h}$ is given by phonon group velocity multiplied by the phonon relaxation time. These parameters can be modeled using methods such as densityfunctional perturbation theory [60] and the phonon Boltzmann transport equation [17]. Although the Boltzmann equation is not expected to be reliable when the electron MFP is less than the wavelength, the model still provides a good fit to experimental values [17].

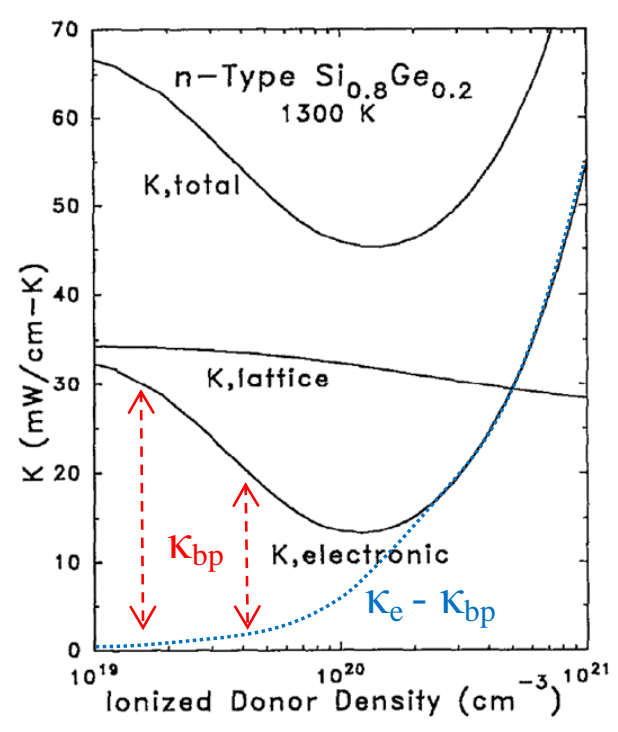

Figure 2.4. Si-Ge thermal conductivity dependence on carrier density at $1300 \mathrm{~K}$ [42].

Vining modeled the thermal conductivity of $\mathrm{n}-\mathrm{Si}_{0.8} \mathrm{Ge}_{0.2}$ as a function of doping level at $1300 \mathrm{~K}$. As shown in Figure 2.4, the graph separates the phonon part from the electronic part, and the ambipolar portion of the electronic part is evident below $2 * 10^{20} / \mathrm{cm}^{3}$ doping density. Zamanipour et al. show the $\kappa_{\mathrm{bp}}$ contribution for $\mathrm{Si}_{0.8} \mathrm{Ge}_{0.2}$ doped with boron to $2 * 10^{20} / \mathrm{cm}^{3}$ is 
nearly zero until $\sim 800{ }^{\circ} \mathrm{C}$, increasing to $\sim 1 \mathrm{~W} / \mathrm{m} / \mathrm{K}$ by $1000{ }^{\circ} \mathrm{C}$ [61], with higher doping levels reducing the $\kappa_{\mathrm{bp}}$ contribution due to lower minority carrier concentrations. Conversely, lower doping levels will shift the temperature lower to $\mathrm{T}^{*} ; \mathrm{T}^{*}$ can be estimated by where the peak thermopower occurs.

By limiting lattice thermal conductivity, enhanced grain boundary scattering via nanostructuring should allow for reaching ZT $>2$ at the phonon diffuse mismatch limit; this theory states that a phonon scatters at every grain boundary, such that the phonon mean free path equals the grain size [29]. At smooth interfaces this scattering model is not applicable, thus higher grain boundary roughness or other scattering centers are needed.

\subsection{Material Selection}

One of the first theories used to evaluate semiconductors for thermoelectric application was by Goldsmid in 1954 [31]. The equation Eq. 2-X emphasizes the importance of having larger average atomic weight to get better thermoelectric performance, as the mobility to thermal conductivity ratio increases very quickly for heavier atoms due to A increasing, $d$ increasing, and $v_{S}$ decreasing simultaneously.

$$
\frac{\mu}{\kappa} \approx \mathrm{K}^{\prime} * \frac{A * d}{v_{s}}
$$

$A$ : mean atomic weight (AMU), $d$ : density $\left(\mathrm{kg} / \mathrm{m}^{3}\right), \nu_{s}$ : sound velocity $(\mathrm{m} / \mathrm{s}), \mathrm{K}^{\prime}$ : constant, Eq. 2-X. Goldsmid's mobility to thermal conductivity material criteria [31].

A measure of the electron-phonon decoupling necessary for further improving ZT is the quality factor $\beta$, as given by Chasmar and Stratton in 1959 in Eq. 2-Y [62]: 


$$
\beta=\frac{\mu * T *\left(\frac{k_{B}}{e}\right)^{2} * \frac{2 * e *\left(2 * \pi * m^{*} * m_{e} * k_{B} * T\right)^{3 / 2}}{h^{3}}}{\kappa_{p h}}
$$

$\mu$ : carrier mobility ( $\left.\mathrm{m}^{2} \mathrm{~N} / \mathrm{s}\right), T$ : temperature (K), $k_{B}=1.38 \mathrm{E}-23 \mathrm{~J} / \mathrm{K}, e=1.6 \mathrm{E}-19 \mathrm{~A}^{*} \mathrm{~s}$, $m^{*}$ : density of states carrier effective mass $(\mathrm{kg}), m_{e}=9.11 \mathrm{E}-31 \mathrm{~kg}, h=6.63 \mathrm{E}-34 \mathrm{~J}^{*} \mathrm{~s}$, $\kappa_{p h}$ : phonon thermal conductivity $(\mathrm{W} / \mathrm{m} / \mathrm{K})$,

Eq. 2-Y. Chasmar and Stratton's quality factor for TE materials [62].

$\beta$ provides a good starting point for optimal material selection; it emphasizes the importance of having high mobility and carrier effective mass while minimizing lattice thermal conductivity. For instance, ionic compounds have poor mobility due to significant polar scattering of electrons and thus are not typically a good material choice [36].

Another material parameter $\gamma$ was proposed by Simon in 1962, defined in Eq. 2-Z [63]:

$$
\gamma=\left(\frac{m_{e}^{*}}{m_{h}^{*}}\right)^{3 / 4} *\left(\frac{\mu_{e}}{\mu_{h}}\right)^{1 / 2}
$$

$m_{h}^{*}$ : density of states hole effective mass, $m_{e}^{*}$ : density of states electron effective mass, $\mu_{h}$ : hole mobility $\left(\mathrm{m}^{2} \mathrm{~N} / \mathrm{s}\right), \mu_{e}$ : electron mobility $\left(\mathrm{m}^{2} / \mathrm{V} / \mathrm{s}\right)$,

Eq. 2-Z. Simon's quality factor $\gamma$ for TE materials [63].

$\gamma$ equals one for optical phonon scattering, but varies from one for acoustical phonon scattering, which indicates improved material performance ( $\gamma>1$ for $\mathrm{n}$-type, $\gamma<1$ for $\mathrm{p}$-type).

One method used to lower thermal conductivity is by alloying atoms of significantly different atomic mass, as the higher atomic mass sites act as scattering centers and have a lower sound velocity [36]. It was proposed that heavier dopants may be able to act as both a dopant and phonon scattering site, performing better than boron or phosphorus. Dismukes et al., however, reported that doping Si-Ge with arsenic increased thermal conductivity $\sim 5 \%$ over phosphorus rather than decreased it, which in combination with the decreased mobility and solubility of arsenic compared with phosphorus [23] negated significant investigation into using higher atomic mass dopants with Si-Ge. Rather, gallium phosphide has been employed as it also 
increases the solubility of phosphorus [21,64,65], while for p-type Si-Ge, erbium has been shown to improve the activation of boron and possibly Seebeck enhancement [66].

For the silicon-germanium thermoelectric system, germanium's primary function is to reduce the thermal conductivity beyond that of silicon alone. Henry and Chen [67] show that in bulk $\mathrm{Si}$, at room temperature $80 \%$ of thermal conductivity comes from phonons with $<10 \mathrm{~nm}$ wavelengths and that $35 \%$ of heat is transferred by phonons with mean free paths (MFP) greater than $1 \mu \mathrm{m}$. Creating point defects by alloying the Si with Ge preferentially scatters high frequency phonons, reducing heat transfer from phonons with a shorter MFP which increases the average MFP. As a result, $\mathrm{Si}_{0.5} \mathrm{Ge}_{0.5}$ has $>50 \%$ of heat conducted by phonons with MFP $>1 \mu \mathrm{m}$ as shown in Figure 2.5 [68]. The benefit of alloying Ge with Si can be seen by comparing the phonon density of states (DOS), as shown in Figure $\mathbf{2 . 6}$ by Weber using an adiabatic bond charge model. The individual peaks are labeled as either (T)ransverse or (L)ongitudinal and either (A)coustic or $(\mathrm{O})$ ptic [69]. The DOS for Si and Ge do not harmonize well and therefore phonons have a higher probability of scattering at Si-Ge interfaces. While point defects from alloying significantly disrupt short wavelength phonons (less than $\sim 2 \mathrm{~nm}$ ), nano-sized grains are more effective at scattering mid- to long- wavelength phonons [21]. Therefore, both alloying with Ge and reducing grain size are important for lowering the total thermal conductivity of Sibased thermoelectric materials.

While thermoelectric research has continually been performance driven, the scientific perspective also provides tremendous motivation. The $\mathrm{Si}-\mathrm{Ge}$ system is often chosen for fundamental understanding because of the huge knowledgebase with which to compare results, enabling fewer variables in the analysis and therefore more useful conclusions. The simple atomic structure and extensive semiconductor literature make Si-Ge a good stepping-stone to other more complex systems. 


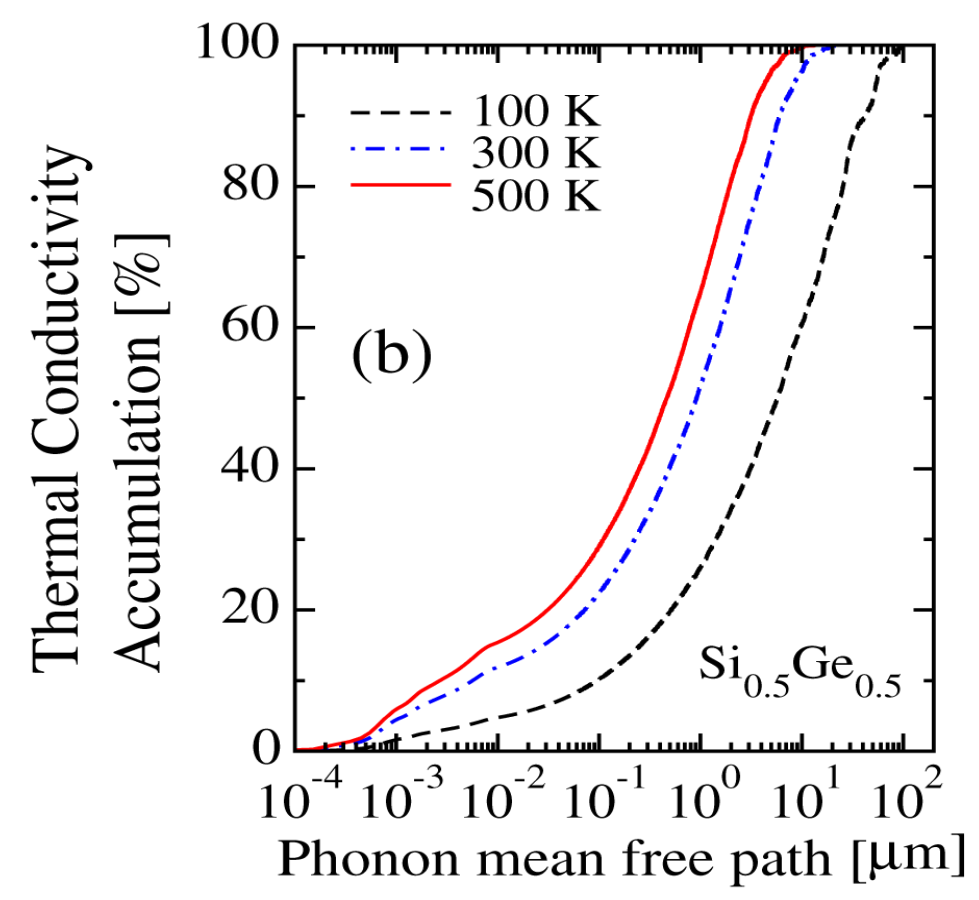

Figure 2.5. $\mathrm{Si}_{0.5} \mathrm{Ge}_{0.5}$ thermal conductivity accumulation vs. phonon MFP [68].

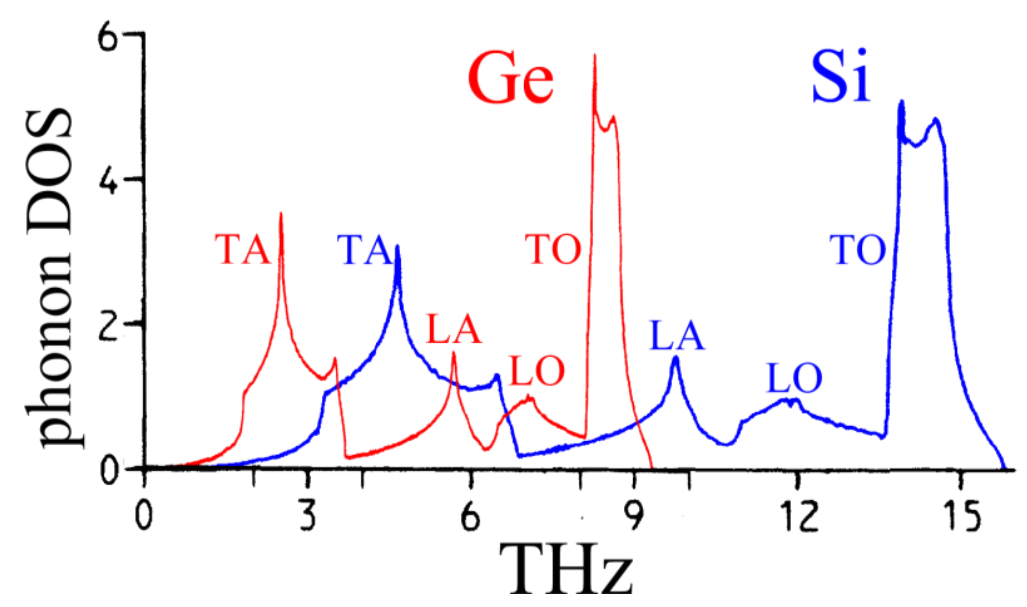

Figure 2.6. Si and Ge phonon density of states [69].

Rather than using a nanograined Si-Ge alloy to achieve low thermal conductivity, an alternative approach consists of having a multi-phase system with the $2^{\text {nd }}$ phase evenly distributed at the nanoscale. Mingo et al. theorized thermal enhancement of Si-Ge alloy by creating a "nanoparticle in alloy", whereby the insertion of $\sim 1$ vol\% of Ge or silicide nanoparticles in a $\mathrm{Si}_{0.5} \mathrm{Ge}_{0.5}$ matrix can provide a minimum thermal conductivity of $\sim 1.6 \mathrm{~W} / \mathrm{m} / \mathrm{K}$. The reduced thermal conductivity results from improved scattering of long wavelength phonons, 
given the nanoparticles are well-dispersed and sized 2-10 nm [70]. Similarly, Haskins et al. suggest that using quantum dot superlattices of Ge dispersed in a Si matrix can achieve thermal conductivity near the amorphous limit $(\sim 0.9 \mathrm{~W} / \mathrm{m} / \mathrm{K})$ without the concomitant electrical degradation; even the surface roughness of the quantum dots impacts the phonon scattering due to larger Si-Ge interfacial area [71]. To be useful, however, any nanoparticle insertion must reduce thermal conductivity more than the power factor, else ZT would decrease. 


\section{Chapter 3 : Literature Review}

\subsection{Thermoelectric}

\subsubsection{Application}

Thermoelectric materials convert a thermal gradient to a voltage potential, or vice-versa. When converting electricity to a temperature gradient, they are termed Thermo Electric Coolers (TEC's), as their primary application is for cooling with heat being rejected to a coolant or heatsink. On the other hand, when converting a temperature gradient to electricity they are termed Thermo Electric Generators (TEG's). This work focuses on only TEG's.

TEG's are practically constructed of two materials, one p-type with positive Seebeck coefficient $\left(S_{p}\right)$ and one n-type with negative Seebeck coefficient $\left(S_{n}\right)$ [50]. These elements are connected electrically in series but thermally in parallel, creating one unicouple. Since each unicouple generates relatively low power, most applications require many unicouples to be connected together to form a single module, as illustrated in Figure 3.1.

Thermoelectric generators have historically been too expensive for any application other than space travel. NASA employed Si-Ge alloys for generating electricity on space probes such as Galileo and Cassini using Radioisotope Thermoelectric Generators (RTG's). One such unicouple is shown in Figure 3.2, showing the molybdenum hot side contact on top and the thermal and electrical contacts at the bottom. Note the material composition also has a stepgrading for the lower-temperature region to improve efficiency. In this application, thermoelectrics were well-suited to providing the on-board power because of the extreme mechanical and electrical reliability. This reliability owed to having no moving parts or complex dependencies. 


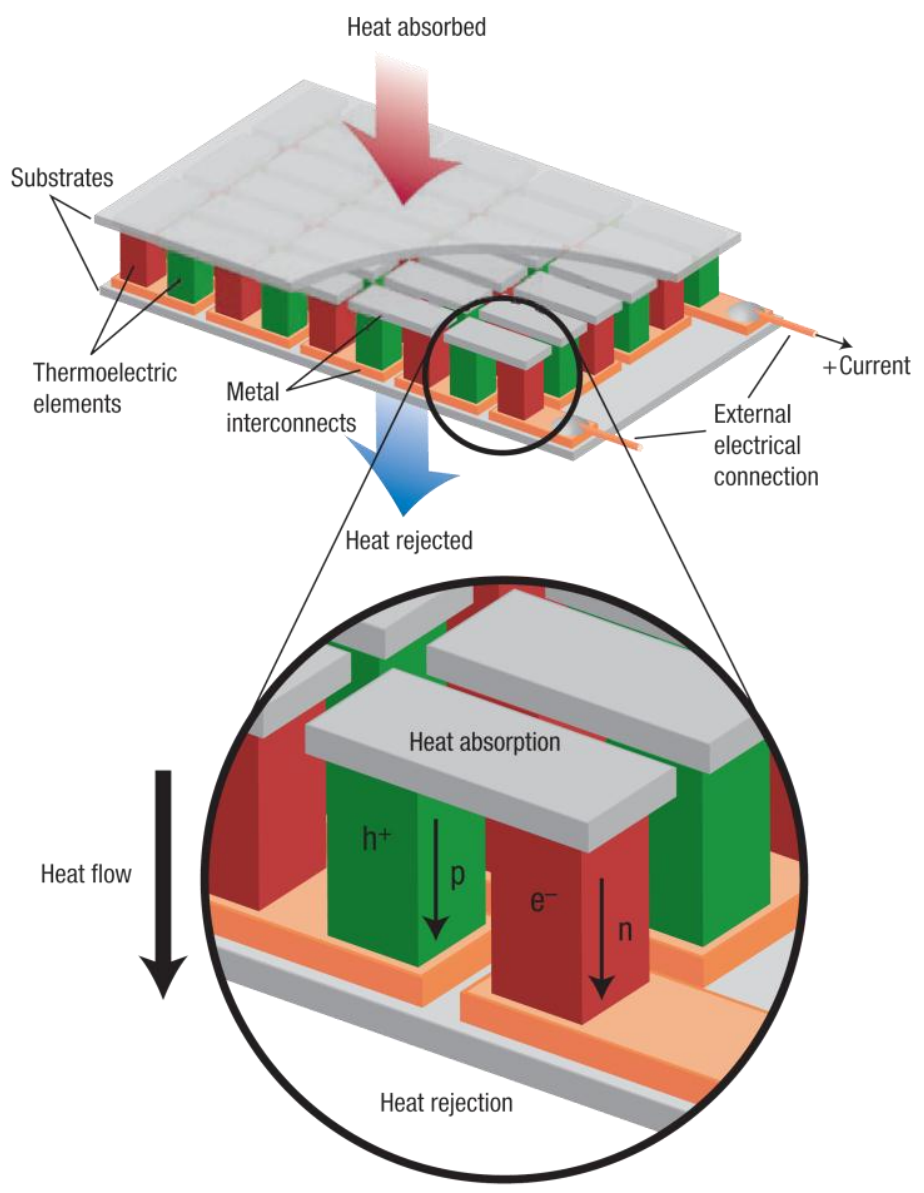

Figure 3.1. Thermoelectric module diagram [19].

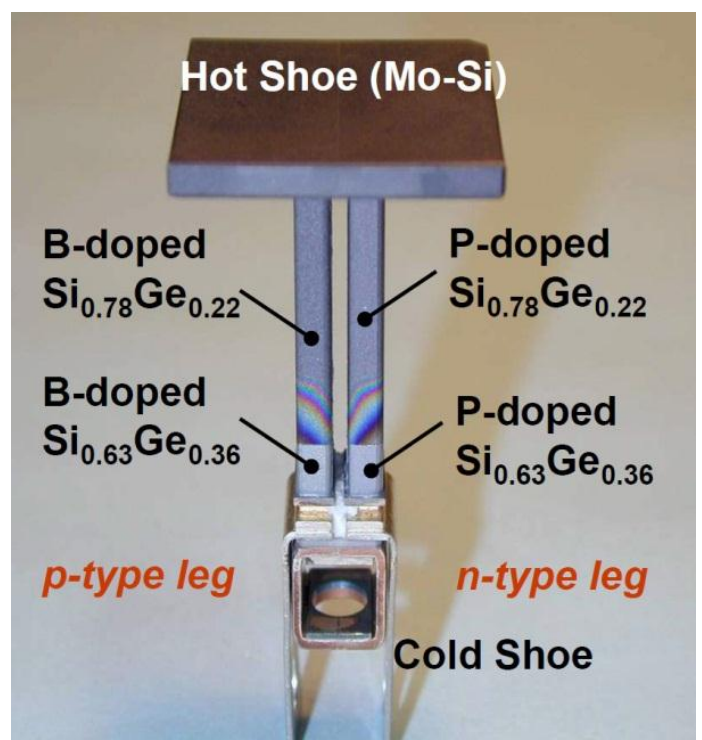

Figure 3.2. Si-Ge TEG unicouple [72]. 
Over the past few decades, automotive manufacturers have demonstrated thermoelectric generators (TEG's), but they weren't commercialized due to their high cost. Commercially, TE modules have been used for seat heating and cooling [73], but not yet automotive power generation. Recently, the U.S. DOE has been promoting thermoelectric generation in the automotive environment, listing the numerous integration benefits in terms of both comfort and up to $10 \%$ less fuel consumption [73]. In current DOE-sponsored research by BSST, hot side temperatures up to $500{ }^{\circ} \mathrm{C}$ are used (from $\sim 600{ }^{\circ} \mathrm{C}$ exhaust gas), with a cold side liquid-cooled to $<100{ }^{\circ} \mathrm{C}$; simulations show a peak efficiency $>8 \%$ and an average power $>100 \mathrm{~W}_{\mathrm{e}}$ using the FTP-75 drive cycle [3]. The first automotive TEG's could be employed commercially within a few years, with a development model shown in Figure 3.3. Other studies suggest that industrial waste heat recovery becomes reasonable for a ZT of 2 [73]. Any significant gains in the module efficiency or reductions in cost would speed the commercialization period.

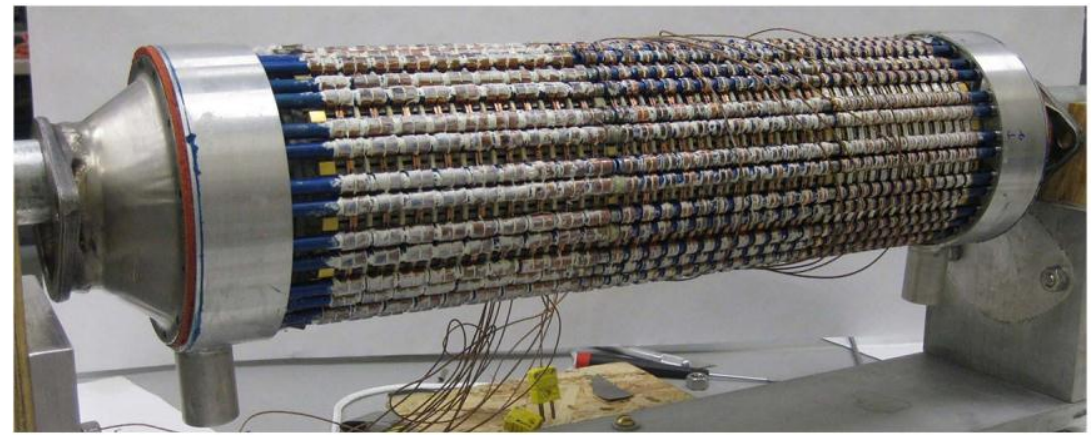

Figure 3.3. TEG from Amerigon: $500 \mathrm{~W}_{\mathrm{e}}$ from $40 \mathrm{~g} / \mathrm{s}-600{ }^{\circ} \mathrm{C}$ exhaust gas flow; $23 \mathrm{lbs}$ [74].

There has even been a high-end wrist watch (Seiko Thermic) sold that runs solely off thermoelectric power. Undoubtedly, mobile applications requiring silent reliable power are the primary market, however the tremendous availability of waste heat from all types of power plants can be retrofitted or integrated with TEG's to further improve efficiency, given the usefulness of energy in the form of electricity.

Beyond initial cost, other hindrances to application have been the:

- relatively low module efficiency

- $\quad$ significant thickness of material needed to maintain a sufficiently small heat flux

- complexity of the cooling system used to keep a high $\Delta \mathrm{T}$ across the module

- practical need for both $\mathrm{n}$ - and $\mathrm{p}$ - type devices with similar relative current densities (i.e., good compatibility) [75]. 
As the thermal conductivity of the materials is lowered, not only will it improve ZT, but it will also allow using less material for a given heat flux, improving the practicality of thin films. Lower $\kappa$ reduces the cost of the material needed, or else reduces the cost to maintain a low temperature on the cold side. For example, research by Yazawa and Sharouri considers that when using an application temperature for automotive exhaust of 60 to $600{ }^{\circ} \mathrm{C}$, the material optimization benefits more from thermal reduction than power factor enhancement in terms of applicability $(\mathrm{kg} / \mathrm{kW})$ and value $(\$ / W)$ [27]. Significantly, silicon-germanium also can be doped to make both $\mathrm{n}$ - and $\mathrm{p}$ - devices that are compatible with each other, although the same is not true for more complex compositions that are not elements based upon the carbon group (Group IV, now "14").

\subsubsection{Automotive power generation example}

Thermoelectric generators have already been demonstrated when connected to the exhaust pipe in automobiles, however the $\mathrm{Si}$-Ge system permits operation at higher temperatures than typically experienced in current applications behind the catalytic converter. Moving the TEG nearer to the engine block permits higher $T_{H}$, and the $T_{C}$ could even be cooled using the same coolant system already in place. Furthermore, as engines move to operation on ethanol and operate at higher compression ratios to extract greater efficiency, the exhaust gas temperatures will rise, which also will improve the compatibility with the Si-Ge TEG. A calculation of the feasibility follows.

The Si-Ge material performance significantly depends upon the hot side and cold side temperatures. Given the exhaust gas temperature can exceed $1100^{\circ} \mathrm{C}[76]$ and coolant temperature may be near $80{ }^{\circ} \mathrm{C}$, then it may be reasonable to assume the TEG $\mathrm{T}_{\mathrm{H}}$ is $827^{\circ} \mathrm{C}$ and the $\mathrm{T}_{\mathrm{C}}$ is $177^{\circ} \mathrm{C}$; this $\mathrm{T}_{\mathrm{H}}$ is currently higher than typical automotive engines, but if the current $\mathrm{Si}_{3} \mathrm{~N}_{4}$ and TiAl engine components can move from development to mass production then $\mathrm{T}_{\mathrm{H}}$ could be increased. A $\Delta \mathrm{T}$ of $(177-80 \approx) 100{ }^{\circ} \mathrm{C}$ between $\mathrm{T}_{\mathrm{C}}$ and the coolant should not require excessive coolant flow rates to maintain the cold-side temperature, and thus would not subtract too much from the system efficiency. From the state of the art nano-Si-Ge material [77,78], the metrics are close to:

$\bar{\sigma}=50,000 /\left(\mathrm{ohm}^{*} \mathrm{~m}\right), \overline{\mathrm{S}}=200 \mu \mathrm{V} / \mathrm{K}, \overline{\mathrm{T}}=700 \mathrm{~K}, \overline{\kappa_{e}}=0.7 \mathrm{~W} / \mathrm{m} / \mathrm{K}, \overline{\kappa_{p h}}=1.7 \mathrm{~W} / \mathrm{m} / \mathrm{K}$. 
Using Eq. 1-A to estimate $\overline{Z T}=\frac{(50000) *(0.0002)^{2}}{(2.4=0.7+1.7)} *(775)=0.65$

Therefore, the efficiency of the TEG with a $\overline{Z T}=0.65$ would be:

Using Eq. 1-D to estimate $\eta=\left(\frac{650}{1100}\right) *\left(\frac{\sqrt{1+0.65}-1}{\sqrt{1+0.65}+450 / 1100}\right)=(0.59) *\left(\frac{0.28}{1.69}\right)=9.9 \%$

Engine power output depends upon internal loads and vehicle speed. The loads may remain fairly constant, but rolling and air resistances are speed dependent. A reasonable estimate of wheel horsepower (WHP) required to travel at $65 \mathrm{MPH}$ is 25 . If the transmission is $90 \%$ efficient, then the engine must produce $28 \mathrm{HP}$ to push the car, which is $\sim 21 \mathrm{~kW}$. If internal loads of electronics and lighting add another $1 \mathrm{~kW}$, produced at $50 \%$ efficiency, then total engine load is $23 \mathrm{~kW}$. Assuming the engine efficiency is $30 \%$, and that $40 \%$ of the waste heat produced exits as exhaust gas, then $\sim 30 \mathrm{~kW}$ of heat is available for the TEG.

If the TEG absorbs $10 \%$ of the heat passing through the exhaust stream using an area 0.1 $\mathrm{m}(w) \times 0.1 \mathrm{~m}(l)$, then $\sim 3 \mathrm{~kW}$ enters the TEG; at $\eta=9.9 \%, \sim 300 \mathrm{~W}_{\mathrm{e}}$ is produced and $\sim 2.7 \mathrm{~kW}$ of heat exits the TEG. Given that $\kappa=2.4 \mathrm{~W} / \mathrm{m} / \mathrm{K}$, then the Si-Ge material thickness must be:

$$
t \cong \frac{\kappa * \Delta T * w * l}{\text { Heat Power }}=\frac{2.4 * 650 * 0.1 * 0.1}{(3000+2700) / 2}=0.005 \mathrm{~m}=5.5 \mathrm{~mm}
$$

Ideally, this thickness would be as thin as possible to minimize material cost, but it must be balanced by the cost of rejecting the heat on the cold side. Likewise, reducing thermal conductivity by a factor of 2 cuts the cost of material in half, yet more importantly reduces cost/watt by $\sim 70 \%$ so not only is ZT important to commercialization, but so is $\kappa$.

Worksheet for estimating TEG performance (Blue: input, Red: estimate, Purple: calculation): 


\begin{tabular}{|c|c|c|c|c|c|}
\hline Coeff.Drag & $\underline{0.35}$ & & $\Delta T$ & 650 & K \\
\hline FrontalArea & 24 & $\mathrm{ft}^{\wedge} 2$ & T_average & 775 & $\mathrm{~K}$ \\
\hline Vehicle speed & 65 & $\mathrm{mph}$ & S_average & 200 & $\mu \mathrm{V} / \mathrm{K}$ \\
\hline Wheel power & 24.8 & whp & $\rho \_$average & 0.02 & $\mathrm{~m} \Omega * \mathrm{~m}$ \\
\hline Transmission Eff. & $90 \%$ & & K_e_average & 0.70 & $\mathrm{~W} / \mathrm{m} / \mathrm{K}$ \\
\hline Power for speed & 27.6 & hp & К_ph_average & 1.7 & $\mathrm{~W} / \mathrm{m} / \mathrm{K}$ \\
\hline hp --> kW & 20.6 & $\mathrm{~kW}$ & $\kappa \_$average & 2.40 & $\mathrm{~W} / \mathrm{m} / \mathrm{K}$ \\
\hline Electrical load & 1.0 & $\mathrm{~kW}$ & ZT_average & 0.646 & \\
\hline Alternator efficiency & $50 \%$ & & Carnot efficiency & $59 \%$ & \\
\hline Engine power & 22.6 & $\mathrm{~kW}$ & TEG efficiency & $9.9 \%$ & \\
\hline Engine efficiency & $30 \%$ & & TEG output & 297 & W \\
\hline Heat to exhaust & $40 \%$ & & TEG thickness & 5.45 & $\mathrm{~mm}$ \\
\hline Exhaust power & 30.1 & $\mathrm{~kW}$ & Si/Ge volume & 54.5 & CC \\
\hline TEG width & 0.10 & $\mathrm{~m}$ & Si/Ge density & 2.97 & $\mathrm{~g} / \mathrm{cc}$ \\
\hline TEG length & 0.10 & $\mathrm{~m}$ & Power/Mass & 1.8 & $\mathrm{~W} / \mathrm{g}$ \\
\hline TEG heat absorbed & $10 \%$ & & $\mathrm{Si} / \mathrm{Ge}$ cost & 110 & \\
\hline TEG heat absorbed & 3009 & W & Processing cost & 993 & \\
\hline T_hot & 827 & ${ }^{\circ} \mathrm{C}$ & TEG cost & $\$ 1,103$ & \\
\hline T_cold & 177 & ${ }^{\circ} \mathrm{C}$ & $\$ /$ watt & 3.71 & /watt \\
\hline
\end{tabular}

\subsubsection{State of the Art}

After Goldsmid and Penn reported on the importance grain boundaries play in scattering phonons above the Debye temperature [79], much focus has been given to reducing lattice thermal conductivity by reducing the grain size. Rowe and Shukla published thermal conductivity results from $\mathrm{Si}_{.64} \mathrm{Ge}_{0.36}$ with $<5$ micron grain size in 1981 , as $\kappa=3.12 \mathrm{~W} / \mathrm{m} / \mathrm{K}$ at room temperature with a minimum of $\sim 1.45$ at $900 \mathrm{~K}$ [80].

Minnich et al. published a comparision of the figure of merit for different TE materials, including the improvements accomplished by nanostructuring [38], as shown in Figure 3.4 with a few additions ( $\mathrm{n}-\mathrm{Hf}_{0.6} \mathrm{Zr}_{0.4} \mathrm{NiSn}[9]$ and $\mathrm{PbTe}-2 \% \mathrm{Na}$ [81]). This overview shows that lower temperature materials have significantly exceeded $\mathrm{ZT}=1$, but that high temperature materials are only peaking near $\mathrm{ZT}=1$. On the other hand, since efficiency has a large temperature dependence, the efficiencies are likely still higher for the lower-ZT / higher-temperature materials. Regardless, the end application determines the temperature range of interest and the associated best material, so all temperature ranges may have practical relevance. Currently, the 
best reliable figure of merit has been published by Biswas et al. using a lead telluride system, which achieved a peak $\mathrm{ZT} \approx 2.2$ at $915 \mathrm{~K}[81]$.

Since much attention has been paid to thermal conductivity, Snyder and Toberer also published a comparison of the lattice thermal conductivity for a handful of materials, shown in Figure 3.5 [19]. The clathrate and skutterudite systems with 'rattlers', atoms with large displacement parameters to scatter vibrations, have very low thermal conductivity, whereas systems such as $\mathrm{Si}-\mathrm{Ge}$ and half-heuslers must use nano-sizing or other scattering centers to further decrease lattice conduction. In fact, nano-Si-Ge has been demonstrated to decrease the lattice contribution by $\sim 40 \%$ [78], which further reduction still possible with even smaller grain size. 


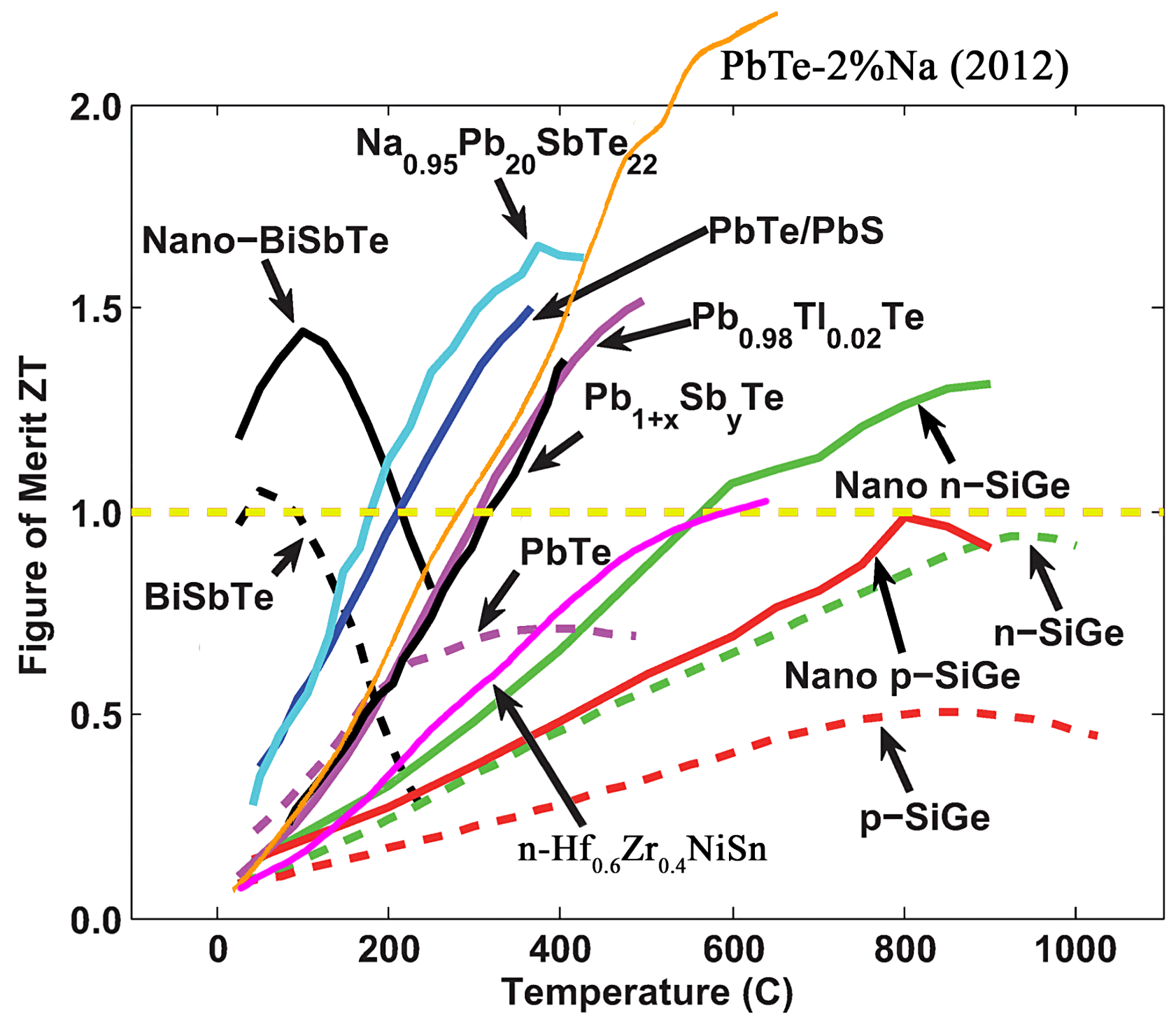

Figure 3.4. Performance of state of the art thermoelectric materials, with improvements due to nanostructuring [9], [38], [81]. 


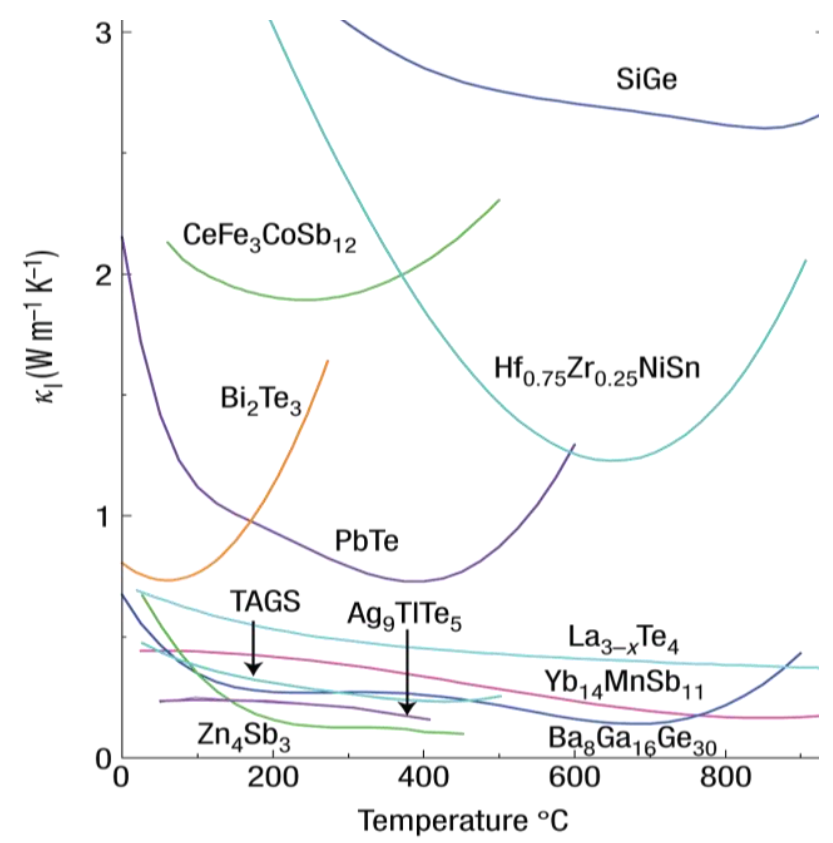

Figure 3.5. Lattice thermal conductivity of various TE material systems vs. temperature [19].

Currently the best performing $\mathrm{Si}-\mathrm{Ge}$ thermoelectric materials have been produced by joint collaboration between Massachusetts Institute of Technology and Boston College. The ntype powders were ball-milled down to an initial average crystallite size of $\sim 12 \mathrm{~nm}$ which grew to $\sim 22 \mathrm{~nm}$ after DC hot pressing, whereas the p-type powders were initially $\sim 15 \mathrm{~nm}$ yet grew to only $\sim 20 \mathrm{~nm}$. For both materials the best $\kappa_{p h}$ was $\sim 1.8 \mathrm{~W} / \mathrm{m} / \mathrm{K}$. The n-type material reached a peak ZT of 1.3 [78], and the p-type material 0.95 [77]. Figure 3.6 displays the performance of the $\mathrm{n}-\mathrm{Si}_{0.8} \mathrm{Ge}_{0.2}$ doped with $2 \%$ phosphorus and the $\mathrm{p}-\mathrm{Si}_{0.8} \mathrm{Ge}_{0.2}$ heavily doped with boron.
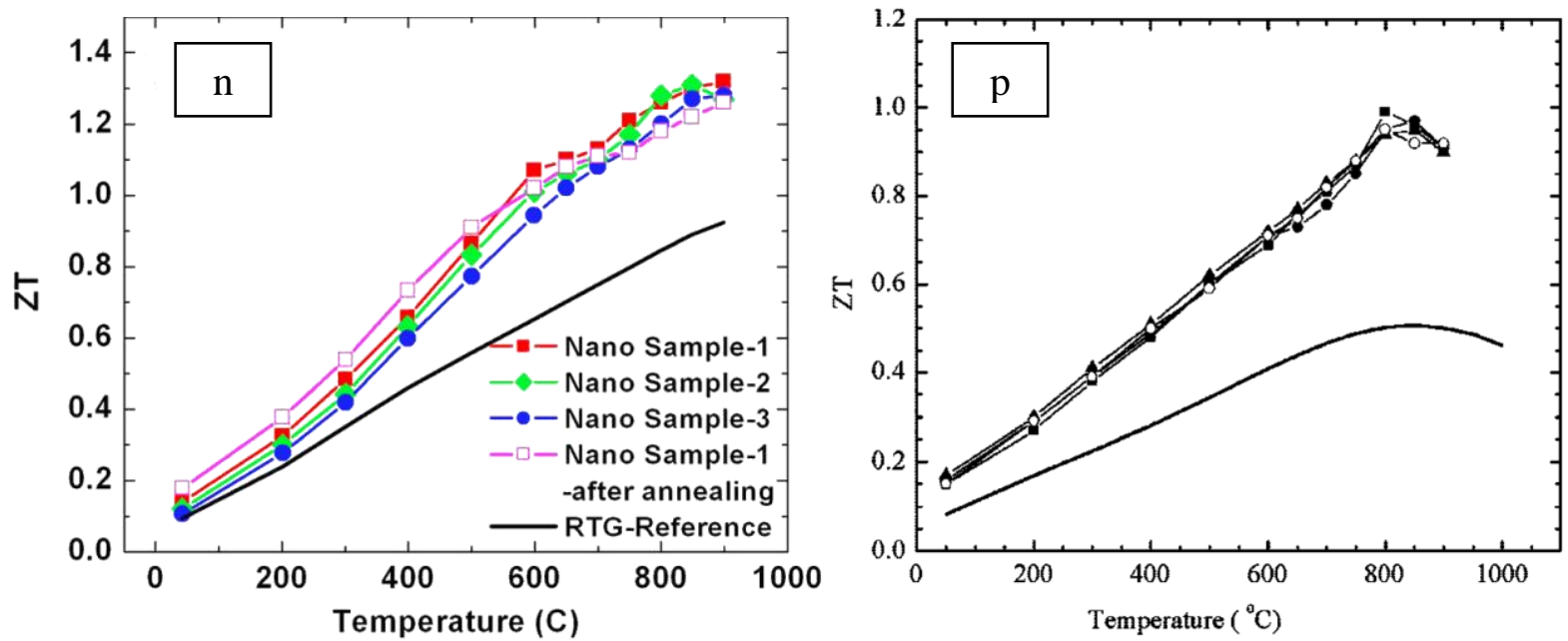

Figure 3.6. Best Si-Ge figure of merit achieved as of 2012: n [78], p [77]. 
Zamanipour et al. also achieved good results by hot pressing p-type $\mathrm{Si}_{0.8} \mathrm{Ge}_{0.2}$ at $1200{ }^{\circ} \mathrm{C}$ for 6 minutes [61]. The starting powder had an average crystallite size of $\sim 9 \mathrm{~nm}$ and after sintering the crystallite size was $\sim 25 \mathrm{~nm}$, with $\kappa_{p h} \approx 1.8 \mathrm{~W} / \mathrm{m} / \mathrm{K}$. The material reached a peak ZT of 0.8 at $800{ }^{\circ} \mathrm{C}$. Other material systems have also been advanced using nanostructuring. Poon et al. have shown a $23 \%$ performance improvement for a ZT $=0.8$ in a p-type Half-Heusler material by adding 2 vol\% nano-inclusions of zirconia before consolidation by SPS [9]. They also published the device efficiency, showing $\eta=8.7 \%$ at $\Delta \mathrm{T}=657 \mathrm{~K}$.

Adding nano-inclusions of secondary elements has been shown to improve ZT. The chosen additions should scatter phonons more strongly than charge carriers. In type-IV elements, Ge significantly reduces Si thermal conductivity, thus improving ZT. Silicon carbide could be added without affecting the doping level, however nano-inclusions of $\mathrm{SiC}$ in other materials have shown a strong reduction in electron mobility [82] so it may be a poor choice for improving Si-Ge. The very large $\mathrm{SiC}$ bandgap also likely has a large scattering cross-section for electrons.

An alternative method being investigated to improve ZT is modulation doping, using a 2 phase system with dopant precipitated but distributed as clusters at the nano-scale. This method has achieved enhanced mobility, conductivity and power factor compared to an alloy with the same overall composition, as shown in Figure 3.7. The ZT has not been improved due to significant increase in the thermal conductivity, although the ZT for the p-type material still reached 0.92 [83] and for n-type material matched the previous 1.26 [84], both at $900{ }^{\circ} \mathrm{C}$. While the figures of merit were similar, the modulation doping resulted in 2 significant changes. First, the amount of Ge used was reduced by more than half; considering that Ge costs $~ 100$ times as much as $\mathrm{Si}$, this reduction is commendable. On the other hand, the thermal conductivity increased by $>60 \%$; given that maintaining a high $\Delta \mathrm{T}$ across the TEG is critical for good efficiency yet simultaneously very difficult in terms of managing heat rejection, the material cost reduction would be overwhelmed by increased balance of systems costs. 

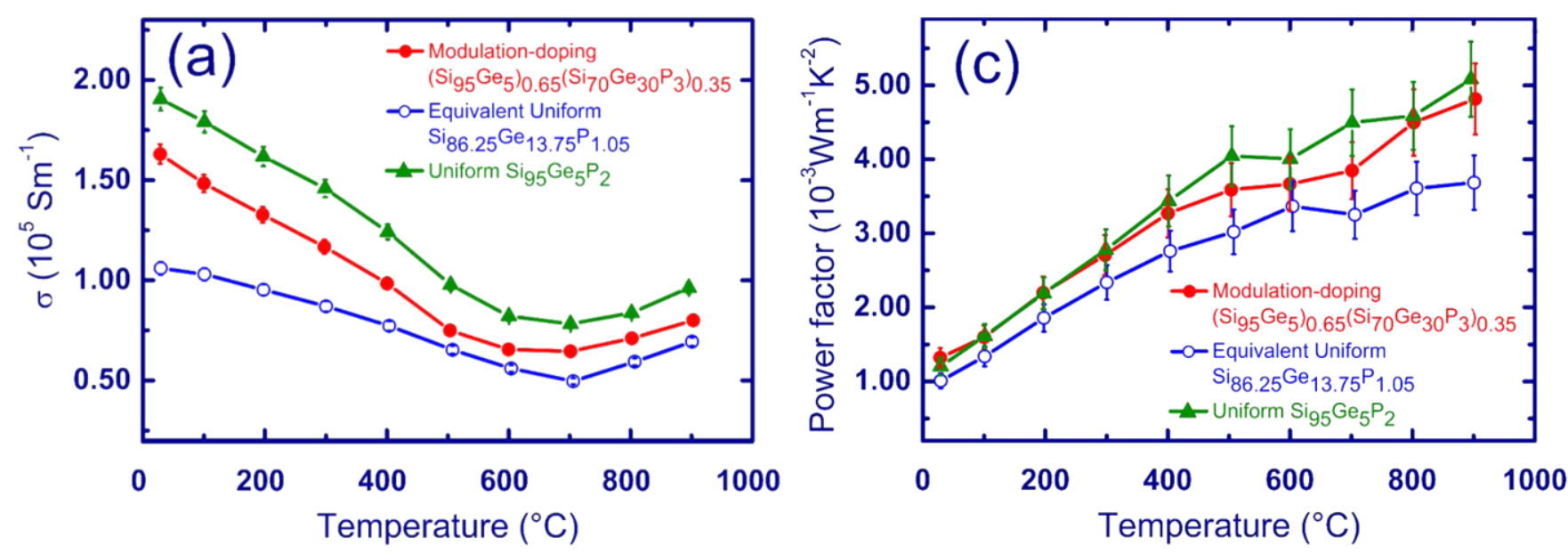

Figure 3.7. Improved electrical conductivity and power factor via modulation doping [84].

Other information has been published that incorrectly claimed extremely high peak ZT. The Si-Ge was fabricated into the n-leg of a quantum-well couple, and the authors measured a peak ZT of 3.0 at $150{ }^{\circ} \mathrm{C}$. The n-leg was composed of $\mathrm{Si}_{0.8} \mathrm{Ge}_{0.2} / \mathrm{Si}$ films, while $\mathrm{B}_{4} \mathrm{C} / \mathrm{B}_{9} \mathrm{C}$ was used for the p-leg, which together showed an efficiency of $>14 \%$ at a $\mathrm{T}_{\mathrm{H}}$ of only $250{ }^{\circ} \mathrm{C}$ [76]. After the publication of these results, it was determined that the substrate was not in fact inert, and instead had dominated some of the measurements which then invalidated the data. Unfortunately, this substrate dilution has not been publicized as well as the initial assumptions.

Overall, the state of the art has been incrementally improved over the last few decades by decreasing thermal conductivity with nano-features. With the current level of research towards finding new compositions and nanostructuring, further reduction in lattice thermal conductivity while maintaining or improving Seebeck coefficient and electrical conductivity should be feasible.

\subsubsection{Characterization Techniques}

A significant issue with thermoelectric research has been lack of accurate and complete characterization of produced samples. There is effort towards setting standards, and Oak Ridge National Laboratory (ORNL) has even distributed samples to labs across the world to determine the accuracy and repeatability of measurements [85]. The most repeatable parameter of the four curves needed for ZT calculation has been Seebeck while the most variable has been Differential 
Scanning Calorimetry (DSC), but even electrical resistivity measurements varied by up to $10 \%$. Working with films on the order of microns doesn't normally improve measurement accuracy either, however in the case of Time-Domain ThermoReflectance (TDTR) film thickness does not introduce any additional error as the penetration depth can be $<500 \mathrm{~nm}$ for Si-Ge (assuming a homogenous material).

Thermoelectrics require contact with electrically conductive substrates in practical applications, however for accurate characterization purposes the substrate must not alter the measurements, which for metal substrates may prevent electrical characterization. Likewise, thermal measurements at the macro-scale may not be reliable if the substrate makes up a large percentage of the conductance, which is especially true for thin films. The following methods are suitable for thin-film characterization of thermoelectric parameters.

Electrical measurements are made using standard methods such as two point and four point probes. To characterize the sample performance as a function of temperature, an Ulvac ZEM takes accurate measurements by measuring both the electrical resistance and the Seebeck coefficient at approximately the same time. The electrical conductivity is calculated using the sample dimensions and resistance. The ZEM uses a four point contact, with the two center probes measuring both the voltage and the temperature. This method allows accurate determination of the thermopower, as the probe thermopower is already known and can thus be subtracted from the measured voltages to get the true Seebeck coefficient. Moreover, the Seebeck coefficient and the electrical conductivity are measured at the same temperature, which ensures an accurate power factor calculation.

On bulk samples, thermal conductivity is often characterized to high temperature by using laser flash method in combination with differential scanning calorimetry. Thin films, however, must use a method which is not dominated by the substrate. Thermal conductivity may be measured by a few different options, depending upon sample properties. For bulk materials, $\kappa$ is calculated using Eq. 3-A.

$$
\kappa=D * C_{p} * \rho
$$

Eq. 3-A. Thermal conductivity measurement. 
Laser flash measures thermal diffusivity $D$, Differential Scanning Calorimetry (DSC) measures specific heat $C_{p}$, and $\rho$ can be determined by Archimedes' principal. For thin-films, thermal conductivity must be measured using alternative techniques such as: 3 omega $(\omega)$, Harman, or Time-Domain ThermoReflectance (TDTR).

To measure the overall performance of a system, the Harman technique [86] provides a relatively simple method by which conductive but electrically-isolated lines are deposited on a sample through which a pulsed current is applied [87]. Through analysis of the signal decay as a function of time, the material figure of merit can be determined. While simpler, this method does not provide scientific understanding of the individual parameters which define ZT.

A method for solely thermal characterization is called $3 \omega$ (three omega), for which sample preparation is comparable to the Harman method. This technique can be used as long as the film thickness is $>5$ times the width of metal line which is deposited [88]. An AC signal $(\omega)$

is applied to the line, and the $3^{\text {rd }}$ harmonic of the signal is used to determine how the temperature of the metal changes with time, which can then provide the sample thermal conductivity given that the heat capacity is known.

Time-Domain ThermoReflectance (TDTR) is another method for thermal characterization of thin films [89], and is the method of choice for this research. The accuracy and repeatability tends to be better than the alternatives for thin films. To use this method, a thin $(\sim 80 \mathrm{~nm})$ reflective smooth metal is deposited on the sample surface as a transducer layer. Upon impinging a laser beam on the metal, the metal transfers heat waves into the sample, and by monitoring the change of the transducer's reflectivity as a function of time, the thermal effusivity of the sample can be determine, which with the heat capacity provides the thermal conductivity.

\subsection{Sintering Technology}

\subsubsection{Sintering Mechanism}

Sintering is a method to create a solid structure from powders with or without fully melting the constituents. At temperatures near the melting point of the material, atoms within the individual particles begin significant diffusion across grain boundaries by capillary forces, forming a network of interconnected particles similar to Figure 3.8a. This initial growth is termed necking, and occurs because the lower surface area to volume ratio is more 
thermodynamically favorable [90]. With continued heat exposure, the particles begin fusing together to form larger clusters with lower surface energy. During the particle growth the porosity of the material decreases. This solid-state sintering condition is shown in the image of Figure 3.8b. Further densification creates a semi-continuous percolation network, as in Figure 3.8c. If the material is heated for sufficiently long duration or high enough temperatures, the material can reach full density with no pores or inclusions. Alternatively, it is often desired to stop processing before reaching $100 \%$ density as a means to minimize grain growth, shorten processing time, or reduce peak processing temperatures [91]. Note that each grain can still be composed of many crystallites of different orientation, which assists in phonon scattering.
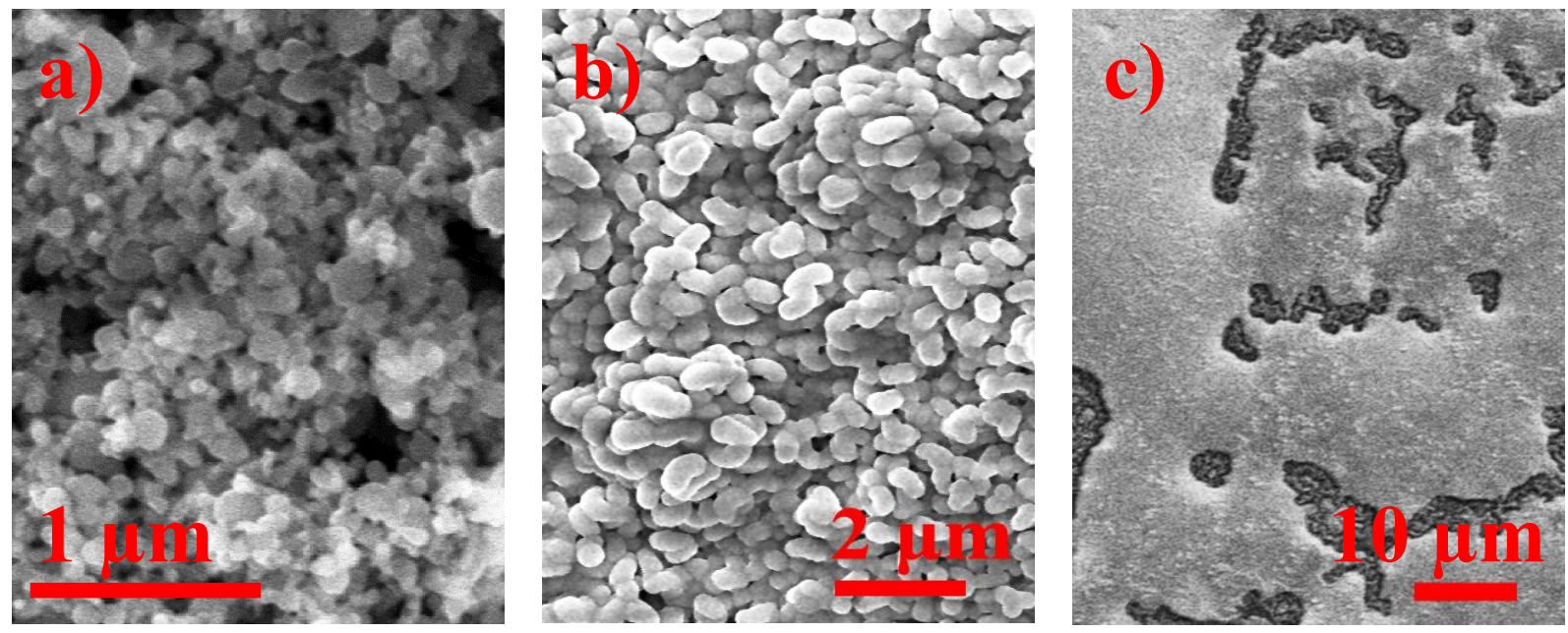

Figure 3.8. Sintering stages of Si-Ge nanoparticles from SEM:

a) necking, b) densification, c) percolation

Another type of sintering involves a multi-phase system where one constituent has a lower melting point $\mathrm{T}_{\mathrm{c}}$ than the other materials present. This liquid phase sintering involves the melting of the lower $\mathrm{T}_{\mathrm{m}}$ material such that it flows along the grain boundaries of the solid particles, thus achieving densification [92]. This method facilitates simultaneously achieving low porosity and minimal grain growth of the solid phases. When the melting points of the phases are significantly different, however, liquid phase sintering can be difficult to control with highintensity short-duration processing methods such as pulsed laser sintering. In this case, the lower $\mathrm{T}_{\mathrm{m}}$ phase tends to have a higher vapor pressure under the process conditions and thus may evaporate or ablate during laser exposure, especially if this phase absorbs laser power more efficiently than the higher $\mathrm{T}_{\mathrm{m}}$ phase. 


\subsubsection{Materials and Constraints}

Nanoparticle sintering occurs at a low temperature, as melting of $\sim 5 \mathrm{~nm}$ Si nanoparticles begins at $\sim 700{ }^{\circ} \mathrm{C}$, more than $600{ }^{\circ} \mathrm{C}$ lower than the bulk value [93]. The challenge of laser processing the extremely small particle size is avoiding ablation/evaporation, especially when the dopants are not homogenously distributed [94]. Likewise, as the particles densify very quickly, maintaining a continuous film surface can be problematic unless the area of laser impingement has a constant feed of powder material to fill the voids created during densification. If the densified layer does not wet the substrate beneath, then balling can occur [95]. Balling appears like marbles on a floor [96]. Alternatively, if the temperature is not high enough for long enough time then porosity remains in the sample. Avoiding an oxide shell on the nanoparticles is also difficult because the high surface reactivity of the silicon particles easily adsorbs residual oxygen.

\subsubsection{Pressureless Sintering and Hot Pressing}

Hot pressing of Si-Ge has been performed for many years [97], with Loughin et al. achieving ZT=0.5 in 1993 [98]. NASA used this method for sintering the Si-Ge for RTG thermoelectric production. More recently, Hwang, et al. were able to achieve a peak ZT of 0.97 for p-type $(0.5 \mathrm{wt} \% \mathrm{~B})$ using hot pressing at $1250{ }^{\circ} \mathrm{C}$ for 1 hour at $60 \mathrm{MPa}$ in 2007 [99]. The lattice thermal conductivity was $\sim 3.25 \mathrm{~W} / \mathrm{m} / \mathrm{K}$ at room temperature with a minimum near 2.65 $\mathrm{W} / \mathrm{m} / \mathrm{K}$ at $700{ }^{\circ} \mathrm{C}$. While these methods allows for high-throughput, the thermal conductivity does not compare well with more dynamic sintering methods such as laser or spark plasma sintering.

\subsubsection{Spark Plasma Sintering}

While there has been much thermoelectric research using sintering methods such as pressureless sintering or hot pressing [80], only more recently has there been literature reporting encouraging results with more dynamic processes such as spark plasma sintering (SPS or FAST) $[66,77,78,100]$. Historically, Si-Ge performance has been compared to NASA's RTG standard of ZT=0.7, having a grain size $\sim 1-10 \mu \mathrm{m}$ [100]. Currently, the best published results for Si-Ge used SPS to achieve a peak ZT of 1.3 for n-type [78] and 0.95 for p-type [77]. This fast-heating 
method retained a grain size near $20 \mathrm{~nm}$, providing significantly higher performance than past results.

\subsubsection{Laser Sintering (Annealing)}

Laser processing has been used for many types of treatments, for which Vilar gives a good overview of general usage conditions [101].

Table A - Laser process intensities and interaction times [101].

\begin{tabular}{|l|c|c|}
\hline Laser Process & $\begin{array}{c}\text { Radiance } \\
\left(\mathbf{W} / \mathbf{m m}^{2}\right)\end{array}$ & $\begin{array}{c}\text { Interaction } \\
\text { time (s) }\end{array}$ \\
\hline Laser transformation hardening & $10-10^{2}$ & $10^{-2}-1$ \\
\hline Laser melting & $10^{2}-10^{4}$ & $10^{-3}-1$ \\
\hline Laser alloying & $10^{2}-10^{4}$ & $10^{-3}-1$ \\
\hline Laser cladding & $10-10^{3}$ & $10^{-2}-1$ \\
\hline Laser CVD & $1-10^{3}$ & $10^{-1}-10^{2}$ \\
\hline Laser PVD & $10^{6}-10^{7}$ & $10^{-8}$ \\
\hline Shock hardening & $10^{7}$ & $10^{-8}$ \\
\hline
\end{tabular}

Laser sintering allows extremely fast heating and densification of nanoparticles, however it is still a novel application for thermoelectric materials. Chen et al. [95] and Bet and Kar [102] studied the pulsed laser sintering mechanism of Si nanocrystals in 2004 and 2006, respectively. Likewise, Scheller et al. [103] analyzed electrical transport in pulsed laser sintering of 100-300 $\mathrm{nm}$ undoped amorphous $\mathrm{Si}_{0.5} \mathrm{Ge}_{0.5}$ films, which showed a high concentration of dangling bonds at grain boundaries. Yet, only limited thermoelectric studies have been published using laser sintering [94,104-106]. Only one other group has presented results using laser processing on the Si-Ge thermoelectric system, achieving ZT > 0.3 at $650 \mathrm{~K}$ with a pulsed laser setup in 2011 [104]. The same group published results on pulsed laser annealing of doped Si in 2008 and SiGe in 2012, but characterization was inadequate to provide thermoelectric analysis [94], [107]. Lechner et al. using $\sim 1$ at\% doped silicon nanocrystals (Si-nc) were able to achieve resistivities and mobilities of $1 \Omega \cdot \mathrm{cm}$ and $0.5 \mathrm{~cm}^{2} / \mathrm{V} / \mathrm{s}$ for n-type and $9 \Omega \cdot \mathrm{cm}$ and $0.06 \mathrm{~cm}^{2} / \mathrm{V} / \mathrm{s}$ for p-type, at $\sim 100-150 \mathrm{~mJ} / \mathrm{cm}^{2}$ laser pulse energy density and threshold energy density of $\sim 50 \mathrm{~mJ} / \mathrm{cm}^{2}$ [94]. Sintered film thickness was $\sim 500 \mathrm{~nm}$, with morphology shown in Figure 3.9 (left). Stoib et al. 
achieved similar results with $\sim 1$ vol\% doped n-Si-Ge nanoclusters, with $\sim 300 \mathrm{~nm}$ film thickness and better resistivity of $0.15 \Omega \cdot \mathrm{cm}$ at $\sim 100 \mathrm{~mJ} / \mathrm{cm}^{2}$ pulse energy, but with more of a percolating film morphology, shown in Figure 3.9 (right). At higher temperatures (550 K), their best resistivity was $0.042 \Omega \cdot \mathrm{cm}$ for $\mathrm{n}-\mathrm{Si}_{0.8} \mathrm{Ge}_{0.2}$, and estimated carrier concentration of the films was $(4-9) * 10^{19} / \mathrm{cm}^{3}$.

Laser sintering has also been used with the $\beta-\mathrm{FeSi}_{2}$ thermoelectric material, however ZT values were not reported [105]. To the best of our knowledge, our work represents the first published results for laser sintering Si-Ge thermoelectrics with a continuous-wave diode laser [106].
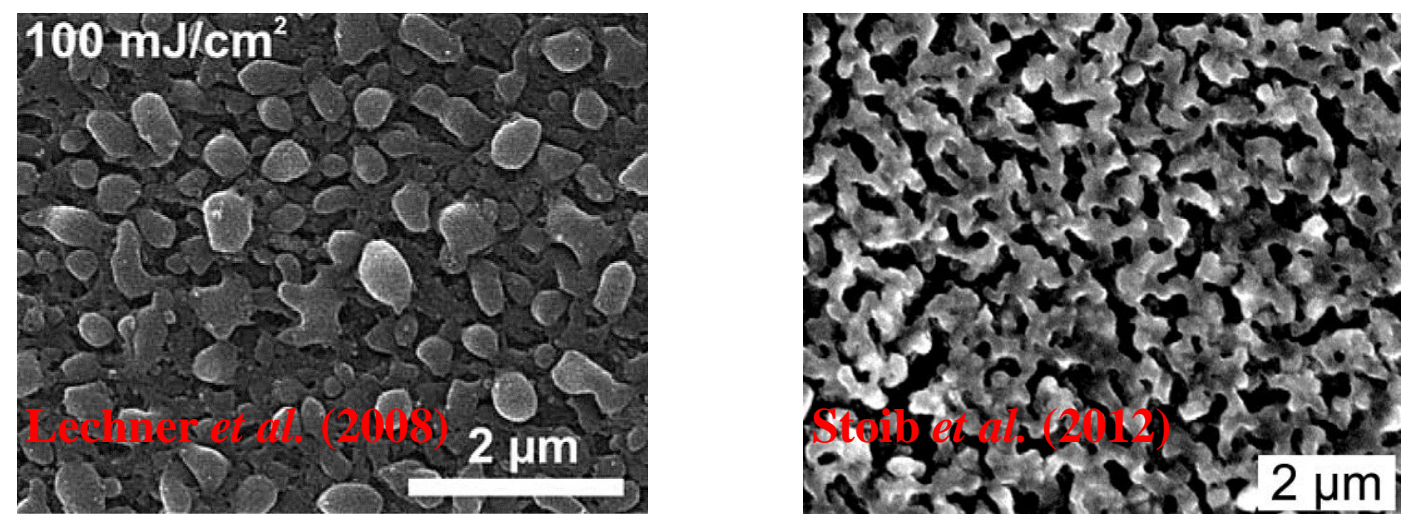

Figure 3.9. Morphology of laser sintered Si by Lechner et al. [94] and Si-Ge by Stoib et al. [107]. 


\section{Chapter 4 : Experimental}

\subsection{Materials}

\subsubsection{Powders and Dopants}

Silicon nanopowders of $50 \mathrm{~nm}$ average particle size (APS) were purchased from NanoAmor (\#0141KE, USA). The listed purity was $99.5 \mathrm{wt} \% \mathrm{Si}$, with $0.25 \mathrm{wt} \% \mathrm{Cu}, 0.13 \mathrm{wt} \%$ $\mathrm{C}$, and $0.04 \% \mathrm{Al}$ impurities. Germanium nanopowders of APS $<150 \mathrm{~nm}$ was purchased from American Elements (GE-M-021M-NP.150N, USA). Purity was listed as > 99 wt\%. Boron powder of size $<1 \mu \mathrm{m}$ was purchased from Atlantic Equipment Engineers (BO-250, USA). The listed purity was $95.64 \mathrm{wt} \% \mathrm{~B}$ with $0.31 \mathrm{wt} \% \mathrm{Mg}$.

\subsubsection{Doped Si-Ge Nanocluster Thin Films}

In a collaboration established at the 2010 Fall MRS Symposium, Prof. Kortshagen's group at the University of Minnesota (UMN) developed a novel method to deposit dense films of Si-Ge alloy using non-thermal plasma synthesis. Kortshagen has provided Gupta's group doped silicon-germanium thin films with an average particle size as small as $5 \mathrm{~nm}$ for collaborative research [48]. The nanocrystals are created from a non-thermal plasma using silane $\left(\mathrm{SiH}_{4}\right)$ and germane $\left(\mathrm{GeH}_{4}\right)$ precursors in the appropriate ratio to produce $\mathrm{Si}_{0.8} \mathrm{Ge}_{0.2}$. During fabrication, the particles are doped with either boron or phosphorus (p- or n- type, respectively), and contain very low residual oxygen. Doping level for both types was specified as 1 to 2 at $\%$ concentration, such that after laser sintering the activated dopant level would be $\sim 2 * 10^{20} / \mathrm{cm}^{3}$. UMN deposited the films on the provided substrates, over an area of $\sim 10 \mathrm{~mm}$ x $25 \mathrm{~mm}$ with thickness chosen between 2 and $45 \mu \mathrm{m}$ (results presented from $\sim 15 \mu \mathrm{m}$ thick films). Importantly, the plasmasynthesized nanocrystals were deposited in a relatively dense layer with low variation in thickness, providing an ideal sample for achieving the minimum thermal conductivity using laser sintering.

Kortshagen's group pioneered plasma synthesis of group IV nanocrystals in 2005 [108]. For $\mathrm{Si}-\mathrm{Ge}$, the precursor gases silane $\left(\mathrm{SiH}_{4}\right)$ and germane $\left(\mathrm{GeH}_{4}\right)$ are ionized by an applied electric field which accelerates electrons to temperatures high enough to dissociate the gases. Then the $\mathrm{Si}$ and $\mathrm{Ge}$ fragments come together to form nanoclusters, alloyed within the individual clusters 
[109]. The nanocluster size can be tuned from 2 to $20 \mathrm{~nm}$ [110] with a size distribution having only $\sim 20 \%$ standard deviation from the average. The nanoclusters are doped with the addition of either gaseous phosphine $\left(\mathrm{PH}_{3}\right)$ or diborane $\left(\mathrm{B}_{2} \mathrm{H}_{6}\right)$ precursors during the plasma synthesis;

Figure 4.1 shows a schematic of the plasma synthesis and particle impaction process which is able to create low-porosity films of the desired thickness on any type of substrate [110].

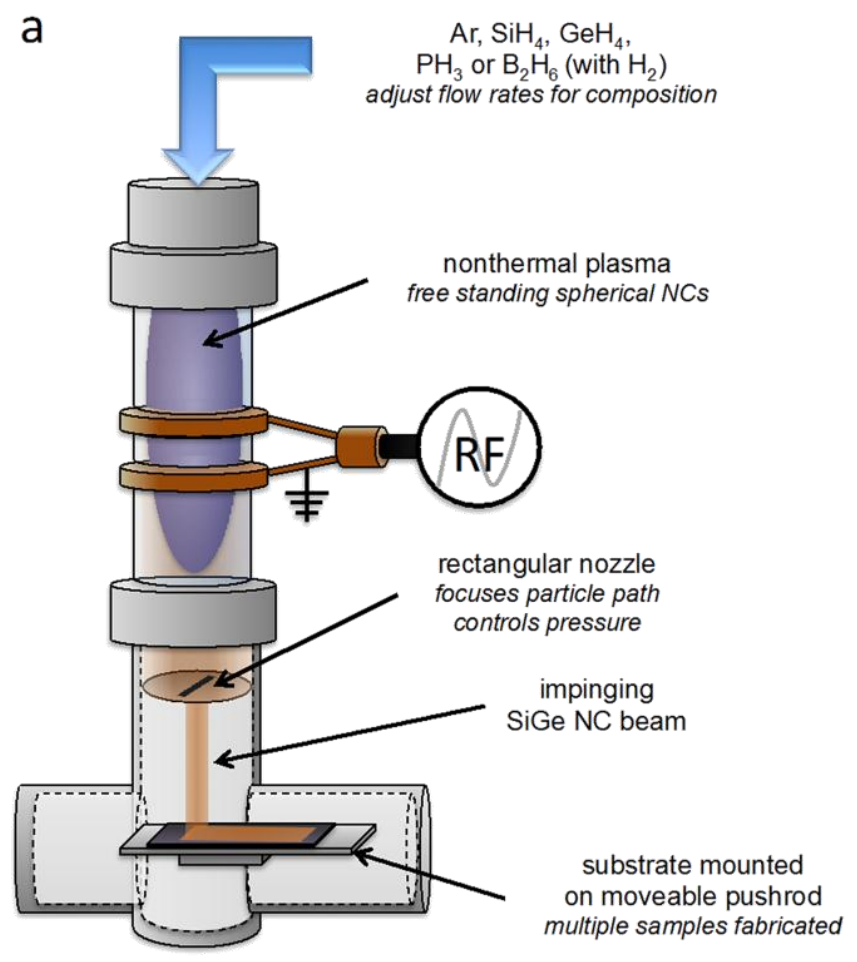

Figure 4.1. Schematic of nanocluster synthesis developed by Prof. Kortshagen's group at UMN [48].

By accelerating the nanocrystals that leave the plasma through a slit-shaped orifice, nanocrystals reach velocities $>200 \mathrm{~m} / \mathrm{s}$ and impact the substrate with sufficient force to form films with up to $\sim 50 \%$ of the solid state density [111]. This approach is highly scalable and has already been licensed to Innovalight, Inc. and Dow Corning. The films provided were produced by David Rowe (UMN) in the following fashion (note the film thickness specified is just for one example): 
1. Rinse substrates with acetone, IPA, DI water, $\mathrm{N}_{2}$ dry

2. Load in chamber

3. Ignite plasma

4. Deposit on samples 1 and 2 simultaneously for 10 minutes, until film $\sim 15$ um thick ( $\sim 150$ passes through particle beam)

5. Extinguish plasma; move samples away from nozzle

6. Purge nozzle with Ar to remove agglomerates and buildup

7. Rotate pushrod so samples 3 and 4 are facing nozzle

8. Deposit for 10 minutes on $3-4$

9. Extinguish plasma; retract pushrod into portable load-lock.

10. Bring samples, under $A r$, to $N_{2}$ purged glovebox for bagging and vacuum sealing

Nanocluster production is detailed in David Rowe's dissertation, to be published as "Impurities in Silicon Nanocrystals: The intentional and the inherent.” [112]

\subsubsection{Substrates for Powder Sintering}

Substrate selection plays a crucial role when working with thin films, thus the need to select a material which provides compatibility for a given purpose. The primary requirement is determined by whether the TE material is used for application or characterization. For application of bulk materials, the substrate should have low electrical resistivity such that it can act as a contact. For Si-Ge RTG applications, molybdenum is used as the contact on the hot side and tungsten is used on the cold side [113]. A silicide forms at the junction which enables low contact resistance and a reliable mechanical bond.

Processing of Si-Ge powders was performed on metal substrates in order to test the feasibility of laser sintering. Electrically-conductive substrates that were tested include molybdenum, tungsten, nickel, and graphite. These materials would be viable options for a multi-layer approach if the thickness could be built up to the millimeter range. Amorphous quartz was also tested as an electrically-insulating substrate.

\subsubsection{Substrates for Nanocluster Sintering}

For characterization of thin films, however, the substrate cannot be electrically conductive or else the electrical properties of the film cannot be measured, therefore ceramics must be used for the substrate to enable high temperature characterization. This restriction greatly increases the difficulty of laser sintering nanoparticles into a continuous and mechanically robust layer, as molten $\mathrm{Si}-\mathrm{Ge}$ does not easily wet ceramics while meeting the 
numerous other constraints for laser sintering, to be discussed. Substrates were chosen based upon the following properties:

A) Electrical conductivity: The first constraint requires that the substrate does not impact the film resistance by more than $1 \%$. The actual minimum resistivity of the substrate then depends upon the relative thicknesses of the film and substrate, as well as the resistivity of the sintered film. As this information is not entirely known before sintering, the substrates chosen were all high resistivity $\left(>10^{4} \mathrm{ohm}^{*} \mathrm{~cm}\right.$ at $\left.300 \mathrm{~K}\right)$ and thus should not impact electrical measurements over the entire temperature range.

B) High temperature stability: The second constraint requires that the sintered films are stably supported by a substrate to $>1000 \mathrm{~K}$. While this requirement may seem obvious as the bulk melting point of Si-Ge is well above $1000 \mathrm{~K}$, nanoparticles can have a greatly depressed melting point and pulsed laser processing is fast enough to not substantially heat the substrate. In fact, laser annealing of Si-Ge has been performed on polyimide (Kapton) substrates using such a configuration [114]. Thus, the purpose in this case is to enable CW laser sintering, where there can be significant heat build-up, and to ensure characterization to at least $1000 \mathrm{~K}$ given that Si-Ge performance typically peaks slightly above this temperature.

C) Wettability by molten Si-Ge: Wettability is important as otherwise the film may not bond well to the substrate, which can make some characterization methods impossible. Constraint A) rules out the refractory metals that would otherwise be the best choice. Instead, ceramics must be used. Jian-Guo Li investigated the wettability of molten Si on various ceramics, and discovered that the contact angle improves as the energy bandgap of the ceramic trends towards being zero, as shown in Figure 4.2 [115]. Essentially, as a ceramic becomes more metallic-like in nature, then molten $\mathrm{Si}$ is more likely to bond to it. As germanium behaves quite similarly to silicon, and Si-Ge alloys are considered to be "Si-like" when less than 85 at\% Ge [116], the molten Si data should also be applicable to Si-Ge alloys. Therefore, lower contact angles indicate easier wetting of Si-Ge, which enables using lower laser intensities to achieve an integral bond. 


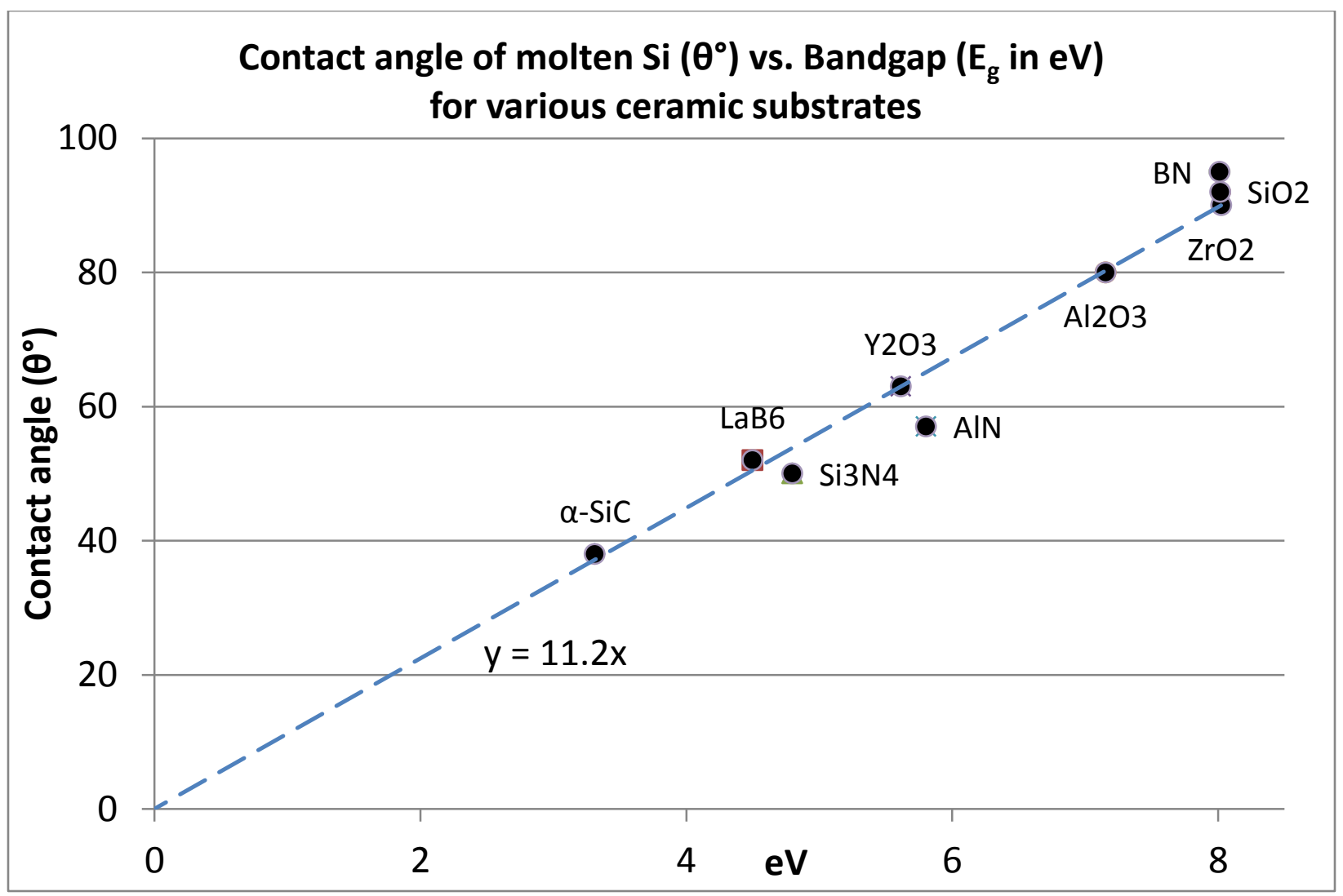

Figure 4.2. Contact angle of molten $\mathrm{Si}\left(\theta^{\circ}\right)$ vs. bandgap energy of ceramic substrates $(\mathrm{eV})$ [115].

D) Thermal shock resistance: During laser sintering, there exists a large temperature gradient at the laser spot, especially if wetting is difficult. If the ceramic substrate cannot withstand this gradient, the substrate will crack and prevent formation of continuous sintered films.

Thermal shock resistance is higher for ceramics that exhibit low thermal expansion, high fracture toughness, larger high temperature strength and potentially higher thermal conductivity [117].

E) Reactivity with Si-Ge: Reactivity with $\mathrm{Si}-\mathrm{Ge}$ cannot be extensive, or else the thin film will be mixed into the substrate or completely change phases. Slight reactivity, however, will improve wettability, such as intrinsic Si at the threshold of melting.

F) Vapor pressure at high temperature: High vapor pressure would contaminate the film during sintering. Generally, the higher the melting point of the material, the lower the vapor pressure at a given temperature. Thus, this requirement is not as applicable to ceramics. 
G) Coefficient of thermal expansion from 300 to $1500 \mathrm{~K}$ : Finally, the Coefficient of Thermal Expansion (CTE) of the substrate needs to be reasonably similar to that of the film which is being bonding, or else the film will crack upon cooling. As CTE varies with temperature, the film and substrate curves should not diverge at higher temperatures.

The materials which meet most of the above constraints include silicon carbide, silicon nitride, amorphous quartz, silicon, and aluminum nitride, although all are imperfect and properties can vary among manufacturers. The substrates chosen for laser sintering of nanoclusters include $\beta-\mathrm{SiC}, \mathrm{Si}, \alpha-\mathrm{SiO}_{2}$, and $\mathrm{AlN}$. $\mathrm{Si}_{3} \mathrm{~N}_{4}$ may also work, but would require etching of the native oxide layer before nanoparticle deposition in order to improve wettability [115].

\subsection{5 $\beta$-SiC Substrate}

The high electrical resistivity (HR) $\beta$-SiC plate obtained from CoorsTek was $2.8 \mathrm{~mm}$ thick, and substrates were diced to a size of $\sim 20 \times 11 \mathrm{~mm}$ using a water-cooled hubless diamond saw. The blade was a Disco model VT07-SD600-VC100-50. Despite the extreme hardness of $\mathrm{SiC}$ and excessive plate thickness, dicing presented no problem with a $0.5 \mathrm{~mm}$ cut depth per pass and a feedrate of $1.25 \mathrm{~mm} / \mathrm{s}$. The electrical resistivity measured $\sim 2 * 10^{5} \mathrm{ohm} * \mathrm{~cm}$ and the surface roughness $\left(\mathrm{R}_{\mathrm{a}}\right)$ measured $453 \mathrm{~nm}$ in one direction $(\mathrm{X})$ and $925 \mathrm{~nm}$ in the other $(\mathrm{Y})$.

The thermal conductivity was provided by the manufacturer, shown in Figure 4.3, with a room temperature value of $126 \mathrm{~W} / \mathrm{m} / \mathrm{K}$. 


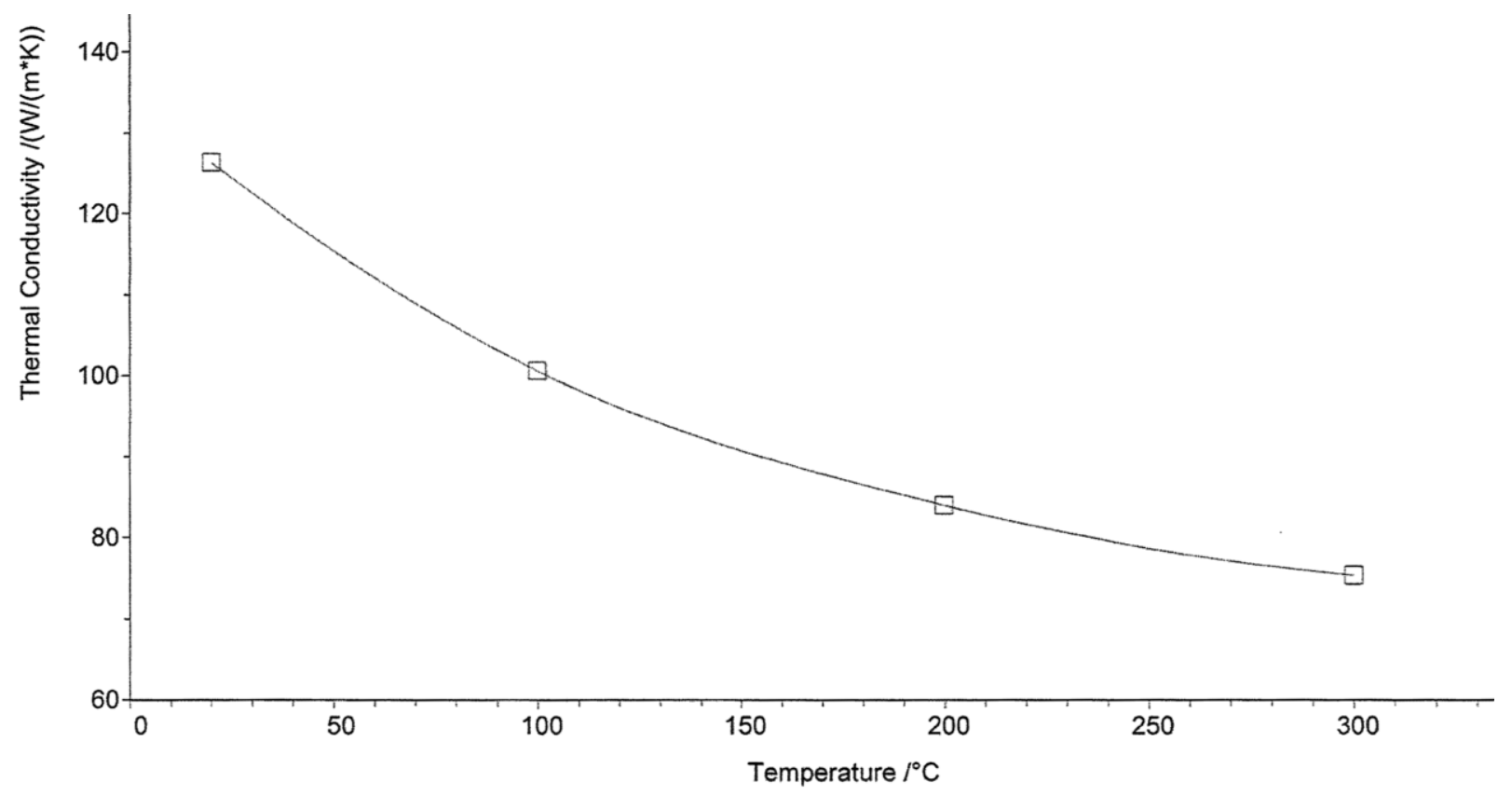

Figure 4.3. CoorsTek HR $\beta$-SiC thermal conductivity vs. temperature.

\subsubsection{Si Substrate}

The 4" $<111>$ float zone silicon wafers were purchased from Virginia Semiconductor, Inc. They were $625 \mu \mathrm{m}$ thick, double side polished, and diced to a size of $20 \times 15 \mathrm{~mm}$. The resistivity measured $\sim 6^{*} 10^{4} \mathrm{ohm} * \mathrm{~cm}$ at room temperature.

\subsubsection{AlN Substrate}

The aluminum nitride substrates were purchased from Stellar Ceramics, with a size of 57 x $57 \times 0.635 \mathrm{~mm}$. They were scribed with a diamond pen and cleaved to a reduced size of $25 \mathrm{x}$ $16.6 \mathrm{~mm}$. The polished surface was listed as having $<50 \mathrm{~nm}$ roughness, and the material specifications were listed as: Density: $3.3 \mathrm{~g} / \mathrm{cc}$, Flexural Strength: $400 \mathrm{Mpa}$, Thermal Conductivity: $170 \mathrm{~W} / \mathrm{m} / \mathrm{K}$, Specific Heat $\left(100^{\circ} \mathrm{C}\right): 780 \mathrm{~J} / \mathrm{kg} / \mathrm{K}$, CTE: $4.6^{*} 10^{-6} / \mathrm{K}$, Resistivity $(25$ $\left.{ }^{\circ} \mathrm{C}\right):>10^{14} \mathrm{ohm} * \mathrm{~cm}$.

\subsection{8 $\alpha-\mathrm{SiO}_{2}$ Substrate and Cover Plate}

The fused silica was used as cover plates for nearly all samples to reduce vapor losses during sintering and reduce contamination, but it was also used as the substrate for some samples. The substrates were purchased from Technical Glass Products, product TSC-3 made by 
Saint-Gobain with a size of $76.2 \times 25.4 \times 1 \mathrm{~mm}$. They were scribed with a diamond pen and cracked to a reduced size of approximately $25.4 \times 16 \mathrm{~mm}$. The fused silica has the following specifications: Density: $2.2 \mathrm{~g} / \mathrm{cc}$, Tensile Strength: $50 \mathrm{Mpa}$, Compressive Strength: $1100 \mathrm{Mpa}$, Thermal Conductivity: $1.38 \mathrm{~W} / \mathrm{m} / \mathrm{K}$, Specific Heat $\left(20^{\circ} \mathrm{C}\right): 750 \mathrm{~J} / \mathrm{kg} / \mathrm{K}$, CTE: $0.54 * 10^{-6} / \mathrm{K}$, Resistivity $\left(20^{\circ} \mathrm{C}\right): 10^{22} \mathrm{ohm} * \mathrm{~cm}$, Resistivity $\left(1000^{\circ} \mathrm{C}\right): 10^{10} \mathrm{ohm} * \mathrm{~cm}$, Softening Point: 1730 ${ }^{\circ} \mathrm{C}$.

\subsection{Procedures for Sample Preparation}

\subsubsection{Nanoparticle Processing}

Raw powders received minimal processing before being deposited on a substrate. The only handling was upon weighing and mixing the elements to create the proper composition. An Ohaus (USA) scale was used to measure the weight, and by using the atomic masses the desired $\mathrm{Si}_{0.8} \mathrm{Ge}_{0.2}$ ratio was achieved with $61 \mathrm{wt} \% \mathrm{Si}$ and $39 \mathrm{wt} \% \mathrm{Ge}$. For doped samples, 2 at $\%$ boron was added. Phosphorus powder was not used. To assist with dopant distribution and grain size reduction, the powders were hand mixed and crushed in a CoorsTek alumina mortar and pestle for 20 minutes. After grinding, the powders were stored in a glass tube, some in air and others in methanol. Removing the native oxide from the nanoparticles would be desirable, but filtering out the particles from hydrofluoric acid proved too difficult and unsafe.

The nanocluster (NC) films from UMN were shipped in 1" square hinged plastic sample holders, attached inside with a small piece of carbon tape to prevent the sample from bouncing around during shipment. Only a small piece $\left(\sim 0.5 \mathrm{~cm}^{2}\right)$ of carbon tape is necessary for a strong hold, and the tape is best placed near a corner to facilitate leverage during removal. While still protected in a nitrogen environment, the sample holders were then placed inside of vacuumsealed bags to reduce the likelihood of oxidation. For most samples, no pre-processing was performed on the films as instead they were directly loaded into the chamber to minimize exposure to air to $\sim 30$ seconds. Some of the vacuum bags, however, did not appear to have maintained a perfect seal.

In general, the UMN NC films had significantly lower oxygen concentration than the nanopowders, yet since the film was well-adhered to the substrate a few samples on Si substrate 
were dipped into BOE for etching. 10:1 BOE proved too strong and quickly damaged the film; a stream of bubbles was observed to be outgassing from the film, and after $\sim 15$ seconds most of the film lost adhesion. A 500:1 BOE mixture was also tested and provided better control of the etching. After 30 seconds, the sample was removed and dipped into methanol for cleaning. Unfortunately, checking the film quality in the optical microscope revealed canyons crisscrossing the film, which after processing prevented electrical continuity. Figure 4.4 shows an area which was not laser processed but was heated by the induction heater to $\sim 600{ }^{\circ} \mathrm{C}$.

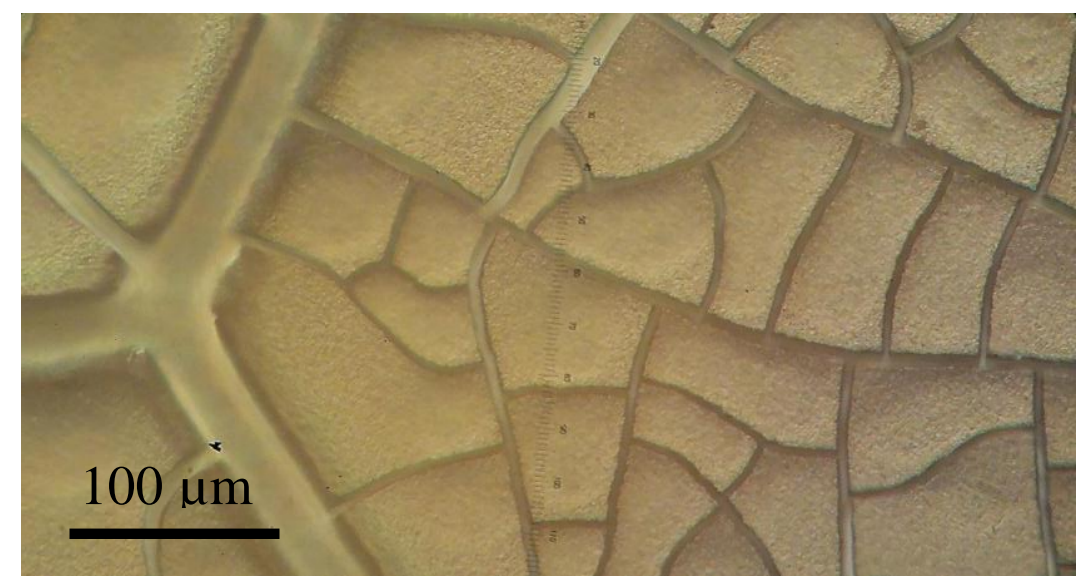

Figure 4.4. $\mathrm{Si}_{0.8} \mathrm{Ge}_{0.2}$ nanocluster thin-film after $\mathrm{BOE}$ etch and induction heating.

\subsubsection{Film Deposition Methods}

1) Powders:

a) Spread and press: mixed powders were scooped onto a substrate, spread by hand, and then flattened with a square steel slug. This method achieved good initial density and reasonable thickness consistency within individual layers, each $>\sim 50 \mu \mathrm{m}$ thick, but layer thickness was not repeatable and thin layers were not possible.

b) Spray: spray coating a film from nanoparticles in solution was difficult due to the relatively large size of the Ge and B particles, $150 \mathrm{~nm}$ and $1 \mu \mathrm{m}$, respectively. The nozzle tended to produce agglomerates which increased the starting roughness of the film.

c) Dropper: shaking the nanoparticles in liquid solution, such as methanol, would create a suspension stable for a few days. Using a dropper, the solution was applied to a 
substrate, and the solvent allowed to evaporate in air. Pulling vacuum on the chamber before the solvent finished evaporating would cause the film to crack and/or delaminate.

d) Spin coat: using a spinner to disperse the solution of nanoparticles over a substrate also failed to create consistent films, apparently due to agglomeration and substrate shape. Ultrasonication may reduce this problem.

2) Nanoclusters: $\quad$ the nanoclusters provided by UMN are described in section 4.1.2

\subsubsection{Chamber, Stage, and Induction Heater for use with $940 \mathrm{~nm}$ Laser}

Laser sintering requires scanning a beam across a sample, and due to the high temperatures involved the sample needs to be isolated from air. This experimental setup uses a two dimensional computer controlled translation stage (NLS4-4-16, Newmark Systems, USA) to move the sample up to $100 \mathrm{~mm}$ in $\mathrm{X}$ and $\mathrm{Y}$ directions. A chamber is mounted to this stage to contain the sample, and the stage can move the chamber at speeds up to $16 \mathrm{~mm} / \mathrm{s}$ (limited here by chamber inertia). The alumina silicate ceramic chamber was built to minimize the problem of oxidation during sintering by enabling a controlled atmosphere even at very high-temperatures. The chamber has gas ports for processing either in vacuum or under positive pressure from inert gas flow such as argon or nitrogen. A vacuum pump allows reaching a few millitorr of pressure; nonetheless, the chamber was not sealed well enough to completely prevent oxidation. Better results were obtained while operating with positive argon pressure. Positive pressure was implemented by continuously flowing gas into the inlet of the chamber while slightly restricting the outlet flow. Typical gas flow was $\sim 2$ CFM and pressure $<1.5$ ATM. A 3D model of the chamber was created, and shown in Figure 4.5 with side and top 2D perspectives. The model also shows the integration of the 2D translation stage, the fiber-coupled diode laser, and the induction coil. 
$\frac{\pi}{2}$
$\frac{0}{0}$
$\frac{0}{D}$
$\omega$
$\frac{0}{<}$
$\frac{1}{<}$ 


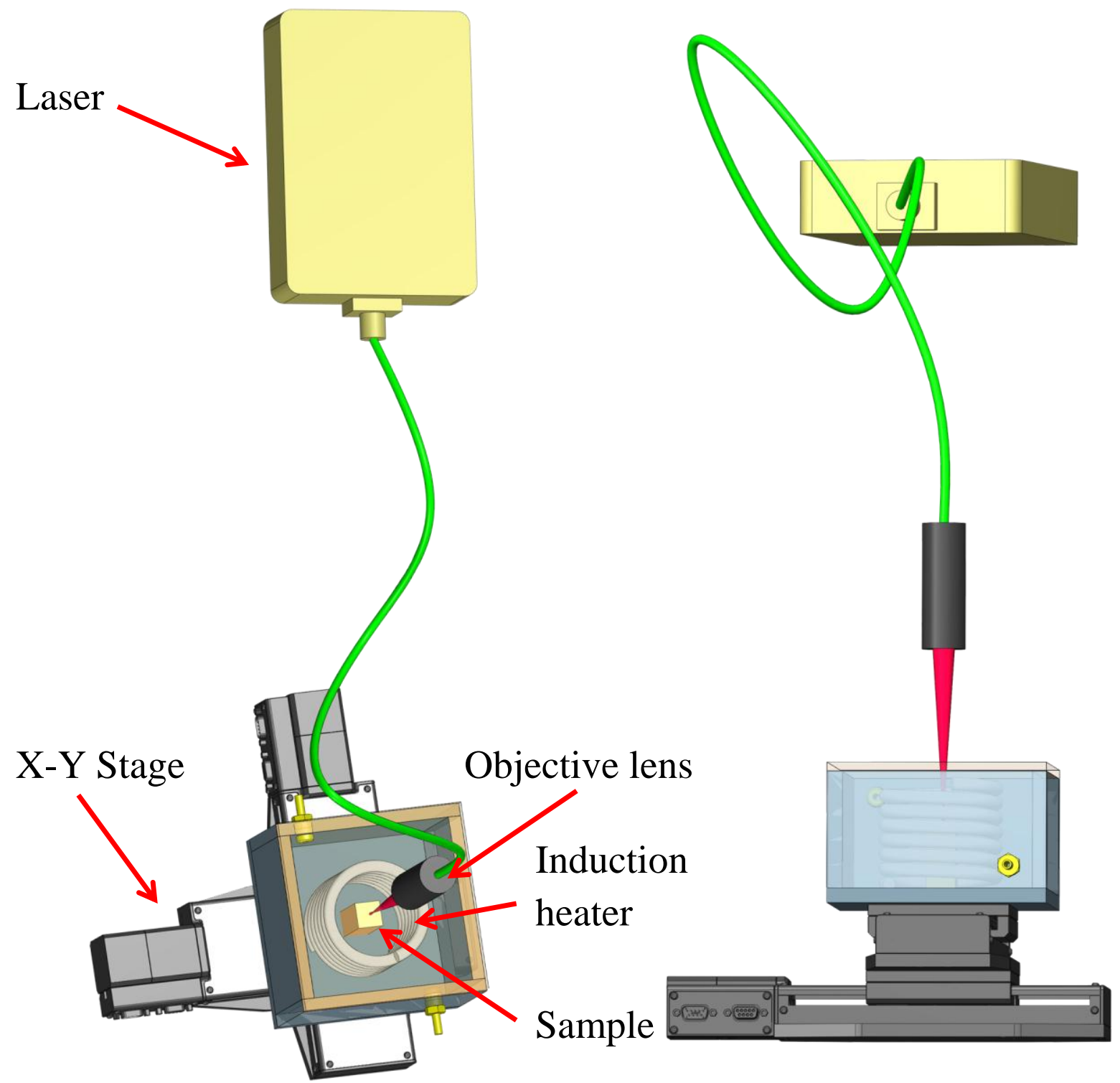

Figure 4.5. Chamber design for laser sintering (left: top and right: side).

The chamber contains a powerful induction coil to allow preheating of the sample (SP15A, MTI Corporation, USA). This coil receives power and cooling from 2 copper tubes, which are extended in a long arc to permit sufficient compliance for the stage to translate; in practice, the sample size is limited to $\sim 30$ x $30 \mathrm{~mm}$ when using the induction heater due to these copper tubes. The induction heater applies a $20-80 \mathrm{kHz}$ oscillation to the coil, which heats any material which exhibits an electric or magnetic response. This preheating reduces thermal shock during laser treatment and alleviates the formation of cracks upon laser heating and sample cooling. 
The maximum temperature achievable depends heavily on the dimensions, electrical conductivity, and emissivity of both the sample and substrate. Figure 4.6 shows induction heating of a 1 " x 1" x $1 \mathrm{~mm}$ molybdenum substrate, where the preheat temperature was $\sim 600{ }^{\circ} \mathrm{C}$. The induction heater can go to much higher temperature, but too high can negate the benefits of laser sintering and cause oxidation or dopant loss, along with changing the laser sintering conditions.

The top of the chamber consists of a 0.25 " sheet of fused quartz. This top plate rests on a silicone gasket which provides the interface to the walls of the ceramic chamber. The quartz is nearly transparent to the $940 \mathrm{~nm}$ laser wavelength, but the laser beam experiences the standard $8 \%$ total reflection loss from the change in indexes between fused silica and air. Beyond transparency, fused quartz was chosen because of its much higher thermal shock resistance than normal glass. In fact, a previous borosilicate glass cover did fracture during laser sintering. Finally, the top plate can be clamped down to allow operation at positive pressures.

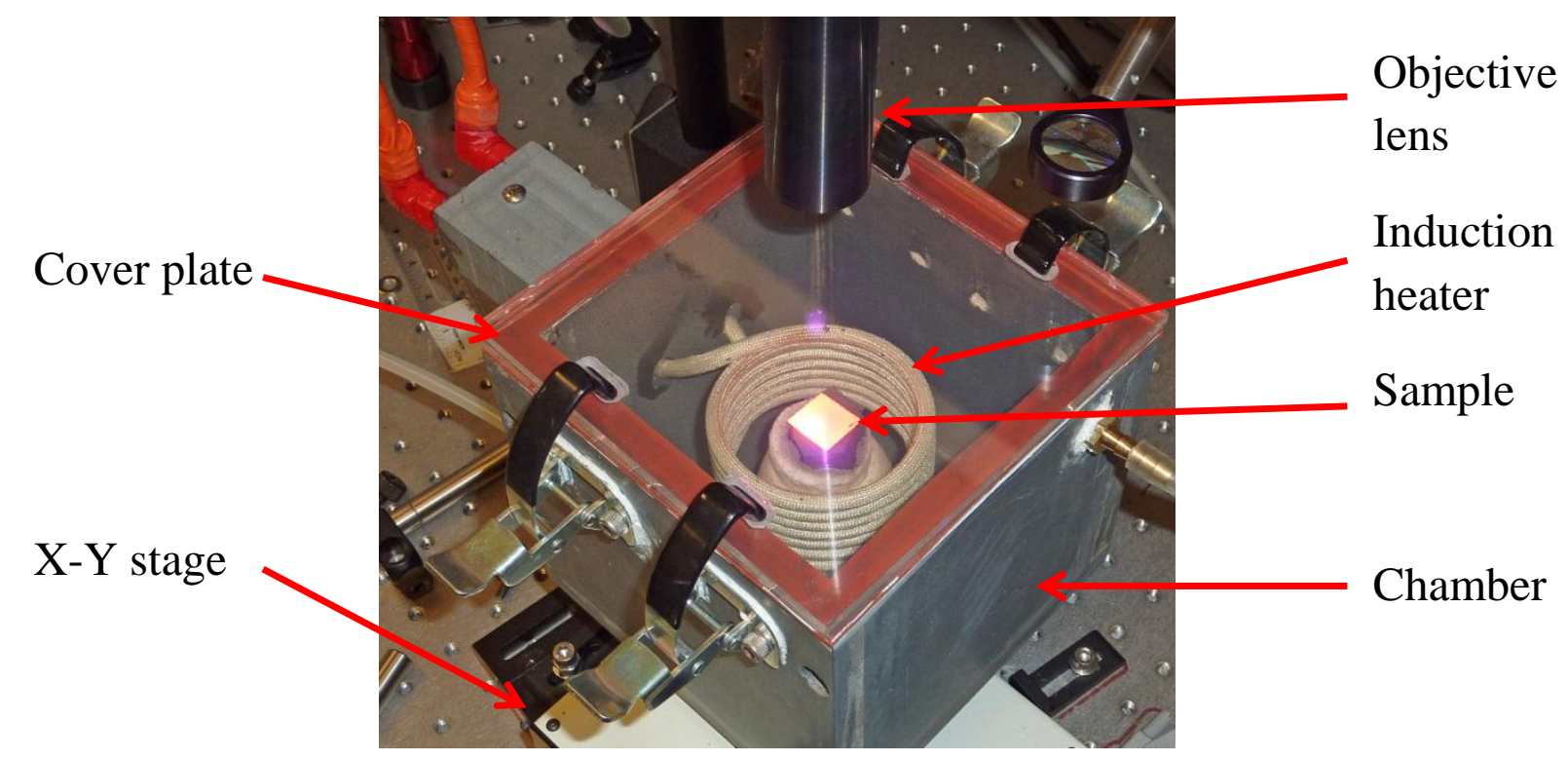

Figure 4.6. Chamber: Hybrid heating on Si-Ge on Mo substrate.

\subsubsection{Post Processing}

After sintering, the sample surface may be too rough for good electrical contact or may not have sufficient specular reflection to enable thermal characterization by Time-Domain ThermoReflectance (TDTR), thus polishing and/or lapping can be of benefit. When reduced surface roughness was beneficial, the sample was lightly polished with 2400/800 grit and then 4000/1200 sandpaper. Clean sandpaper must be used if scratches are to be avoided. For samples 
being prepared for TDTR, the surfaces were also lapped with $50 \mathrm{~nm}$ diamond compound in a cloth media.

\subsection{Laser Sintering Methods}

\subsubsection{0 nm CW Laser}

A $940 \mathrm{~nm}$ continuous wave fiber-coupled diode laser (JOLD-300-CPXF-2P2, Jenoptik, Germany) was used for sintering of both Si-Ge nanopowders and nanoclusters. The laser power applied to the sample was between 2 and 130 watts with a spot size from 0.8 to $4 \mathrm{~mm}$, but typically near the focal size of $0.8 \mathrm{~mm}$. The focal length of the objective lens was $110 \mathrm{~mm}$ and an X-Y translation stage enabled scanning the laser beam across the sample. Scan-line overlap was varied from 35 to $80 \%$ and scan speed ranged from 1 to $16 \mathrm{~mm} / \mathrm{s}$. The scan pattern employed was of zig-zag type as shown in Figure 4.7. Optionally, preheating was performed with use of the induction heater. Figure 4.7 identifies the individual components of this experimental setup. 


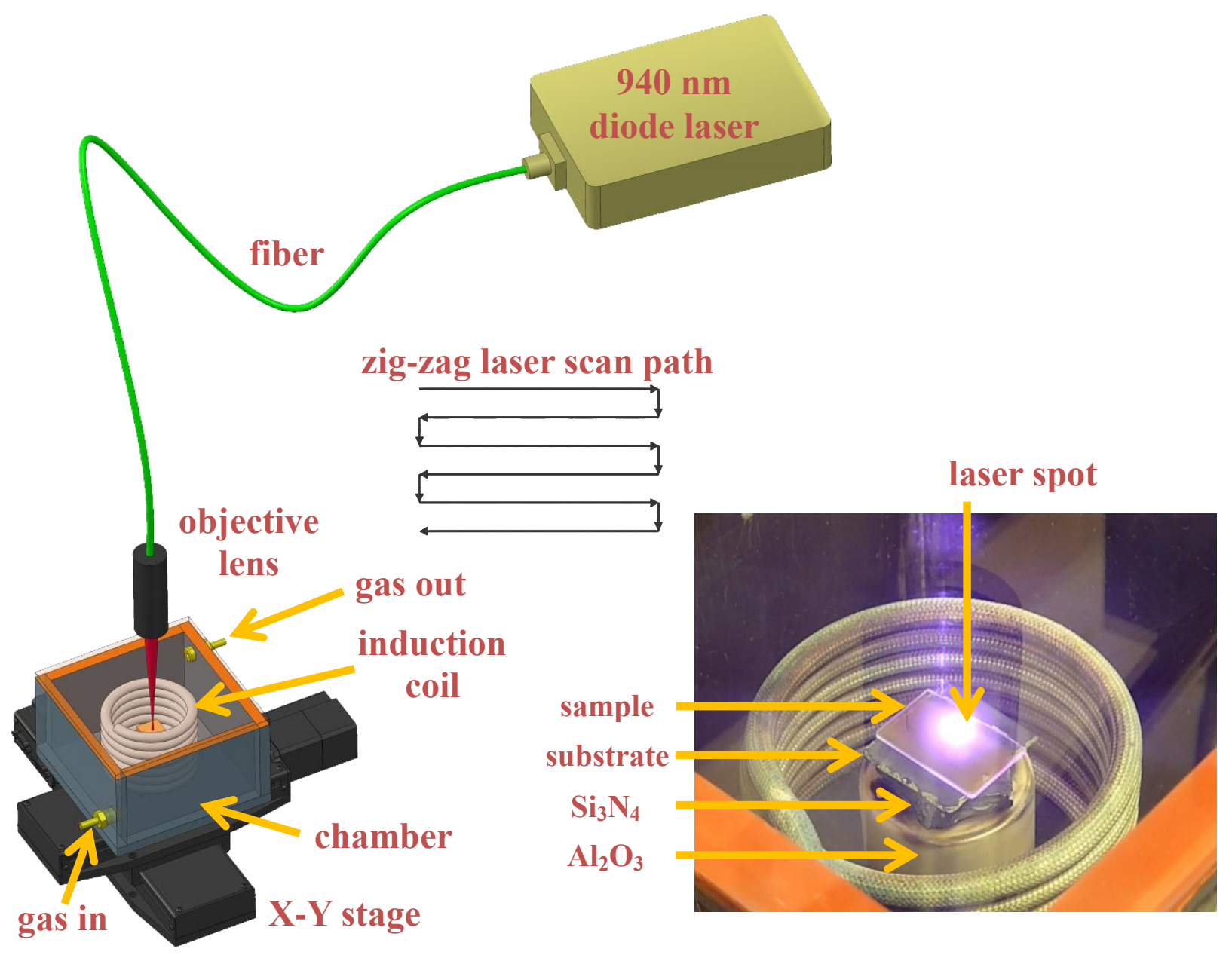

Figure 4.7. Schematic of experimental setup for $940 \mathrm{~nm}$ laser hybrid heating.

This laser is water-cooled, and for consistent power output the temperature must be allowed to stabilize first. The output power as a function of input current is shown in Figure 4.8, along with a second curve showing incident power for samples inside the chamber with a silica cover plate on top (i.e., with two sets of reflection losses). Not all samples had a cover plate, but all processing was done with the quartz chamber cover. Listed processing conditions include all reflection losses. 


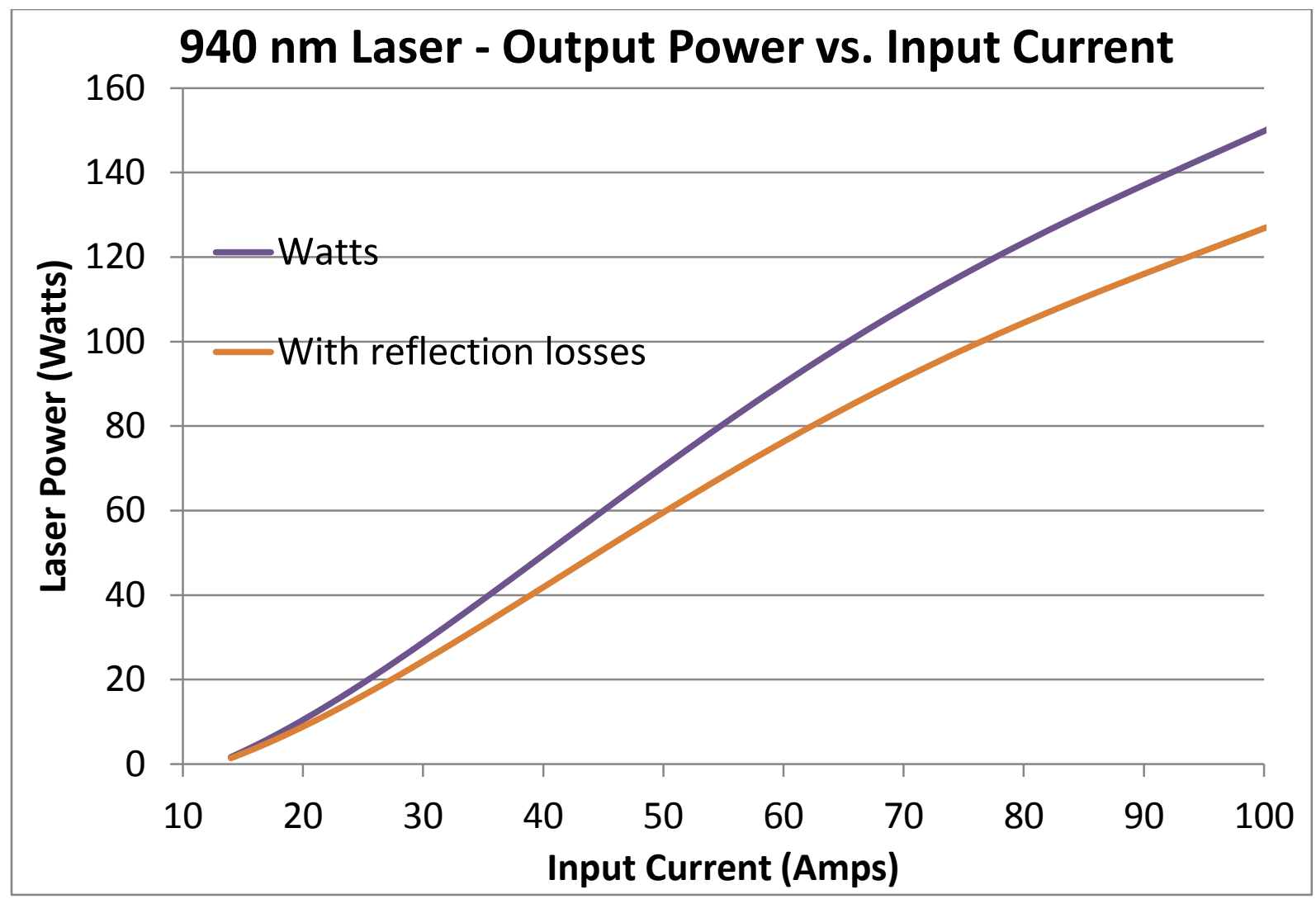

Figure 4.8. $940 \mathrm{~nm}$ laser output power vs. input current.

\subsubsection{2 nm CW Laser}

An 18 watt $532 \mathrm{~nm} \mathrm{CW}$ diode laser (Verdi-V18, Coherent, USA) was also briefly tested. This setup also used an X-Y translation stage, and a mechanical vacuum pump which was able to keep the small chamber at low vacuum.

\subsubsection{Comparison with Rapid Thermal Anneal (RTA)}

As an alternative to laser sintering, a Rapid Thermal Anneal (AS-One 100, AnnealSys, USA) was also used to anneal the films to temperatures up to $1050{ }^{\circ} \mathrm{C}$. The $30 \mathrm{~kW}$ system could quickly heat to maximum temperature, however limited flow of water cooling prevented long anneal times at temperatures over $950{ }^{\circ} \mathrm{C}$. 


\subsection{Sintering Dependencies}

\subsubsection{Films}

1) Film composition

2) Film density

3) Film roughness

4) Film thickness

5) Particle size

6) Film air exposure

7) Doping level

8) Doping homogeneity

9) Substrate material

\subsubsection{Chamber}

10) Chamber atmosphere

11) Gas flow rate

12) Sample holder

\subsubsection{Induction Preheating}

13) Pre-heating current

14) Pre-heating duration

15) Heater substrate material

16) Heater substrate position

17) Heater substrate size

18) Heater substrate shape

19) Heater coil size

\subsubsection{Laser/Stage}

20) Laser power

21) Hybrid heating

22) Cover plate

23) Laser mode

24) Laser spot size

25) Laser beam profile

26) Laser wavelength

27) Laser beam scan speed

28) Laser beam scan overlap

29) Laser beam scan pattern

30) Repetitions of scan pattern

31) Multiscan perpendicular (affects thermal conductivity, processing power)

(laser penetration, percolation)

(changes laser beam scattering)

(laser penetration, film bonding)

(thermal conductivity, depressed melting point)

(native oxide)

(electrical parameters)

(affects dopant activation, mobility modulation)

(film cracking, bonding, high-temperature stability)

(e.g., argon, nitrogen, vacuum, etc.)

(oxidation control, cooling)

(thermal delay, heat capacity)

(degree of sintering)

(outgassing and annealing)

(heat generation)

(heat generation, profile)

(heat generation)

(temperature profile)

(heat generation, profile)

(degree of sintering)

(laser power, thermal strain, grain growth)

(outgassing, contamination)

(continuous wave vs. pulsed)

(i.e., at the focal point or not)

(i.e., Gaussian or others; affects ablation and overlap)

(impacts absorption by the particles)

(densification and ablation)

(densification and continuity)

(sintered homogeneity from thermal gradients)

(affects final sintered density, grain growth)

(X-Y consistency) 


\subsection{Characterization}

\subsubsection{Morphology using SEM}

Sample microstructure was observed using Scanning Electron Microscopy (SEM) (Supra 40, Carl Zeiss, Germany). The SEM enables high resolution imaging of the surface morphology to better understand the sintering quality such as necking, grain growth, and densification. In addition, it allows verifying the width of the laser beam spot size, and when examining sample cross-sections SEM allows determination of the film thickness of unsintered or sintered layers. SEM imaging also allowed observation of any microcracks and sintered particle size.

\subsubsection{Composition using EDS}

Elemental composition was analyzed using Energy Dispersive x-ray Spectroscopy (EDS). EDS provides a quick method to qualitatively determine the atomic percentages of the constituent elements. Moreover, rather than just obtaining an average number, it allows mapping of the field of view to determine how the elements are distributed. The SEM/EDS is used to determine larger scale variations, and the TEM/EDS is used to determine nano-scale alloying of Si-Ge and the oxygen impurity distribution.

In the SEM, the EDS detector is an INCA x-sight by Oxford Instruments (England). The TEM/EDS results were partially performed at UVA and partially at ORNL. At UVA, the TEM (JEM-2000FXII, Jeol, Japan) used an EDS detector from ThermoScientific, model PGT (USA). The TEM results from ORNL (JEM-2200FS, JEOL, Japan) were provided under the HTML User program; the detector is a Bruker Quantax (USA).

EDS software generates the composition based upon the counts of the impinging radiation generated from excited electrons within the sample, which makes many assumptions about the sample conditions. One variable that can cause large inaccuracies is the presence of multiple elements with significantly different atomic masses such as exists between silicon and oxygen or silicon and germanium. In this case, to obtain accurate results a known sample must be analyzed and used to create a standard. Using that standard allows using the EDS much more accurately, here forth referred to as 'calibrated' results. Other methods of compositional analysis 
not used here include Auger Electron Spectroscopy (AES), Secondary Ion Mass Spectrometry (SIMS), and Rutherford Backscattering Spectrometry (RBS).

\subsubsection{Doping Depth}

Analysis of the dopant dilution into the Si substrates was necessary to allow calculating the electrical conductivity and power factor of the samples. Doping depth was measured for some Si substrates by combining Reactive Ion Etching (RIE) with four point probe resistance measurements (using Signatone probe with Jandel current source). The RIE parameters were:

- Pressure: 100 mTorr

- ICP power: $300 \mathrm{~W}$

- RIE power $150 \mathrm{~W}$

- $\mathrm{O}_{2}$ flowrate: $3 \mathrm{sccm}$

- $\mathrm{SF}_{6}$ flowrate: $25 \mathrm{sccm}$

The measured etch rate for the laser sintered Si-Ge material was $468 \mathrm{~nm} /$ minute, as measured by a profilometer (Dektak 8, Veeco, NY, USA). The effective conductive depth is considered the point where resistance increases by a factor of 100 from the surface measurement. Two other methods for analyzing the dopant depth which were not used include Secondary Ion Mass Spectrometry (SIMS) and Spreading Resistance Profiling (SRP). SIMS measures the atomic concentrations as a function of depth, and thus is useful for determine the total dopant concentration. SRP measures the electrical resistivity as a function of depth, and thus is useful for determining the total carrier concentration. Combining results from both methods would allow determining what percentage of the dopant is electrically active.

\subsubsection{Phases, Structure, and Stability Using X-Ray Diffraction}

X-Ray Diffraction (XRD) (XDS 2000, Scintag, CA, USA) was used for identifying phases in the nanoparticle films before and after laser sintering. These results allow determining if the $\mathrm{Si}-\mathrm{Ge}$ system is alloyed or not, and reveals if oxidation or other impurities exist. Peak broadening of diffraction peaks also permits quantitative size analysis of nano-crystals. High Temperature XRD (HT-XRD) (PANalytical X'Pert PRO, Philips, Netherlands) at ORNL/HTML was also employed to analyze how the unsintered and sintered nanocluster films changed as a function of temperature, in terms of crystallite size, lattice parameter, and phase stability. 


\subsubsection{Mobility from Hall Effect}

Hall effect measurements at ORNL/HTML using the HMS-3000 (Ecopia, South Korea) measure the carrier mobility and concentration given only the conductive thickness (thin direction) [118]. The measurements can be taken at room temperature or at $77 \mathrm{~K}$ just by adding liquid nitrogen to the cryostat. Sample mounting is shown in Figure 4.9. The hall mobility is assumed to be the carrier mobility, as with a fairly high magnetic field of $\mathrm{B}=0.54 \mathrm{~T}$ in combination with relatively heavily doped Si-Ge, the Hall factor should be $~ 1$ [119].

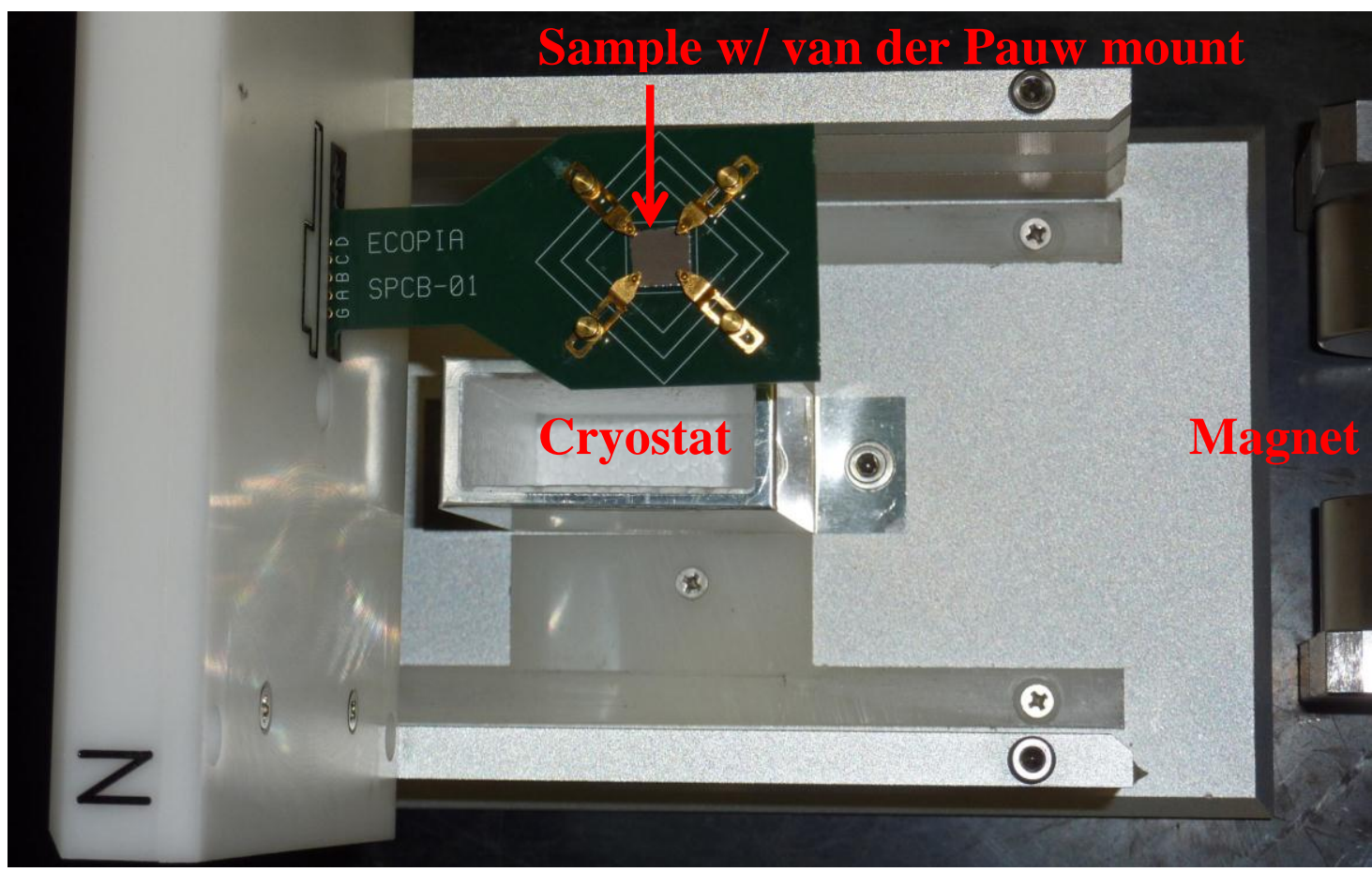

Figure 4.9. Hall mobility and carrier concentration measurement setup in Ecopia HMS-3000.

For accurate measurements of the Hall effect, NIST recommends that the sample has the proper geometry for van der Pauw contacts, as shown in Figure 4.10. Sample thickness should be relatively thin compared to the width and length, which is true for all samples measured. Samples were prepared to the ideal square shape where possible, although some samples needed to remain rectangular to permit Ulvac ZEM measurements. 


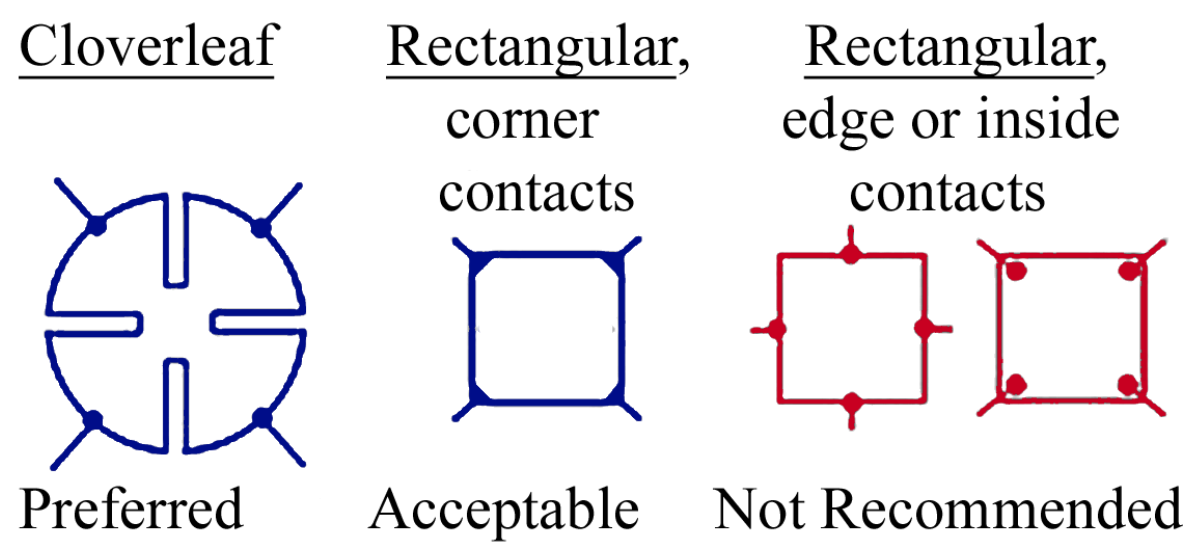

Figure 4.10. Hall effect contact geometry for the van der Pauw method.

\subsubsection{Electrical Resistivity at Room Temperature}

Electrical resistance was typically the first measurement taken on most samples. Most readings are taken using a digital multimeter (289, Fluke, USA), but a four point probe (JANDEL Engineering Ltd, UK) was also used when contact resistance made measurements difficult, or when better accuracy was needed. The resistance measurements were combined with film geometry measurements to estimate the electrical resistivity, which was used as a first order definition of successful sintering results.

\subsubsection{Thermopower and Electrical Conductivity Using Ulvac ZEM-3 vs.}

\section{Temperature}

Seebeck values (thermopower S) and electrical conductivity $(\sigma)$ are interdependent and can vary significantly as a function of temperature. For accurate analysis, both $\mathrm{S}$ and $\sigma$ should be measured on the same sample under the same conditions, which is how the Ulvac ZEM measures these parameters and why the ZEM is such a useful tool for thermoelectric characterization. Typical error is less than 4\% [85]. The ZEM model used here resides at ORNL/HTML and is a ZEM-2 that has been upgraded to a ZEM-3, with the M8 option allowing characterization from room temperature up to $800^{\circ} \mathrm{C}$. For measurements above $500{ }^{\circ} \mathrm{C}$, graphite foil was inserted between the probe tips and the sample to ensure no contamination from the R-type platinum tips, and the peak temperature was limited to $1000 \mathrm{~K}$ as an extra precaution given the high cost of replacement tips. Measurements at room temperature with and without the foil did not show any significant difference, and Dr. Hsin Wang at ORNL does not believe the foil affects results. 

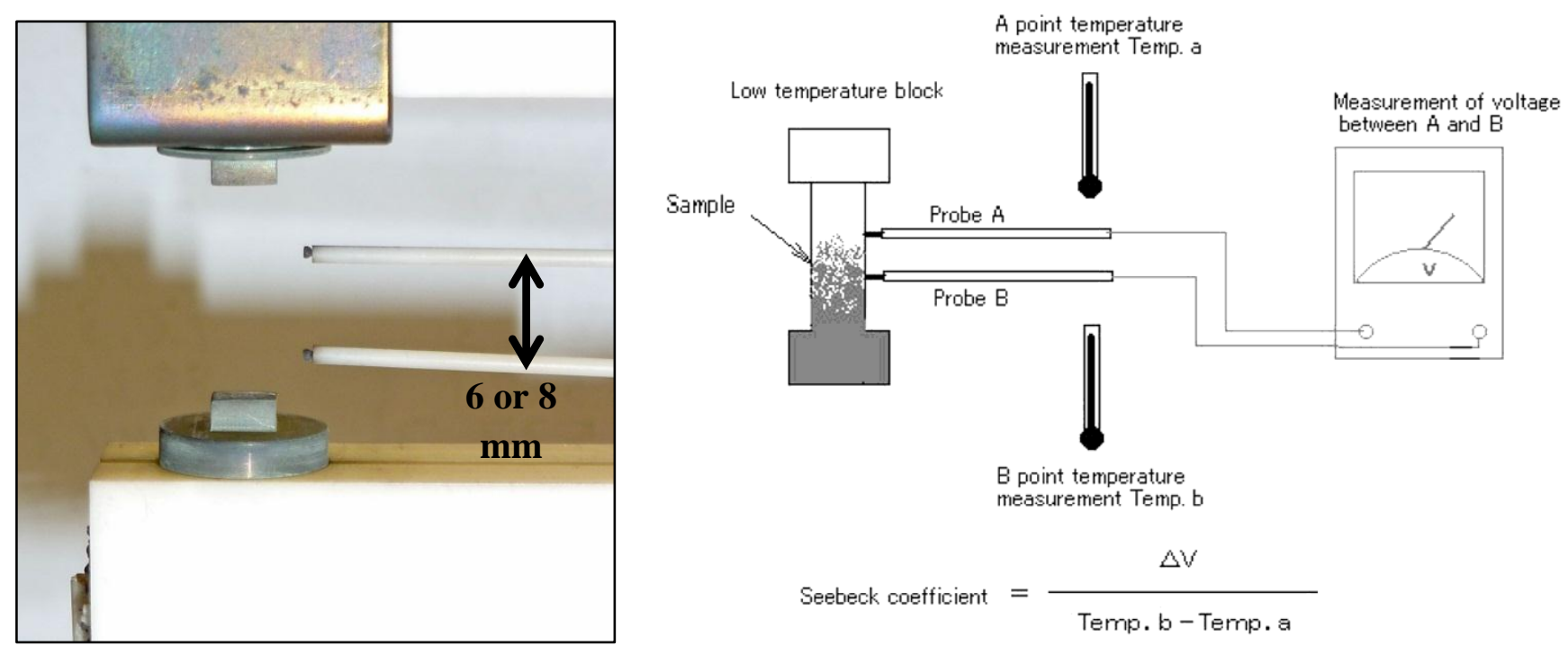

Figure 4.11. Ulvac ZEM-3 sample mounting image and schematic from manual.

The ZEM uses a four point probe setup for both the thermal and electrical measurements, as shown in Figure 4.11. Samples had to be prepared in order to fit inside the ZEM-3. Sample length needs to be $\sim 6$ to $25 \mathrm{~mm}$, width needs to be $\sim 2$ to $10 \mathrm{~mm}$, and the ends need to be parallel to provide a good electrical and thermal interface. For higher resistance samples such as thin films, it was suggested to deposit electrical contacts $\sim 5$ to $9 \mathrm{~mm}$ apart and at both ends. Contacts were created by depositing $\sim 1 \mathrm{~mm}$ wide by $\sim 200 \mathrm{~nm}$ thick metal on the samples to be measured. Initially gold had been chosen in hopes of avoiding silicide formation at high temperatures, but these samples exhibited a sharp increase in resistivity at $363{ }^{\circ} \mathrm{C}$, exactly at the eutectic point for the Au-Si system $\left(363{ }^{\circ} \mathrm{C}\right)$ [120] and Au-Ge system $\left(361{ }^{\circ} \mathrm{C}\right)$ [121]. Thus, the metal contacts used to work above $360{ }^{\circ} \mathrm{C}$ needed changing. Tungsten is suitable to $\sim 700{ }^{\circ} \mathrm{C}$ [123], but a higher temperature would be better. Beryllium would be the least-reactive, as there are no stable Be-Si compounds [122]. Alternatively, rhenium has been shown to be unreactive to $\sim 900{ }^{\circ} \mathrm{C}$ [124]. Yet, Be is considered unsafe and Re safety is unestablished and thus assumed toxic, therefore these elements were not available for deposition. A final option may be niobium, which is unreactive with Si-Ge to very high temperature [23], although it too was unavailable for deposition. Instead, silver offered safety along with reasonably low reactivity, with eutectics at $835^{\circ} \mathrm{C}$ in $\mathrm{Si}[125]$ and $651{ }^{\circ} \mathrm{C}$ in $\mathrm{Ge}[126]$; the alloy eutectic is $\sim 800{ }^{\circ} \mathrm{C}$ so it should not affect measurements. Suitable samples allowed measurement of the temperature dependence of 
thermopower and electrical conductivity, up to $800{ }^{\circ} \mathrm{C}$, the limit of the ZEM-3 M8. A schematic model of how the samples are prepared for ZEM-3 measurement is shown in Figure 4.12.

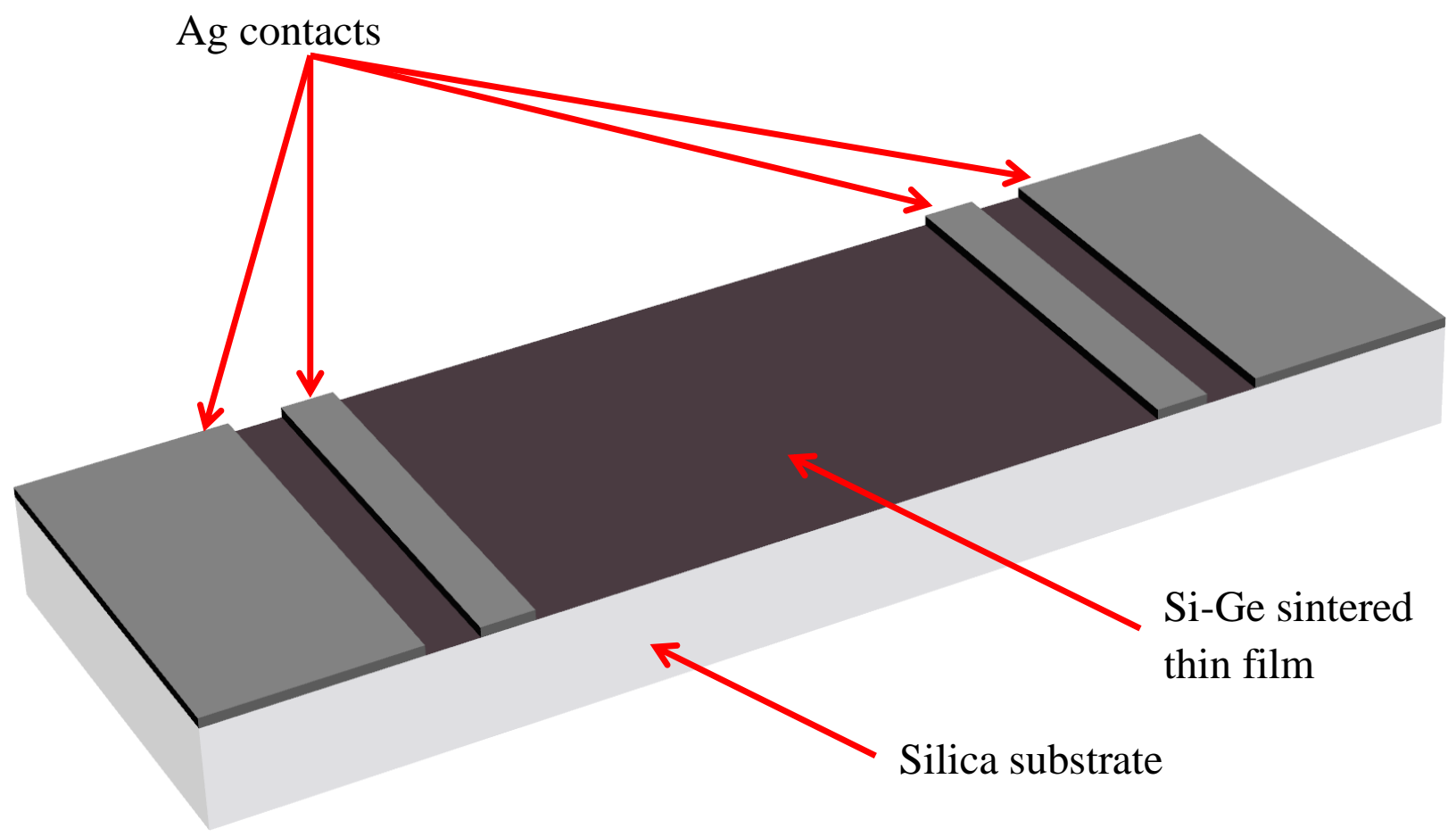

Figure 4.12. Model of Si-Ge thin film on silica substrate with Ag contacts, ready for Ulvac ZEM.

\subsubsection{Thermal Conductivity using Time-Domain Thermoreflectance vs}

\section{Temperature}

Thermal conductivity measurements are performed in collaboration with Professor Patrick Hopkins using the Time-Domain ThermoReflectance (TRTR) technique recently constructed at the University of Virginia. Hopkins' expertise with TDTR enables thermal characterization of the laser sintered thin-films. The TDTR setup uses a probe diameter of $\sim 25 \mu \mathrm{m}$ and penetration depth of $<500 \mathrm{~nm}$ for Si-Ge, given a modulation frequency of $11.39 \mathrm{MHz}$. The $800 \mathrm{~nm}$ wavelength Ti:Sapphire pulsed laser pump beam is split and frequency doubled to provide a 400 $\mathrm{nm}$ wavelength probe, which enables optical filtering of the signal to improve SNR of rough surfaces [127]. 
After all electrical measurements have been made on a sample, it can be prepared for TDTR measurements. Surface roughness must be minimal to provide a clean specular reflection, so laser sintered samples should have been lapped as a post-processing condition. For roomtemperature measurements, $\sim 80 \mathrm{~nm}$ of aluminum is deposited by electron beam evaporation as the thermal transducer [127]. For high-temperature measurements, the sample is first oxygen plasma cleaned, then a $3 \mathrm{~nm}$ barrier layer of $\mathrm{Si}_{3} \mathrm{~N}_{4}$ is deposited by plasma-enhanced chemical vapor deposition (PECVD, Plasma-Therm 7900 , USA) at $350{ }^{\circ} \mathrm{C}$ to prevent silicide formation with the transducer. Finally, $\sim 80 \mathrm{~nm}$ of platinum is deposited as the transducer. TDTR measures the thermal effusivity of the sample [128], which then allows determination of the thermal conductivity by knowing only the volumetric heat capacity $\left(C_{v}\right)$ of the material. The $C_{v}$ is calculated as a function of temperature using the long-established $\mathrm{Si}$-Ge literature values for lattice parameter and linear expansion, as discussed in sections 5.1.3 and 5.1.4. A hightemperature stage permits thermal characterization to over $1000{ }^{\circ} \mathrm{C}$. 


\section{Chapter 5 : Calculation of Si-Ge Material Properties}

To facilitate successful processing and analysis of sintered Si-Ge thin-films, a strong understanding of both the material and substrate properties is necessary. As most properties have at least some temperature dependence, this variation is provided where available. Furthermore, as thermoelectric materials are semiconductors, most electrical properties also depend on the doping level. This dependence is also provided where available.

\subsection{Thermal and General Physical Properties}

\subsubsection{Melting Point}

The melting points of $\mathrm{Si}$ and $\mathrm{Ge}$ are taken as $1685 \mathrm{~K}$ and $1210 \mathrm{~K}$, respectively. The solidus and liquidus lines of the binary $\mathrm{Si}_{(1-\mathrm{x})} \mathrm{Ge}_{\mathrm{x}}$ alloy can be approximated [129] with the following quadratic equations:

Solidus: $\quad \mathrm{T}_{\mathrm{MP} \_\mathrm{S}}=\left(1685-738 * \mathrm{x}+263^{*} \mathrm{x}^{\wedge} 2\right)$

Liquidus: $\quad \mathrm{T}_{\mathrm{MP} \_\mathrm{L}}=\left(1685-80 * \mathrm{x}-395 * \mathrm{x}^{\wedge} 2\right)$

It is assumed that the dopant has an insignificant impact on the melting point given the low concentration.

\subsubsection{Coefficient of Thermal Expansion (CTE)}

The coefficient of thermal expansion (CTE) of the constituents can be relevant if the material is not homogenous, as well as at the interface with the contacts or substrate. Figure 5.1 graphs the values compiled by Slack and Bartram [130] for Si and Ge for temperatures above liquid nitrogen $(77 \mathrm{~K})$, although very low temperature data from Smith and White is also included [131]. The data stops at $1080 \mathrm{~K}$ and $1573 \mathrm{~K}$ for Ge and $\mathrm{Si}$, respectively; thus to extrapolate the CTE of Si-Ge alloys above $1080 \mathrm{~K}$, polynomial regressions were performed from the nearly linear portion of the curves, from 400 to $1080 \mathrm{~K}$ for Ge and from $673 \mathrm{~K}$ to $1573 \mathrm{~K}$ for $\mathrm{Si}$. The solidus limit is also graphed using diamond markers at 5 atomic $\%$ intervals. 
Si-Ge alloy CTE values are estimated by linear interpolation using the atomic composition with $\mathrm{Si}_{1} \mathrm{Ge}_{0}$ and $\mathrm{Si}_{0} \mathrm{Ge}_{1}$ as the endpoints for a given temperature. Beta silicon carbide is also graphed, data taken from [132]. As shown in Figure 5.1, the coefficient of thermal expansion of $\mathrm{SiC}$ matches well with $\mathrm{Si}-\mathrm{Ge}$ alloys of low Ge concentration.

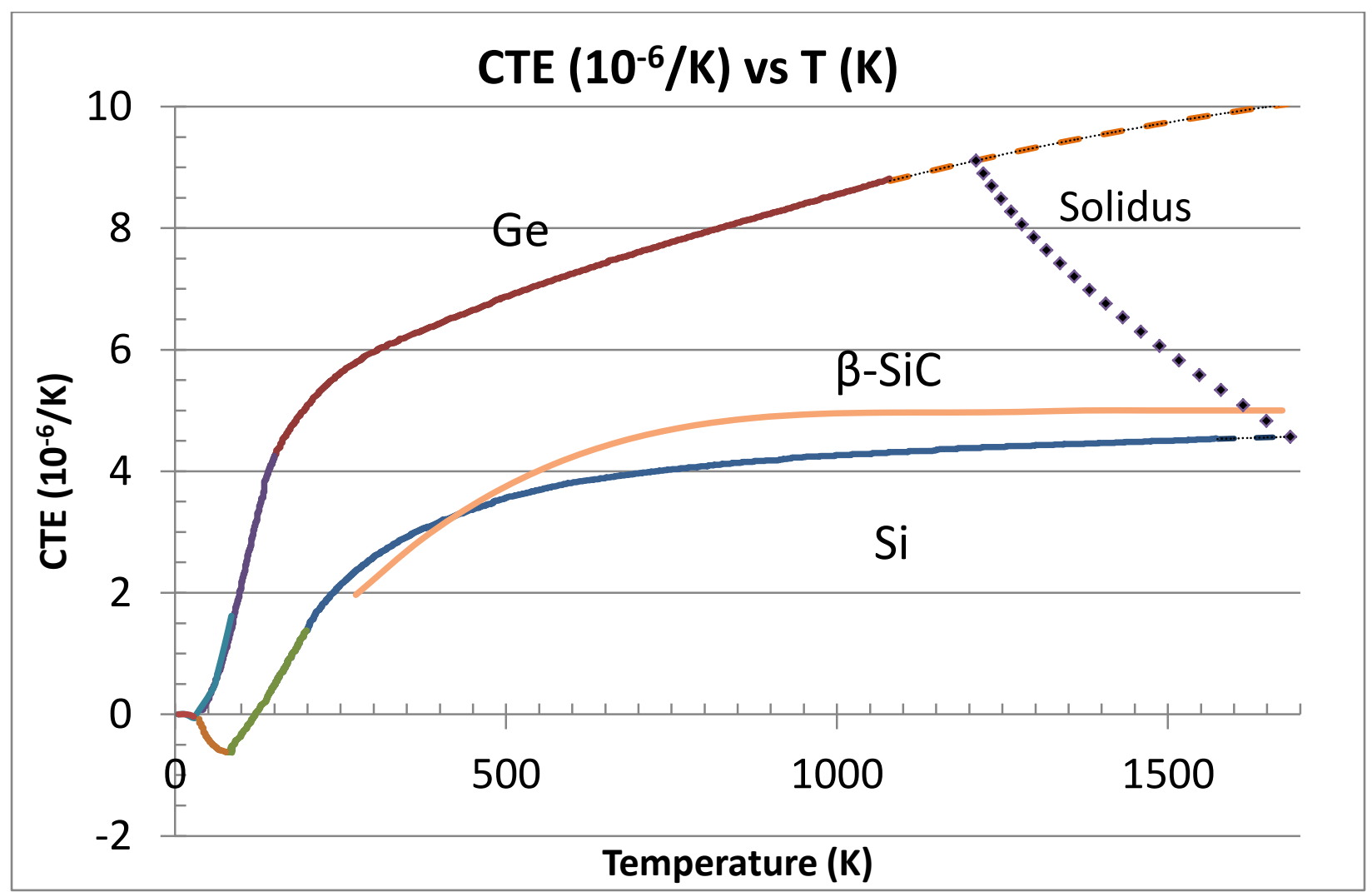

Figure 5.1. Coefficients of thermal expansion for $\mathrm{Si}, \mathrm{Ge}$ and $\beta-\mathrm{SiC}$.

\subsubsection{Lattice Parameter}

The lattice parameter of Si-Ge alloys varies slightly from the linear interpolation given by Vegard's law. A better model is provided using a quadratic fit given the Ge atomic \%:

$$
\AA=0.02818271 \times(\% G e)^{2}+0.1981446 \times(\% G e)+5.431489
$$

Eq. 5-A. Lattice parameter of Si-Ge at 298 K [133]. 


\subsubsection{Linear Expansion (Lattice Parameter vs. Temperature)}

The recommended linear expansion values are taken from Touloukian's work in the Thermophysical Properties of Matter, Vol. 12 [134].

$$
\begin{aligned}
G e_{\text {Low } T}=(-0.00089+ & 2.626 \times 10^{-6} \times(T-100)+1.463 \times 10^{-8} \times(T-100)^{2}- \\
& \left.2.221 \times 10^{-11} \times(T-100)^{3}\right)
\end{aligned}
$$

Eq. 5-B. Linear expansion of Ge (100-293 K) [134].

$$
\begin{gathered}
G e_{H i g h T}=\left(5.79 \times 10^{-6} \times(T-293)+1.768 \times 10^{-7} \times(T-293)^{2}-4.562 \times\right. \\
\left.10^{-13} \times(T-293)^{3}\right)
\end{gathered}
$$

Eq. 5-C. Linear expansion of Ge (293-1200 K) [134].

$$
S i_{\text {Low } T}=\left(-0.00021-4.149 \times 10^{-7} \times T-4.620 \times 10^{-10} \times T^{2}+1.482 \times 10^{-11} \times T^{3}\right)
$$

Eq. 5-D. Linear expansion of Si (20-293 K) [134].

$$
S i_{H i g h T}=\left(-0.00071+1.887 \times 10^{-6} \times T+1.934 \times 10^{-9} \times T^{2}-4.544 \times 10^{-13} \times T^{3}\right)
$$

Eq. 5-E. Linear expansion of Si (293-1600 K) [134].

For high temperature, the thermal linear expansion of Si and Ge are interpolated and then combined with Eq. 5-A to model the lattice parameter as a function of both composition and temperature. The impact of doping is assumed to be negligible. 


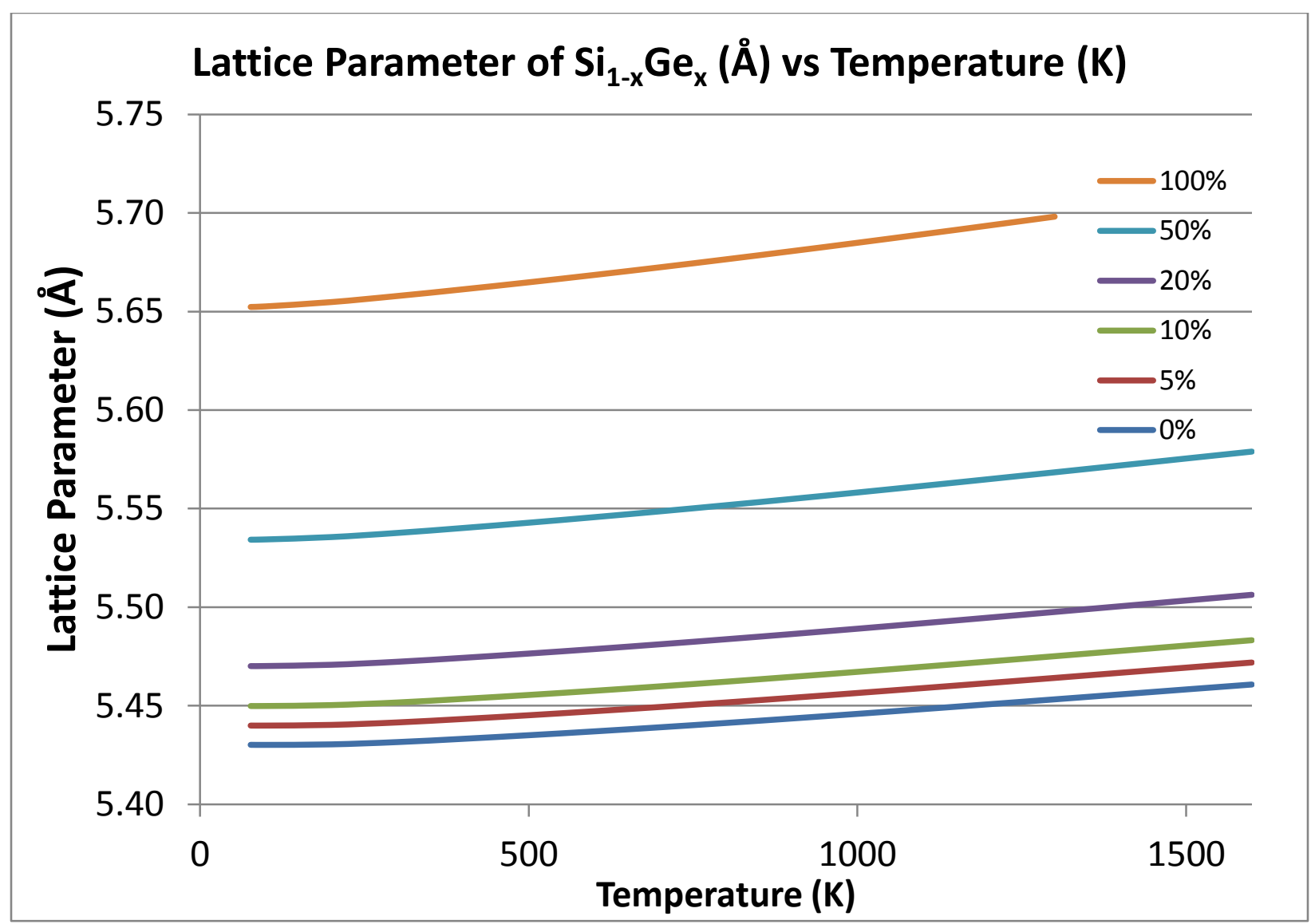

Figure 5.2. Lattice parameter of $\operatorname{Si}_{1-\mathrm{x}} \mathrm{Ge}_{\mathrm{x}}(\AA)$ vs. temperature $(\mathrm{K})$, where $\mathrm{x}$ is at\% Ge.

\subsubsection{Heat Capacity}

The anharmonic specific heat vs. temperature curves for Si and Ge were used from Kagaya et al. as depicted in Figure 5.3 [135]. 


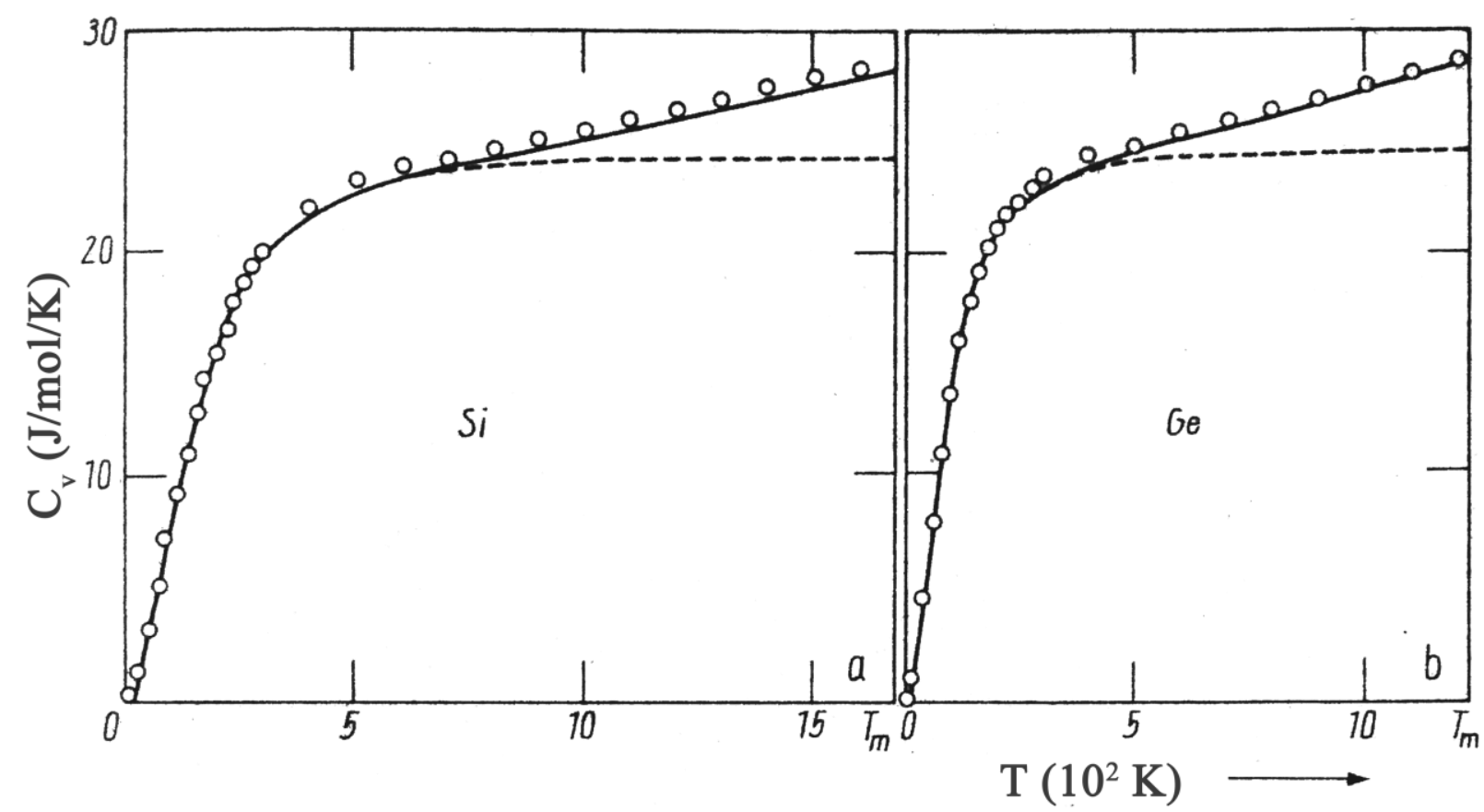

Figure 5.3. Specific heat of $\mathrm{Si}$ and Ge vs. temperature [135].

The specific heat was then converted to the heat capacity values in Table B by combining the lattice parameter data from section 5.1.4 with the specific heat data in Figure 5.3. Intermediate values were interpolated to generate the chart. 
Table B - Heat capacity table used with TDTR for $\mathrm{Si}_{1-\mathrm{x}} \mathrm{Ge}_{\mathrm{x}}$ vs. temperature (K).

\begin{tabular}{|c|c|c|c|c|c|c|c|c|c|c|}
\hline Composition & \multicolumn{8}{|c|}{$\mathbf{C}_{\mathbf{v}}\left(\mathbf{J} / \mathbf{c m}^{\mathbf{3}} / \mathbf{K}\right)$ at Temperature (K) } \\
\hline $\mathbf{S i}_{\mathbf{1 -}} \mathbf{G e}_{\mathbf{x}}$ & $\mathbf{3 0 0}$ & $\mathbf{4 0 0}$ & $\mathbf{5 0 0}$ & $\mathbf{6 0 0}$ & $\mathbf{7 0 0}$ & $\mathbf{8 0 0}$ & $\mathbf{9 0 0}$ & $\mathbf{1 0 0 0}$ & $\mathbf{1 1 0 0}$ & $\mathbf{1 2 0 0}$ \\
\hline $\mathbf{0 \%}$ & 1.661 & 1.874 & 1.980 & 2.033 & 2.066 & 2.096 & 2.131 & 2.169 & 2.208 & 2.247 \\
\hline $\mathbf{1 \%}$ & 1.661 & 1.874 & 1.980 & 2.033 & 2.066 & 2.096 & 2.130 & 2.169 & 2.208 & 2.247 \\
\hline $\mathbf{2 \%}$ & 1.662 & 1.874 & 1.980 & 2.033 & 2.066 & 2.096 & 2.130 & 2.168 & 2.208 & 2.247 \\
\hline $\mathbf{3 \%}$ & 1.663 & 1.875 & 1.980 & 2.033 & 2.065 & 2.096 & 2.130 & 2.168 & 2.208 & 2.247 \\
\hline $\mathbf{4 \%}$ & 1.663 & 1.875 & 1.980 & 2.032 & 2.065 & 2.095 & 2.129 & 2.168 & 2.208 & 2.247 \\
\hline $\mathbf{5 \%}$ & 1.664 & 1.875 & 1.980 & 2.032 & 2.065 & 2.095 & 2.129 & 2.167 & 2.207 & 2.247 \\
\hline $\mathbf{6 \%}$ & 1.665 & 1.875 & 1.979 & 2.032 & 2.065 & 2.095 & 2.129 & 2.167 & 2.207 & 2.247 \\
\hline $\mathbf{7 \%}$ & 1.665 & 1.875 & 1.979 & 2.032 & 2.065 & 2.095 & 2.128 & 2.167 & 2.207 & 2.247 \\
\hline $\mathbf{8 \%}$ & 1.666 & 1.875 & 1.979 & 2.032 & 2.065 & 2.094 & 2.128 & 2.166 & 2.207 & 2.247 \\
\hline $\mathbf{9 \%}$ & 1.667 & 1.875 & 1.979 & 2.032 & 2.065 & 2.094 & 2.128 & 2.166 & 2.207 & 2.247 \\
\hline $\mathbf{1 0 \%}$ & 1.668 & 1.875 & 1.979 & 2.032 & 2.064 & 2.094 & 2.128 & 2.166 & 2.206 & 2.247 \\
\hline $\mathbf{1 1 \%}$ & 1.668 & 1.875 & 1.979 & 2.032 & 2.064 & 2.094 & 2.127 & 2.165 & 2.206 & 2.247 \\
\hline $\mathbf{1 2 \%}$ & 1.669 & 1.875 & 1.979 & 2.032 & 2.064 & 2.094 & 2.127 & 2.165 & 2.206 & 2.247 \\
\hline $\mathbf{1 3 \%}$ & 1.670 & 1.875 & 1.979 & 2.031 & 2.064 & 2.093 & 2.127 & 2.165 & 2.206 & 2.247 \\
\hline $\mathbf{1 4 \%}$ & 1.670 & 1.875 & 1.979 & 2.031 & 2.064 & 2.093 & 2.126 & 2.164 & 2.206 & 2.247 \\
\hline $\mathbf{1 5 \%}$ & 1.671 & 1.875 & 1.979 & 2.031 & 2.064 & 2.093 & 2.126 & 2.164 & 2.205 & 2.247 \\
\hline $\mathbf{1 6 \%}$ & 1.672 & 1.875 & 1.979 & 2.031 & 2.064 & 2.093 & 2.126 & 2.164 & 2.205 & 2.247 \\
\hline $\mathbf{1 7 \%}$ & 1.672 & 1.876 & 1.979 & 2.031 & 2.063 & 2.092 & 2.125 & 2.164 & 2.205 & 2.247 \\
\hline $\mathbf{1 8 \%}$ & 1.673 & 1.876 & 1.978 & 2.031 & 2.063 & 2.092 & 2.125 & 2.163 & 2.205 & 2.247 \\
\hline $\mathbf{1 9 \%}$ & 1.674 & 1.876 & 1.978 & 2.031 & 2.063 & 2.092 & 2.125 & 2.163 & 2.204 & 2.247 \\
\hline $\mathbf{2 0 \%}$ & 1.674 & 1.876 & 1.978 & 2.031 & 2.063 & 2.092 & 2.125 & 2.163 & 2.204 & 2.247 \\
\hline $\mathbf{3 0 \%}$ & 1.681 & 1.876 & 1.977 & 2.030 & 2.061 & 2.090 & 2.122 & 2.160 & 2.202 & 2.247 \\
\hline $\mathbf{5 0 \%}$ & 1.695 & 1.878 & 1.976 & 2.027 & 2.058 & 2.085 & 2.116 & 2.153 & 2.198 & 2.247 \\
\hline $\mathbf{1 0 0 \%}$ & 1.729 & 1.882 & 1.972 & 2.022 & 2.051 & 2.074 & 2.101 & 2.138 & 2.187 & 2.247 \\
\hline & & & & & & & & & \\
\hline
\end{tabular}

\subsection{Electrical Properties}

\subsubsection{Mobility}

The mobility of samples can be measured via the Hall effect. In non-degenerate semiconductors the Hall mobility $\left(\mu_{\mathrm{H}}\right)$ may not equal the drift mobility $\left(\mu_{\mathrm{d}}\right)$. This ratio $\left(\mu_{\mathrm{H}} / \mu_{\mathrm{d}}\right)$ is called the Hall factor and is dependent on the magnetic field strength used during measurement. At high fields (by $\mathrm{B} \approx 10 \mathrm{~T}$ ) the Hall factor converges to 1 . For Si-Ge systems it can vary from $\mathrm{p}-$ $\mathrm{Si}=0.73$ to $\mathrm{p}-\mathrm{Ge}=1.7$ but for most Si-like compositions it is near 1 . In the degenerate case, it is also assumed to be 1 [119]. 
Hall mobility as a function of carrier concentration and temperature was predicted using Vining's model [42], graphed in Figure 5.4 for n-type Si-Ge.

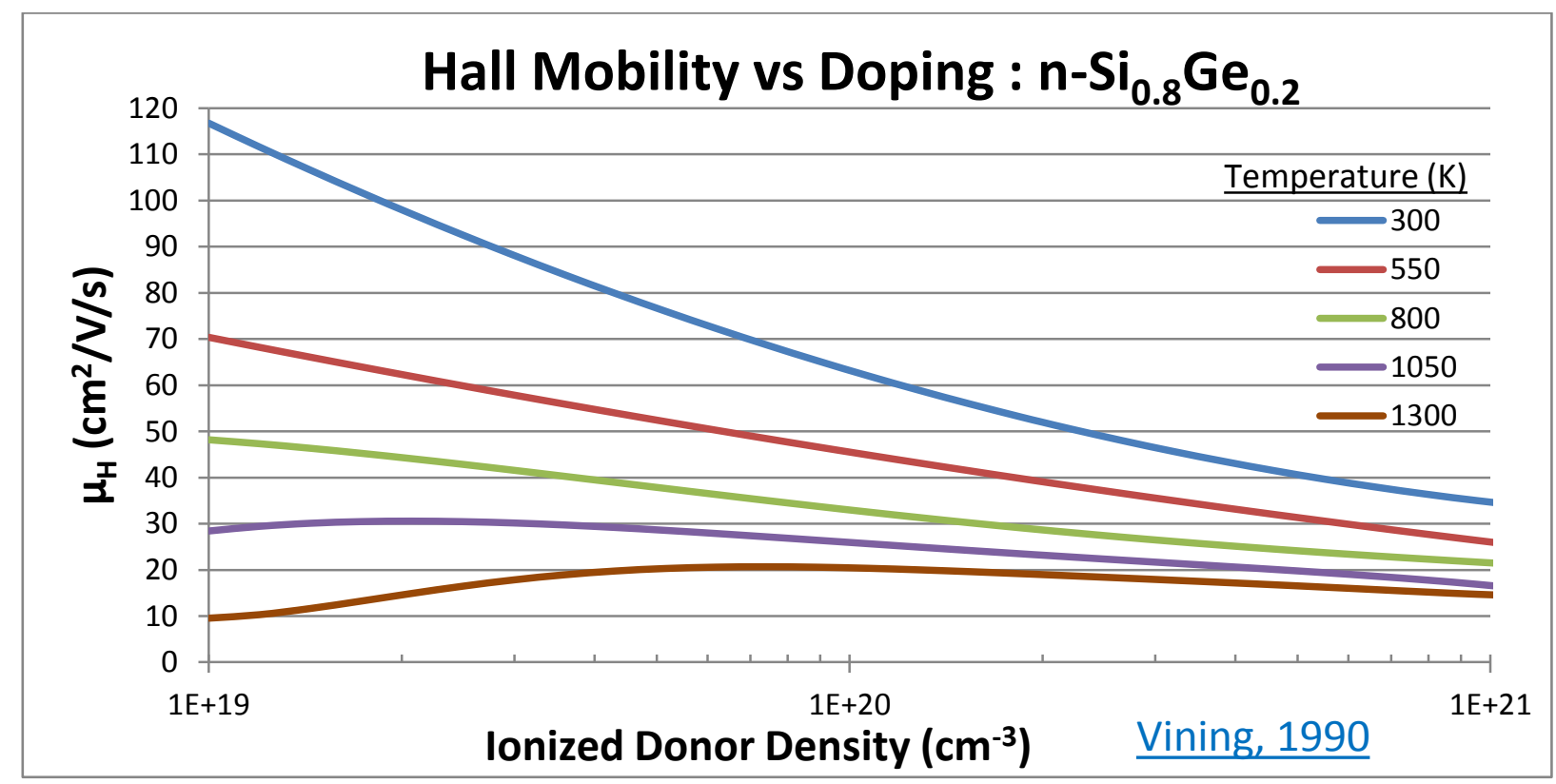

Figure 5.4. Si-Ge hall mobility dependence on carrier density at various temperatures [42].

Minnich et al. used XRD and TEM to determine that the grain boundary thickness for nano-Si-Ge is $\sim 1 \mathrm{~nm}$, relatively small compared to the typical electron wavelength of 6 to $11 \mathrm{~nm}$, thus grain boundary scattering tends to be coherent [17]. Figure $\mathbf{5 . 5}$ shows their modeling of mobility as a function of temperature. Using Matthiessen's rule [136], the individual scattering contributions are summed to fit the experimental data for nanograined $\mathrm{Si}_{0.8} \mathrm{Ge}_{0.2}$, with the $2.0 * 10^{20} / \mathrm{cm}^{3}$ phosphorus-doped material having a grain size of $\sim 12 \mathrm{~nm}$ and the $2.6^{*} 10^{20} / \mathrm{cm}^{3}$ boron-doped material having a grain size of $\sim 20 \mathrm{~nm}$. Due to the heavily degenerate doping, ionized impurity scattering (IIS) is the strongest scattering type at room temperature, but the nand p- types experience different contributions at higher temperatures. The n-type mobility continues to be primarily limited by IIS, whereas the p-type mobility initially has near equal contribution from grain boundary, phonon, and ionized impurity scattering but phonon scattering quickly dominates above room temperature. 

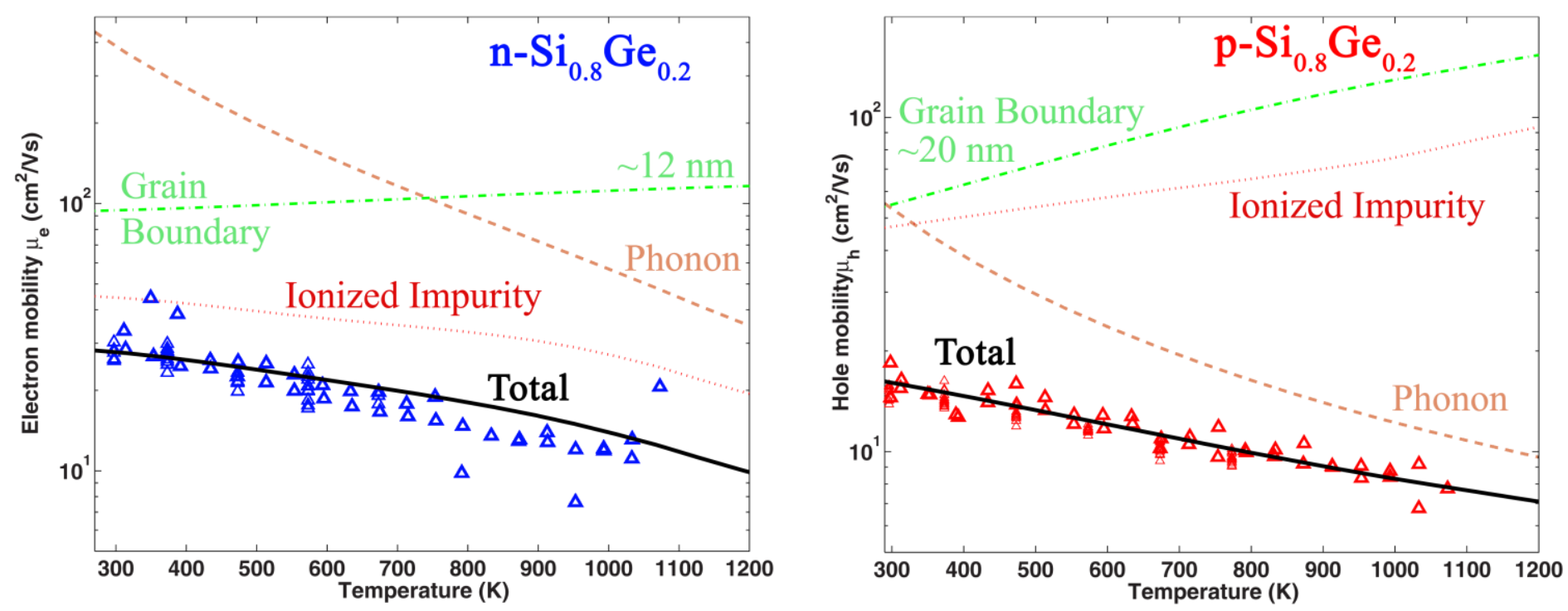

Figure 5.5. Scattering model of mobility vs. temperature for nano- $\mathrm{Si}_{0.8} \mathrm{Ge}_{0.2}$ [17].

\subsubsection{Resistivity}

The correlation between carrier concentration and resistivity has been established for both silicon and germanium for many years. The computation curves are viable for bulk materials, but may not offer high accuracy if the Si-Ge material is nano-grained or otherwise has oxide or porosity. Two Si-Ge curves are given in Figure 5.6 for computing the carrier concentration as a function of the measured resistivity for Si-Ge compositions of $\mathrm{Si}_{0.8} \mathrm{Ge}_{0.2}$ and $\mathrm{Si}_{0.98} \mathrm{Ge}_{0.02}$. The data are linear interpolations between the Thurber curves for Si [137] and those published for Ge by Cuttriss [138], but the composition doesn't make a large difference in the computation (e.g., the carrier concentration for $\mathrm{Si}_{0.5} \mathrm{Ge}_{0.5}$ is within a factor of 2 of pure $\mathrm{Si}$ ). 


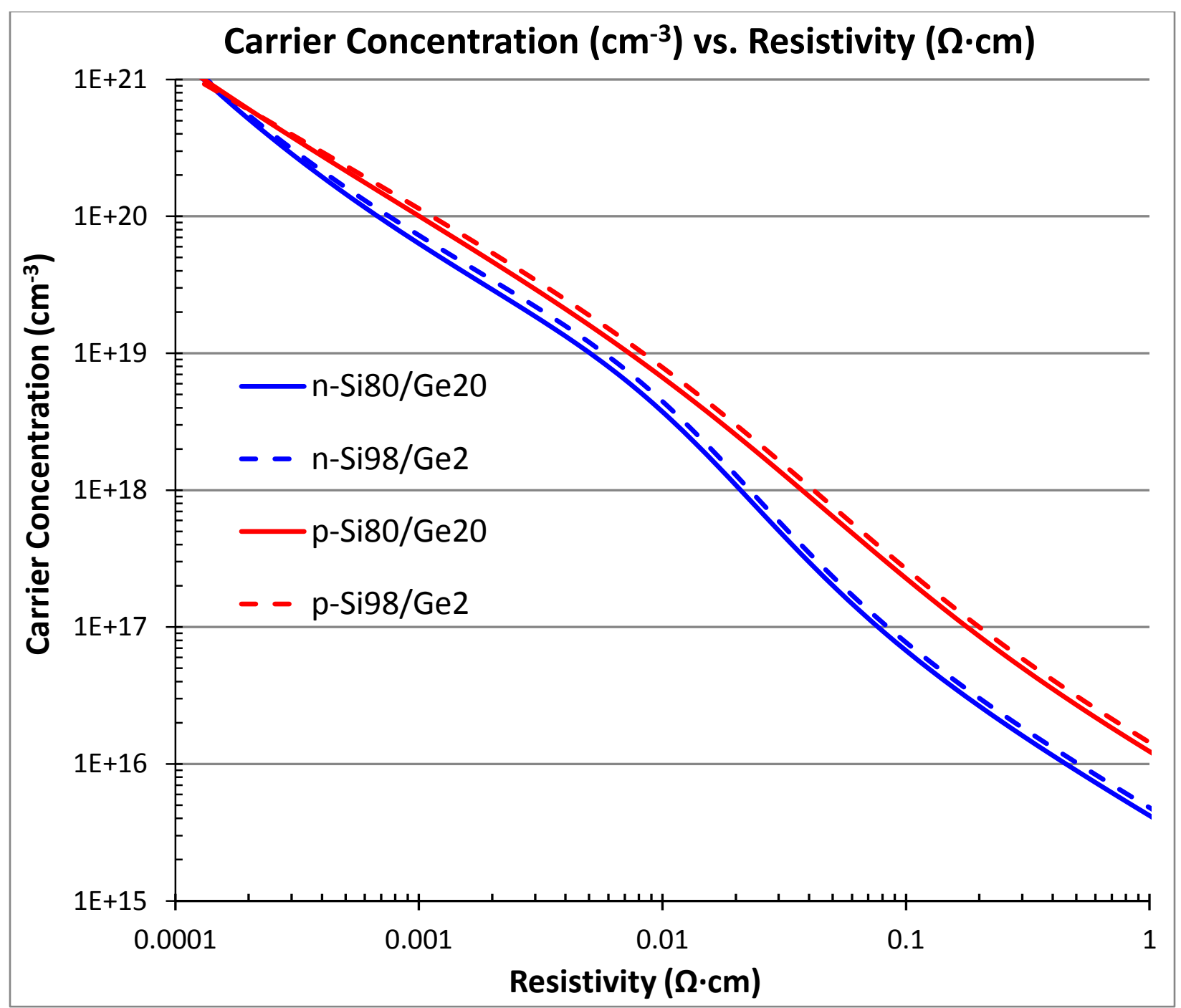

Figure 5.6. Carrier concentration vs. resistivity curve for Si-Ge $[137,138]$.

\subsubsection{Carrier Scattering Parameter}

The charge carrier scattering parameter is impacted by multiple variables, such as nanostructuring, temperature and doping level. Any change in how electrons migrate through a material may change the scattering parameter. $\mathbf{s}$ can be evaluated using Eq. 5-A once the relative strengths of different types of scattering methods are known for a given system. The three scattering methods accounted for here are Ionized Impurity (IIS), grain boundary (GB), and phonon. IIS is impacted by the level of doping, and tends to be the dominant method for super saturated n-Si-Ge. Grain boundary scattering is not as strong except for low temperature and low 
doping levels. Phonon scattering is strongest at high temperatures. By applying Matthiessen's rule for the individual scattering-type mobilities $\left(\mu_{\mathrm{IIS}}, \mu_{\mathrm{GB}}, \mu_{\mathrm{ph}}\right)$, a reasonable estimate can be made for the scattering parameter.

$$
s_{n, p}=\left(1.5 * \frac{\mu^{*}}{\mu_{\mathrm{IIS}}}\right)+\left(0.5 * \frac{\mu^{*}}{\mu_{\mathrm{GB}}}\right)+\left(-0.5 * \frac{\mu^{*}}{\mu_{p h}}\right)
$$

$s_{n, p}$ : scattering parameter for $n$ or $p$ type material, $\mu *$ : effective mobility;

$\mu_{\text {IIS: }}$ mobility from only ionized impurity scattering; $\mu_{\mathrm{GB}}$ : mobility from only grain boundary scattering;

$\mu_{\mathrm{ph}}$ : mobility from only phonon scattering.

Eq. 5-A. Scattering parameter model for n-type or p-type materials.

Based upon a carrier mobility study performed by Minnich et al. [17] where electron and hole scattering mechanisms were evaluated vs. temperature, and in combination with Vining's mobility models [42], the s parameter was evaluated for both n- and p-type Si-Ge materials for numerous conditions using Eq. 5-A. Only material composition was not varied, instead assuming $\mathrm{Si}_{0.8} \mathrm{Ge}_{0.2}$; this assumption causes error only at non-degenerate doping levels, and even then s only changes by a few hundredths if the material remains Si-like (i.e., $<85$ at\% Ge). The values create a matrix from which interpolation can be used for doping between $10^{18}$ and $10^{21}$ $/ \mathrm{cm}^{3}$, temperature from 300 to $1200 \mathrm{~K}$, and grain size from $10 \mathrm{~nm}$ to $1 \mu \mathrm{m}$. This window covers nearly all applicable conditions for Si-Ge thermoelectrics. As this chart bridges theory with practice, it should be used only as a first order estimate, though it's certainly better than the alternative of just assuming $\mathbf{s}=1 / 2$. 


\begin{tabular}{|c|r|r|r|r|}
\hline s_n & & \multicolumn{2}{|c|}{ Grain size $(\mathrm{m}):$} & $1 \mathrm{E}-06$ \\
\hline $\mathrm{n} / \mathrm{cm}^{3}$ & $1 \mathrm{E}+18$ & $1 \mathrm{E}+19$ & $1 \mathrm{E}+20$ & $1 \mathrm{E}+21$ \\
\hline $\mathrm{T}(\mathrm{K})$ & & & & \\
\hline 300 & 0.34 & 0.71 & 1.09 & 1.49 \\
\hline 400 & 0.26 & 0.64 & 1.03 & 1.44 \\
\hline 500 & 0.17 & 0.56 & 0.97 & 1.38 \\
\hline 600 & 0.09 & 0.48 & 0.91 & 1.33 \\
\hline 700 & 0.00 & 0.41 & 0.85 & 1.27 \\
\hline 800 & -0.07 & 0.34 & 0.79 & 1.22 \\
\hline 900 & -0.13 & 0.28 & 0.74 & 1.17 \\
\hline 1000 & -0.18 & 0.23 & 0.68 & 1.11 \\
\hline 1100 & -0.23 & 0.18 & 0.63 & 1.06 \\
\hline 1200 & -0.28 & 0.13 & 0.57 & 1.00 \\
\hline
\end{tabular}

\begin{tabular}{|c|r|r|r|r|}
\hline s_p & & \multicolumn{2}{|c|}{ Grain size $(\mathrm{m}):$} & $1 \mathrm{E}-06$ \\
\hline $\mathbf{p ~ / \mathbf { c m } ^ { 3 }}$ & $1 \mathrm{E}+18$ & $1 \mathrm{E}+19$ & $1 \mathrm{E}+20$ & $1 \mathrm{E}+21$ \\
\hline $\mathbf{T}(\mathrm{K})$ & & & & \\
\hline 300 & -0.10 & 0.10 & 0.38 & 0.64 \\
\hline 400 & -0.20 & -0.01 & 0.25 & 0.50 \\
\hline 500 & -0.27 & -0.11 & 0.12 & 0.36 \\
\hline 600 & -0.34 & -0.20 & -0.01 & 0.22 \\
\hline 700 & -0.39 & -0.27 & -0.12 & 0.10 \\
\hline 800 & -0.43 & -0.33 & -0.20 & 0.00 \\
\hline 900 & -0.46 & -0.37 & -0.26 & -0.08 \\
\hline 1000 & -0.48 & -0.40 & -0.31 & -0.14 \\
\hline 1100 & -0.50 & -0.42 & -0.34 & -0.19 \\
\hline 1200 & -0.50 & -0.44 & -0.37 & -0.23 \\
\hline
\end{tabular}

\begin{tabular}{|c|r|r|r|r|}
\hline s_n & & \multicolumn{2}{|c|}{ Grain size $(\mathrm{m}):$} & $1 \mathrm{E}-07$ \\
\hline $\mathrm{n} / \mathrm{cm}^{3}$ & $1 \mathrm{E}+18$ & $1 \mathrm{E}+19$ & $1 \mathrm{E}+20$ & $1 \mathrm{E}+21$ \\
\hline $\mathrm{T}(\mathrm{K})$ & & & & \\
\hline 300 & 0.37 & 0.70 & 1.03 & 1.39 \\
\hline 400 & 0.30 & 0.64 & 0.98 & 1.34 \\
\hline 500 & 0.23 & 0.58 & 0.93 & 1.30 \\
\hline 600 & 0.16 & 0.52 & 0.88 & 1.25 \\
\hline 700 & 0.09 & 0.46 & 0.84 & 1.21 \\
\hline 800 & 0.03 & 0.40 & 0.79 & 1.17 \\
\hline 900 & -0.03 & 0.34 & 0.74 & 1.13 \\
\hline 1000 & -0.09 & 0.29 & 0.69 & 1.08 \\
\hline 1100 & -0.15 & 0.23 & 0.64 & 1.03 \\
\hline 1200 & -0.20 & 0.18 & 0.59 & 0.99 \\
\hline
\end{tabular}

\begin{tabular}{|c|r|r|r|r|}
\hline s_p & & \multicolumn{2}{|c|}{ Grain size $(\mathrm{m}):$} & $1 \mathrm{E}-07$ \\
\hline $\mathbf{p ~ / \mathbf { c m } ^ { 3 }}$ & $1 \mathrm{E}+18$ & $1 \mathrm{E}+19$ & $1 \mathrm{E}+20$ & $1 \mathrm{E}+21$ \\
\hline $\mathbf{T}(\mathbf{K})$ & & & & \\
\hline 300 & 0.03 & 0.19 & 0.40 & 0.61 \\
\hline 400 & -0.07 & 0.07 & 0.27 & 0.48 \\
\hline 500 & -0.16 & -0.04 & 0.14 & 0.35 \\
\hline 600 & -0.25 & -0.14 & 0.03 & 0.23 \\
\hline 700 & -0.32 & -0.22 & -0.08 & 0.12 \\
\hline 800 & -0.37 & -0.29 & -0.16 & 0.02 \\
\hline 900 & -0.41 & -0.34 & -0.23 & -0.06 \\
\hline 1000 & -0.45 & -0.37 & -0.28 & -0.12 \\
\hline 1100 & -0.47 & -0.40 & -0.32 & -0.18 \\
\hline 1200 & -0.50 & -0.43 & -0.35 & -0.22 \\
\hline \multicolumn{7}{|r}{} \\
\hline
\end{tabular}

\begin{tabular}{|c|c|c|c|c|}
\hline s_n & & \multicolumn{2}{|c|}{ Grain size $(\mathrm{m}):$} & $1 \mathrm{E}-08$ \\
\hline $\mathrm{n} / \mathrm{cm}^{3}$ & $1 \mathrm{E}+18$ & $1 \mathrm{E}+19$ & $1 \mathrm{E}+20$ & $1 \mathrm{E}+21$ \\
\hline $\mathrm{T}(\mathrm{K})$ & & & & \\
\hline 300 & 0.41 & 0.70 & 0.98 & 1.30 \\
\hline 400 & 0.35 & 0.65 & 0.94 & 1.26 \\
\hline 500 & 0.28 & 0.59 & 0.90 & 1.23 \\
\hline 600 & 0.22 & 0.54 & 0.87 & 1.20 \\
\hline 700 & 0.15 & 0.49 & 0.84 & 1.17 \\
\hline 800 & 0.09 & 0.44 & 0.80 & 1.14 \\
\hline 900 & 0.04 & 0.38 & 0.75 & 1.10 \\
\hline 1000 & -0.02 & 0.33 & 0.71 & 1.06 \\
\hline 1100 & -0.08 & 0.28 & 0.66 & 1.02 \\
\hline 1200 & -0.14 & 0.22 & 0.62 & 0.97 \\
\hline
\end{tabular}

\begin{tabular}{|c|r|r|r|r|}
\hline s_p & & \multicolumn{2}{|c|}{ Grain size $(\mathrm{m}):$} & $1 \mathrm{E}-08$ \\
\hline $\mathbf{p} / \mathrm{cm}^{3}$ & $1 \mathrm{E}+18$ & $1 \mathrm{E}+19$ & $1 \mathrm{E}+20$ & $1 \mathrm{E}+21$ \\
\hline $\mathbf{T}(\mathrm{K})$ & & & & \\
\hline 300 & 0.16 & 0.29 & 0.48 & 0.66 \\
\hline 400 & 0.06 & 0.18 & 0.35 & 0.53 \\
\hline 500 & -0.04 & 0.06 & 0.22 & 0.41 \\
\hline 600 & -0.13 & -0.04 & 0.10 & 0.28 \\
\hline 700 & -0.21 & -0.13 & -0.01 & 0.17 \\
\hline 800 & -0.28 & -0.20 & -0.09 & 0.08 \\
\hline 900 & -0.33 & -0.26 & -0.16 & 0.00 \\
\hline 1000 & -0.36 & -0.30 & -0.21 & -0.06 \\
\hline 1100 & -0.39 & -0.33 & -0.25 & -0.12 \\
\hline 1200 & -0.43 & -0.36 & -0.29 & -0.17 \\
\hline
\end{tabular}

Table C - Scattering parameter estimation for Si-Ge using temperature, doping, and grain size. 


\subsubsection{Lorenz Number}

Flage-Larsena and Prytz have modeled the Lorenz number for heavily doped semiconductors assuming parabolic band behavior as a function of temperature and doping level, as shown in Figure 5.7 [139]. The modeling shows the Lorenz number goes to the classical value for metals at the heaviest doping levels, but that at relatively light doping (for thermoelectrics) the value can deviate up to $20 \%$ higher or $40 \%$ lower if the carrier scattering type is ionized impurity or acoustic, respectively.

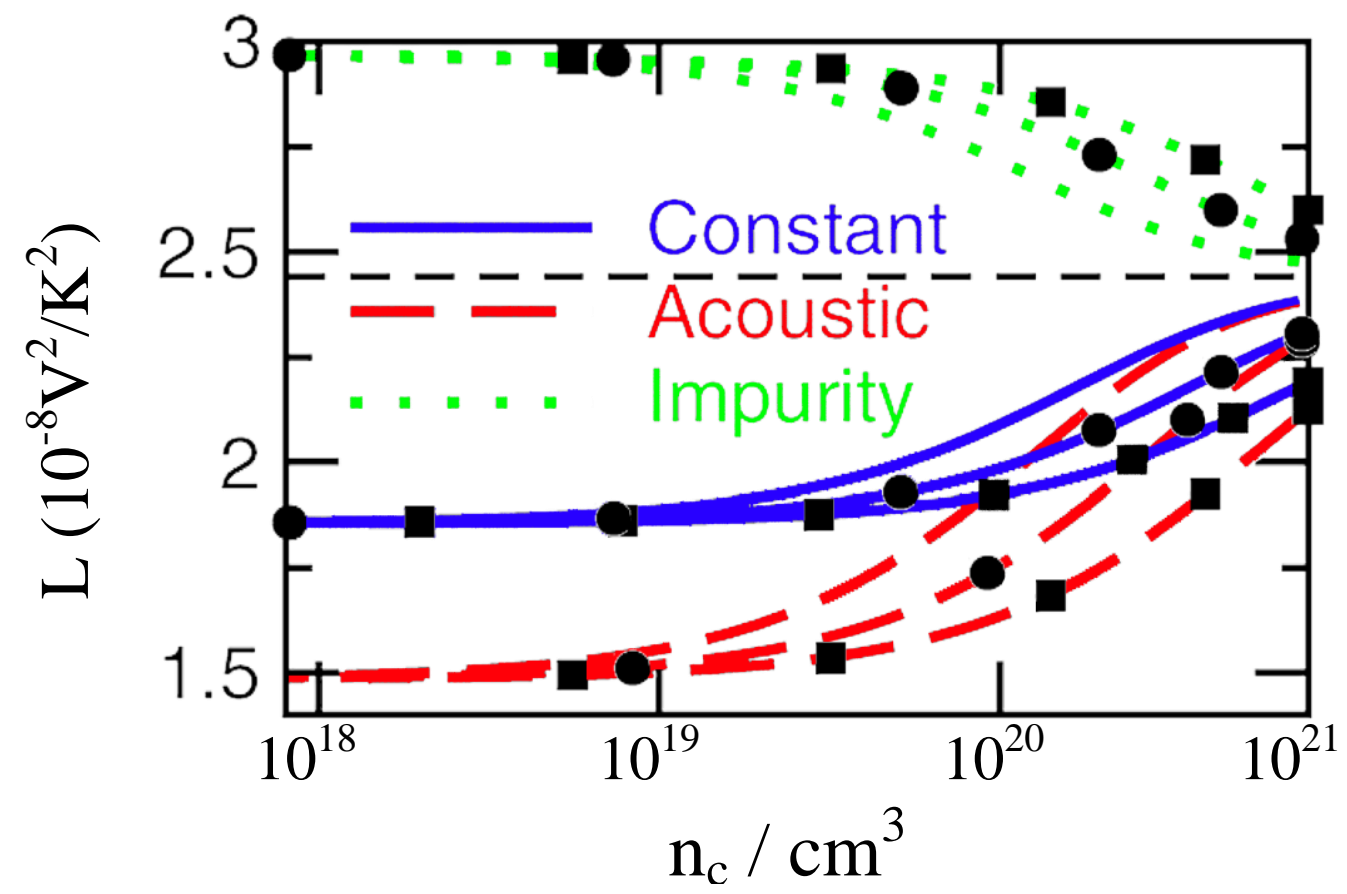

Figure 5.7. Lorenz value vs. doping at 300, 500 (•), and 800 (匹) K [139].

\subsubsection{Energy Bandgap}

In thermoelectric applications, the bandgap is an important parameter. Without a bandgap, the material is metallic and unable to support a significant Seebeck coefficient. Too small of a bandgap results in poor high temperature performance as intrinsic thermal excitation 
of minority carriers decreases the Seebeck and increases the thermal conductivity [42,59]. Alternatively, too large of a bandgap would impede good electrical conductivity, with the limit being an insulator.

Silicon has a relatively large energy bandgap for thermoelectric usage, $E_{G}=E_{c}-E_{v}=$ $1.12 \mathrm{eV}$ as in Figure 5.8, where $E_{c}$ is the band edge for electrons and $E_{v}$ is the band edge for holes. Alloying with germanium not only greatly reduces thermal conductivity, but also reduces the bandgap by $0.12 \mathrm{eV}$ for 20 at\% Ge; moreover, for the p-type material Ge also reduces resistivity by improving B activation and hole mobility [140]. The bandgap remains relatively large even after doping, which allows Si-Ge to function best at high temperatures by constraining the generation of minority carriers.

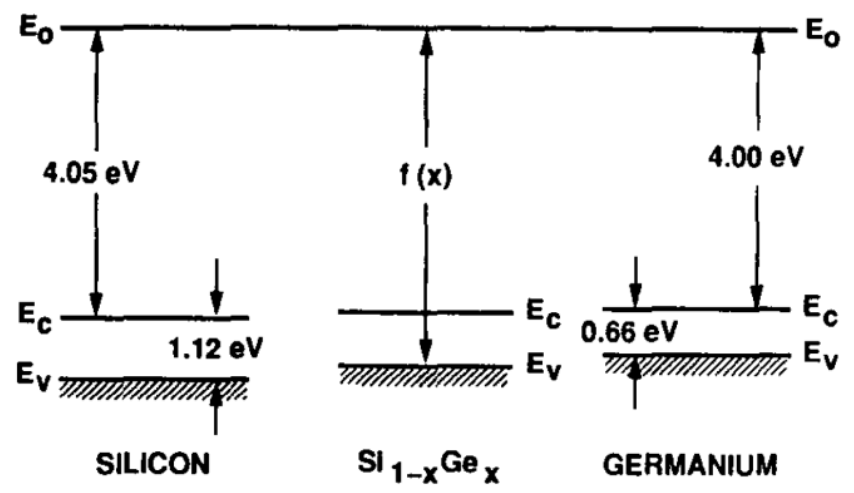

Figure 5.8. Electron work functions and bandgaps for Si-Ge [140].

Krishnamurthy et al. published the Si-Ge alloy bandgap in 1985 at room temperature [141]. Vining refined this curve in 1990 [42], and his curve is chosen here as the basis for calculating $\mathrm{E}_{\mathrm{g}}$ as a function of temperature and doping level, graphed in Figure 5.9. 


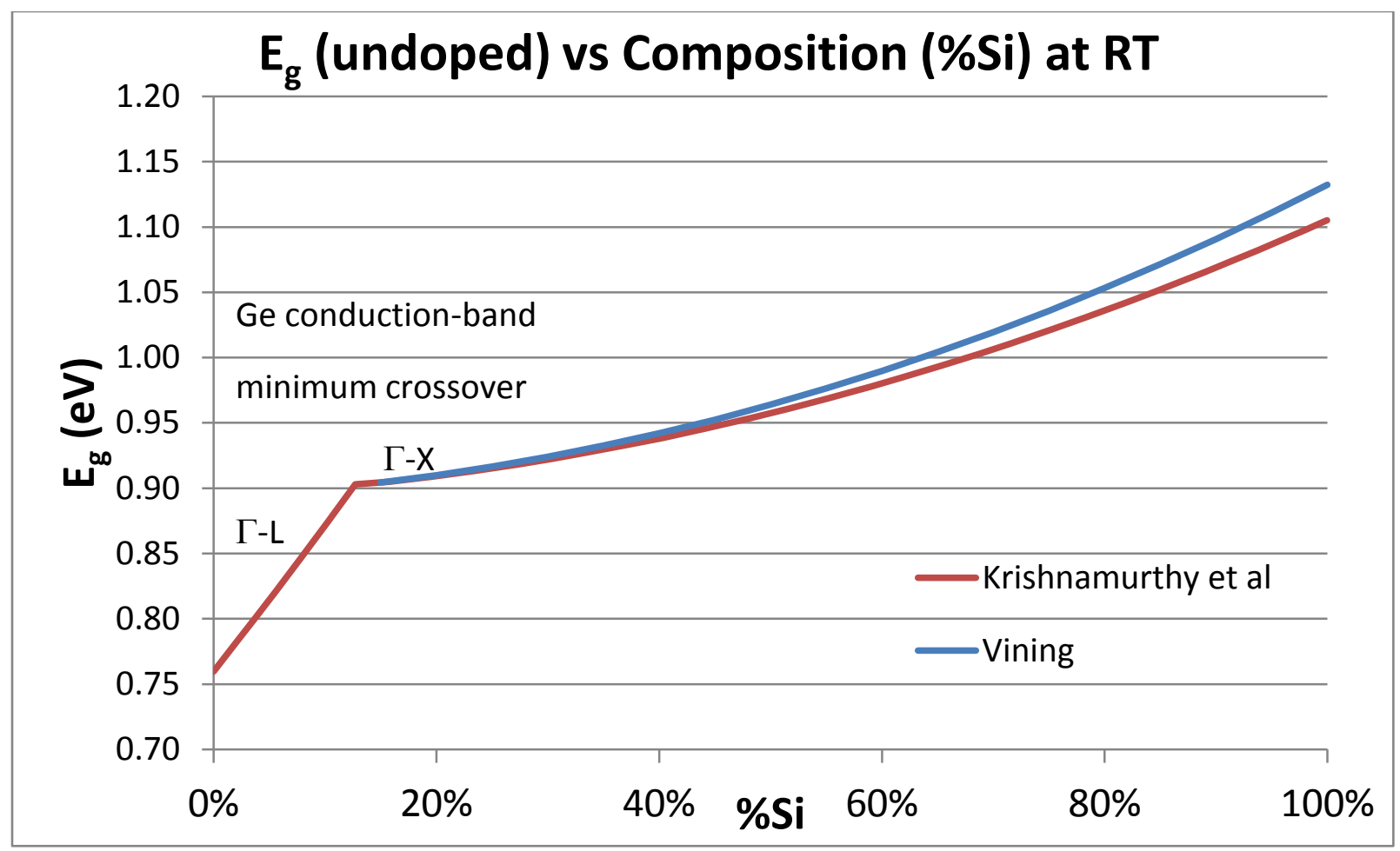

Figure 5.9. Energy band gap of Si-Ge alloys.

\subsubsection{Dopant Supersaturation}

Silicon germanium has a limited solubility for boron and phosphorus which impacts the maximum activated dopant. Minnich et al. have determined the carrier concentration for both nand p- type $\mathrm{Si}_{0.8} \mathrm{Ge}_{0.2}$ as a function of temperature, given the dopant is supersaturated at room temperature [17], as shown in Figure 5.10. The precipitation of phosphorus at intermediate temperatures has long been established [42] and does not appear to be correlated to the grain size, but solubility is improved with the addition of Ge [61]. The boron does not precipitate until $\sim 1000 \mathrm{~K}$, and the solubility limit does not tend to increase at higher temperatures like P. 


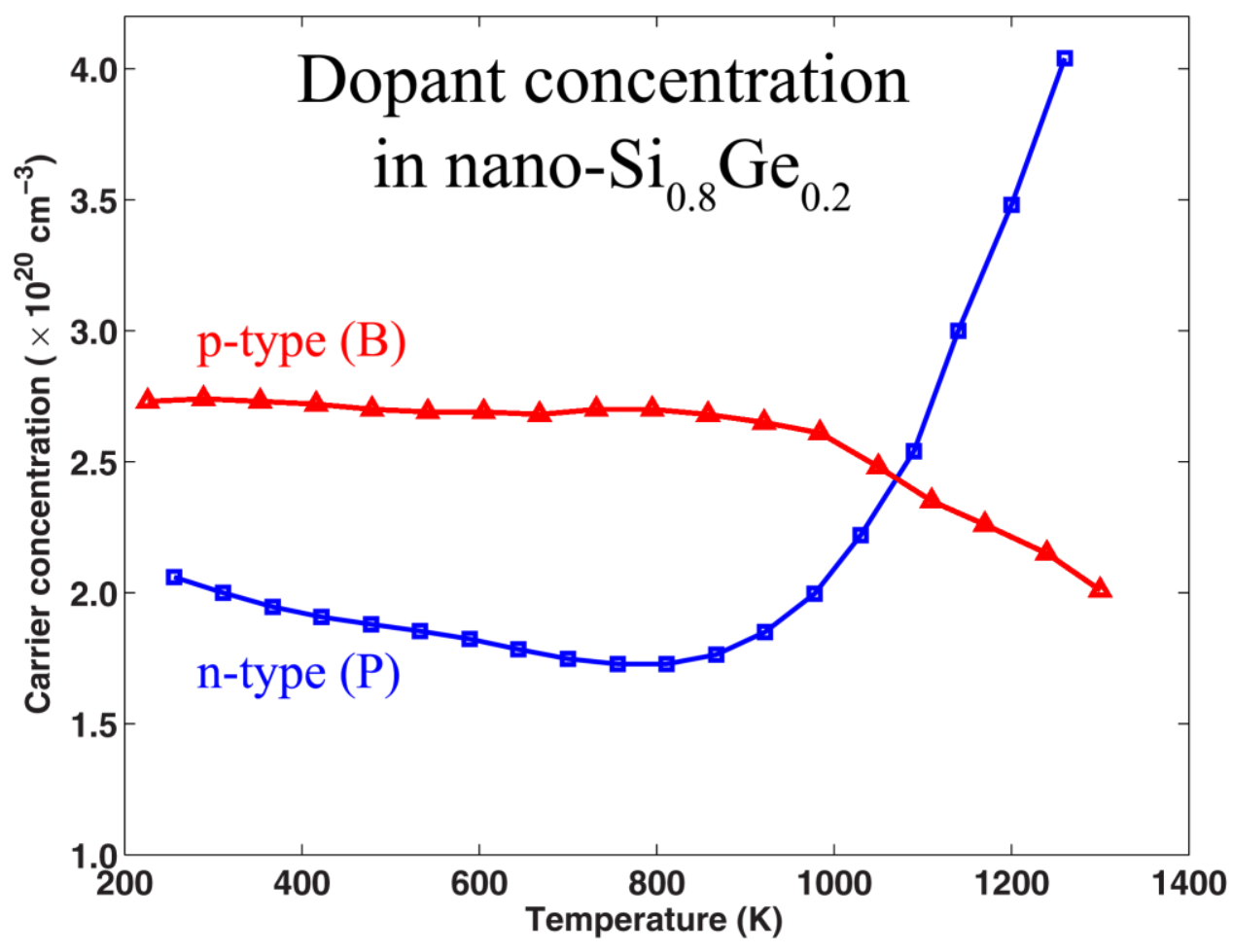

Figure 5.10. Carrier concentration vs. temperature for nano-Si-Ge [17]. 


\section{Chapter 6 : Results and Discussions}

Section 6.1.1 characterizes the purchased powders of silicon, germanium, and boron, with the laser sintering results presented in section 7.1. The doped $\mathrm{Si}-\mathrm{Ge}$ nanoclusters provided by the University of Minnesota are characterized in section 6.1.2 with the laser sintering results detailed in the rest of this chapter and RTA results provided in section 7.2.

\subsection{Raw Particle and Film Characterization}

\subsubsection{Raw Powders}

The silicon nanopowders purchased from NanoAmor were specified to have an average particle size of $50 \mathrm{~nm}$. The raw density was very low until compaction during mixing with the germanium powder. The SEM image in Figure 6.1 shows some agglomeration and a wide range of sizes, but the size appears to match the $50 \mathrm{~nm}$ specification. The starting powder was analyzed in both SEM and TEM via EDS, as shown in Table D. SEM/EDS indicated a large oxygen concentration even before heating. The high oxygen concentration was also observed in the TEM/EDS, although after running a standard on $\mathrm{SiO}_{2}$ for calibration purposes, the reported oxygen contamination was much lower at 19 at\%, albeit still very significant. Note also that some carbon was seen in the SEM/EDS, which was likely from the sample holder, but contamination during production of the silicon nano-powder cannot be ruled out.

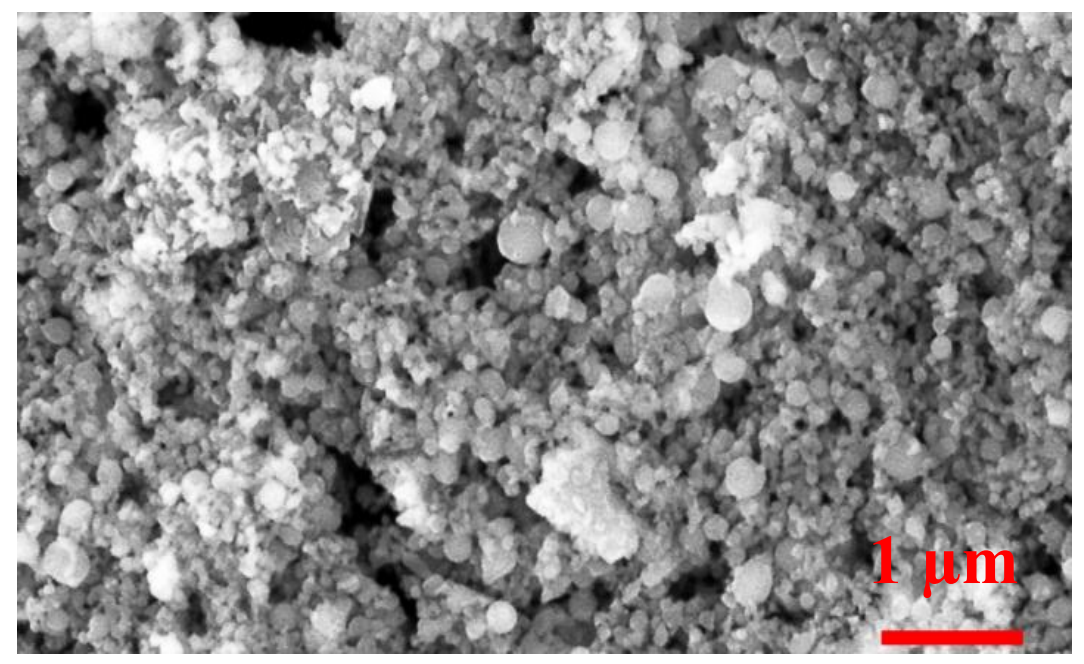

Figure 6.1. Silicon nanopowder image from SEM. 
Table D. Silicon nanoparticle composition from EDS in SEM and TEM.

\begin{tabular}{|c|c|c|c|c|c|c|c|}
\hline SEM & Nano-Si & TEM & $\alpha-\mathrm{SiO}_{2}$ & Nano-Si & TEM & $\alpha-\mathrm{SiO}_{2}$ & Nano-Si \\
\hline & $\begin{array}{c}\text { Uncalibrated } \\
\text { at } \%\end{array}$ & & $\begin{array}{c}\text { Uncalibrated } \\
\text { at } \%\end{array}$ & $\begin{array}{c}\text { Uncalibrated } \\
\text { at } \%\end{array}$ & & $\begin{array}{c}\text { Calibrated } \\
\text { at } \%\end{array}$ & $\begin{array}{c}\text { Calibrated } \\
\text { at } \%\end{array}$ \\
\hline Si K & 69.4 & Si K & 16.8 & 42.4 & Si K & 33.3 & 81.1 \\
\hline O K & 26.5 & O K & 83.2 & 57.6 & O K & 66.7 & 18.9 \\
\hline C K & 4 & C K & . & - & C K & - & - \\
\hline
\end{tabular}

Throughout the study of silicon nanoparticles, some level of oxidation is nearly always present. The high surface area to volume ratio and 1-2 nm native oxide shell allow significant oxygen adsorption in terms of atomic percent. The silica decreases electrical conductivity; although the viscous flow of silica during sintering has improved densification using certain sintering methods [142], it tends to inhibit particle necking and substrate wetting during laser sintering. Analysis of the powder using SEM/EDS [Table E] and XRD [Figure 6.2] was performed after mixing with germanium and boron but before laser sintering. XRD of the sample after induction heating at 200 amps but before laser sintering is shown in Figure 6.3. In this case, note that both the Ge (111) and (220) peaks have disappeared, indicating that the Ge has begun alloying with the $\mathrm{Si}$ due solely to preheating. This provides some indication of the temperature experienced when using the induction heating and the ability to stimulate densification. For reference, with the same molybdenum substrate configuration, pure $150 \mathrm{~nm}$ Ge melts in the induction heater when set to 300 amps within a few minutes. Otherwise, the XRD peaks are as expected for $\mathrm{Si}$ and $\mathrm{Ge}$, ignoring the sharp peaks from the Mo substrate and the $\mathrm{SiO}_{2}$ impurity.

Table E. Silicon germanium nanopowder composition from EDS in SEM.

\begin{tabular}{|c|c|}
\hline EDS/SEM & Si-Ge powder \\
\hline & Uncalibrated at\% \\
\hline Si K & 59 \\
Ge L & 8 \\
O K & 34 \\
\hline
\end{tabular}




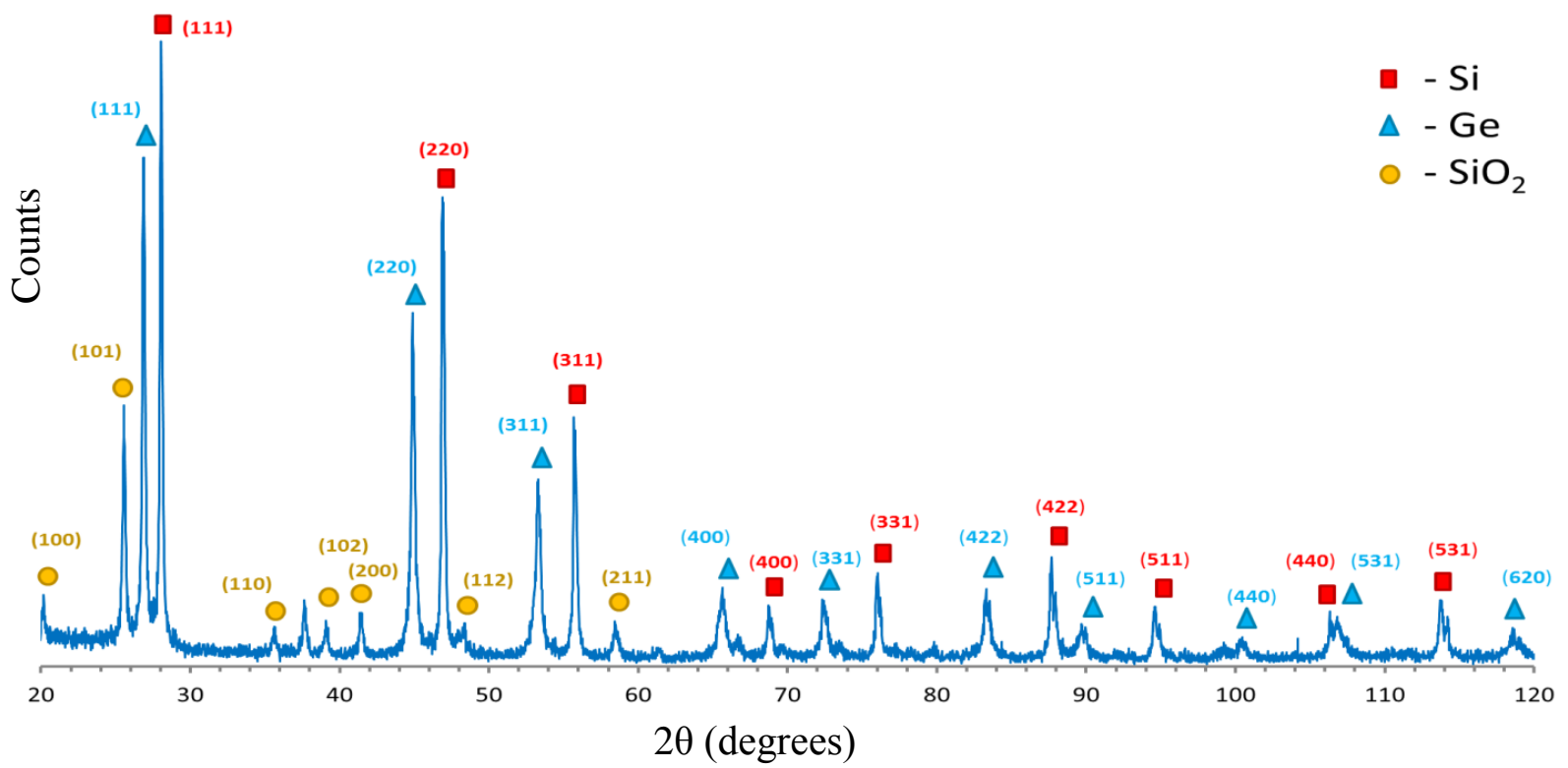

Figure 6.2. XRD phase analysis of Si-Ge nanopowder before sintering.

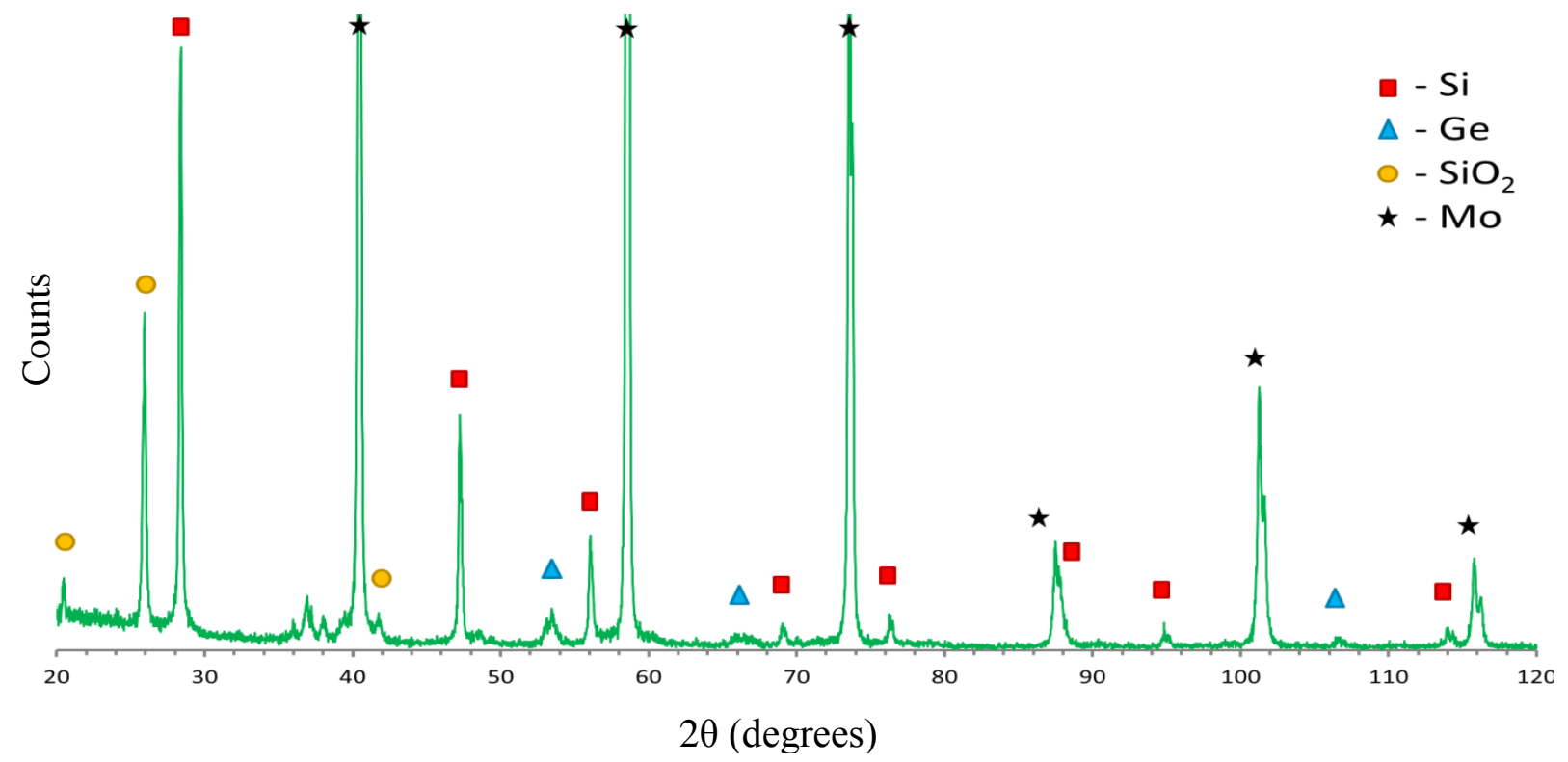

Figure 6.3. XRD phase analysis of Si-Ge nanopowder after only induction heating. 


\subsubsection{Plasma-Deposited Nanocluster Thin Films}

The non-thermal plasma films were supplied by Prof. Uwe Kortshagen's group at the University of Minnesota [48]. Any references in this work to thin films or nanoclusters indicate materials supplied by UMN. PhD candidate David Rowe fabricated the films using a custom plasma deposition chamber, which provided substantial control over the final particle and film morphology and composition. Figure 6.4 shows an example of unprocessed Si-Ge nanoclusters on a silicon substrate provided by Prof. Kortshagen's group. The film has reasonable starting density of $\sim 30 \%$ of the bulk value, good adhesion to the substrate, and low thickness variation.

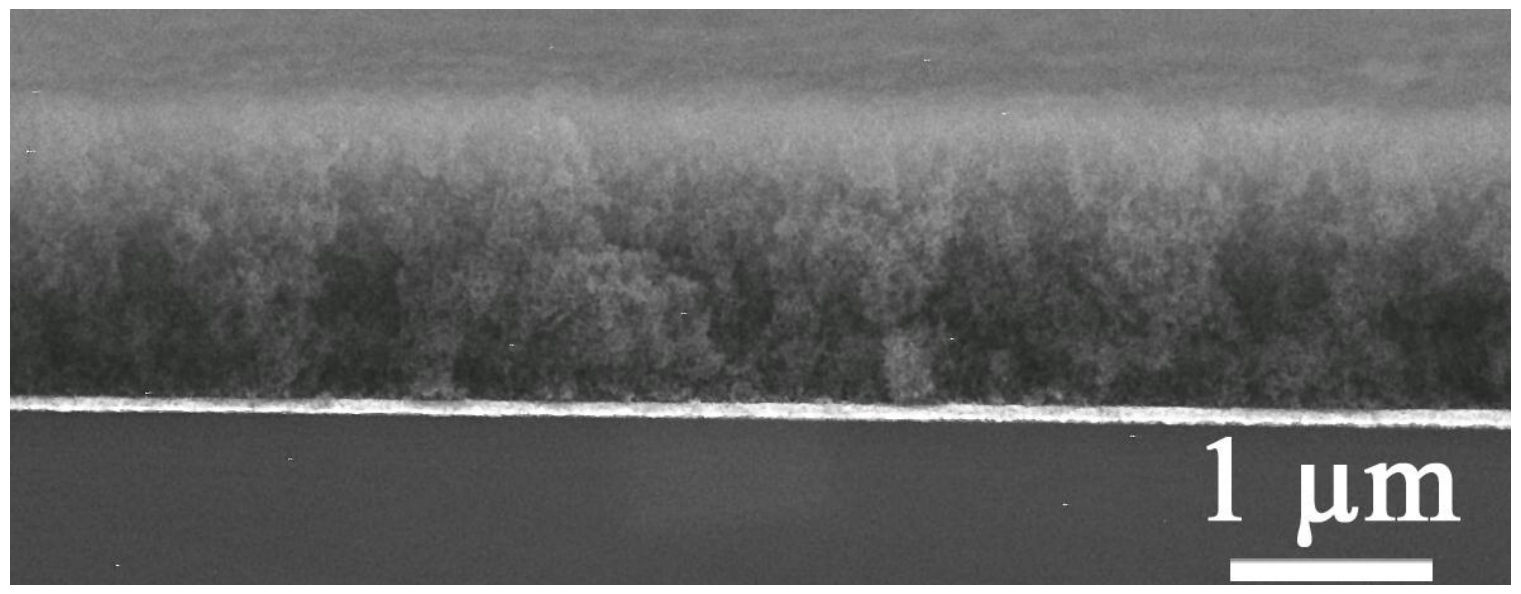

Figure 6.4. SEM of raw Si-Ge nanocluster film showing a cross-section [48].

Note that as the rate of film deposition increases, clumping of particles caused roughening of the surface, as shown in Figure 6.5. This morphology creates problems during laser sintering, as the laser isn't able to be dynamically adjusted to account for the sudden thickness change, which can prevent sintering of the region underneath the agglomerates and generally increases the surface roughness of the sintered film. UMN modified the deposition process to improve the surface smoothness. Figure 6.6 shows the EDS spectra of the as-deposited film using in the SEM. The atomic composition measured $67 \% \mathrm{Si}, 6 \% \mathrm{Ge}$, and $27 \% \mathrm{O}$, however since the EDS is not calibrated for this material the accuracy appears to be poor. Standardless EDS consistently under-reported $\mathrm{Ge}$ and over-reported $\mathrm{O}$, as compared with multiple characterization techniques in the UMN labs, including EDS and XRD. Regardless, oxidation of the nanoclusters is definitely a concern during handling. After opening the vacuum sealed bags, the samples were immediately placed into the laser chamber for processing, in an effort to minimize oxidation. 
The samples are somewhat protected by hydrogen surface passivation, but based on the EDS peak it does not appear to be sufficient for complete oxidation prevention.

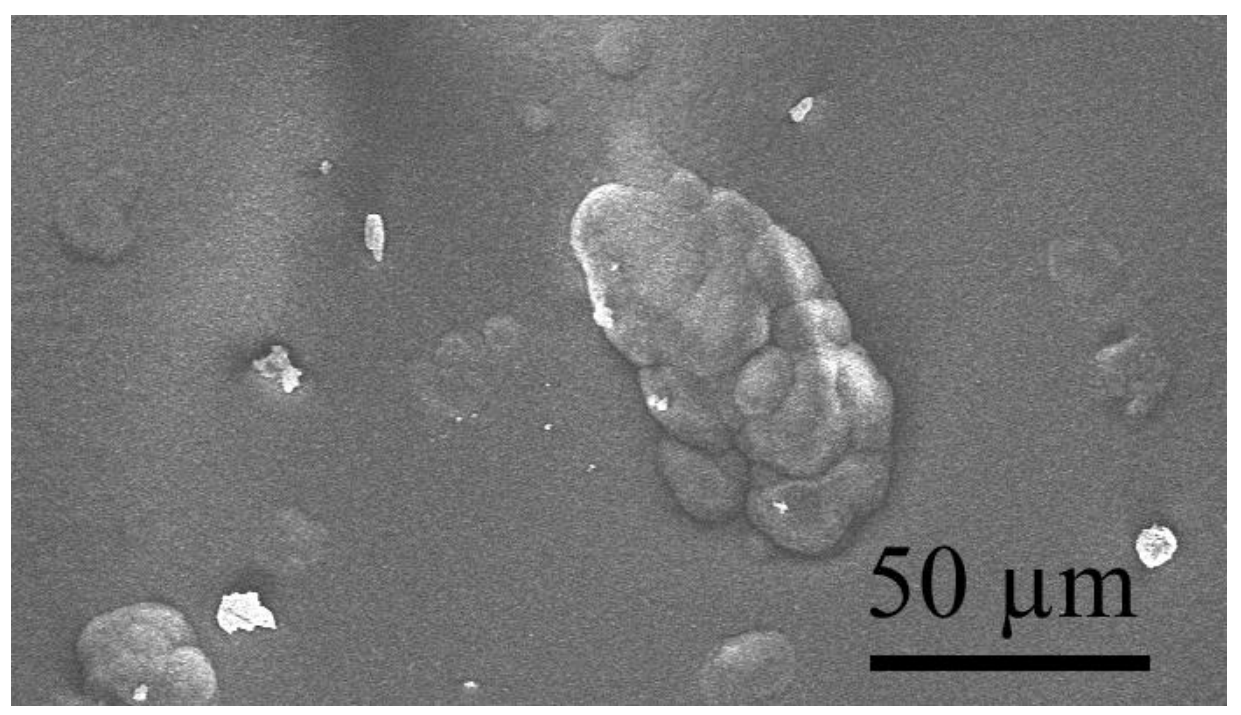

Figure 6.5. SEM of Si-Ge nanocluster surface with agglomeration before process modification.

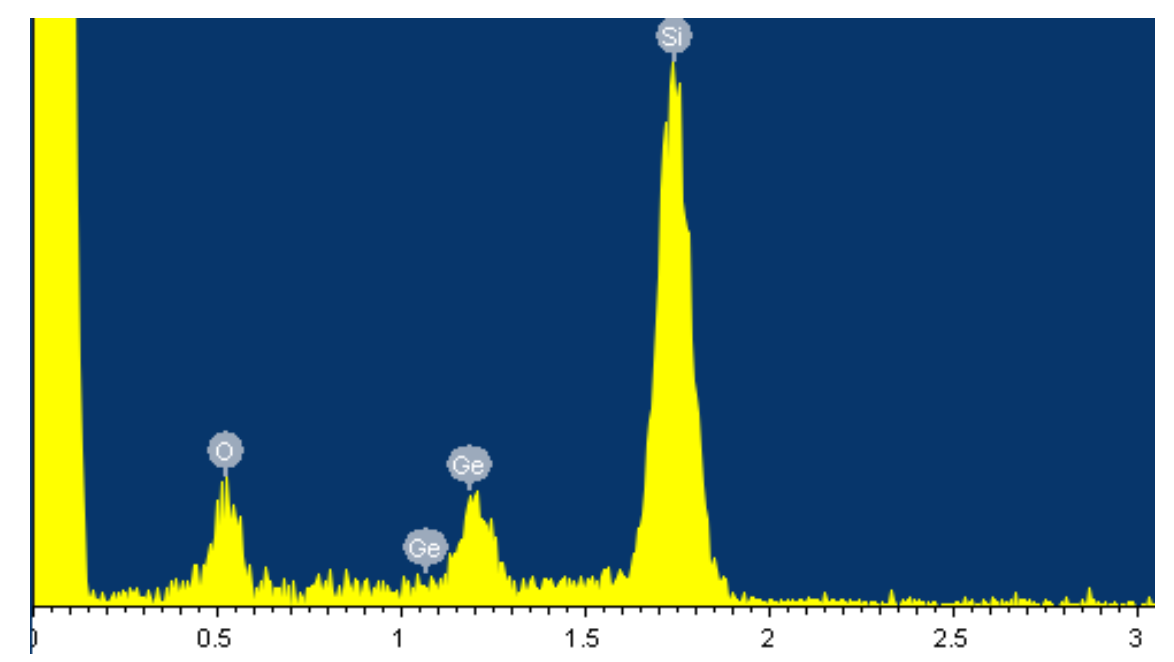

Figure 6.6. EDS of the raw Si-Ge nanocluster film, showing some oxygen impurity.

After improving the deposition process, the films appear denser and more solid-like. Scratching the surface actually causes flaking of the film rather than just deformation, as shown in Figure 6.7 [48]. Furthermore, by taking XRD of the raw films, UMN was able to confirm that the deposition process alloys the nanoclusters at the particle level. Figure 6.8 [unpublished data] [112] shows the (111) reflection of the four conditions tested: 1) pure $\mathrm{Si}, 2$ ) pure $\mathrm{Ge}, 3$ ) individual layers of $\mathrm{Si}$ and $\mathrm{Ge}$, and 4) co-deposited $\mathrm{Si}$ and $\mathrm{Ge}$. By simultaneously flowing silane $\left(\mathrm{SiH}_{4}\right)$ and germane $\left(\mathrm{GeH}_{4}\right)$ in the plasma chamber, the atoms naturally alloy as they begin 
clustering in flight and before impacting the substrate. The combined XRD peak for Si-Ge alloy was shifted from the pure Si peak, and by using Vegard's law UMN confirmed the composition was $\mathrm{Si}_{0.8} \mathrm{Ge}_{0.2}$. Likewise, the boron or phosphorus was added in the plasma reaction chamber for doping of the Si-Ge nanoclusters; the boron tends to be located nearer the interior of the nanoclusters, while the phosphorus tends to be in the outer shell [110]. UMN produced the composition of nanoclusters for laser sintering with $\sim 2$ at\% dopant concentration, and $\mathrm{Si}_{0.8} \mathrm{Ge}_{0.2}$ for the alloy. Two at $\%$ for Si-Ge is $\sim 1 * 10^{21} / \mathrm{cm}^{3}$, much higher than ideal for thermoelectrics, but dopant loss occurs during the laser sintering process and not all dopant is activated as the saturation limit is $2-3 * 10^{20} / \mathrm{cm}^{3}$. Therefore, starting with more dopant than desired in the final film is necessary.

The desired starting film thickness was 10-15 $\mu \mathrm{m}$. If the layer was too thick, bonding to the substrate could be difficult; however, this appears to be a function of the substrate material and thus further exploration may allow a wider acceptable window of film thicknesses.

Electrical resistance measurements were attempted on the raw films; however, the resistivity was never low enough to be measurable using the available 4 point probe.

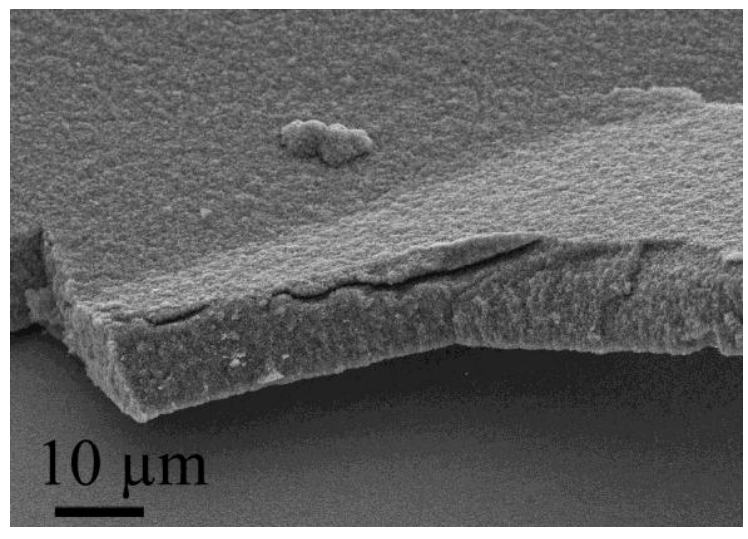

Figure 6.7. SEM image of $\sim 8 \mathrm{~nm}$ raw nanocluster film showing good morphology [48].

As a comparison to the nano-alloyed films, Figure 6.9 shows the alloying process of $\mathrm{Si}$ with Ge during ball milling. The Ge (111) and Si (111) peaks initially are located at $27.4^{\circ}$ and $28.5^{\circ}$, respectively, but after mechanical alloying the blue line shows how the peaks merge and for $\mathrm{Si}_{0.8} \mathrm{Ge}_{0.2}$ the peak moves to $\sim 28.3^{\circ}$ [143]. The (111) peak width for the nanoclusters is also wider than the mechanically alloyed case, indicated smaller crystallite size, which is a major benefit for achieving thermal conductivity values near the amorphous limit. 


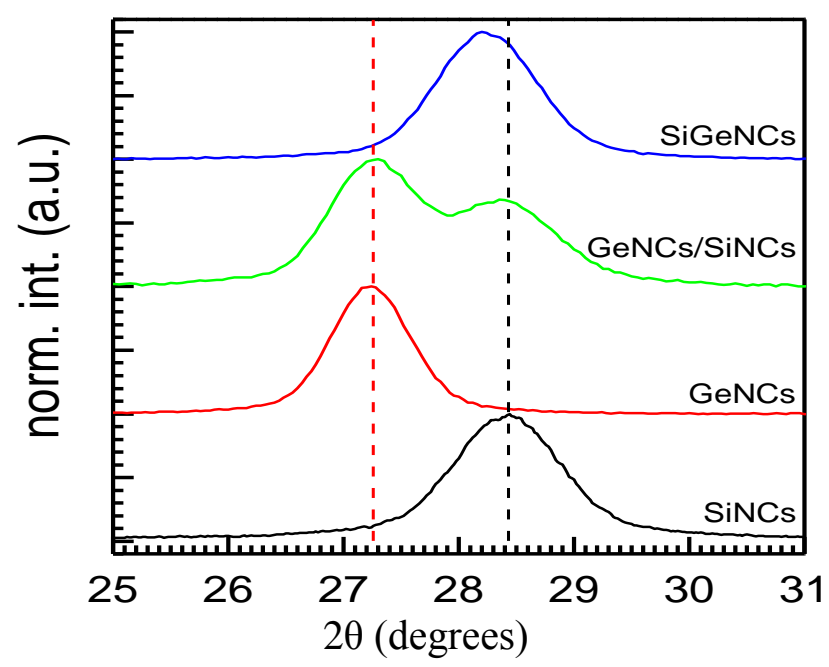

Figure 6.8. XRD of four types of film deposition: $\mathrm{Si}, \mathrm{Ge}$, mixed $\mathrm{Si}+\mathrm{Ge}$, and alloyed $\mathrm{Si}-\mathrm{Ge}$ [48].

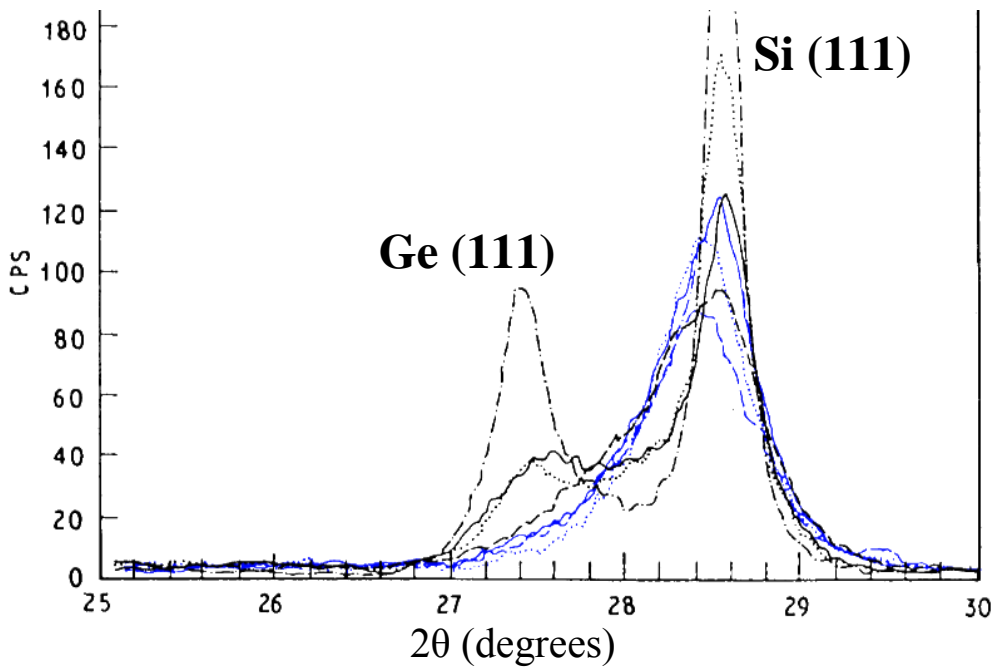

Figure 6.9. XRD study on mechanical alloying of $\mathrm{Si}$ and $\mathrm{Ge}$ to form $\mathrm{Si} 0.8 \mathrm{Ge} 0.2$ [143].

\subsubsection{Summary}

Two types of nanoparticles were used for laser sintering. The purchased nanopowders were undoped and difficult to deposit onto a substrate in a thin layer with low thickness

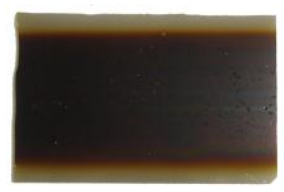

Figure 6.10. Doped

Si-Ge nanocluster film by UMN. variation. On the other hand, the doped nanoclusters from UMN solved these barriers, and after a few iterations to reduce film variation, the deposited films facilitated repeatable conditions to allow a more scientific study of laser sintering parameters. Such a film on aluminum nitride substrate is shown actual size in Figure 6.10. 


\subsection{Preheating using the Induction Heater (I.H.)}

The induction heater provides a valuable source of temperature adjustability during laser sintering, whether just to reduce thermal shock, or to assist in the sintering mechanism. For the nanocluster films, the hydrogen passivation could inhibit the sintering process. According to $\mathrm{UMN}, \mathrm{SiH}_{3}$ tends to decompose near $\sim 250^{\circ} \mathrm{C}$, though its concentration is low. More significantly, the $\mathrm{SiH}_{2}$ and $\mathrm{SiH}$ need to be heated to the $400-700{ }^{\circ} \mathrm{C}$ range to dissociate the hydrogen [144]. Thus, the films were annealed to $\sim 600^{\circ} \mathrm{C}$ for $60-150$ seconds before beginning laser treatment to ensure that the hydrogen bonds were broken. Similarly, Scheller et al. performed an anneal at $450{ }^{\circ} \mathrm{C}$ before pulsed laser annealing on Si-Ge nanoparticles to reduce the hydrogen content from their amorphous films [103]. Figure 6.11 shows the morphology of 5 nm Si-Ge nanoclusters after 5 minutes of heating with the I.H. set to 200 amps. The sample begins the process of necking and even grain growth appears to occur, but from XRD results (shown later in Figure 6.29) the crystallite size does not increase during the short duration of preheating at this setting.

Under the right conditions the reactivity of the sample surface could cause the surface to ignite, where the layer would essentially flashover, perhaps due to too much free oxygen or otherwise hydrogen outgassing during laser sintering. Figure 6.12 shows an SEM image where the ignition was occurring every $\sim 400 \mu \mathrm{m}$ along the laser scan line, distinctly changing the morphology and preventing the layer from being electrically conductive. The curved edges in the image are from the trailing edge of the laser beam, and they overlap in the direction of the scan path. This phenomenon was not noticed in later UMN sample batches, so either the preheating conditions were optimized or the film deposition quality was improved to mitigate the problem. 


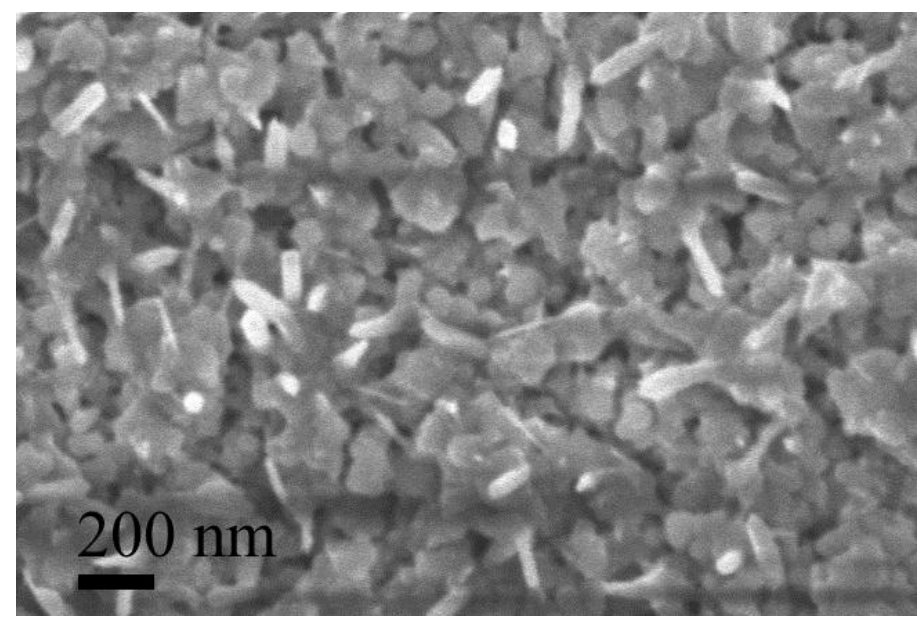

Figure 6.11. SEM image of $5 \mathrm{~nm} \mathrm{Si-Ge} \mathrm{nanoclusters} \mathrm{after} 5 \mathrm{~min}$. of the I.H. at $200 \mathrm{~A}\left(\sim 600^{\circ} \mathrm{C}\right)$.

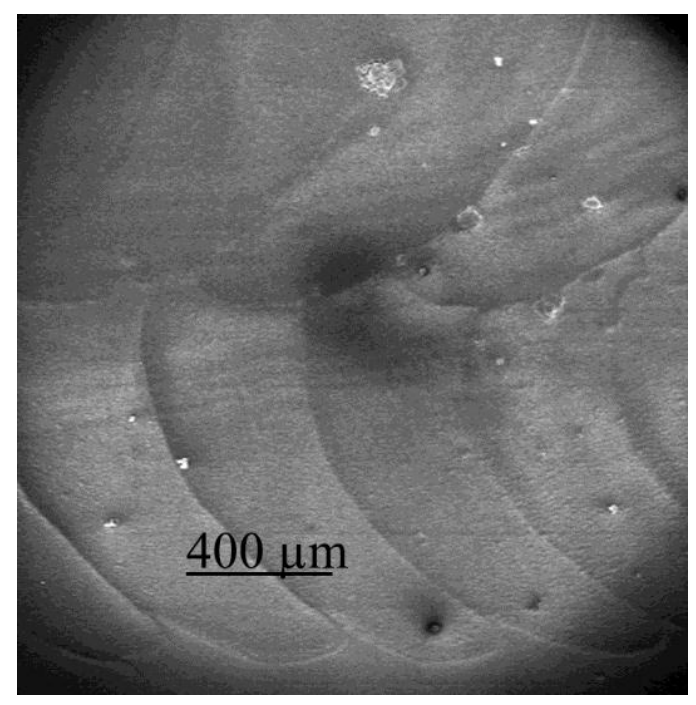

Figure 6.12. SEM image of Si-Ge nanocluster sintered film after laser-induced ignition.

One point of interest is that the magnetic field of the induction heater was strong enough to generate some changes in morphology on some samples, due to a mechanism such as acoustic waves or Hall effect. Figure 6.13 shows an example of how the Si-Ge nanoclusters were rearranged, including to curve around surface defects. If this effect occurred due to the applied magnetic field, the morphology change implies that the doped nanocluster films have at least slight micro-scale electrical conductivity as doped $\mathrm{Si}-\mathrm{Ge}$ is not considered a magnetically responsive material. 

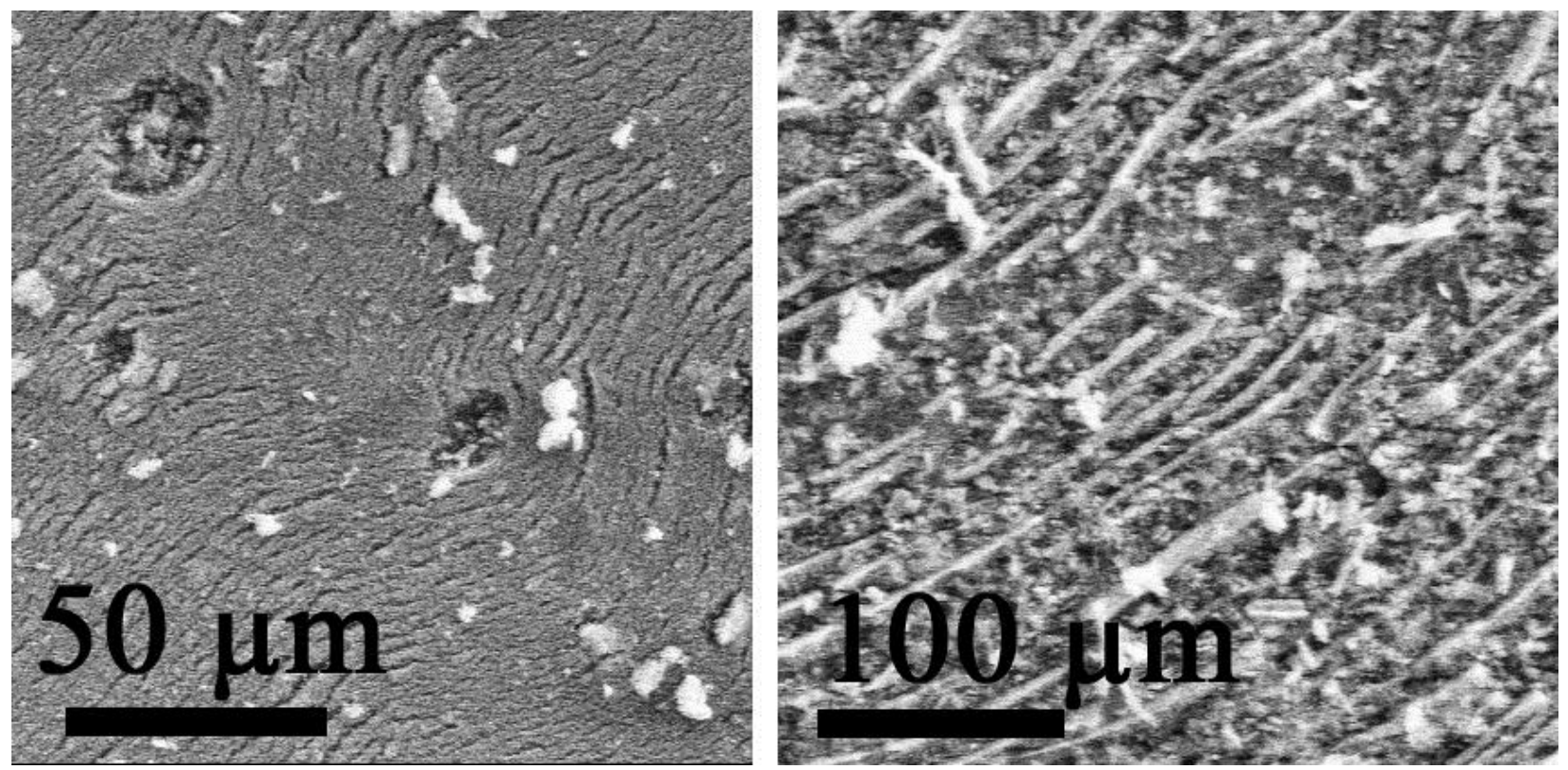

Figure 6.13. SEM images of induction heated Si-Ge nanoclusters showing surface effects.

\subsection{Sintering Results of Si-Ge Thin Films Using 940 nm CW Laser}

\subsubsection{Process Parameters}

Proper laser sintering conditions depend on many variables, many of which were listed in section 4.4. Two important factors are the size of the nanoparticles and matching the material composition with a laser wavelength with sufficient penetration. The reduced melting point of nanoclusters plays a role in the proper choice of preheating temperature and laser parameters, as the melting point can be $<50 \%$ of bulk [102]. Likewise, the absorption depth of laser energy of Si-Ge changes significantly for $532 \mathrm{~nm}$ vs. $940 \mathrm{~nm}$ wavelength lasers, especially for higher Ge concentrations. At $940 \mathrm{~nm}, \mathrm{Si}$ has an absorption depth of $\sim 55 \mu \mathrm{m}$ whereas Ge is $\sim 0.4 \mu \mathrm{m}$.

Figure 6.14 graphs the dependence of absorption depth on wavelength for silicon and germanium. As light absorption is dependent on the bandgap energy, higher temperatures and larger Ge concentration decrease the absorption depth, thus higher preheating temperatures tend to focus laser heat nearer to the thin film surface. 


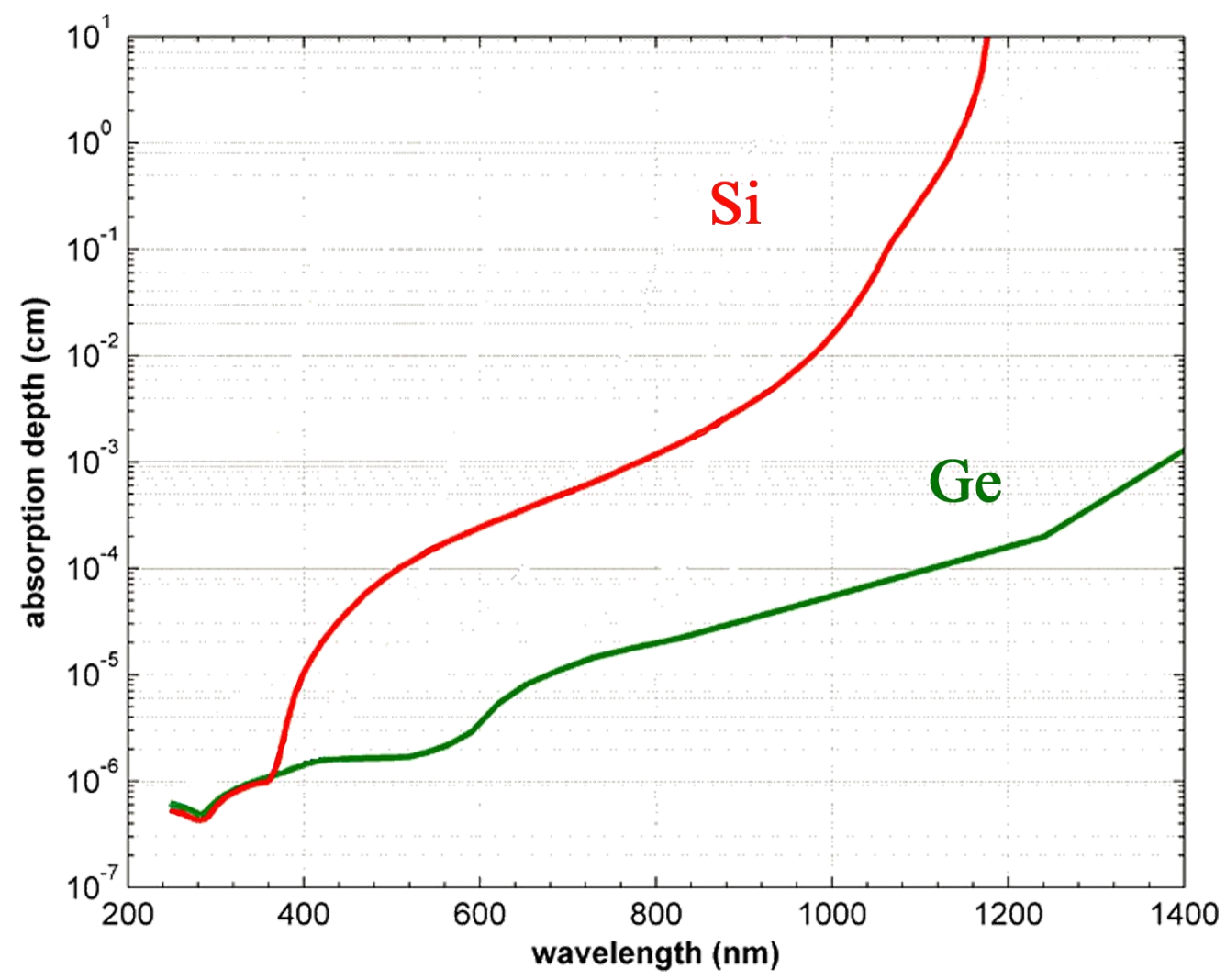

Figure 6.14. Absorption depth of light in silicon and germanium [145].

Significant obstacles for good-quality laser sintering results include:

a) doping which is well-dispersed at the nano-level

b) oxidation before and during laser processing

c) evaporation of dopants while sintering $\mathrm{Si}-\mathrm{Ge}$

d) maintaining a smooth sample surface

e) achieving complete densification and integral bonding with an inert substrate

Process parameters are chosen to achieve a good quality sintered film, as otherwise electrical conductivity suffers and electrical and thermal characterization may not be possible.

a) Having the dopant well-dispersed is important for laser sintering because the time-scale of heating is relatively short for allowing dopant diffusion in a non-molten state through a tremendous density of grain interfaces. The laser heating process can be balanced with the preheating, whereby a temperature just at the boundary of causing grain growth can facilitate 
laser beam densification and interconnection of the nanoclusters. Ultimately, some grain growth is necessary in order to achieve good density, but a balance must be found.

b) Oxide shells on the particles will likewise diminish electrical conduction and carrier mobility, so any heating must be done while the sample is free of oxygen and water vapor. Pulling vacuum in the chamber to reduce residual levels of gases, and then flushing argon through the chamber for at least one minute before beginning preheating, helped to reduce the side effects of oxidation.

c) In a further effort to reduce oxidation from the environment, and to help retain dopant vapors, a fused silica cover was used on the majority of laser sintered samples. Afterwards, when operating at higher laser intensities the coverplate has an optically visible film deposited on the side touching the sample, thus it certainly helps to maintain a higher local vapor pressure which will mitigate further losses.

d \& e) The last two issues go hand-in-hand. Under certain conditions with CW laser sintering of $\mathrm{Si}-\mathrm{Ge}$, as the nanoclusters begin necking and densification the surface tension pulls the individual clusters together. If the surface below is not simultaneously being wet by the particles then the clusters turn into spheres, absorbing any nearby particles but still maintaining the shape of a ball to minimize surface energy. These spheres contribute nothing to the electrical conductivity, and in fact make it harder to get a good contact with electrical probes, thus this morphology is to be avoided. At lower laser power levels and/or scan speeds, the semi-viscous $\mathrm{Si}-\mathrm{Ge}$ layer has a chance to percolate and smooth out without being driven to ball up. Yet, in this case adhesion to substrates such as fused silica is very difficult. The amount of scan line overlap also affects continuity in the direction perpendicular to the scan lines. While it may be impossible to make a perfect transition between scan lines, the morphology can be nearly as good as the center of the beam if the beam is properly focused and the overlap percentage correlates well with the operating power. For example, at lower intensities more overlap is needed because not all of the Gaussian beam will sinter the film; thus, if $70 \%$ of the beam width causes sintering, then a good starting point for overlap is $30 \%$. The overlap is specified in terms of line spacing, here referred to as linestep. The linestep parameter is the step distance between adjacent laser scan lines. 
On the other hand, at high laser intensities the energy is sufficient to assist wetting of the substrate, but this often happens near the limit of the mechanical and/or chemical stability of the substrate. Again, a balance must be found between densifying and ablating the film.

For the $940 \mathrm{~nm} \mathrm{CW}$ diode laser used most extensively in this research, the following parameters tended to produce the best results, or exceed the material limit. The samples were placed near the laser beam focal length for all results presented here.

Table F - Si-Ge nanocluster laser sintering parameters for various substrates.

\begin{tabular}{|l|l|c|c|c|c|c|c|}
\hline ID & Substrate & Condition & Intensity & I.H. & Speed & Linestep & Sample \\
\hline & & & $\mathrm{W} / \mathrm{mm}^{2}$ & $\mathrm{amps}$ & $\mathrm{mm} / \mathrm{s}$ & $\mathrm{mm}$ & \\
\hline$A$ & Fused silica & Continuity, no adhesion & 7 & 200 & 6 & 0.65 & SiGe6-14b1 \\
\hline$B$ & Fused silica & Some adhesion & 11 & 200 & 3 & 0.35 & SiGe5-8-1-c \\
\hline$C$ & Silicon & Dilution threshold & 46 & 250 & 8 & 0.48 & SiGe6-21a3 \\
\hline$D$ & Silicon & Substrate melting & 79 & 125 & 12 & 0.4 & SiGe6-22a1 \\
\hline$E$ & Silicon carbide & Percolation & 123 & 250 & 6 & 0.5 & SiGe27b3 \\
\hline$F$ & Silicon carbide & Wetting & 147 & 300 & 4 & 0.55 & SiGe26a6 \\
\hline$G$ & Silicon carbide & Substrate cracking & 130 & 250 & 6 & 0.52 & SiGe26b4 \\
\hline
\end{tabular}

Table $\mathbf{G}$ - Si-Ge sintering parameters for samples on silicon characterized to high temperature.

\begin{tabular}{|c|c|c|c|c|c|c|c|}
\hline Sample & Scan & I.H. & Linestep & Speed & Intensity & Fluence & Resistivity \\
\hline & directions & amps & $\mathrm{mm}$ & $\mathrm{mm} / \mathrm{s}$ & $\mathrm{W} / \mathrm{mm}^{2}$ & $\mathrm{~J} / \mathrm{mm}^{2}$ & $\mathrm{~m} \Omega \cdot \mathrm{cm}$ \\
\hline 21a1 & $\mathrm{X}$ & 200 & 0.48 & 8 & 57.1 & 6.0 & 6.3 \\
\hline 21a3 & $\mathrm{X}$ & 250 & 0.48 & 8 & 46.1 & 4.8 & 4.9 \\
\hline 21b1 & $\mathrm{X}$ & 200 & 0.48 & 12 & 62.7 & 4.4 & 12 \\
\hline 21b2 & $\mathrm{X}$ & 200 & 0.6 & 2 & 46.1 & 19.3 & 5.4 \\
\hline 21b3 & $\mathrm{X}$ & 250 & 0.48 & 12 & 53.0 & 3.7 & 4 \\
\hline 21b4 & $\mathrm{X}$ & 200 & 0.48 & 8 & 57.1 & 6.0 & 4.9 \\
\hline 21e2 & $\mathrm{XY}$ & 200 & 0.75 & 4 & 51.6 & 10.8 & 4.7 \\
\hline 21e5 & $\mathrm{X}$ & 200 & 0.48 & 8 & 57.1 & 6.0 & - \\
\hline 21e6 & $\mathrm{XY}$ & 150 & 0.45 & 12 & 62.7 & 4.4 & 4.1 \\
\hline 22a4 & $\mathrm{XY}$ & 200 & 0.7 & 4 & 51.6 & 10.8 & 44 \\
\hline 22a5 & $\mathrm{X}$ & 200 & 0.48 & 8 & 57.1 & 6.0 & 25 \\
\hline 22c6 & $\mathrm{X}$ & 250 & 0.4 & 12 & 51.6 & 3.6 & 14 \\
\hline 22d4 & $\mathrm{X}$ & 250 & 0.4 & 12 & 53.0 & 3.7 & 22 \\
\hline
\end{tabular}

The laser processing conditions in Table $\mathbf{G}$ provide an overview of the conditions used for samples which were analyzed to high temperature. All conditions are sufficient for the nanoclusters to wet the Si substrate surface, and although the scan speed and fluence cover relatively large ranges of 46 to $63 \mathrm{~W} / \mathrm{mm}^{2}$ and 3.6 to $19 \mathrm{~J} / \mathrm{mm}^{2}$, respectively, the balance of parameters such as preheating and scan speed allow similar sintering quality and help to define the process window for cladding the silicon substrate with nanoclusters. The induction heating 
range was about $500{ }^{\circ} \mathrm{C}$ to $800{ }^{\circ} \mathrm{C}$ for the 150 to 250 amps range. Owing to the high thermal conductivity of the substrate, the fluence is not a critical parameter like it would be with a pulsed laser.

\subsubsection{Morphology and Composition}

Under the proper processing conditions, the laser sintered samples have good morphology in terms of density and continuity, and have achieved adhesion to the substrates. These results show that $\mathrm{Si}-\mathrm{Ge}$ nanoparticles can be successfully laser sintered into a continuous film.

When not providing sufficient preheating and/or laser intensity, the nanoclusters begin agglomeration but do not reach a sufficient temperature to fully sinter, as shown in the SEM image in Figure 6.15. Process parameters were no preheating and $9 \mathrm{~W}$ of laser power. The low level of heat does not drive mass transfer strongly enough to pull the segregated clusters together for good densification.

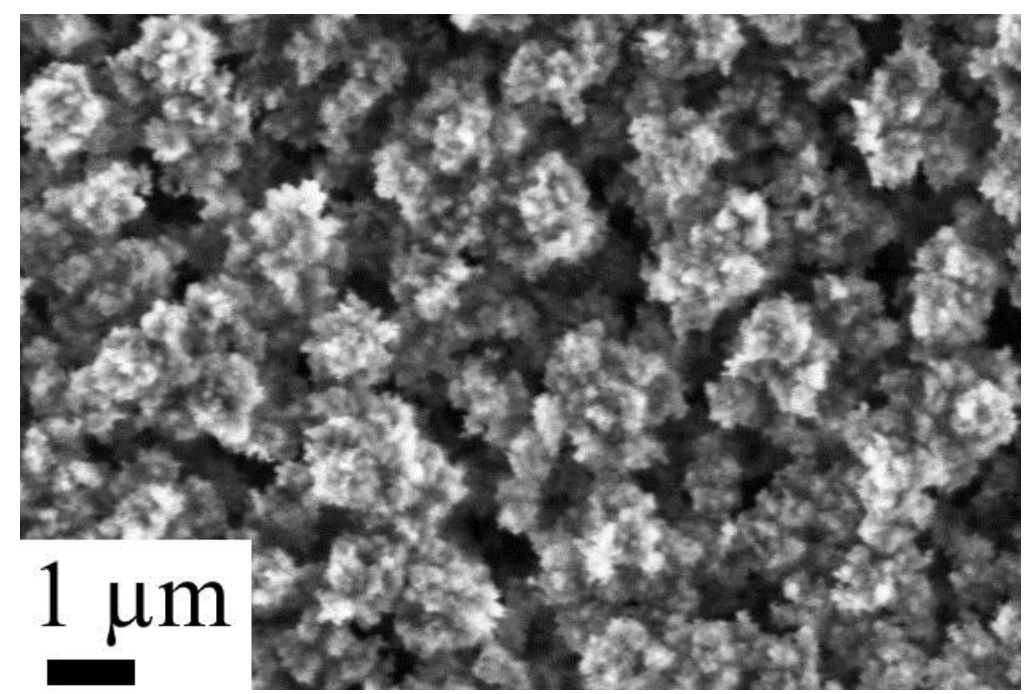

Figure 6.15. SEM image of Si-Ge nanoclusters after only low power laser heating.

Figure 6.16 shows the transformation from the raw black non-reflective nanoclusters into a silvery metallic reflective film after laser sintering $(A)$. This condition was achieved with a laser intensity just below the onset of balling, achieving good continuity but not significant adhesion to the substrate. 


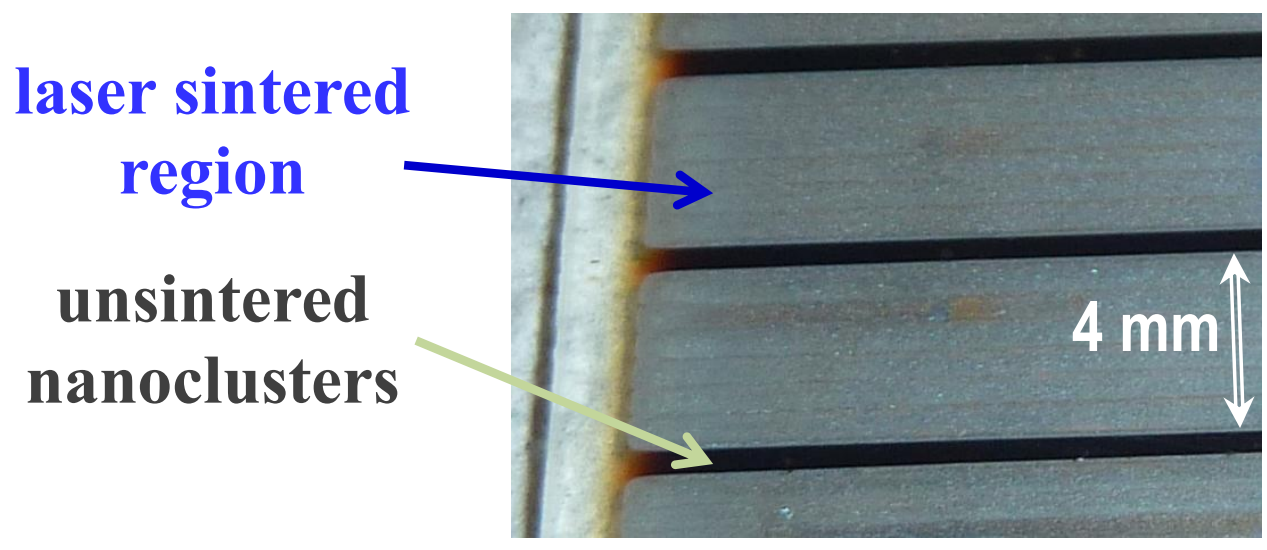

Figure 6.16. Visible image of laser sintered nanocluster film showing high reflectivity.

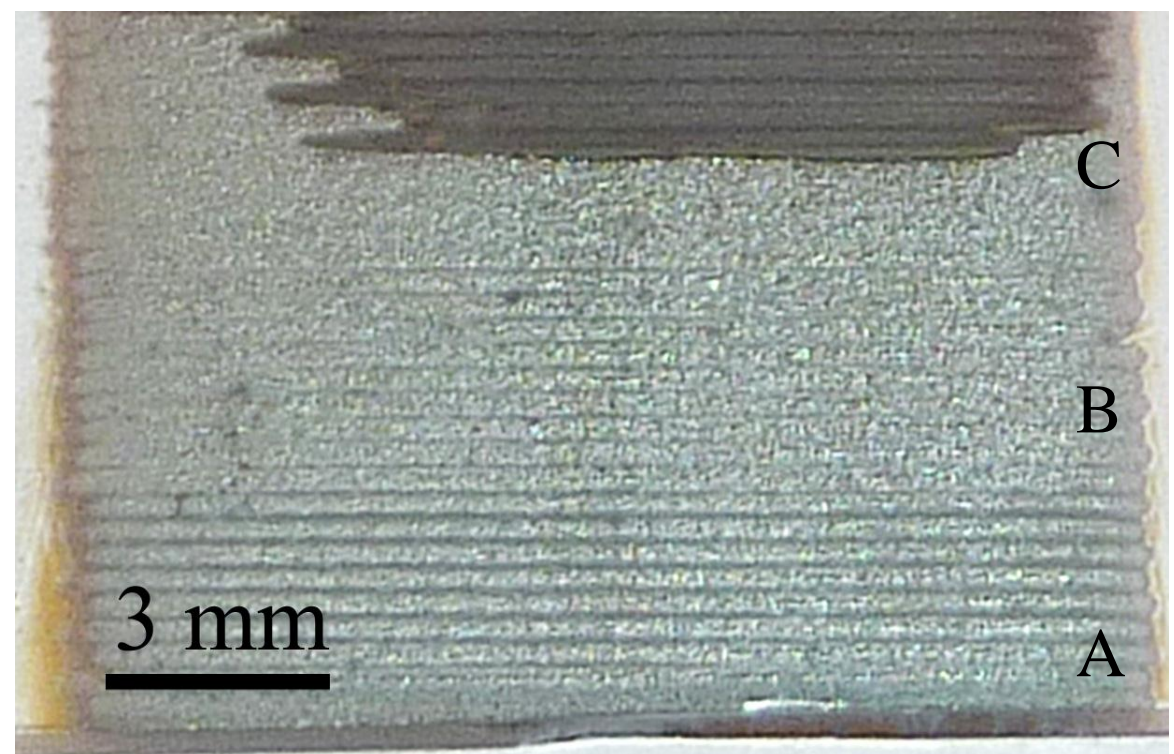

Figure 6.17. Visible image of sintered nanocluster film on silica comparing scan line overlap.

Figure 6.17 shows a similar example of sintering on fused silica substrate $(B)$, but in this case the sintered film adhered to the substrate, which facilitated characterization. This visible image shows the scan lines, and the effective sintering line-width of the laser beam at relatively low power levels. Given the $350 \mu \mathrm{m}$ step between scan lines, in area A the process conditions achieve a sintering beam width of $\sim 275 \mu \mathrm{m}$, in area $\mathrm{B} \sim 325 \mu \mathrm{m}$, and in area $\mathrm{C}>350 \mu \mathrm{m}$. The dark region in section $\mathrm{C}$ also exemplifies the transition from percolation to balling, where the surface roughness is much higher and thus the reflectivity decreases tremendously. Likewise, the conductivity in this region is very poor. 
Figure 6.18 shows an optical microscope (OM) image of a similar sample at 100x magnification. Note the scale bar is approximate. Here the surface morphology can be described as variable but reasonably smooth. The limited depth of field causes the highest peaks and deepest valleys to be blurred, but by adjusting the focus the peak to peak height was estimated as 5 to $10 \mu \mathrm{m}$. At the middle of the image is a beam overlap edge, where the $2^{\text {nd }}$ pass is shown at the bottom half of the image. The color shift occurs due to optical inference, resulting from vaporization and subsequent redeposition of a thin layer of Si-Ge. The surface roughness of the $1^{\text {st }}$ pass (image top) increases due to this redeposition, thus lacking the smoothness visible in the $2^{\text {nd }}$ pass (image bottom).

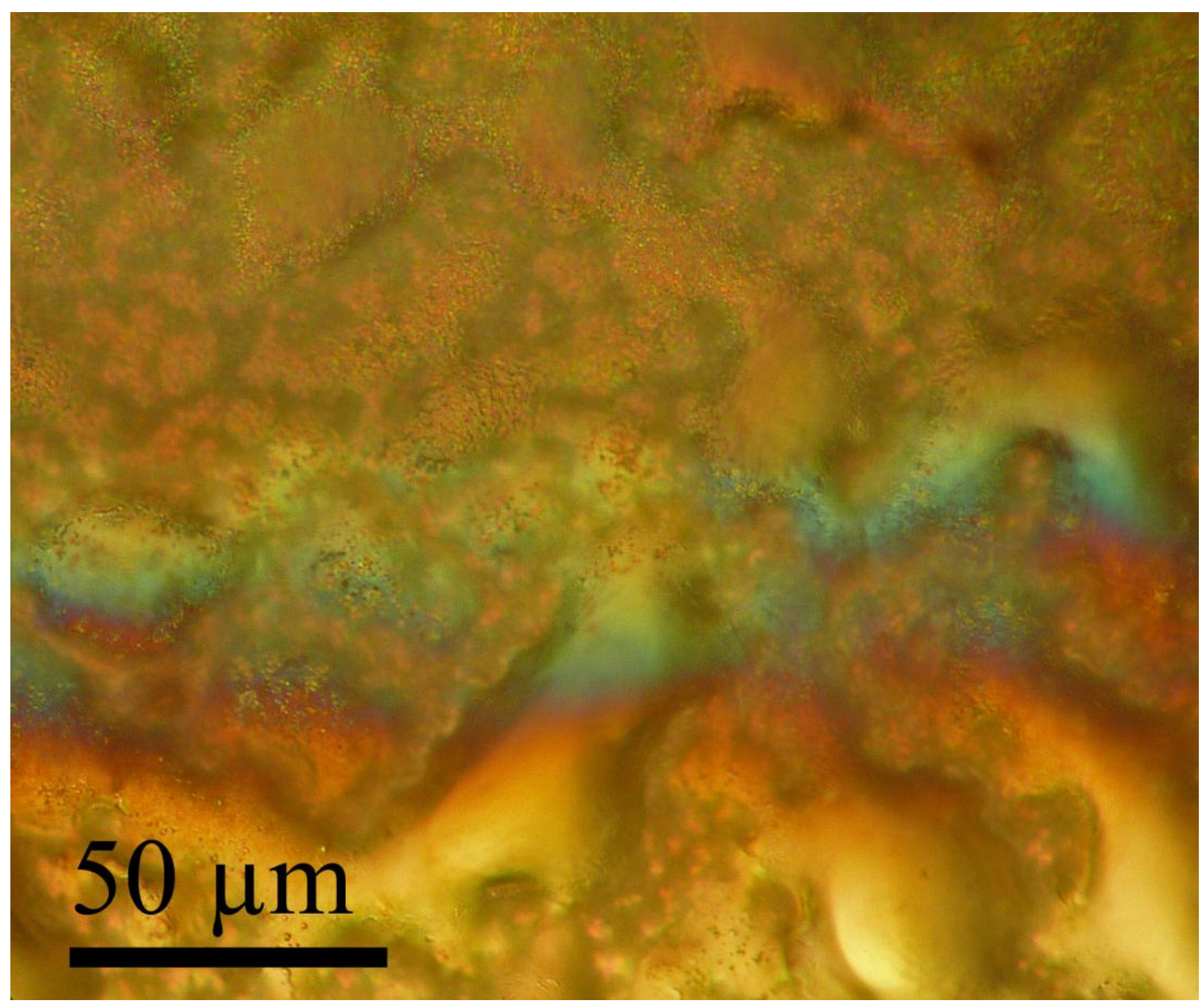

Figure 6.18. Optical image of laser sintered nanocluster film showing wetting. 


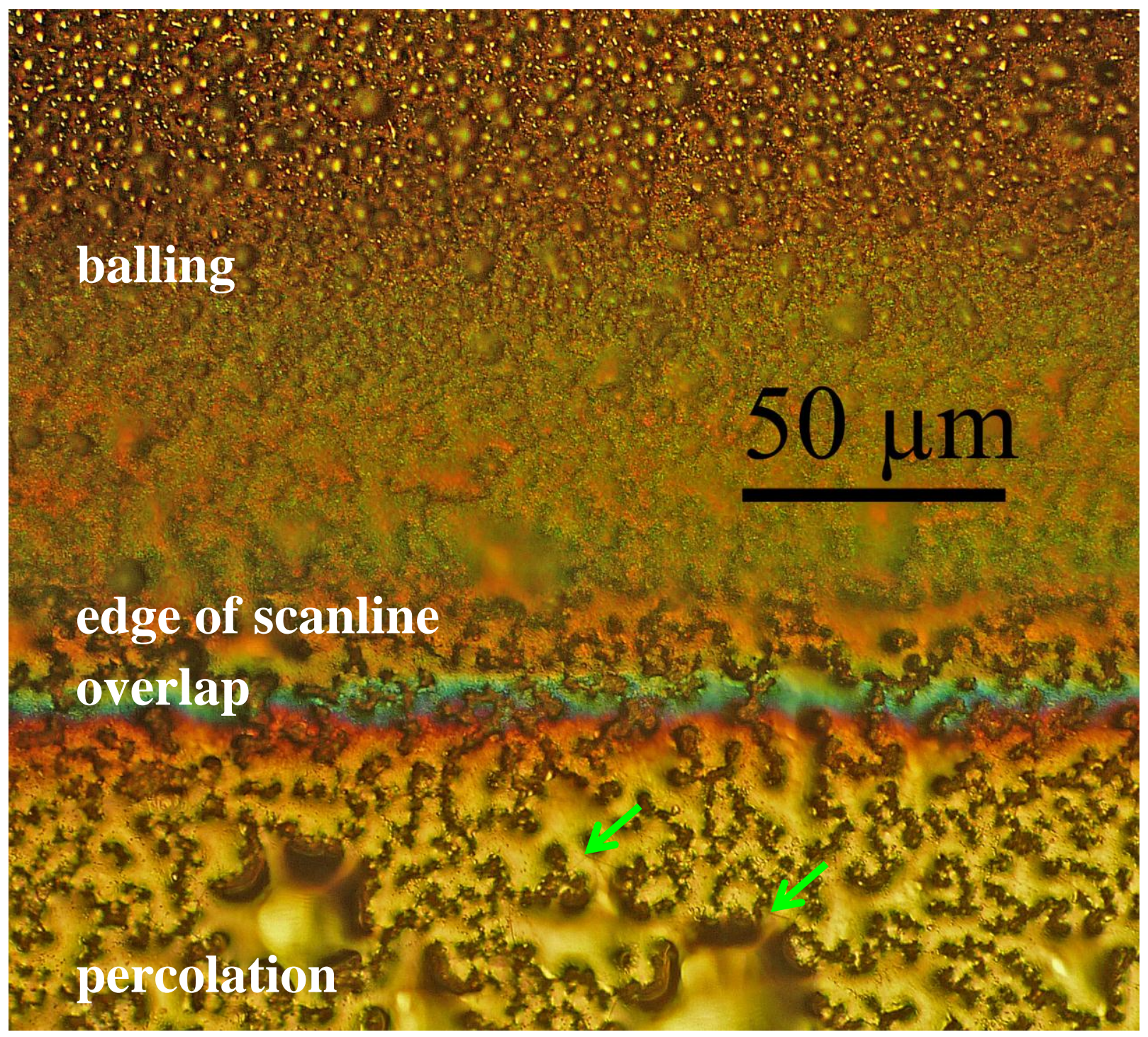

Figure 6.19. Optical image of laser sintered nanocluster film showing percolation and balling.

Figure 6.19 is an optical image at 100x of sample $B$, showing the overlap between two scan lines, the top one where balling has occurred and the bottom one in the percolation regime. Note in the percolating network there are also micro-cracks due to the thermal strain during cooling, as illustrated by the arrows. Figure 6.20 shows two SEM images comparing the morphologies of the two regions in Figure 6.17, percolating (shiny) vs. balling (dark). A thin-film percolating network is suggested to reduce in-plane macroscopic electrical conductivity by approximately one order of magnitude compared to bulk material [107]. The conductivity of the balled region, however, is many orders of magnitude lower than the percolating region. 

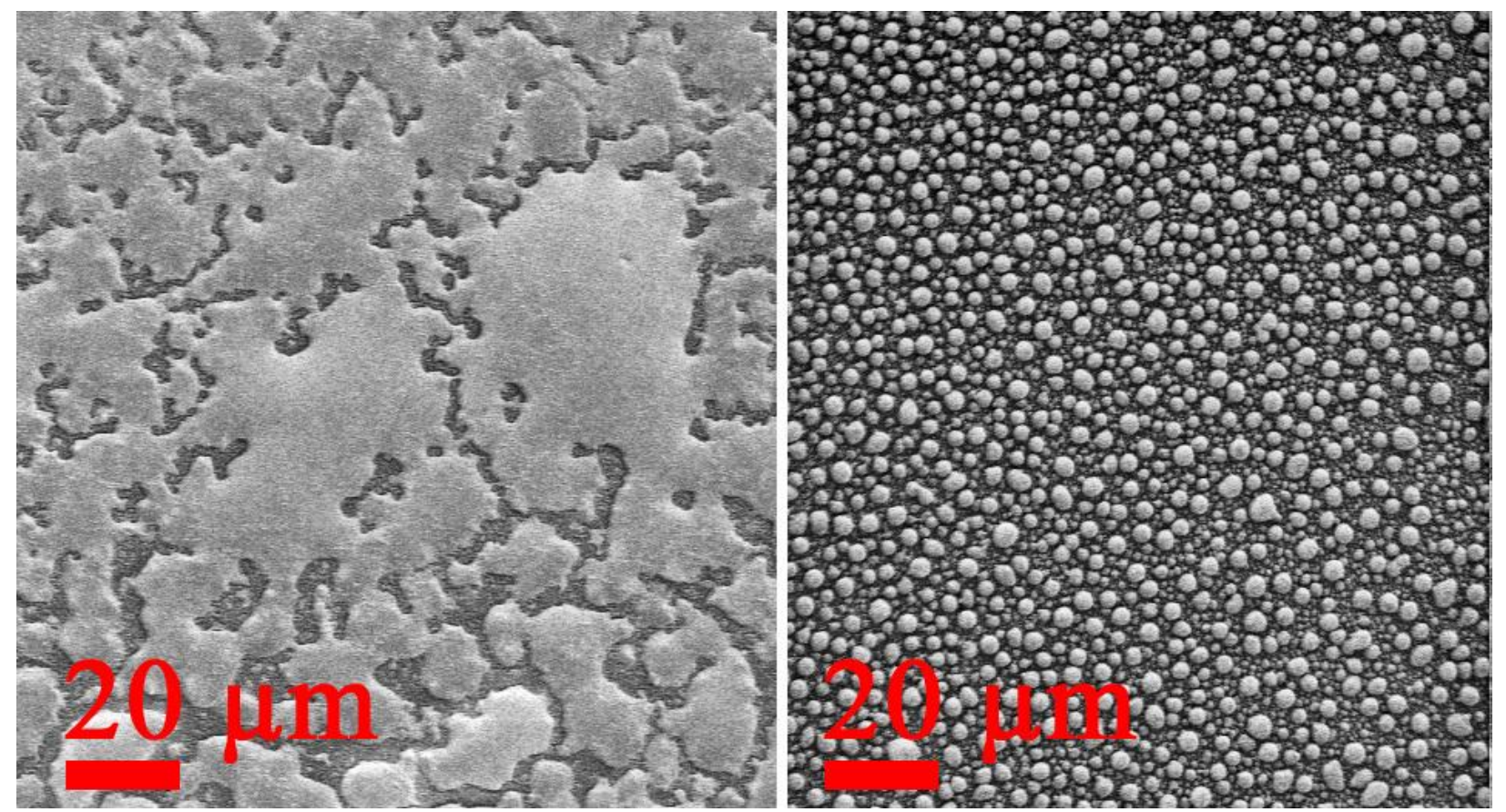

Figure 6.20. SEM image of comparing morphologies of percolation and balling.

Figure 6.21 is a tilted SEM image of the interface between the sintered percolating film and the fused silica substrate after cleaving the substrate to view the cross-section. The fact that the film did not delaminate during the cleaving process proves that it is well-bonded to the substrate.

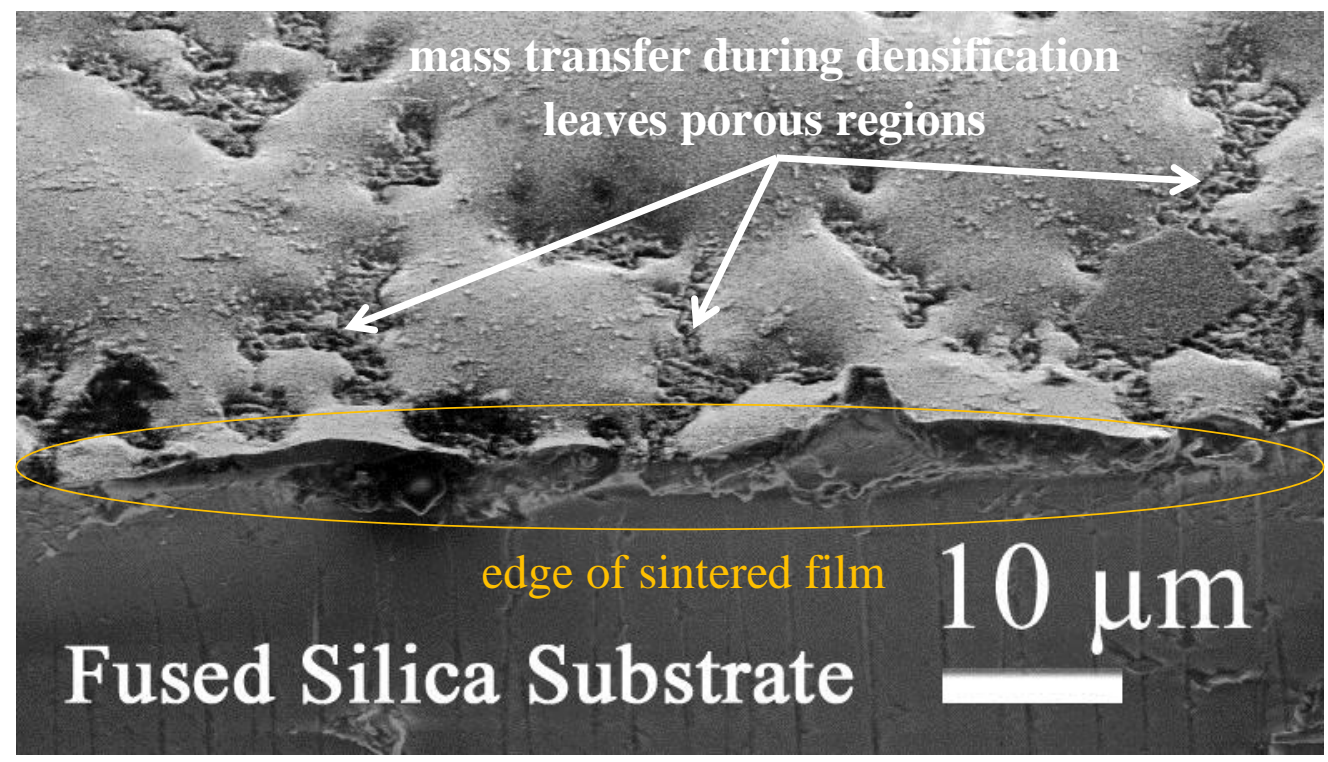

Figure 6.21. SEM image of comparing morphologies of percolation and balling. 
Figure 6.22 shows the surface morphology of a percolating network from low to high magnification. The laser sintered film has good continuity and overall macro-scale regularity. The white regions exemplify film porosity, due to mass transfer during particle densification resulting in sporadic voids. At the micro-scale, the surface has some inclusions, but has low roughness for an unpolished finish of laser sintering on a glass substrate. In the top left image of Figure 6.22, the laser beam was scanned in the x-axis and it's possible to distinguish two full laser scan lines. While the morphology has some variation in the y-axis due to the Gaussian laser beam profile and scan line overlap, the electrical conductivity is similar in both directions. The higher magnification images show the surface has a few larger grains, but primarily still retains nanofeatures after laser sintering.
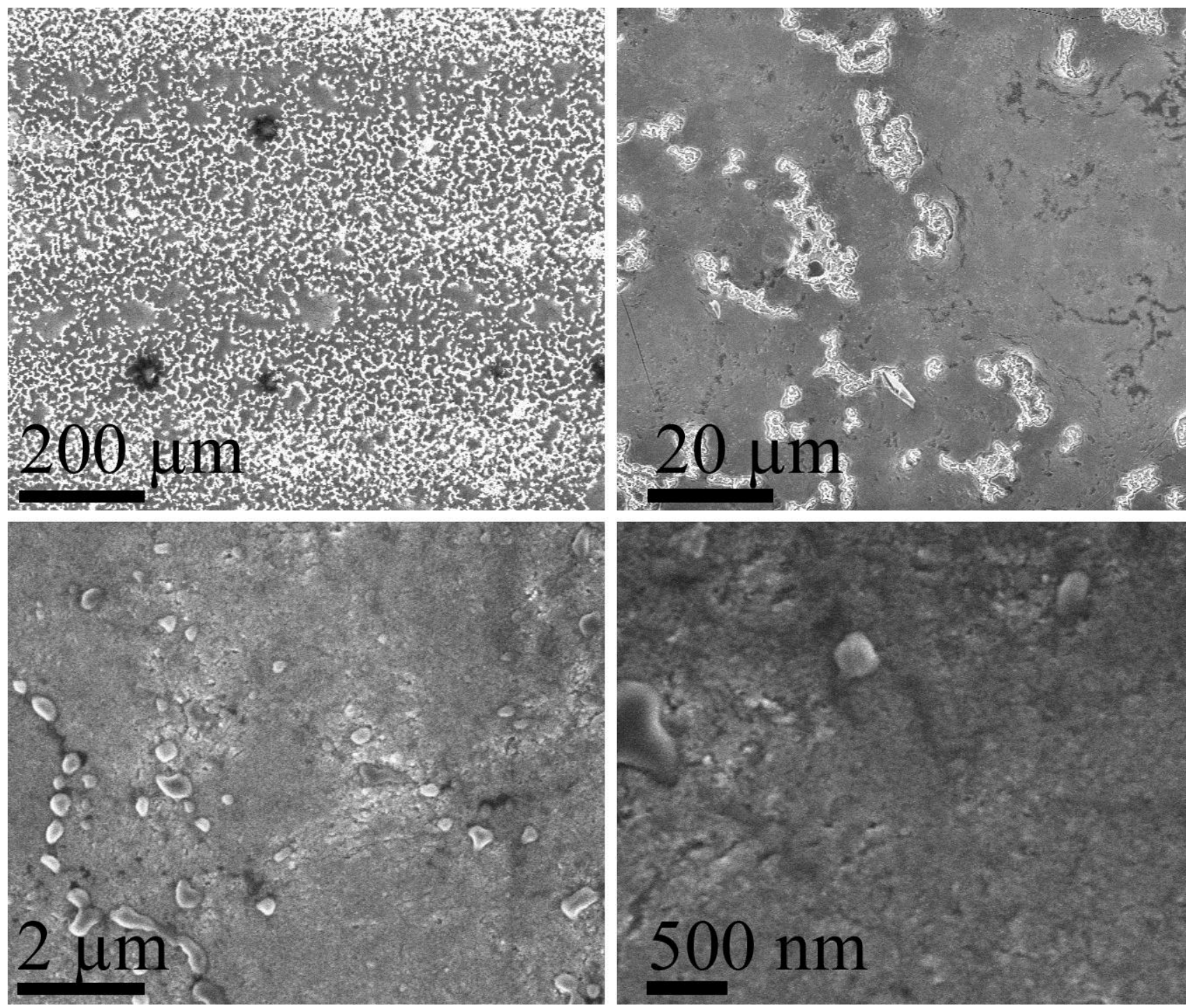

Figure 6.22. SEM image showing morphology of percolation at various scales. 
When balling occurs, it can also result in a significant loss of germanium. As the Ge preferentially absorbs the laser energy, if it is not well-alloyed with the Si it can segregate from the film surface into balls. Probing the films with EDS reveals that while the surface usually is reported as having 5 to 10 at\% Ge, the balls tend to be much higher, as in Figure 6.23 where the composition of an abnormally large ball of $\sim 14 \mu \mathrm{m}$ is $\mathrm{Si}_{0.2} \mathrm{Ge}_{0.8}$, using a $10 \mathrm{kV}$ accelerating voltage. It should be noted that the composition reported by Oxford's Inca software, when running without standards, varies significantly depending upon the accelerating voltage chosen. At $10 \mathrm{kV}$, the Ge concentration is somewhat overestimated.
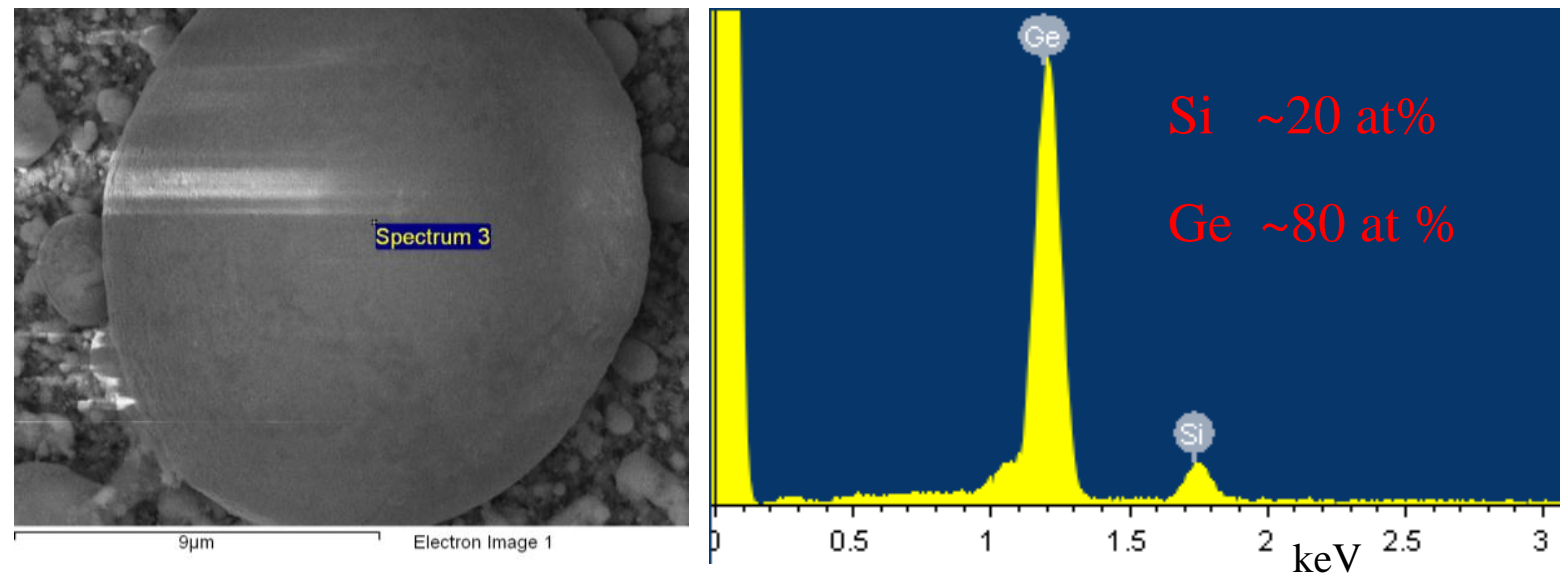

Figure 6.23. SEM/EDS of $\sim 10 \mu \mathrm{m}$ diameter ball, showing Ge segregation.

While balling presents a significant problem in terms of obtaining a continuous and conductive film, the issue can be mitigated by sintering on different substrates which are better wet by molten silicon and germanium. For instance, the SEM image in Figure 6.24 shows a cross-section of a nanocluster film sintered on silicon, where full integrity has been achieved at the bonding interface, to the point that even if remnants of balls remain, they will cleave in half when cleaving the substrate. Unfortunately, in this case the strong interface resulted from significant reaction between the film and Si substrate, whereby the dopant migrated tens of microns deep into the substrate. While this may have application elsewhere, to properly characterize thermoelectric performance the materials must be homogenous.

Figure 6.25 shows a top view of a laser sintered surface after polishing and deposition of a Ag thin-film transducer layer, the same sample as in Figure 6.22 before polishing. The nanofeatures of the surface are just resolvable, and porosity can be nearly zero in locations like this one. Figure 6.26 shows another sample cross-section, however this time the substrate is silicon 
carbide. The continuous regions of the film are $\sim 0.5 \mu \mathrm{m}$ thick, and the films do bond to the substrate but at quite high power levels.

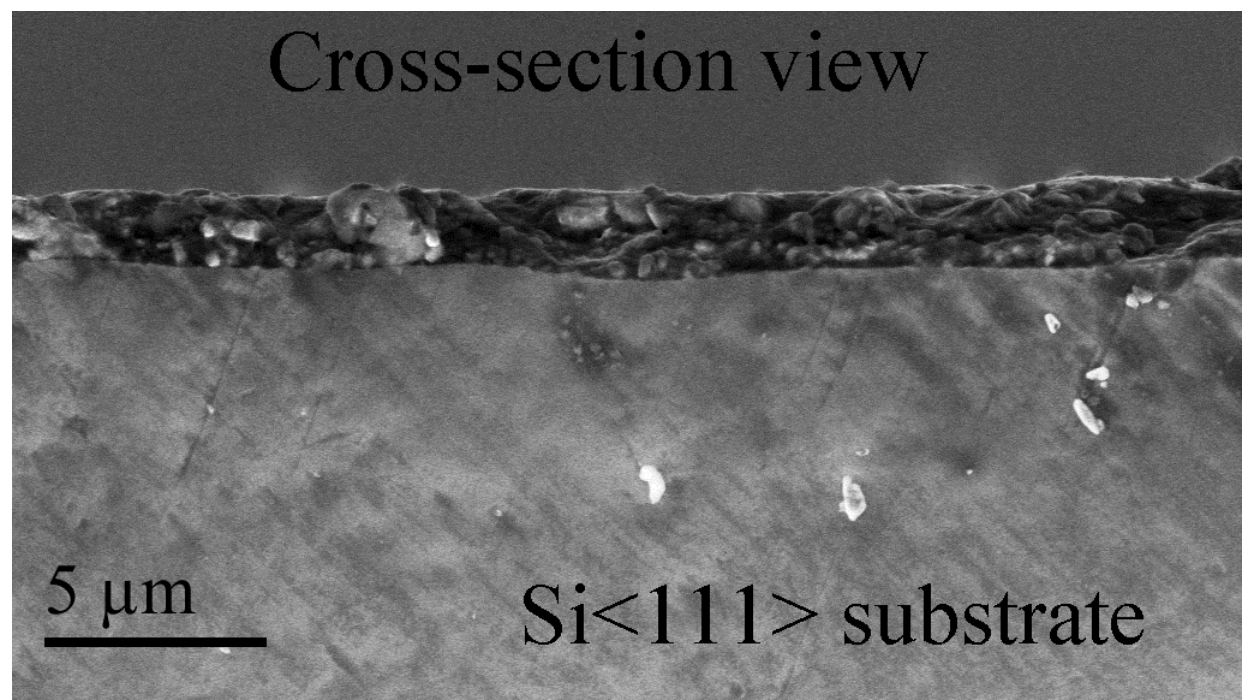

Figure 6.24. SEM of laser sintered nanocluster film showing densification and continuity.

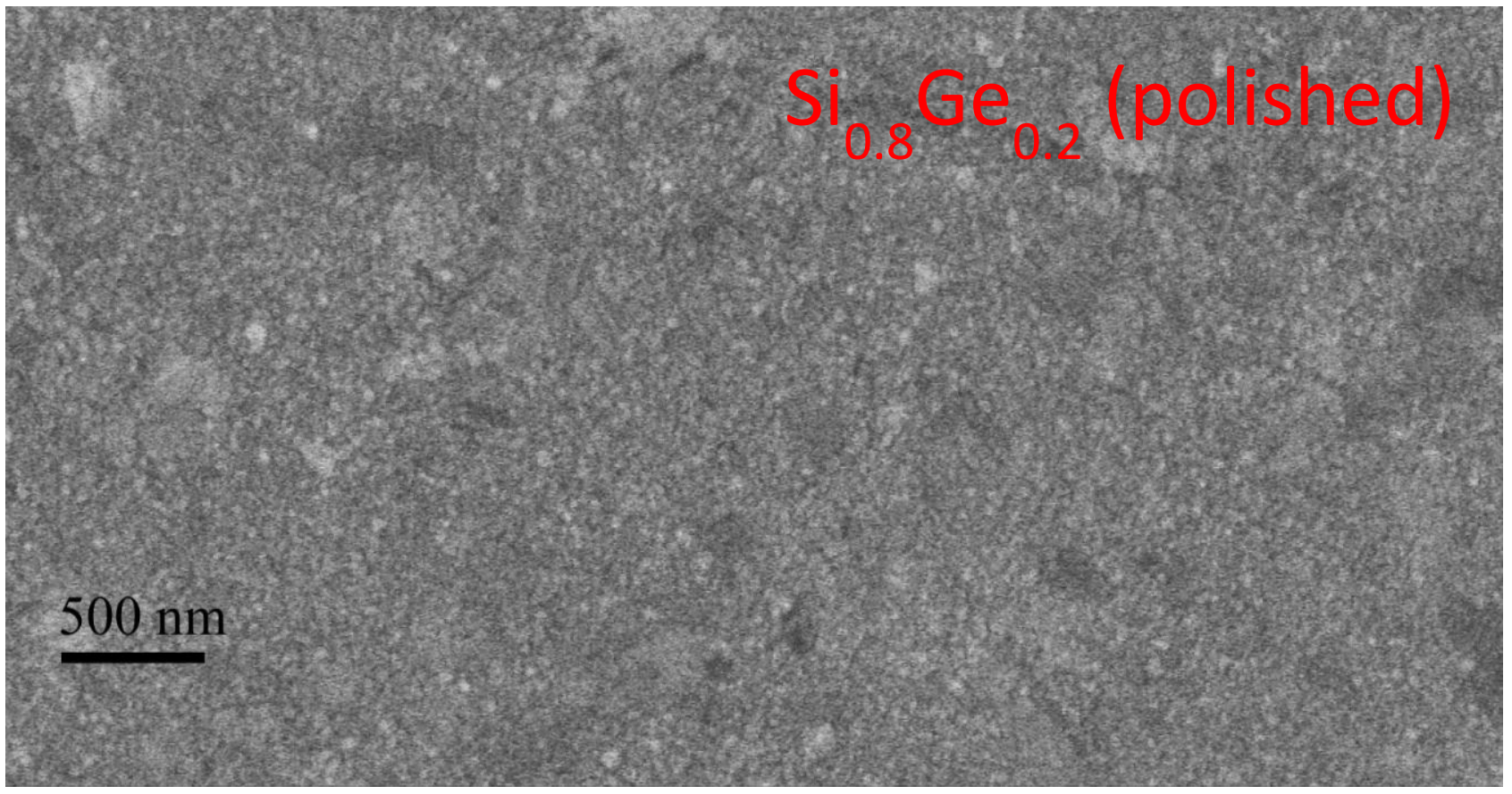

Figure 6.25. SEM of polished laser sintered surface used for TDTR showing nanograin features. 


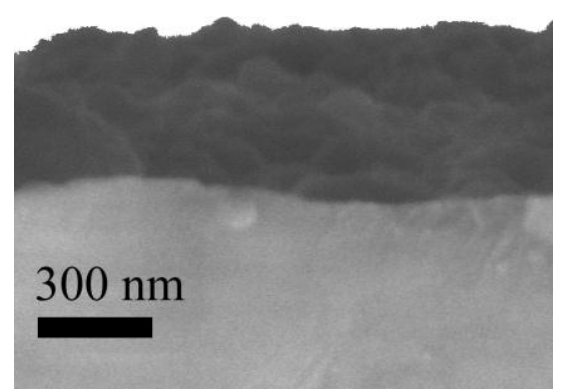

Figure 6.26. SEM of laser sintered nanocluster film on SiC showing densification and adhesion.

\subsubsection{Doping Depth}

As the laser sintering on Si substrates provided very low resistivity and a high Seebeck value, it became clear that the dopant diluted into the substrate during laser heating. To determine the effective conducting thickness, Reactive Ion Etching (RIE) was employed to etch the surface, and resistance measurements were taken with a four point probe after every $\sim 5 \mu \mathrm{m}$ of etching. Figure 6.27 displays the resistance measurements as a function of depth for four samples sintered under representative conditions, revealing that the conductive depth is $\sim 40 \mu \mathrm{m}$. Here the conductive depth is defined as the point where in-plane resistance has increased by a factor of 100 , leaving $1 \%$ error on the calculated average conductivity value.

The resistivity of 3 of the 4 samples quickly reaches intrinsic levels beyond $\sim 40 \mu \mathrm{m}$ depth. Using these resistance measurements, the resistivity of each $\sim 5 \mu \mathrm{m}$ layer could be determined by using a parallel resistor model. Furthermore, by using the carrier concentration vs. resistivity curves in Figure 5.6, the doping concentration vs. depth can be calculated. To compute carrier concentration from resistivity, the composition was assumed to be a homogeneous $\mathrm{Si}_{0.98} \mathrm{Ge}_{0.02}$. This estimation comes from the initial dose of $\mathrm{Ge}$ being the equivalent of $\sim 1 \mu \mathrm{m}$ thick, which dispersed over $\sim 40 \mu \mathrm{m}$ thickness gives $\sim 2 \%$ concentration; the computation is relatively insensitive to composition ( $7 \%$ lower for $\mathrm{Si}_{0.9} \mathrm{Ge}_{0.1}$ ). Although the data has considerable variation, a logarithmic fit of the carrier concentration gives all four samples as being between $7.2 * 10^{18}$ and $1.5^{*} 10^{19} / \mathrm{cm}^{3}$ at the surface. Three of the four samples measured have very similar slopes, and thus the average of those three is plotted in Figure 6.28. The average surface carrier concentration is $1 * 10^{19} / \mathrm{cm}^{3}$. The trend line shows that the carrier concentration decreases with depth, which is to be expected for diffusion-based doping. 


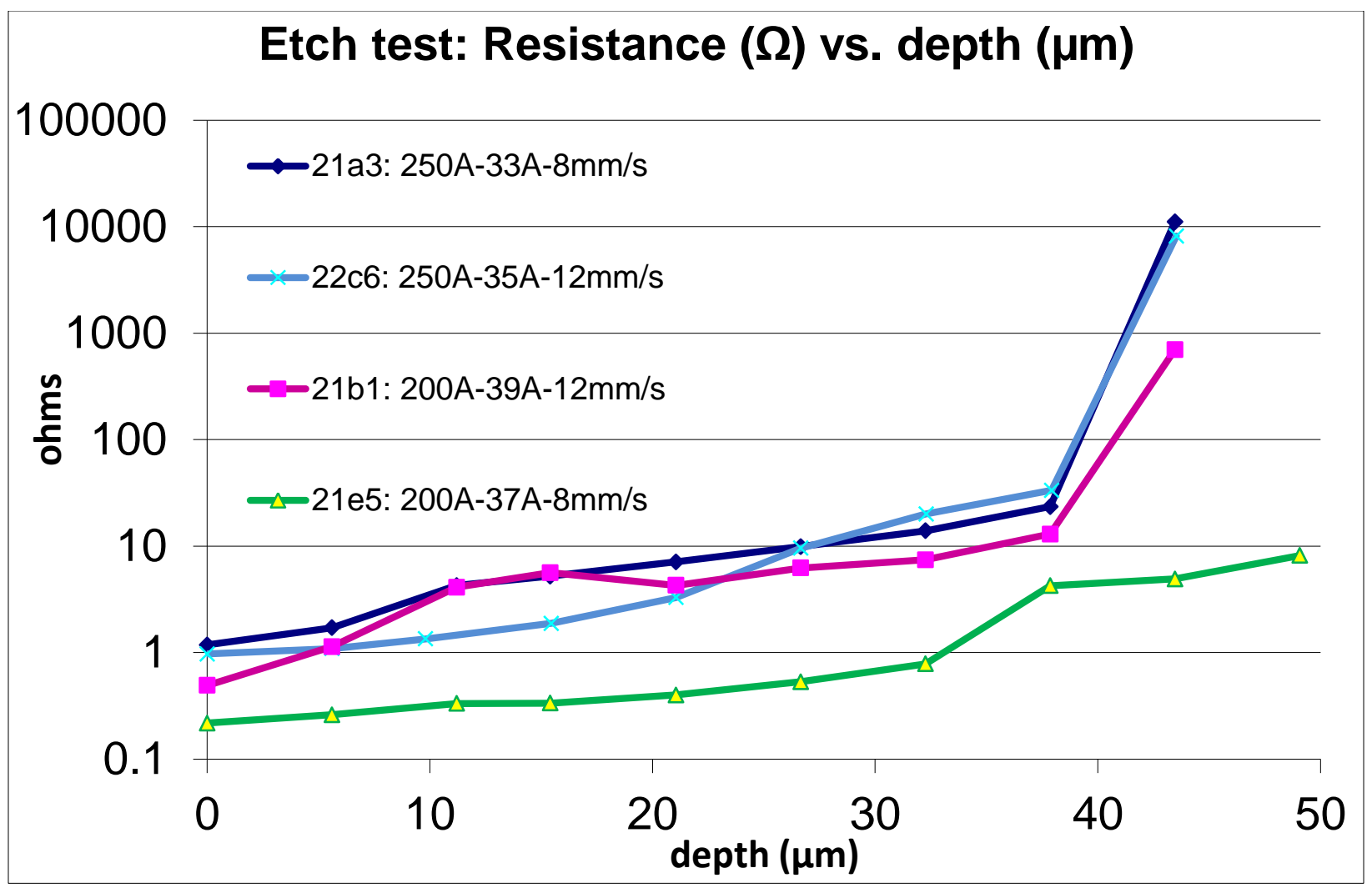

Figure 6.27. Resistance vs. depth for laser sintered samples on Si substrates.

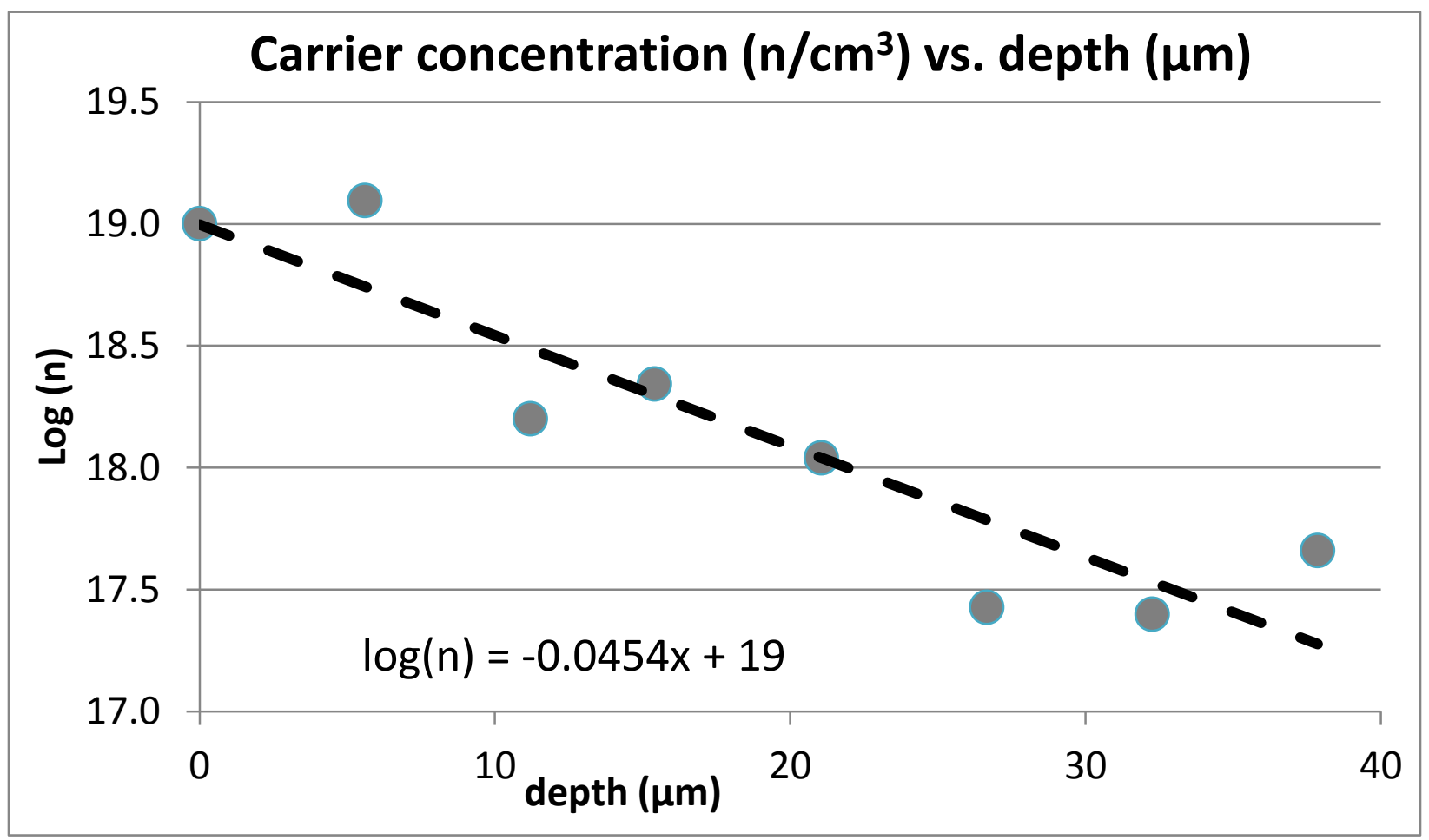

Figure 6.28. Trend of carrier concentration vs. depth for laser sintered samples on Si substrates. 


\subsubsection{Crystal Structure and Stability}

High temperature characterization of the $\mathrm{CW}$ laser sintered samples was performed at ORNL's High Temperature Materials Laboratory (HTML). All XRD scans were performed in an environment of $\mathrm{N}_{2}+4 \% \mathrm{H}_{2}$, at a scan rate of $\sim 1$ hour per temperature curve. To isolate the impact of solely pre-heating, one sample was characterized using high temperature XRD after the Si-Ge nanoparticles had been preheated for 60 seconds in the induction heater set to 200 amps by resting on a molybdenum substrate $\left(\sim 600^{\circ} \mathrm{C}\right)$. The XRD scans in Figure 6.29 showed significant grain growth between 500 and $600{ }^{\circ} \mathrm{C}$, implying that the sample temperature did not significantly exceed $600{ }^{\circ} \mathrm{C}$ during the induction heater preheating process. The broad background peak at $\sim 22^{\circ}$ resulted from the $\alpha$-quartz substrate. The sharper peak at $25.4^{\circ}$ in the low-temperature curves matches alumina, which is a major component of the Macor sample holder. As the sample annealed in the XRD, the signals from the substrate and holder were reduced, and the peaks from the silicon germanium alloy became much sharper. The three primary Si-Ge peaks were $27^{\circ}, 46^{\circ}$, and $54^{\circ}$, as remaining in the final room temperature scan. Initial crystallite size was $\sim 5 \mathrm{~nm}$, and after the XRD measurements the size exceeded the $\sim 100$ $\mathrm{nm}$ detection limit using XRD. Therefore, the grain size change during characterization proved that preheating did not cause grain growth and that the nanoclusters have a tremendously depressed melting point from the bulk value of $1275^{\circ} \mathrm{C}$.

Additional XRD scans of Si-Ge nanoclusters confirmed that later batches had a larger initial crystallite size of $9 \mathrm{~nm}$, rather than the original nanocluster crystallite size of $\sim 5 \mathrm{~nm}$. This nanocluster size change was implemented as it was felt that the huge surface area to volume ratio of the $5 \mathrm{~nm}$ particles increased the oxygen adsorption. The volume fraction of atoms in an oxide shell will continue to decrease as the particle diameter is increased, given a constant oxide shell thickness of $\sim 1-2 \mathrm{~nm}$. Figure 6.30 shows the XRD scans of three Si-Ge nanocluster films: Blue) unprocessed, Green) after 8 minutes of induction heating at 200 amps, RED) after 8 minutes of induction heating at $250 \mathrm{amps}$. The full-width half-maximum of peaks indicates the crystallite size is $9 \mathrm{~nm}$ for all three conditions, again confirming that preheating with up to $250 \mathrm{amps}$ on the induction heater does not cause grain growth within the time required to laser sinter the films. 


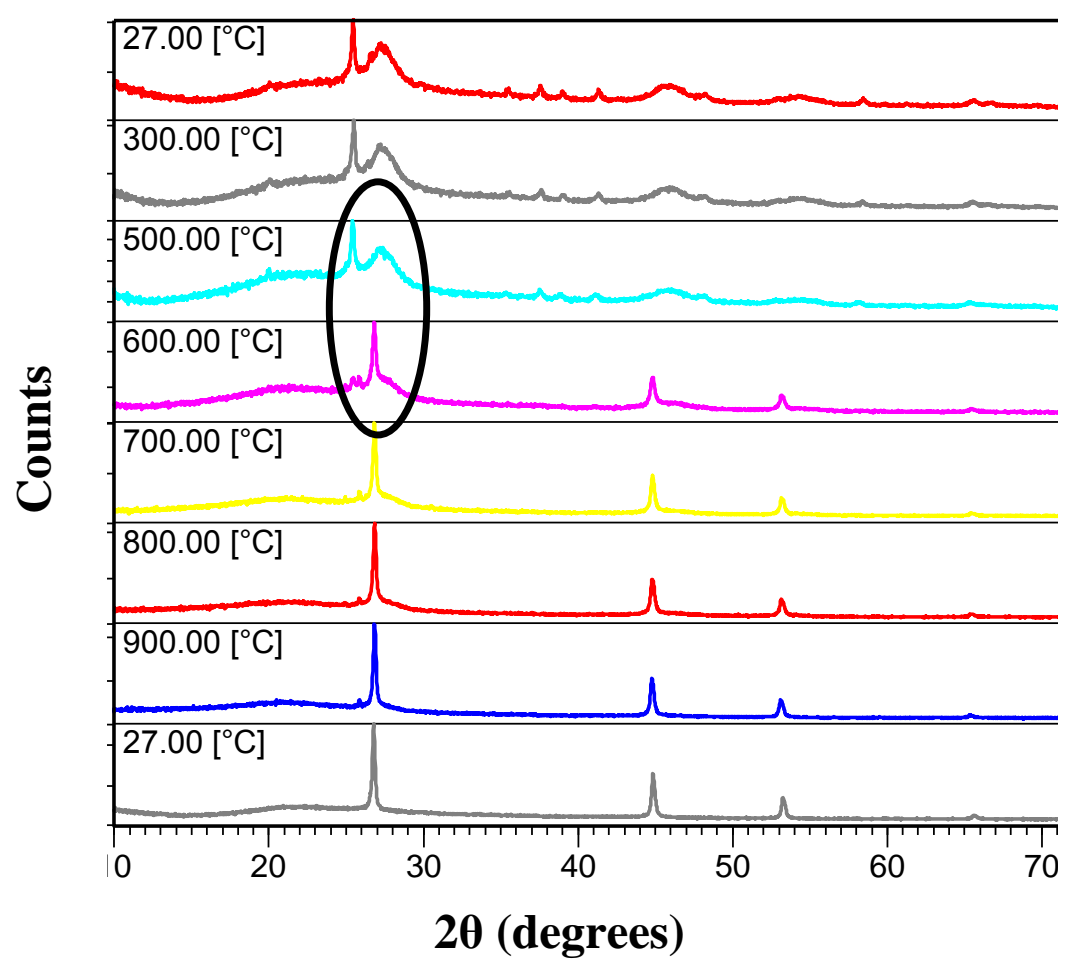

Figure 6.29. Si-Ge $5 \mathrm{~nm}$ nanocrystal phase stability by HT-XRD after only induction heating.

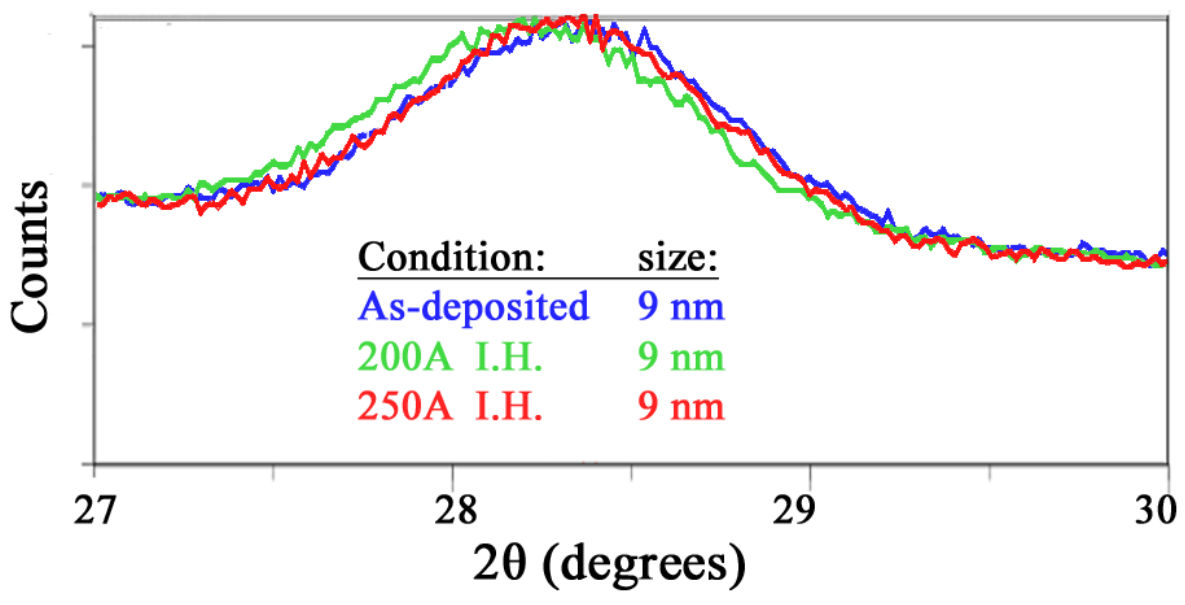

Figure 6.30. XRD of nanocluster Si-Ge (111) peak before and after 8 minutes of preheating.

To determine the laser sintering impact, further XRD characterization was also performed after laser hybrid sintering of $\mathrm{Si}_{0.8} \mathrm{Ge}_{0.2}$ nanoclusters on silicon <111> substrates. HighTemperature XRD (HT-XRD) was used to take scans from 20 to $100^{\circ}$ at room temperature, and then $700,800,900,1000,1100$, and $1200{ }^{\circ} \mathrm{C}$. Comparing the scans from this data, the laser sintered film is stable until between 1000 and $1100{ }^{\circ} \mathrm{C}$, at which point there is a slight increase in the $\mathrm{Si}$ (111) peak, although this peak is reduced again by $1200{ }^{\circ} \mathrm{C}$. Figure 6.31 shows three 
scans of this sample: the first at room temperature, the second at $1200{ }^{\circ} \mathrm{C}$, and the third after cooling back to room temperature. The primary Si-Ge (111) peak shifts due to thermal expansion from 27 to $1200{ }^{\circ} \mathrm{C}$ as expected, however the shape also changes. The lattice parameter of $\mathrm{Ge}$ is $\sim 4 \%$ larger than $\mathrm{Si}$, thus the more $\mathrm{Ge}$ concentration the further the Si-Ge peak will be shifted from the Si (111) peak to the Ge (111) peak. The shape change occurs because in the first scan, a large number of counts are from the bulk Si (111), whereas at $1200{ }^{\circ} \mathrm{C}$ those peak counts have dropped by a factor of 25 but the Si-Ge alloy signal has not been reduced as significantly. Upon returning to room temperature, the peak counts increased by a factor of 7 , but the Si (111) peak is still 3.5 times lower than originally. Therefore, with lower peak counts coming from the $\mathrm{Si}$ (111) peak at $28.5^{\circ}$, the Si-Ge contribution (from 27.8 to $28.4^{\circ}$ ) becomes relatively stronger which changes the shape. Furthermore, the signal for the Si-Ge shoulder comes from a different volume of the analyzed sample than does the $\mathrm{Si}$ (111). The $\mathrm{Si}-\mathrm{Ge}$ will be near the surface, whereas the deep penetration depth of the XRD beam (> $50 \mu \mathrm{m})$ also probes the bulk Si (111) wafer. The relatively low contribution of the Si (111) peak at $1200{ }^{\circ} \mathrm{C}$ may be explained by a decreased probing depth due to high thermal excitation of the lattice and thus a lower percentage of bulk contribution.

Effectively, the Si-Ge film and Si bulk areas act as two separate phases, and thus by specifying a unique reflection peak for each phase, further analysis on the crystallite size can be attempted. Using the Panalytical HighScore Plus software to analyze the shape of reflected signal, a best fit curve was generated for the 2 phases. This model assumes 2 volume regions:

- the sintered film where $\mathrm{Si}-\mathrm{Ge}$ of composition near Si0.8Ge0.2 is on the surface, - the bulk Si without Ge.

Figure 6.32 shows the curve fitting which generates a crystallite size for each of the 2 regions, as well as the signal strength from each region which at room temperature is approximated as the volume fraction of the total region probed by the beam. This estimation indicates that the Ge-rich region has a crystallite size as small as $25 \mathrm{~nm}$, but only amounts to $1.3 \%$ of the signal. Likewise, the bulk $\mathrm{Si}$ has a crystallite size in the micron range and accounts for $98.7 \%$ of the generated signal. There may be an interfacial diluted region which is not homogeneous, which would be a source of error. This model provides a first-order analysis of the structure of the laser sintered film, helping to understand the compositional variation vs. depth and indicating a very high density of crystal interfaces which improve phonon scattering. 


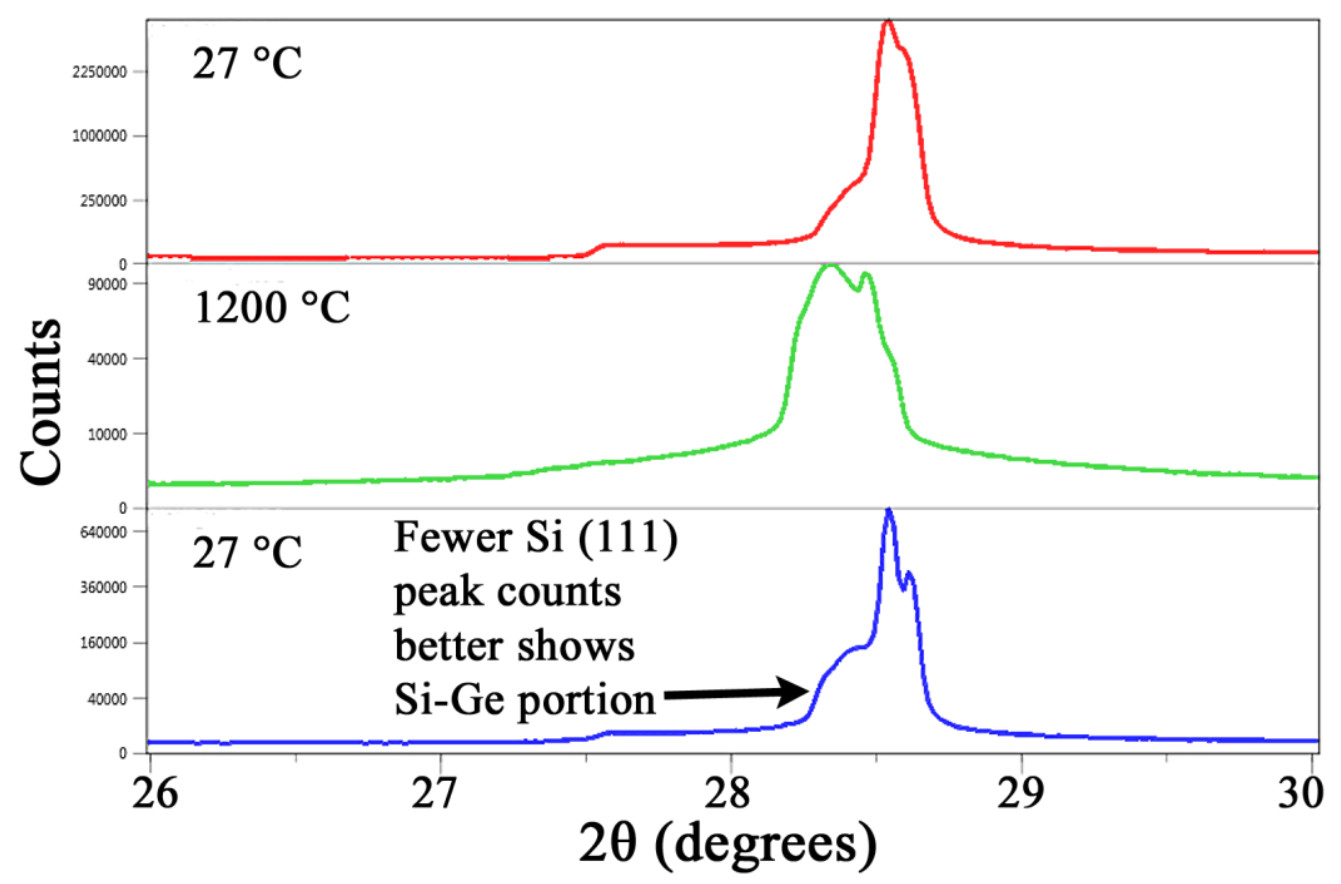

Figure 6.31. HT-XRD of Si-Ge (111) peak after laser sintering on Si substrate.

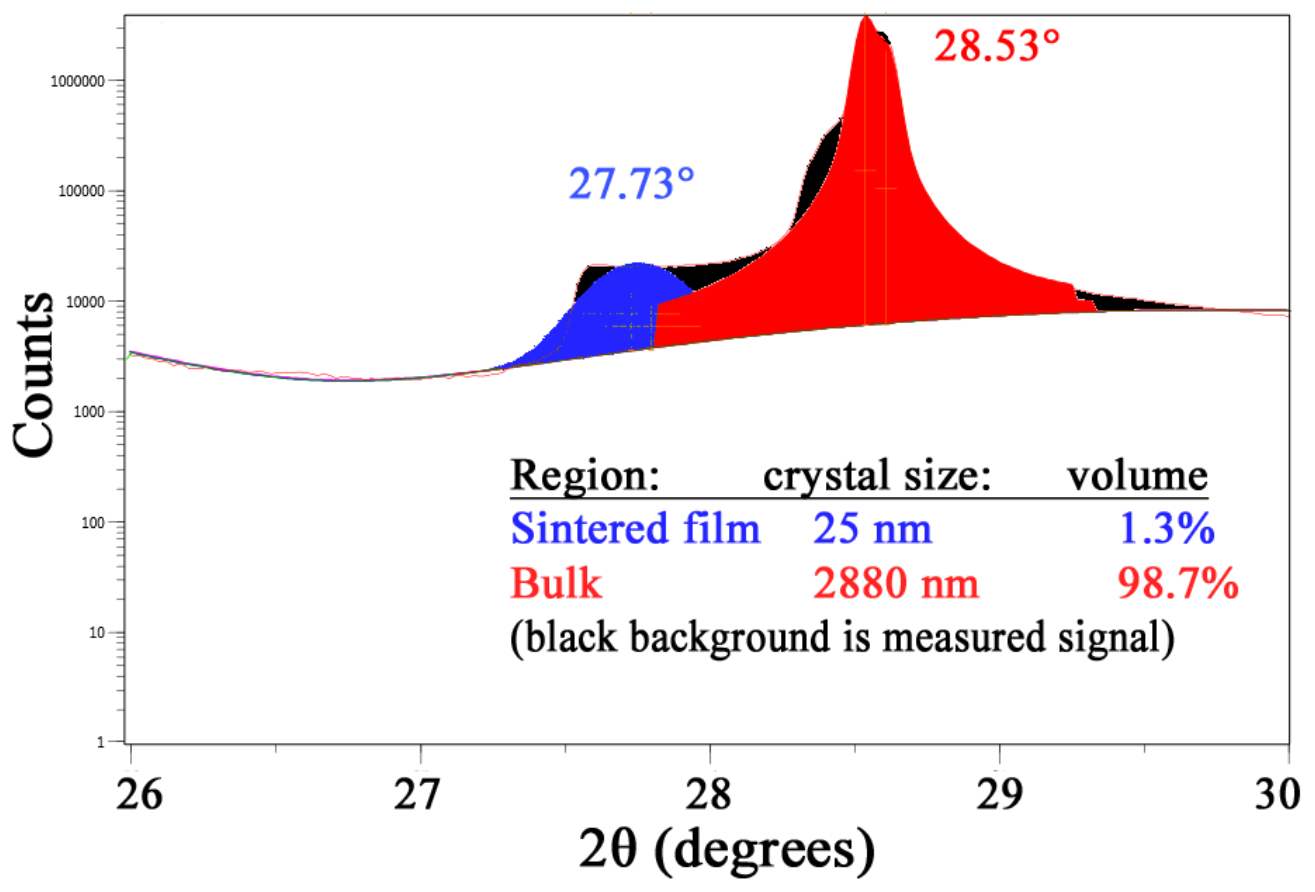

Figure 6.32. XRD of Si-Ge (111) peak after laser sintering on Si substrate. 
To understand the laser sintering impact on initial $\mathrm{Si}_{0.8} \mathrm{Ge}_{0.2}$ samples processed on fused silica substrates, one sample was characterized via HT-XRD. As before, the XRD scans were performed in an environment of $\mathrm{N}_{2}+4 \% \mathrm{H}_{2}$, at a scan rate of $\sim 1$ hour per temperature curve. The scan in Figure 6.33 showed no significant changes from room temperature through $900{ }^{\circ} \mathrm{C}$. The broad background peak at $22^{\circ}$ resulted from the fused silica substrate. All peaks related to silicon-germanium, but crystallite size exceeded the $\sim 100 \mathrm{~nm}$ detection limit of XRD. The CW laser with induction heating resulted in significant grain growth for this sample, thus the laser sintering conditions were adjusted to realize the lower thermal conductivity needed for improving ZT.

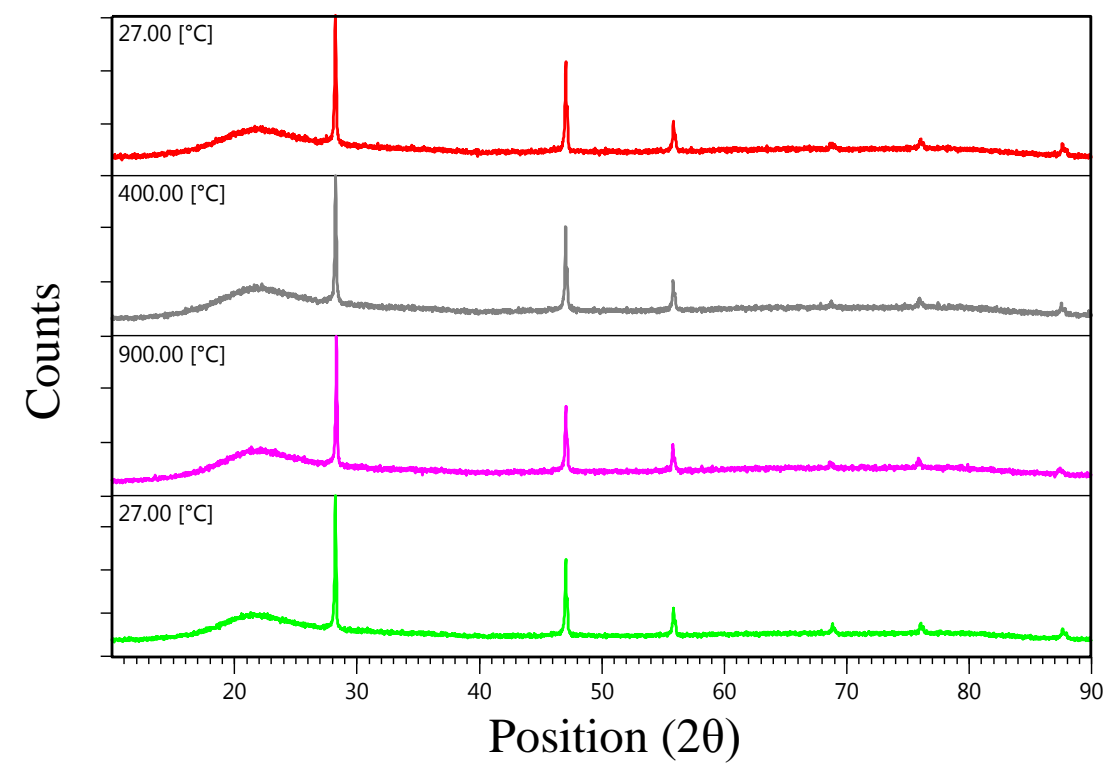

Figure 6.33. HT-XRD of Si-Ge (111) peak after laser sintering on silica substrate.

\subsubsection{Hall Mobility and Carrier Concentration}

Hall mobility measurements of samples sintered on silicon substrates were somewhat variable, but consistently gave a carrier concentration between 0.7 and $3.8 * 10^{19} / \mathrm{cm}^{3}$, mostly focused around $1.0 * 10^{19} / \mathrm{cm}^{3}$. As these values are lower than the ideal thermoelectric doping level for Si-Ge $\left(\sim 3 * 10^{20} / \mathrm{cm}^{3}\right)$, the carrier concentration data supports the conclusion that dopant unexpectedly diffused into the substrate during laser sintering. The mobilities were also analyzed at both liquid-nitrogen $(77 \mathrm{~K})$ and room $(295 \mathrm{~K})$ temperatures to determine if any temperature trends were observed, as this can help determine the scattering type of carriers. 
At higher doping levels, p-type Si-Ge materials with $<85$ at\% Ge can have lower mobility below room temperature than at room temperature. Measurements by Carlson indicate that for boron-doped Si the mobility at liquid-nitrogen temperature becomes less than the room temperature mobility at $\mathrm{p}$-type doping levels $\left(\mathrm{n}_{\mathrm{a}}\right)$ exceeding $\sim 2 * 10^{18} / \mathrm{cm}^{3}$ [146]. Carlson explains that high impurity concentrations can result in wave function overlap of the acceptor impurity band with the valence band, which may be prevented at very low temperature. The mobility peak tends to occur at lower temperatures for lower doping levels: below $100 \mathrm{~K}$ for $\mathrm{n}_{\mathrm{a}}<$ $\sim 1 * 10^{18} / \mathrm{cm}^{3}$, between 100 to $300 \mathrm{~K}$ for $\mathrm{n}_{\mathrm{a}} \sim 1-5 * 10^{18} / \mathrm{cm}^{3}$, and shifting to $>300 \mathrm{~K}$ at higher doping levels but with much less temperature variability. In summary, when the Hall mobility on p-type samples was lower or similar at $77 \mathrm{~K}$ versus $300 \mathrm{~K}$, then the doping level can be concluded to be $>1 * 10^{18} / \mathrm{cm}^{3}$.

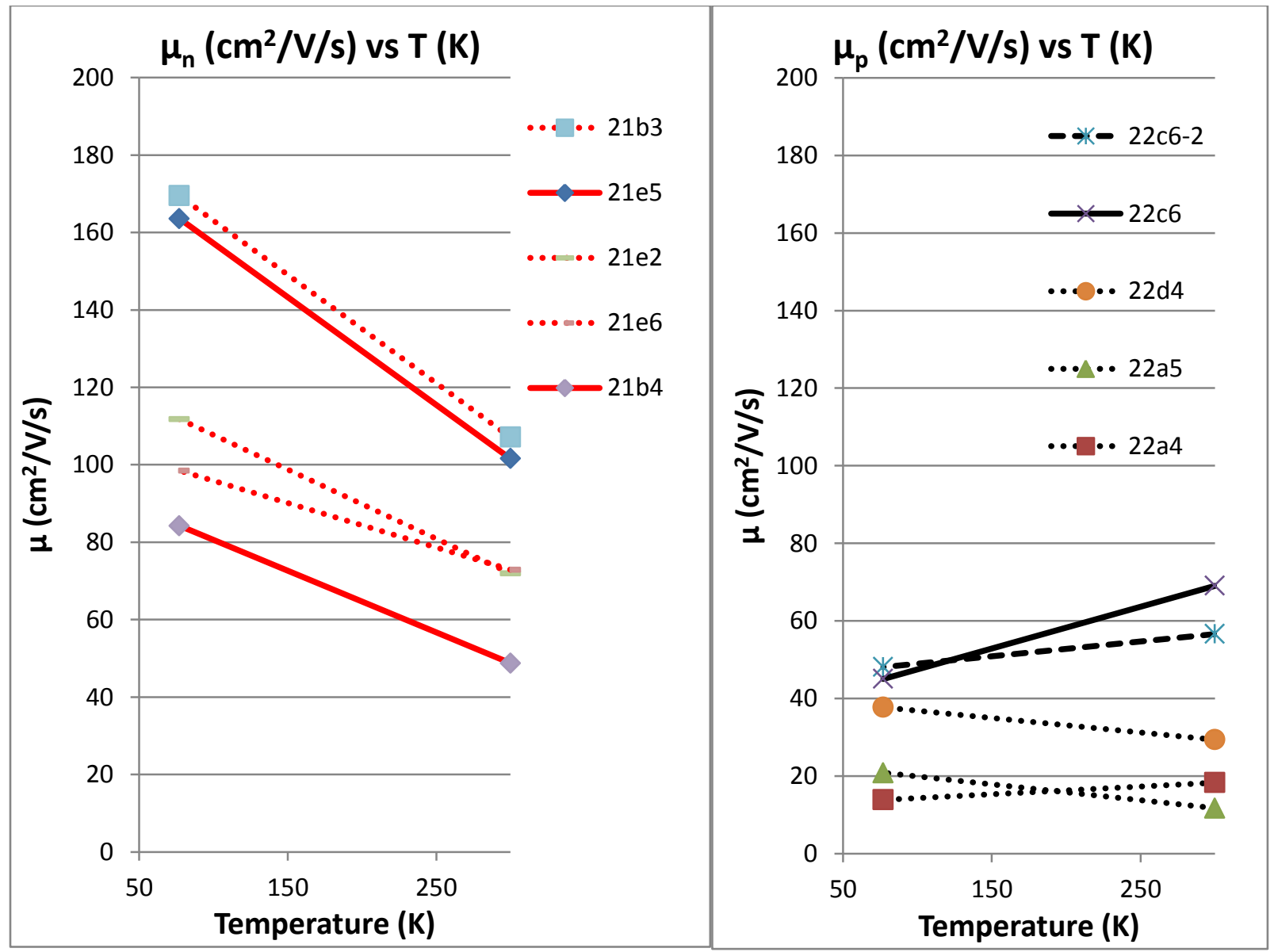

Figure 6.34. Hall mobility measurements on both n- and p-type samples on Si substrate. 
Figure 6.34 shows the measured mobilities of both n- and p- type samples. The dotted or dashed lines indicate that the sample underwent high-temperature characterization before mobility data was measured. The characterization temperatures were either 750,1000 , or 1500 $\mathrm{K}$, but it does not appear the thermal treatment significantly impacted the mobility values. The best values for both types of samples occurred when the relative laser processing was at slightly lower intensity, higher scan speed, and higher preheat temperature $\left(53 \mathrm{~W} / \mathrm{mm}^{2}, 12 \mathrm{~mm} / \mathrm{s}\right.$, and 250 amps of I.H). The measured mobility values for n-type tend to decrease from $77 \mathrm{~K}$ to $300 \mathrm{~K}$, whereas the p-type mobilities don't change consistently enough to draw any firm conclusions. The n-type mobility is generally higher, which is expected for Si-Ge. RTG bulk values for mobility are $\sim 50-55 \mathrm{~cm}^{2} / \mathrm{V} / \mathrm{s}$ for n-type and $\sim 30-35 \mathrm{~cm}^{2} / \mathrm{V} / \mathrm{s}$ for p-type [147]. Degenerately doped nanostructured Si-Ge materials typically have mobilities of $\sim 25-30 \mathrm{~cm}^{2} / \mathrm{V} / \mathrm{s}$ for n-type $[17,84]$ and $\sim 15-23 \mathrm{~cm}^{2} / \mathrm{V} / \mathrm{s}$ for p-type $[17,61,83]$, thus the higher mobility here also supports the determination that the carrier concentration is not at the ideal $2 * 10^{20} / \mathrm{cm}^{3}$.

\subsubsection{Electrical Resistivity at Room Temperature}

Room temperature resistance measurements were made on nearly all samples to provide quick feedback on the sintering results. The resistivity was then estimated based upon the sample geometry. Samples with poor percolation had high resistivities, often exceeding $1 \Omega \cdot \mathrm{cm}$, whereas samples with better morphology would be below $\sim 0.05 \Omega \cdot \mathrm{cm}$ with the best below 0.01 $\Omega \cdot \mathrm{cm}$. Depending upon the substrate used, the best achieved results at room temperature were:

- fused silica : $\quad 0.025 \Omega \cdot \mathrm{cm}$

- silicon: $\quad \sim 0.004 \Omega \cdot \mathrm{cm}$

- silicon carbide: $\sim 0.005 \Omega \cdot \mathrm{cm}$

The limitation with fused silica was bonding at the interface, and cracking due to thermal expansion mismatch. For silicon, dilution was a concern, but could be calculated by measuring resistance as a function of etching depth. For silicon carbide, the substrates tended to generate micro-cracks on the surface during laser scanning, thus the $\mathrm{SiC}$ may have had poor fracture toughness at high temperatures. These substrate cracks propagated through the entire thickness of the sintered film, preventing good macro-scale conduction in most cases. Solving the substrate issues would greatly facilitate obtaining resistivity values more competitive with the 
$\sim 0.001 \Omega \cdot \mathrm{cm}$ standard of bulk Si-Ge thermoelectric alloys. See section 4.1.4 for further discussion on substrate selection.

\subsubsection{Electrical Conductivity and Seebeck Coefficient to High Temperature}

High temperature thermoelectric performance was evaluated at HTML on two separate visits, once with laser sintered films on silicon substrates and once using fused silica substrates. Both times the measurements were conducted using the Ulvac ZEM-3 M8, which allows characterization up to $800{ }^{\circ} \mathrm{C}$. Owing to the bonding and associated intermixing with $\mathrm{Si}$ substrates, excellent film continuity was obtained, achieving electrical conductivity of $\sim 2 * 10^{4} /(\Omega \cdot \mathrm{m})$ at room temperature. Figure 6.35 graphs the electrical conductivity of the n-type samples vs. temperature, while Figure $\mathbf{6 . 3 6}$ graphs results from the p-type samples. The conductive depth was not known for all samples, thus in this case the absolute conductivity is extrapolated based on similar laser sintering conditions, and for these samples data points are not connected by a curve in the graph. The n-type laser sintered samples have conductivity about 5 times lower than state of the art bulk $n-\mathrm{Si}_{0.8} \mathrm{Ge}_{0.2}$ [78], and the p-type samples are about 10 times lower than the best bulk p-Si $\mathrm{Si}_{0.8} \mathrm{Ge}_{0.2}$ [77]. The lower conductivity results from the much lower than ideal doping concentration, which would need to be improved to be competitive. See section 6.3.3 for discussion about the relation of doping levels to electrical resistivity.

On the other hand, the lower doping levels facilitated very high Seebeck values. Figure 6.37 graphs the n-type Seebeck measurements vs. temperature while Figure 6.38 graphs results from the p-type samples. The values can be 1.5 to 3 times better than results from Wang et al. [78], and Joshi et al. [77], but only near room temperature. At higher temperatures, minority carrier generation erodes the thermopower $\left(S_{\text {total }}=S_{h}-\left|S_{e}\right|\right)$.

Of note is the increase in conductivity beginning at $\sim 400$ and $\sim 300{ }^{\circ} \mathrm{C}$ for $\mathrm{n}$ - and $\mathrm{p}$ - types, respectively. This type of increase can happen for 2 primary reasons in thermoelectrics: 1) excess dopant activates at higher temperatures and increases carrier concentration, or 2) doping level is sufficiently low that minority carrier generation begins to make an appreciable increase in the electrical conductivity. As this material is not near the solubility limit of $>1 * 10^{20} / \mathrm{cm}^{3}$, the data indicates substantial minority carrier generation begins at much lower temperatures than for degenerate Si-Ge. This effect should also begin decreasing the Seebeck coefficient at about the 
same temperature point, as confirmed in Figure 6.38, and may noticeably increase the thermal conductivity if the temperature is very high.

The measured Seebeck coefficients are all much higher than standard thermoelectric SiGe materials, and while this benefit could partly owe to carrier filtering from energy barriers created by sintering defects, the primary reason is again due to the lower than ideal carrier concentration. In fact, the p-type materials actually transition to become n-type materials by 400 $-600{ }^{\circ} \mathrm{C}$, following the trend that Geballe and Hull published for intrinsic Si [56] and Ge [57] at high temperatures.

Combining the Seebeck coefficient and electrical conductivity measurements, Figure 6.39 graphs the power factor of laser sintered samples on Si substrates. The laser sintered p-type material certainly does not compare well to the best bulk materials [77], however the n-type $\mathrm{Si}$ Ge compares favorably at all temperatures and even obtains the best performance below 350 ${ }^{\circ} \mathrm{C}$ [78]. This excellent low temperature performance shows that the material can be optimized for a specific temperature range by choosing the appropriate doping level.

These results demonstrate that successful doping can be achieved with laser sintering. Furthermore, by choosing an inert substrate, dilution can be avoided as demonstrated in Figure 6.26. Initial conductivity measurements when sintered on silicon carbide substrates already show performance similar to Si substrate, reaching $\sim 0.2 * 10^{5} /(\Omega \cdot \mathrm{m})$ [resistivity $\left.\approx 5 \mathrm{~m} \Omega \cdot \mathrm{cm}\right]$. Plus, the lack of dilution with the $\mathrm{SiC}$ substrate should ensure much higher dopant concentration in the sintered film, and thus low minority carrier density would result in achieving the best performance at higher temperatures $\left(\sim 600-800^{\circ} \mathrm{C}\right)$. 


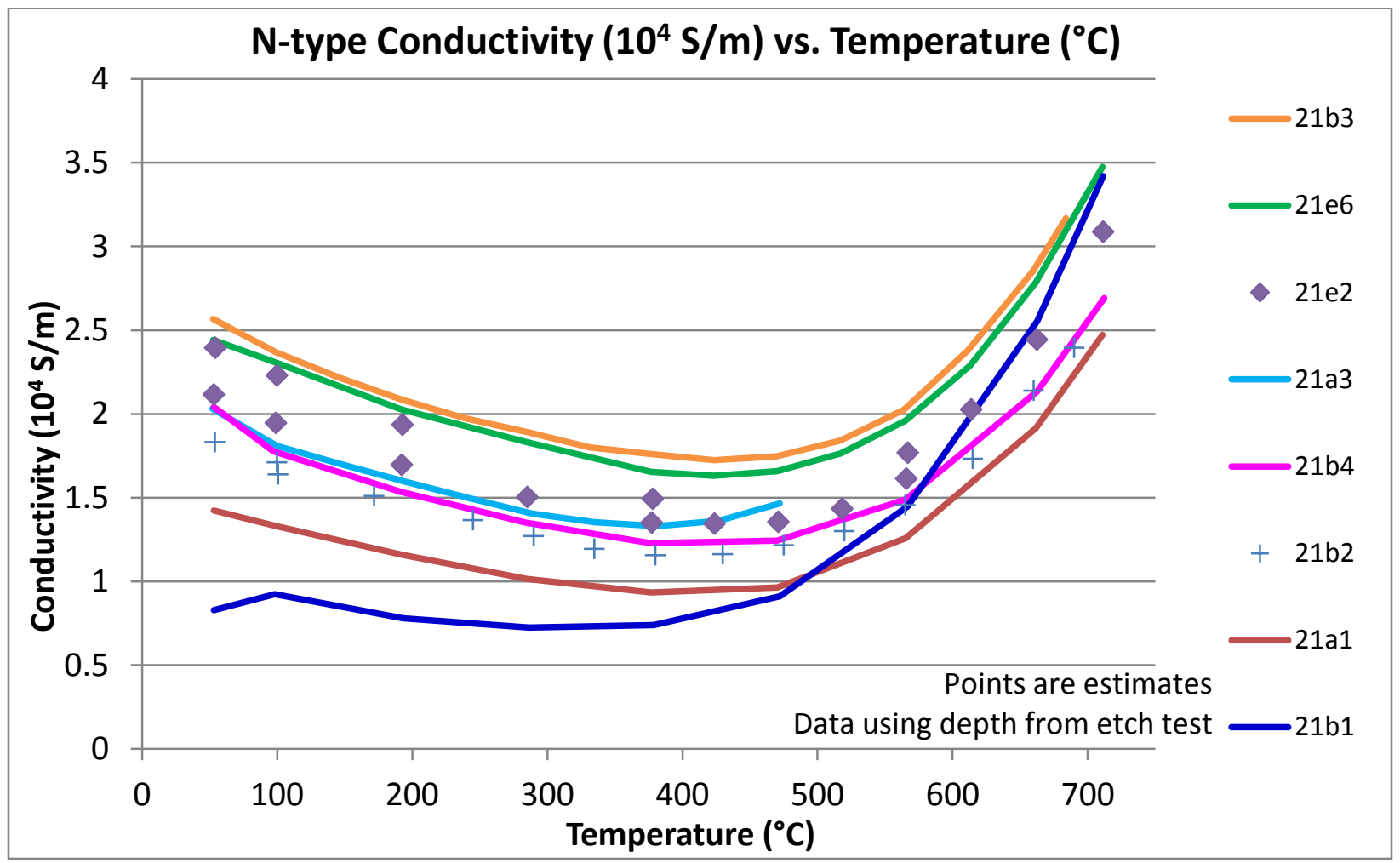

Figure 6.35. Electrical conductivity vs. temperature for n-Si-Ge on $\mathrm{Si}$.

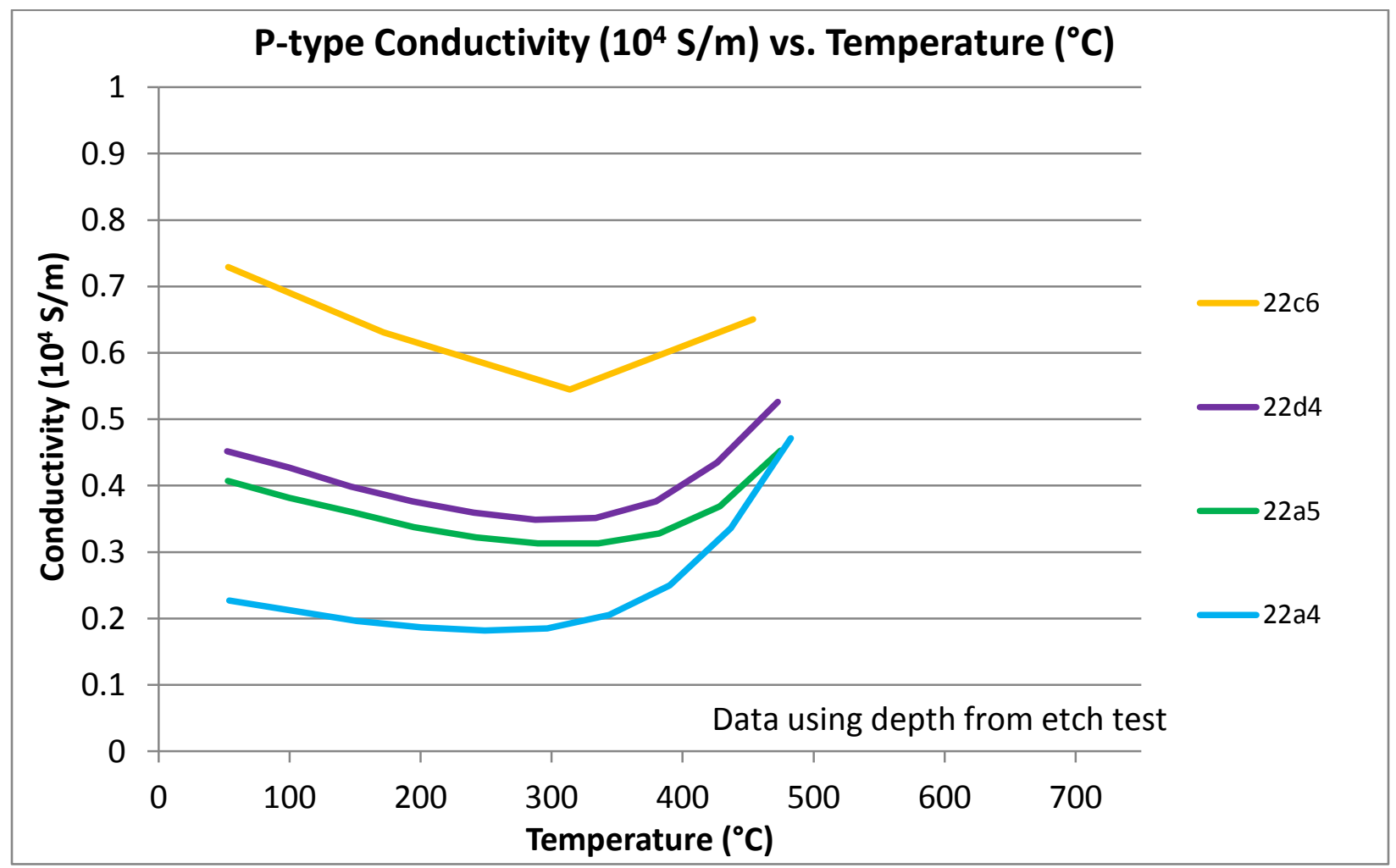

Figure 6.36. Electrical conductivity vs. temperature for p-Si-Ge on $\mathrm{Si}$. 


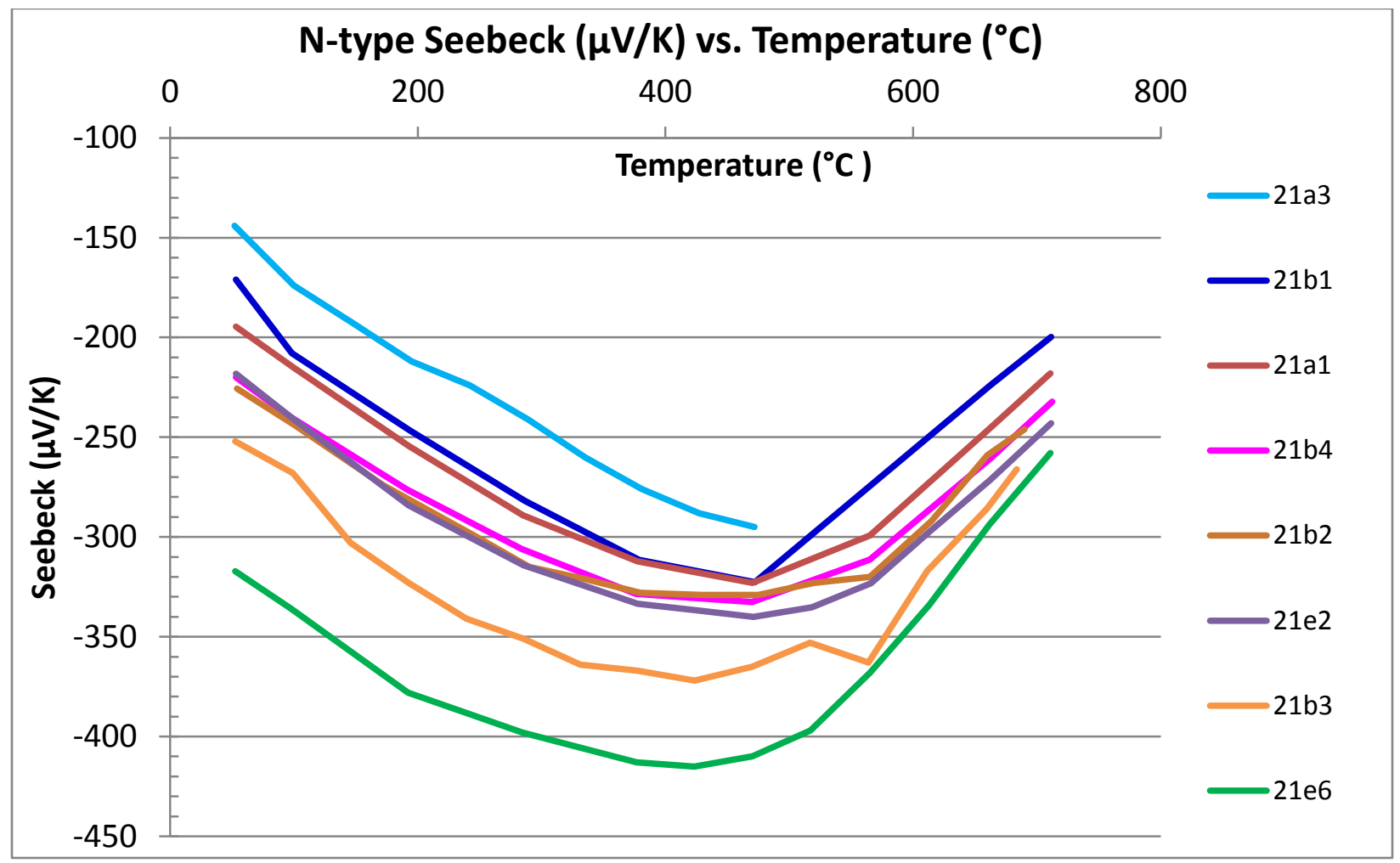

Figure 6.37. Seebeck vs. temperature for n-Si-Ge on Si.

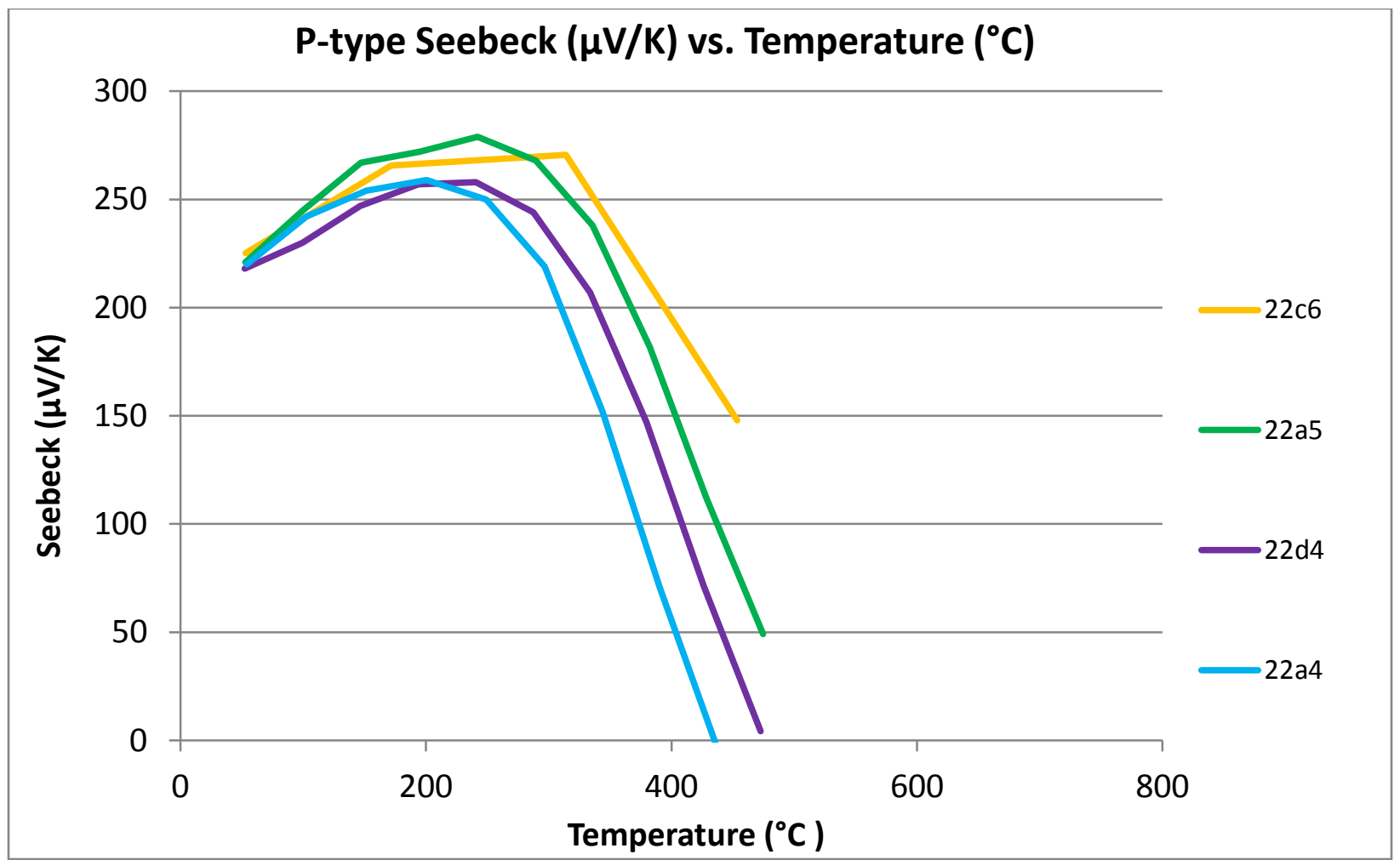

Figure 6.38. Seebeck vs. temperature for p-Si-Ge on Si. 


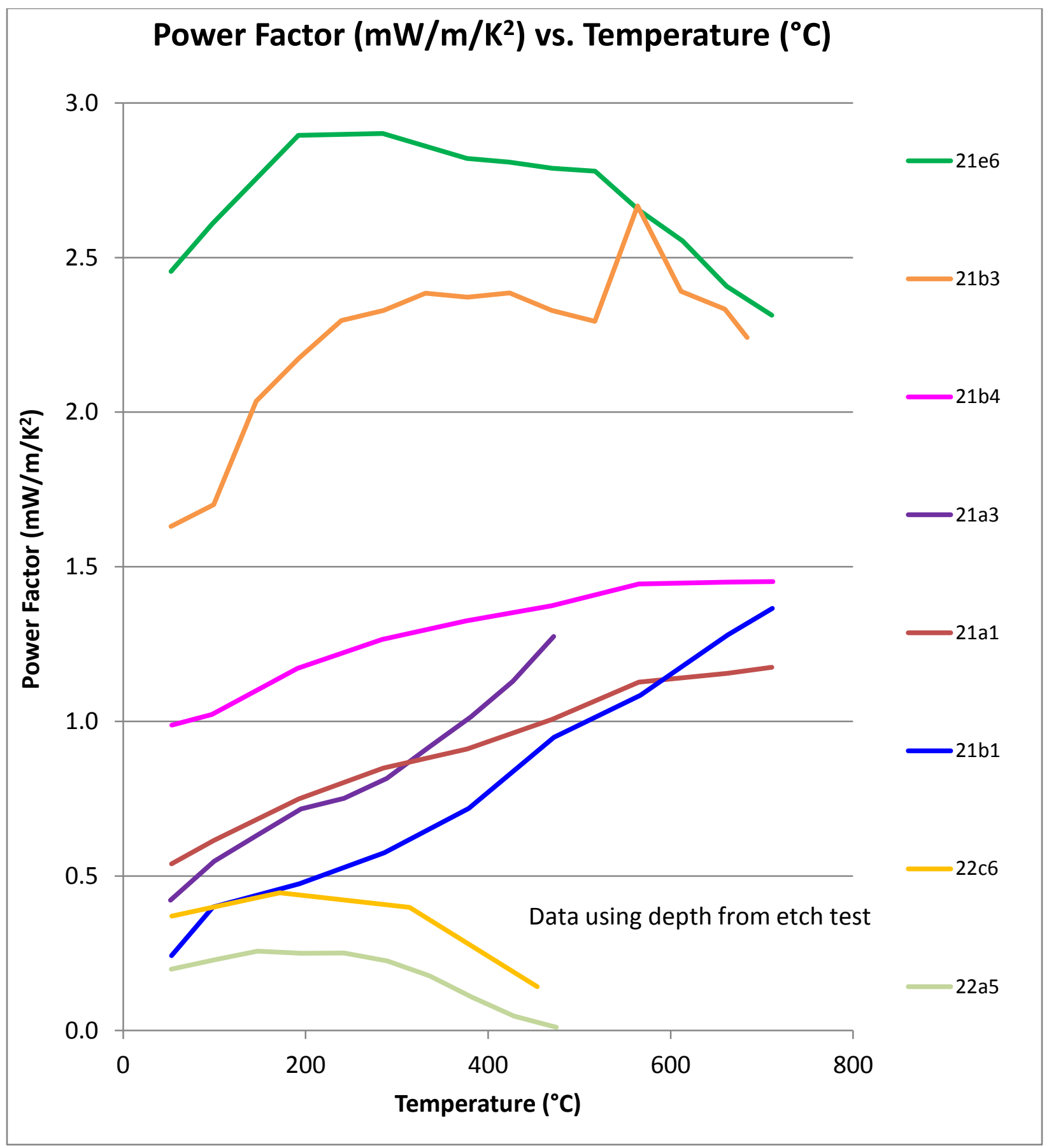

Figure 6.39. Power Factor vs. Temperature for laser sintered Si-Ge on Si. 
In addition to the high temperature measurements using silicon substrates, initial work was performed using fused silica substrates. These results also had high Seebeck coefficients, but the conductivity was quite low due to restricted percolation through the film. The best measured conductivity was $\sim 0.2 * 10^{4} /(\Omega \cdot \mathrm{m})$. The electrical properties of one p-type and one $\mathrm{n}$ type device were individually tested in the same Ulvac ZEM-3; unfortunately, this ZEM-3 had been upgraded from a ZEM-2, and therefore had hardware unable to measure resistance larger than $\sim 10 \mathrm{k} \Omega$, which was restrictive for these thin films. The phosphorus-doped sample was not measurable in the ZEM-3, due to total resistance exceeding the $10 \mathrm{k} \Omega$ limit. The resistance of the boron-doped sample was low enough to take measurements with the ZEM-3, however the gold contacts formed a eutectic with the silicon at $363{ }^{\circ} \mathrm{C}$ at which point the electrical probes lost connectivity with the sample. This resistivity and thermopower data are graphed to $360{ }^{\circ} \mathrm{C}$ in Figure 6.40. Also noteworthy is that the resistivity measurements were reduced by up to a factor of 5 due solely to ambient room light if the probing current level was not significantly higher than a few microamps. At these low current levels, the photogenerated current overwhelmed the multimeter signal.

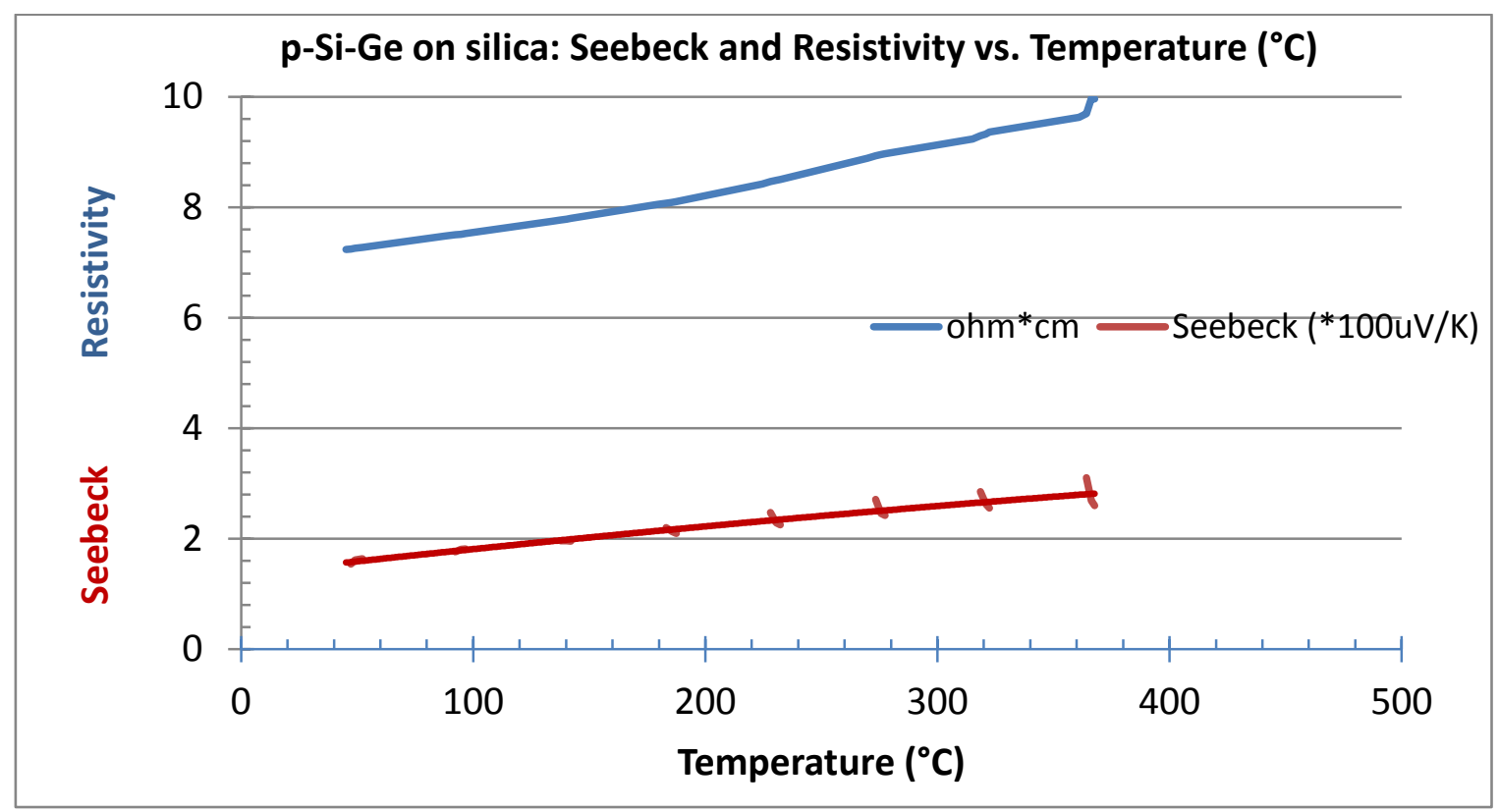

Figure 6.40. p-Si-Ge thin-film thermopower and resistivity after laser sintering. 


\subsubsection{Thermal Conductivity to High Temperature}

These results for thermal conductivity were obtained on laser sintered Si-Ge samples on fused silica or silicon substrates using the Time-Domain ThermoReflectance (TDTR) technique, in collaboration with Prof. Patrick Hopkins and Ramez Cheaito.

Samples were polished to reduce the taller peaks from $\sim 6 \mu \mathrm{m}$ to $\sim 2 \mu \mathrm{m}$, although the base layer was still only $\sim 500 \mathrm{~nm}$ thick. The plateaus formed during polishing were nearly $100 \%$ dense, and provided a specular reflection to enable thermal characterization, as exemplified in Figure 6.41. In this optical image, the dark regions are the polished plateaus which provide a stronger signal for TDTR measurements. Electrical resistivity of samples was measured by 4 point probe to determine the electronic contribution to thermal conductivity.

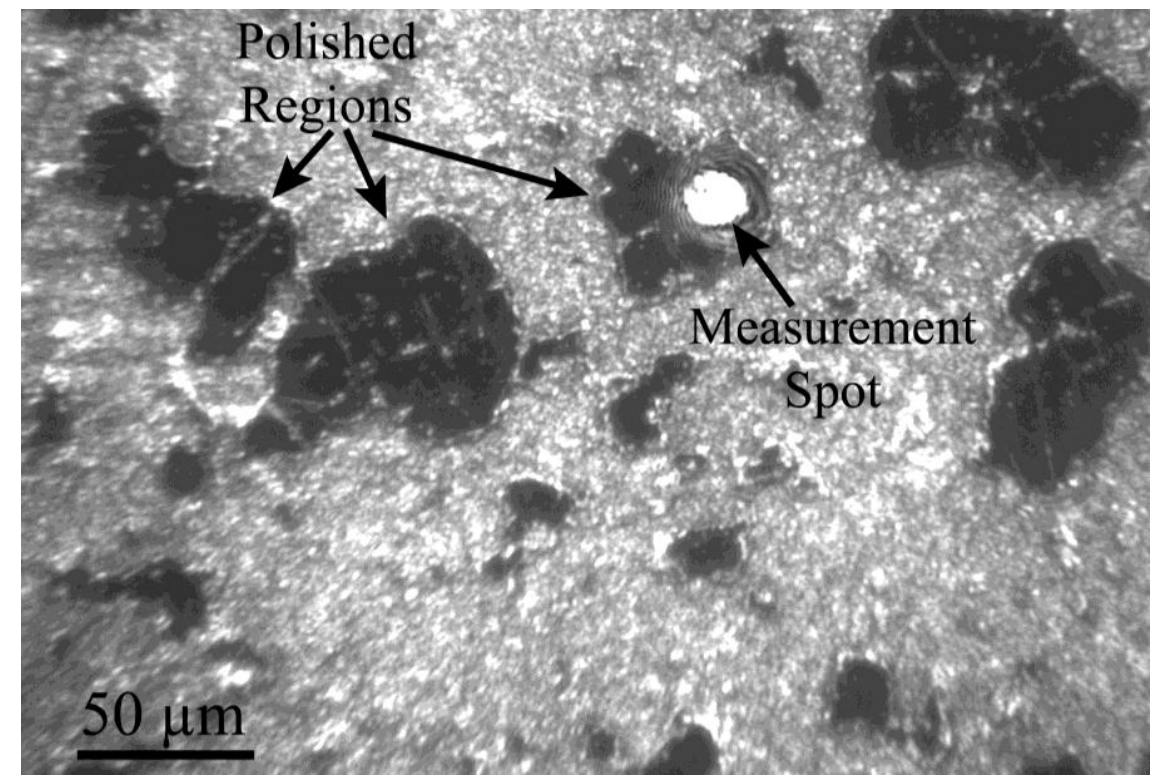

Figure 6.41. TDTR rough sample surface example, with probe spot size.

Improved laser sintering parameters facilitated the achievement of extremely low thermal conductivity values for $\mathrm{Si}-\mathrm{Ge}$, as shown in Figure 6.42. The bulk $\mathrm{Si}_{0.8} \mathrm{Ge}_{0.2}$-alloy curve is from Lee et al. [148], the MIT/BC curve is from Wang et al. [78], and the $\alpha-\mathrm{Si}_{0.8} \mathrm{Ge}_{0.2}$ curve is a linear interpolation ( $80 \% \alpha-\mathrm{Si}+20 \% \alpha-\mathrm{Ge})$ between $\alpha$-Si from Cahill's Debye model [149] and $\alpha-\mathrm{Ge}$ curve from Lee et al. [148]. The $\alpha-\mathrm{Si}_{0.8} \mathrm{Ge}_{0.2}$ curve is used to estimate the ideal phonon glass condition considered to be the minimum achievable condition without 1D or 2D nanostructures. The room-temperature value is significantly better than demonstrated using the DC hot pressing 
method by Wang et al. [78], and validates the hypothesis that laser sintering can enable lower thermal conductivity for Si-Ge. The thermal conductivity value of $1.36 \mathrm{~W} / \mathrm{m} / \mathrm{K}$ at room temperature is lower than any reported value for the Si-Ge system of which the author is aware that doesn't feature 1D or 2D nanostructures, despite still comparing well to nanowires and superlattices [71].

For the laser sintered results, $\kappa_{p h}$ is approximated with a logarithmic curve giving $\kappa=$ $0.428 * \ln (T)-1.07$, where the units of $\kappa$ are (watts per meter per Kelvin) and $\mathrm{T}$ is in Kelvin, and ignoring the infrequently noticed porosity which likely increased scatter of the data. As typical for sintered $\mathrm{Si}-\mathrm{Ge}$ there is some oxide concentration built in to the layer, which is often ignored so long as the electrical performance is sufficient. Any oxide concentration is not expected to play a large role in the thermal conductivity value given the nano-size grains already scatter phonons effectively and the phonon mean free path is much longer than the 1-2 nm thickness of the typical oxide interfaces. It's also important to note that EDS shows the UMN nanoclusters may have a low carbon impurity, which could also play a small role in enhancing phonon scattering due to the additional variance in atomic number and elastic modulus. Further study is needed to test the impact of porosity or excessive oxide concentration on $\kappa$, although SEM imaging in Figure 6.25 and initial crystallite size analysis in Figure 6.32 support that the laser sintered Si-Ge has a very high density of interfaces which would thus be expected to provide significantly reduced thermal conductivity as was measured. High-resolution TEM images also confirm a large number of crystallite orientations within each grain. 


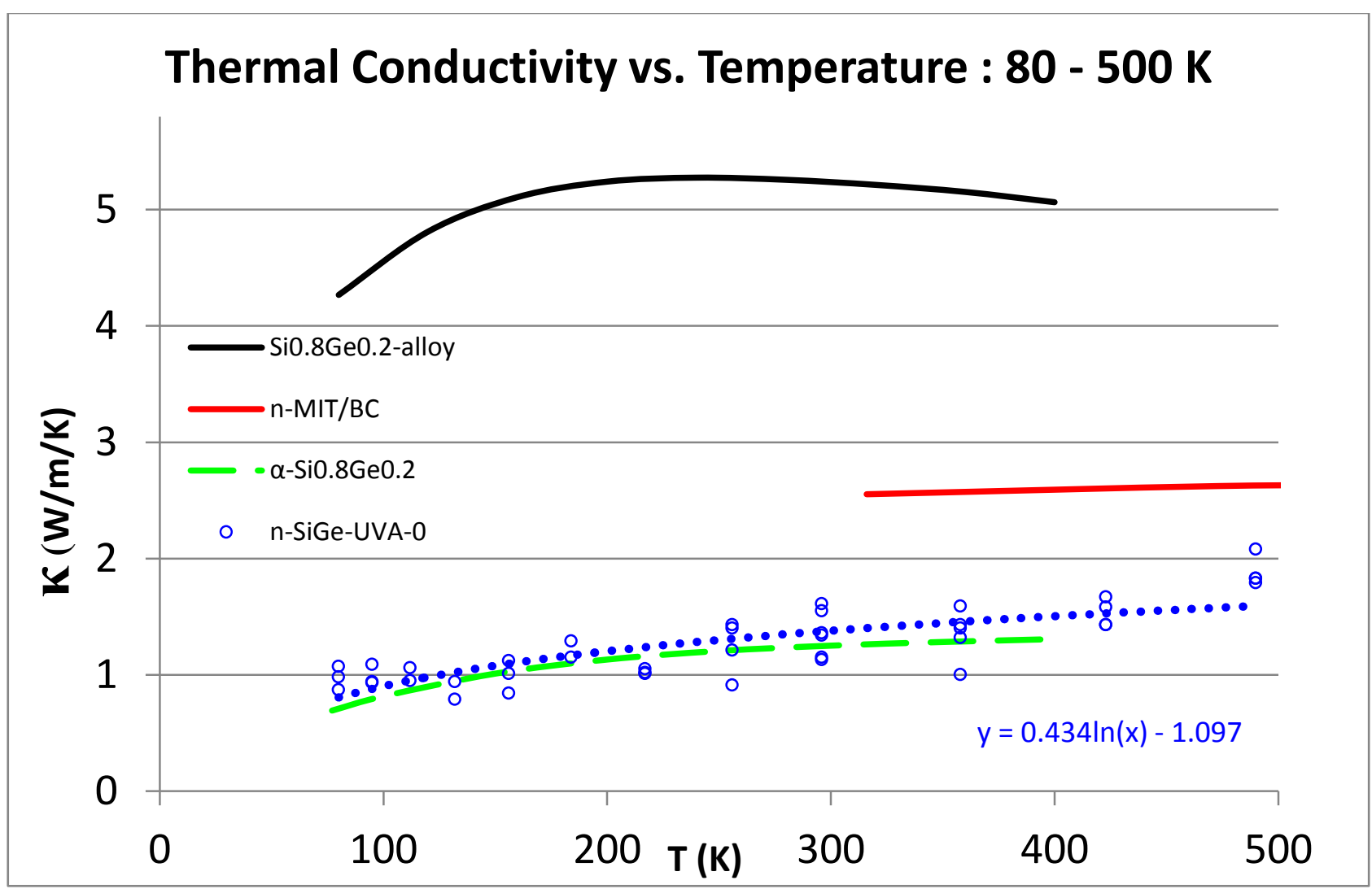

Figure 6.42. Thermal conductivity low-temperature results on fused silica substrate.

The laser sintered results for thermal conductivity are compared with Si-Ge literature values in Figure 6.43, including superlattices and nanowires, except that the UVA, MIT/BC and RTG curves show only the calculated phonon contribution $\left(\kappa_{p h}\right)$ for a more consistent comparison. The $\kappa_{e}$ contribution is found using Eq. 2-S, where the Lorenz number is calculated using Eq. 2-K in combination with the scattering parameter calculated from Table C. The bulk $\mathrm{Si}_{0.8} \mathrm{Ge}_{0.2}$-alloy, $\mathrm{Si}_{0.85} \mathrm{Ge}_{0.15}-1 \mu \mathrm{m} \_$film, $\mathrm{Si}_{0.69} \mathrm{Ge}_{0.31}$-Superlattice, and $\alpha$-Ge curves are from Lee et al. [148]. The $\mathrm{Si}_{0.9} \mathrm{Ge}_{0.1}-\mathrm{NW}-\mathrm{Superlattice}$ curve is from $\mathrm{Li}$ et al. [150] and the $\mathrm{Si}_{0.5} \mathrm{Ge}_{0.5^{-}}$ Superlattice curve is from Borca-Tasciuc et al.[151]. The n-MIT/BC-ph and n-RTG-ph curves are best estimates of $\kappa_{p h}$ from Wang et al. [78]. The $\alpha$-Si curve is from Cahill's Debye model [149], which best matches experimental values. As shown in the graph, the UVA laser sintered sample has lower thermal conductivity than all samples except amorphous germanium and a $\mathrm{Si}_{0.5} \mathrm{Ge}_{0.5} 4 \mathrm{~nm}$ superlattice doped to $\sim 2 * 10^{18} \mathrm{~cm}^{-3}$. 


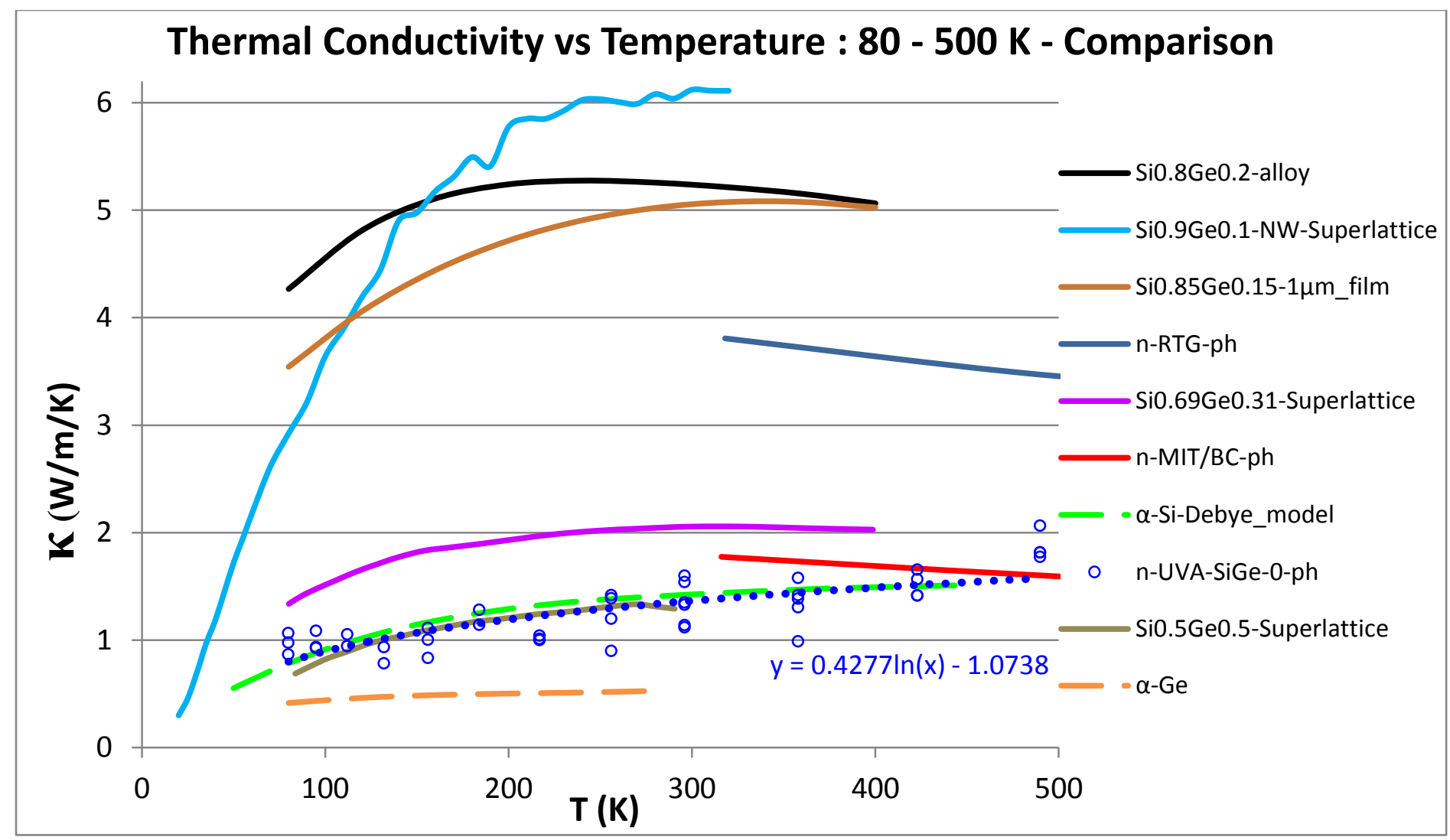

Figure 6.43. Thermal conductivity low-temperature comparison to literature.

The remaining thermal characterization was performed on samples sintered on intrinsic $\mathrm{Si}<111>$ substrates. Table $\mathbf{H}$ provides a list of the room temperature TDTR measurements along with the major variables studied for the preheating and laser sintering conditions for these samples. The thermal conductivity measurements on Si substrates remained low despite dopant dilution into the substrate, which supports the XRD model of a two phase system with $\mathrm{Si}_{0.8} \mathrm{Ge}_{0.2}$ remaining undiluted on the surface, as shown in Figure 6.32. Considering the TDTR probe only analyzed 300-400 nm of Si-Ge depth, it remains mostly unaffected by the high thermal conductivity of the bulk Si layer underneath the laser sintered nanocluster film.

Sample $A$ was processed at a laser power density of $73 \mathrm{~W} / \mathrm{mm}^{2}$ and sample $B$ at $79 \mathrm{~W} /$ $\mathrm{mm}^{2}$, with the remaining major parameters as $12 \mathrm{~mm} / \mathrm{s}, 0.4 \mathrm{~mm}$ step, and with preheating at 125 amps. Sample $A$ had a significantly lower measured $\kappa=2.16+/-0.15$, whereas for sample $B \kappa=$ $5.2+/-0.3$. As the concentration of Ge decreases, $\kappa$ is expected to increase significantly. Thus, this change is likely due to more than just additional grain growth from laser heat, but rather from composition changes as a function of depth. Another significant aspect to the thermal 
measurements was the variation for areas that were less polished, i.e., rougher surfaces. These areas almost always had lower measured thermal conductivity, also likely due to less Ge dilution into the substrate as they were not at the center of the Gaussian laser sintering beam but rather were nearer the edge. This occurs because the TDTR probe beam is $\sim 25$ micron in diameter, whereas the laser sintering beam was $\sim 880 \mu \mathrm{m}$, therefore the TDTR measurements can individually probe the different locations within a single laser linescan. Measurement uncertainly is expected to be $\sim 10 \%$. As the samples first had to be polished before the $\sim 80 \mathrm{~nm}$ aluminum transducer layer was deposited, some of the sintered nanocluster layer was removed, which could result in higher measured $\kappa$ values. The only value for the 'rough' region which exceeded $2 \mathrm{~W} / \mathrm{m} / \mathrm{K}$ was at the highest laser intensity, where visible melting of the substrate was noted after the experiment was run, and the laser was never used at this high of intensity again for Si substrates. The heat capacity of Si-Ge was assumed to be $1.66 \mathrm{~J} / \mathrm{cm}^{3} / \mathrm{K}$ taken from Table B for these measurements; the value used has less than $1 \%$ error for the composition range of $\mathrm{Si}_{1} \mathrm{Ge}_{0}$ to $\mathrm{Si}_{0.8} \mathrm{Ge}_{0.2}$, assuming no porosity. It should be noted, however, that occasional porosity has been observed, so total uncertainty may increase above $10 \%$.

Table H - Thermal conductivity measurements on Si substrates at $300 \mathrm{~K}$.

\begin{tabular}{|l|l|c|c|c|c|c|}
\hline & TDTR & Intensity & I.H. & speed & step & K (W/m/K) \\
\hline ID & Sample & W/mm & amps & $\mathrm{mm} / \mathrm{s}$ & $\mathrm{mm}$ & smooth \\
\hline$A$ & 22a1-c & 73 & 125 & 12 & 0.4 & $\mathbf{2 . 3 6}$ \\
\hline$B$ & 22a1-d & 79 & 125 & 12 & 0.4 & $\mathbf{6 . 0 7}$ \\
\hline$C$ & 22c5-c & 62 & 200 & 12 & 0.4 & $\mathbf{3 . 6 4}$ \\
\hline$D$ & 22c5-d & 65 & 200 & 12 & 0.4 & $\mathbf{1 . 1 6}$ \\
\hline$E$ & 22c5-e & 68 & 200 & 12 & 0.4 & $\mathbf{5 . 0 4}$ \\
\hline$F$ & 22d1-d & 65 & 160 & 12 & 0.4 & $\mathbf{6 . 8 5}$ \\
\hline$G$ & 22d3-c & 53 & 250 & 12 & 0.4 & $\mathbf{0 . 8 6}$ \\
\hline$H$ & 22d3-d & 56 & 250 & 12 & 0.4 & $\mathbf{0 . 8 8}$ \\
\hline
\end{tabular}

High temperature measurements were taken on 3 samples, 2 n-type from the same processing conditions and $1 \mathrm{p}$-type. The results are somewhat challenging to interpret, as the $\mathrm{n}$ and p- samples unexpectedly have different temperature dependencies. The results are graphed for p-type in Figure 6.44 and for n-type in Figure 6.45, where the low-temperature sample is also included for comparison. The dashed lines indicate higher measurement uncertainty. 
For the p-type material, using a $1 / \mathrm{T}$ plot reveals that the thermal conductivity has a $\mathrm{T}^{-0.9}$ dependence for this sample. While $\kappa$ is expected to decrease with temperature, literature does not support this curve shape, including the referenced MIT/BC and NASA RTG samples [77].

For the n-type material, the thermal conductivity of the laser sintered samples compares quite well with literature values $[77,78]$, perhaps the best recorded below $150{ }^{\circ} \mathrm{C}$. Based on the thermoelectric measurements where the Seebeck value peaked at 500 / $700 \mathrm{~K}$ for p- / n- type, respectively, the ambipolar contribution should begin increasing $\kappa$ at these temperatures, which helps explain the increasing value measured for the n-type sample. It is possible that up to 1.5 $\mathrm{W} / \mathrm{m} / \mathrm{K}$ was due to $\kappa_{b p}$ for the $\mathrm{n}$-type samples at $800{ }^{\circ} \mathrm{C}$, although a more conservative model [Eq. 2-T] was used for Figure 6.45 which gave $\kappa_{b p}=1.0 \mathrm{~W} / \mathrm{m} / \mathrm{K}$ at $800{ }^{\circ} \mathrm{C}$. The significant ambipolar contribution is not surprising for this Si-Ge material given that the low $\sim 1 * 10^{19} / \mathrm{cm}^{3}$ doping level allows high minority carrier generation at high temperatures. In this case the majority and minority carriers both transfer heat from the hot to cold regions but the net charge transfer is zero (since holes and electrons have opposite charges), which is why $\kappa_{b p}$ is detrimental to good high temperature thermoelectric performance. An inverse temperature plot can assist with ambipolar analysis [59] but for this method to work the thermal conductivity must change from decreasing to increasing as temperature increases.

The electronic contribution $\kappa_{e}$ for n-UVA-SiGe-1 and n-UVA-SiGe- 2 is projected to be $1.0 \mathrm{~W} / \mathrm{m} / \mathrm{K}$ at $800{ }^{\circ} \mathrm{C}$, but as electrical measurements stopped at $700{ }^{\circ} \mathrm{C}$ the value is just a linear estimate of the electrical conductivity data. By subtracting the ambipolar and electronic portions, the estimated lattice contribution has been included in the graph to assist comparison with the referenced literature [78], and to help understand why the total thermal conductivity increased with temperature. While $\kappa_{p h}$ still increases somewhat from 200 to almost $600{ }^{\circ} \mathrm{C}$, the slope is not as strong as total $\kappa$. The dip at 50 to $100{ }^{\circ} \mathrm{C}$ is not directly explainable considering only the laser sintered portion; perhaps the Pt transducer or $\mathrm{Si}_{3} \mathrm{~N}_{4}$ barrier layer changed slightly, although the measured values were repeatable even after measurements at $500{ }^{\circ} \mathrm{C}$. One proposed explanation is that the films were able to incorporate moisture at the lower temperatures which prevented proper thermal conductance from the $\mathrm{Pt}$ to the $\mathrm{Si}-\mathrm{Ge}$ layer. Above $600{ }^{\circ} \mathrm{C}$ the stability of the $\mathrm{Si}_{3} \mathrm{~N}_{4}$ barrier layer between the $\mathrm{SiGe}$ and $\mathrm{Pt}$ is unknown, so these values are not used for evaluation, instead only included for completeness. 


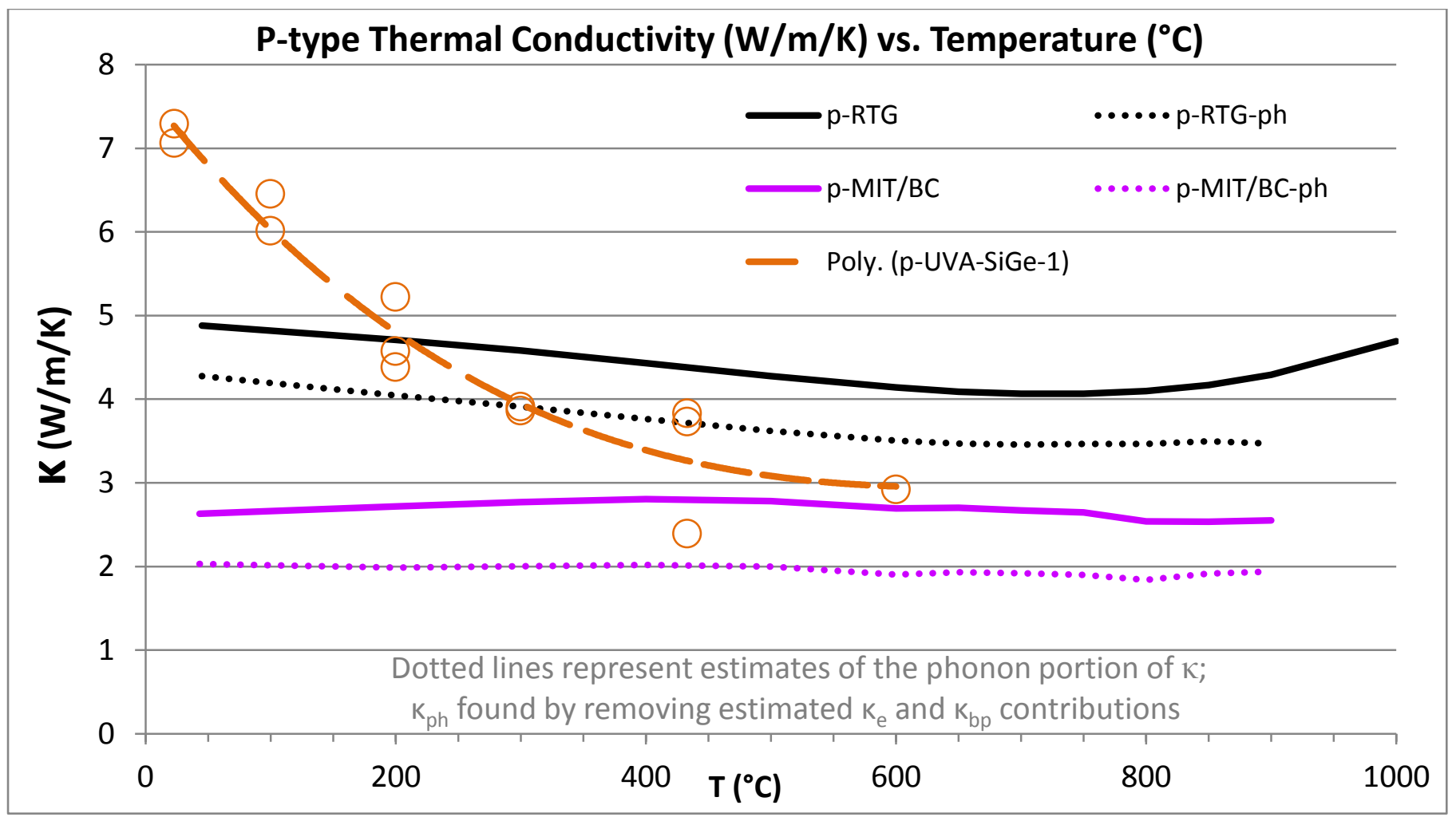

Figure 6.44. Laser sintered p-type TDTR measurements vs. temperature on Si substrate.

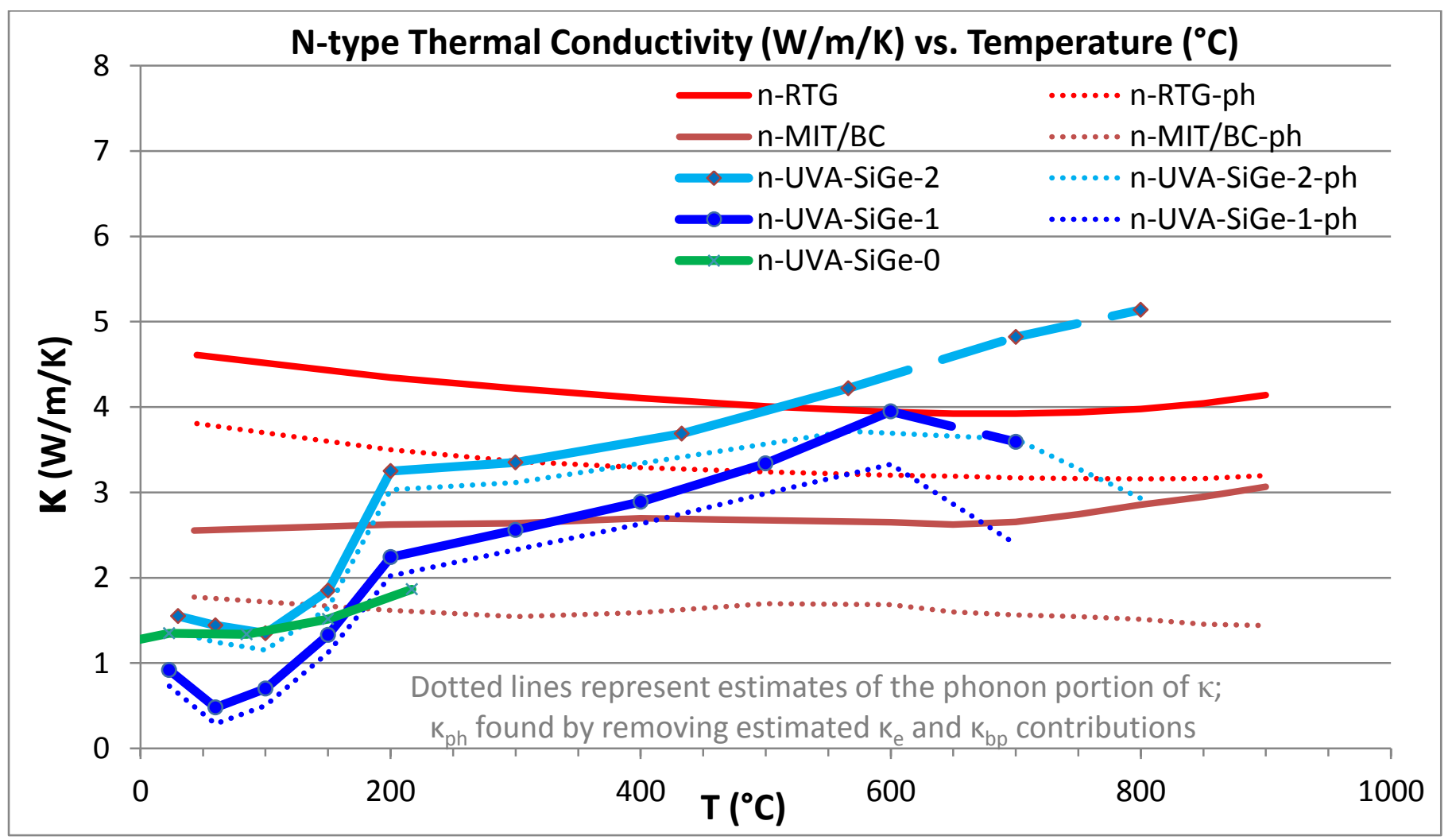

Figure 6.45. Laser sintered n-type TDTR measurements vs. temperature on Si substrate. 


\subsubsection{Summary}

Laser sintering of doped silicon germanium nanoclusters has been shown as a successful way to achieve a nearly full-density continuous film with good electrical conductivity and excellent thermal resistivity, especially at temperatures nearer to room temperature. Laser sintering a film to achieve a low final roughness is difficult, especially on purely ceramic substrates, however it can be accomplished if the layer does not require a strong bond to the substrate or the substrate exhibits better Si wetting properties. Electrical resistivity of less than 5 $\mathrm{m} \Omega \cdot \mathrm{cm}$ has been demonstrated, while thermal conductivity measurements of $1.36 \mathrm{~W} / \mathrm{m} / \mathrm{K}$ at room temperature validate the goal of this research to push $\kappa_{\mathrm{ph}}$ nearer to the amorphous limit of $\sim 1 \mathrm{~W} / \mathrm{m} / \mathrm{K}$.

\subsection{Material Performance and Efficiency (ZT and $\eta$ )}

While the laser method successfully sintered the nanocluster films, dilution into the $\mathrm{Si}$ substrate results in a non-homogeneous distribution of composition. The electrical measurements themselves are considered accurate, likewise for the thermal measurements, although the two data sets don't measure the same volume of the sample and thus integrating the results is not an accurate model of performance. These two datasets are shown for one n-type and one p-type sample in Figure 6.46. Only if the thermal data were an average of the entire conducting thickness would the calculation be logical, instead of just the top $\sim 300-400 \mathrm{~nm}$ of the sintered layer. Given that this data is not available and just to show the steps, the electrical data will be integrated assuming a static thermal conductivity of $20 \mathrm{~W} / \mathrm{m} / \mathrm{K}$. This value is about 3 times worse than what was used by NASA for the RTG system and is a simple target to meet with any $\mathrm{Si}-\mathrm{Ge}$ system of more than a few at\% Ge [23]. Thus, in a projection using the assumed thermal conductivity of $20 \mathrm{~W} / \mathrm{m} / \mathrm{K}$, the value of ZT for both the $n$-type and p-type materials are calculated in Figure 6.47. The projected average ZT from 300 to $1000 \mathrm{~K}$ for the n-sample would be 0.074 with an efficiency of $1.9 \%$. The projected average ZT from 300 to $800 \mathrm{~K}$ for the p-sample would be 0.004 with an efficiency of $0.1 \%$. Actual material figure of merit should be able to exceed these projections, which is left as future work. 


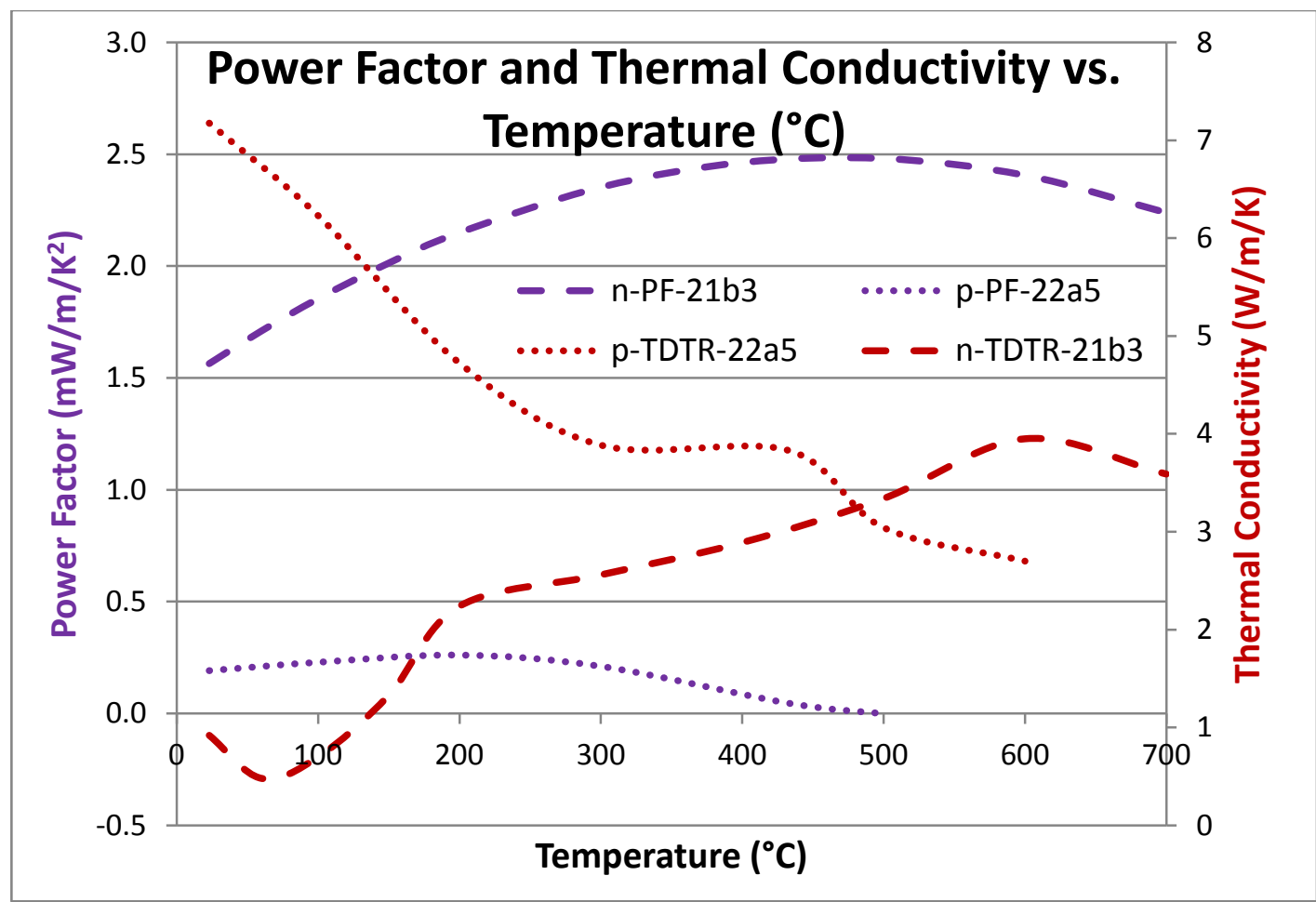

Figure 6.46. Measured power factor and thermal conductivity for a n- and p- type sample. The Si substrate allowed significant dopant diffusion and some Ge diffusion from the surface, such that the electrical and thermal performances are not directly comparable.

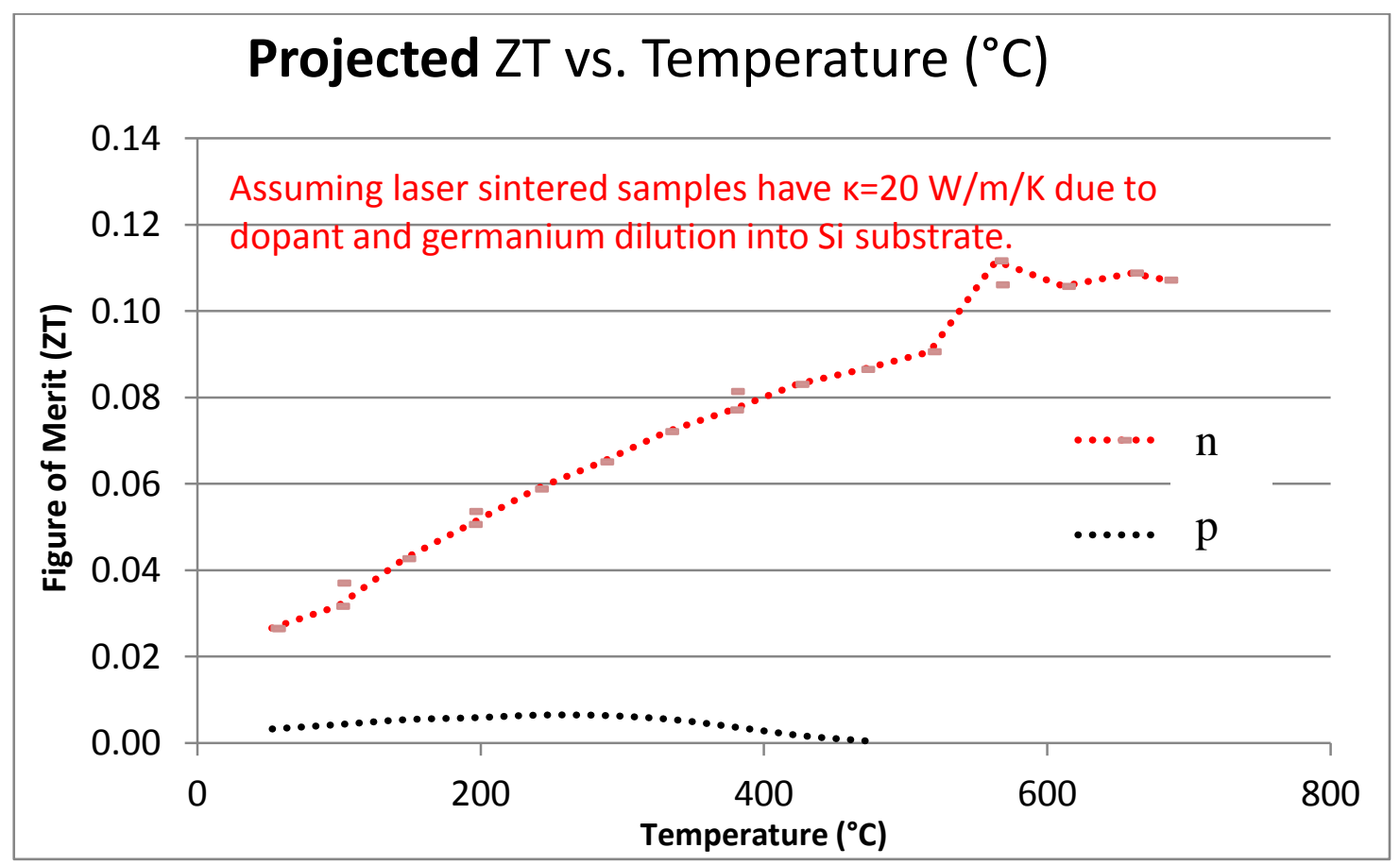

Figure 6.47. ZT projection for two laser sintered samples, given Si substrate dilution. 


\section{Chapter 7 : Additional Research Related to Thermoelectrics}

\subsection{Sintering of Si-Ge Powders Using 940 nm CW Laser}

\subsubsection{Process Parameters}

Laser sintering of Si-Ge was investigated with micro- and nano-powders as a first order feasibility study. After creating a powder composition of $\mathrm{Si}_{0.79} \mathrm{Ge}_{0.2} \mathrm{~B}_{0.01}$ and dispersing onto a substrate, laser treatment using the $940 \mathrm{~nm} \mathrm{CW}$ laser was applied at various conditions. The laser intensity was varied between 2 and $320 \mathrm{~W} / \mathrm{mm}^{2}$ and the scan speed varied from 0.5 to 6 $\mathrm{mm} / \mathrm{s}$, with the highest powers used when working with graphite substrates. Multiple passes were often used to minimize evaporation of materials, where laser intensity was increased with each successive pass; 3 passes was the maximum attempted. Likewise, the laser spot size was also adjusted up to $2.5 \mathrm{~mm}$ diameter to assist with densification of the thick layers before reaching the evaporation threshold.

When using $\sim 800{ }^{\circ} \mathrm{C}$ preheating temperature (300 amps I.H.) combined with very high laser intensities of $\sim 150 \mathrm{~W} / \mathrm{mm}^{2}$, it was possible to sinter films with thickness greater than 200 $\mu \mathrm{m}$. SEM images of these results show grains with sizes around $10 \mu \mathrm{m}$, but the crystallite size is not known. Presumably nanofeatures would be lost under such high temperatures, however the excellent densification does demonstrate feasibility of the laser sintering process with $\mathrm{Si}_{0.8} \mathrm{Ge}_{0.2}$.

All reported powder sintering results here use the nano-size powder on a molybdenum substrate. The powders are described in section 4.1.1.

\subsubsection{Morphology and Composition}

Successful laser sintering has been accomplished using silicon germanium on both metal and ceramic substrates using a diode laser. Figure 7.1 shows two SEM images of $\mathrm{Si}_{0.79} \mathrm{Ge}_{0.20} \mathrm{~B}_{0.01}$ laser sintered on a molybdenum substrate. The left image shows the general morphology of the sintered film, with good densification and only moderate surface height variation. The right images shows clusters of sintered particles (one such cluster is circled in red), but each cluster is composed of many nanocrystallites. 


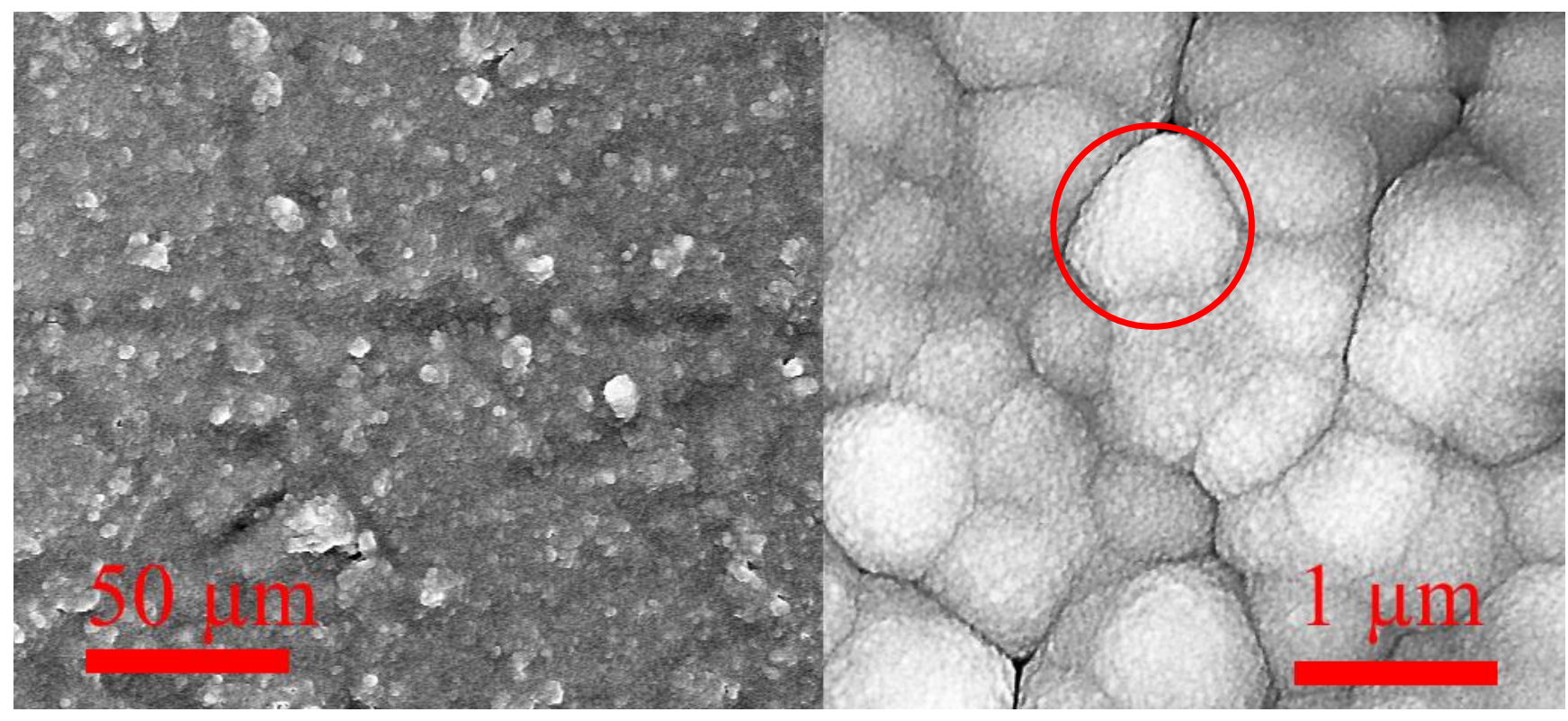

Figure 7.1. SEM of laser sintered Si-Ge nanopowder.

The circle highlights one cluster of nanocrystallites.

The composition of this sample was analyzed using Energy Dispersive $x$-ray Spectroscopy (EDS) as shown in Figure 7.2. EDS showed a large concentration of oxygen, indicating significant oxidation of the sample surface during processing. The boron concentration was not high enough to resolve using EDS, and the molybdenum impurity may result from porosity in the sample allowing the EDS electron beam to penetrate to the substrate. Also of note was the slightly lower ratio of $\mathrm{Ge}$ to $\mathrm{Si}$, which was often true using EDS of $\mathrm{Si}_{0.8} \mathrm{Ge}_{0.2}$ and thus this discrepancy is primarily ascribed to using uncalibrated analysis, although some Ge loss during laser heating could certainly be a factor. Ge loss may occur due to both germanium's lower melting point as well as preferential laser heating due to the much shorter laser absorption depth ( 100 times smaller than Si for green to near-IR light), as illustrated in Figure 6.14. 


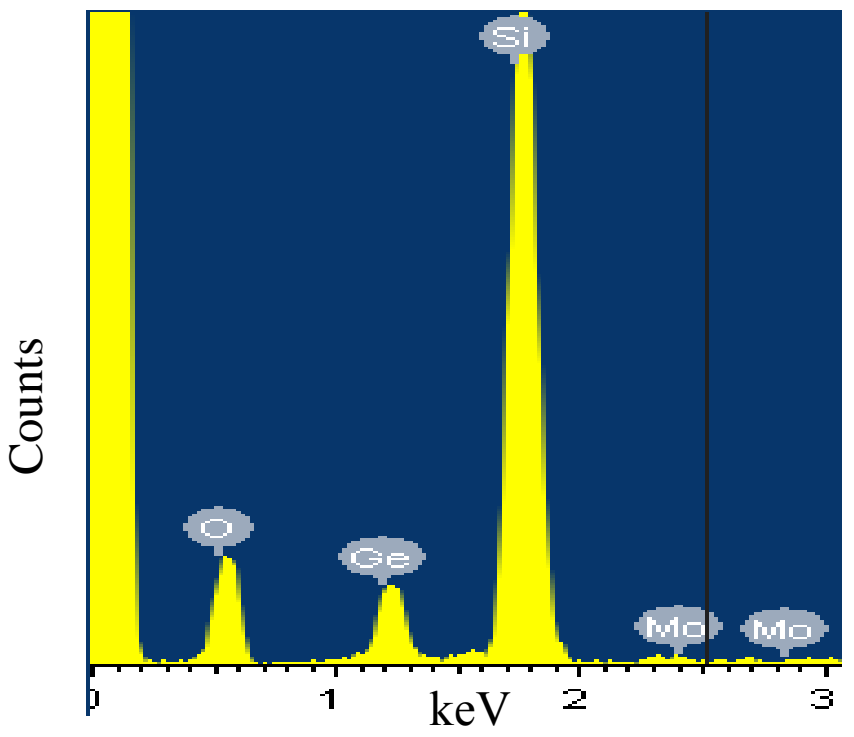

\begin{tabular}{|c|c|}
\hline SEM & $\begin{array}{c}\text { Uncalibrated } \\
\text { at } \%\end{array}$ \\
\hline Si K & 39 \\
Ge L & 5 \\
O K & 56 \\
Mo L & 0.4 \\
\hline
\end{tabular}

Figure 7.2. Si-Ge nanopowder composition by EDS in SEM after sintering by $940 \mathrm{~nm}$ laser.

\subsubsection{Crystal Structure Characterization}

The raw nanopowder XRD results were presented in Figure 6.2 and induction heated XRD results in Figure 6.3. The XRD result in Figure 7.3 is for a laser sintered sample.

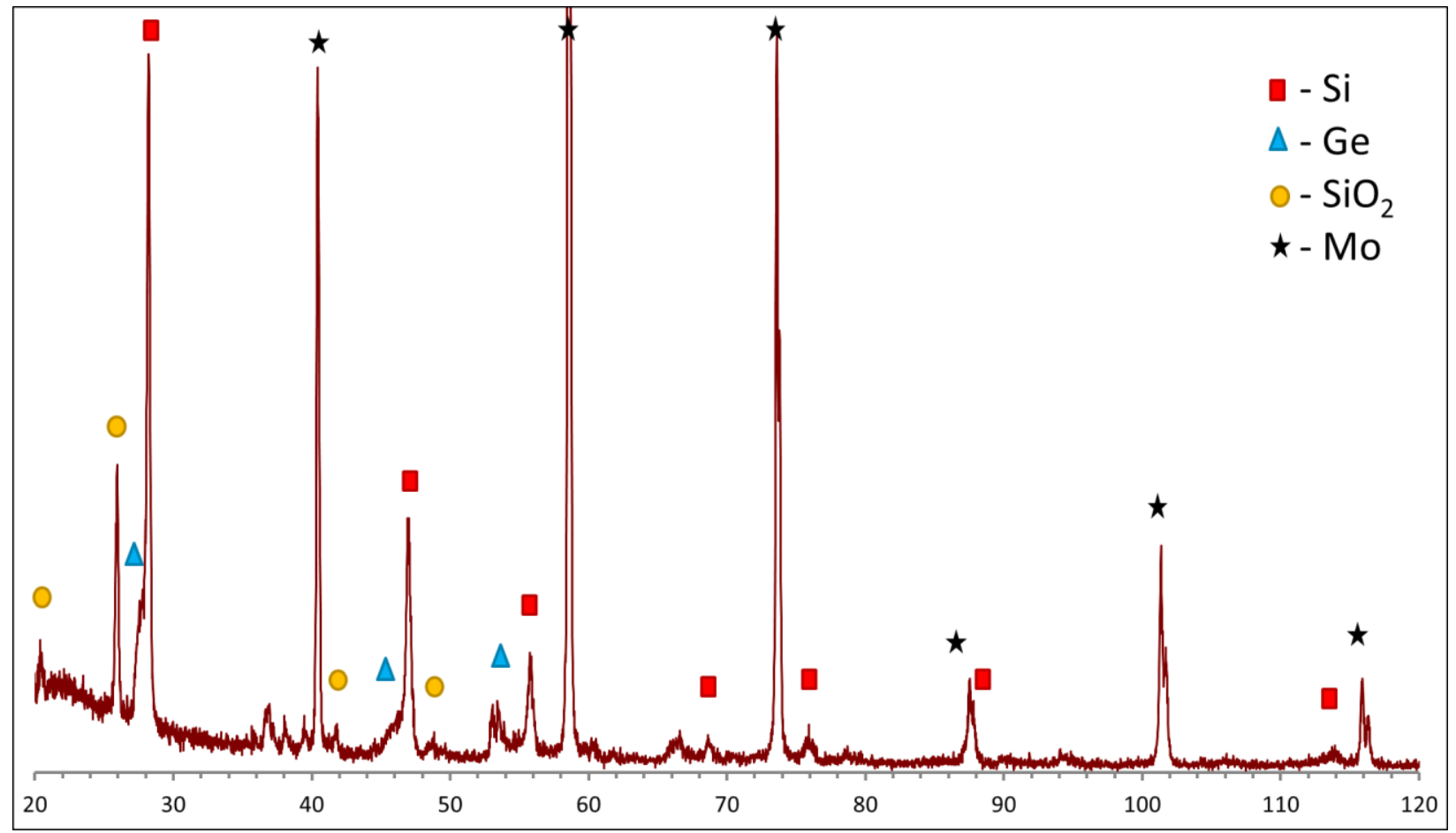

Figure 7.3. XRD phase analysis of Si-Ge nanopowder after laser sintering on Mo substrate. 
XRD results of laser sintering on molybdenum substrate show significant broadening at the shoulder of the Si peaks, where the Ge diffused into the Si lattice forming an alloy with a lattice parameter up to $4 \%$ larger. The Scherrer equation was used to determine the crystallite size from peak broadening, calculated as $\sim 32 \mathrm{~nm}$ neglecting instrumental broadening and compositional variation, suggesting either A) the original nanoparticles were not crystalline since $32 \mathrm{~nm}$ is less than the $50 \mathrm{~nm}$ size specification or B) the hand milling was effective in crushing the particles to smaller size. If the alloy was not homogenous then this grain size analysis may underestimate the true crystallite size, however the distribution of lattice spacing should still assist the goal of phonon scattering.

\subsubsection{Summary}

For actual application, obtaining adhesion to the typical molybdenum and tungsten substrates by laser sintering is significantly less challenging than working with ceramic substrates. Working with larger powder particles allows sintering thicker layers, but not necessarily while maintaining an extremely high density of grain interfaces. The XRD measurements of processed nanopowders support the conclusion of low grain growth during laser sintering.

\subsection{Sintering of Si-Ge Thin Films Using Rapid Thermal Anneal}

\subsubsection{Process Parameters}

As an alternative to laser sintering, Rapid Thermal Anneal (RTA) was employed to try to create a smooth and continuous sintered film using $5 \mathrm{~nm}$ doped $\mathrm{Si}-\mathrm{Ge}$ nanoclusters provided by UMN. The RTA (AS-One 100, Annealsys, France) can heat up to $1100{ }^{\circ} \mathrm{C}$ by applying up to 30 $\mathrm{kW}(100 \%)$ of heat by thermal radiation. An example of the heating profile is given in Figure

7.4. Hold times of 1 minute to 10 minutes were tested, using a hold temperature range of 850 to $1100{ }^{\circ} \mathrm{C}$. To prevent oxidation during annealing, the chamber was vacuum pumped to $3 * 10^{-5}$ Torr and back filled with gas three times, and then filled to 1 ATM before heating. Gases used included nitrogen, forming gas, and ultimately argon. A fused silica cover plate was used to try to reduce contamination from the chamber during the annealing process, however if the sample 
was heated too fast the cover would be blown off the sample due to hydrogen evolution. The cooling ramp rate was also reduced to mitigate film cracking and delamination upon cooling back to room temperature.

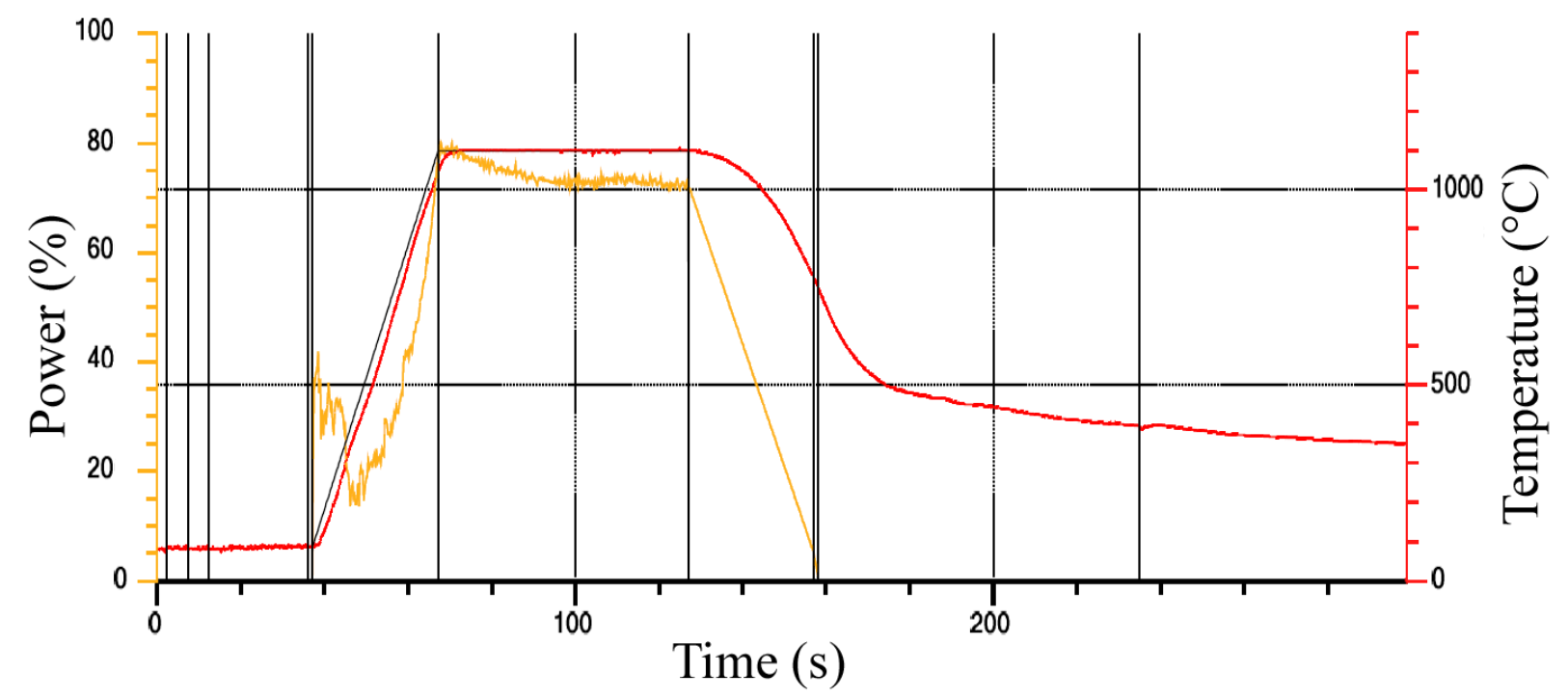

Figure 7.4. RTA example of heating profile vs. time.

\subsubsection{Morphology}

The thin films did not uphold well inside the RTA, typically having insufficient sintering to create a continuous film and thus lacked good electrical conductivity. At lower temperature and/or duration settings (e.g., $<1000{ }^{\circ} \mathrm{C}$ or $<2$ minutes hold time), there was insufficient time to provide adequate sintering and thus densification was not complete as in Figure 7.5. If the temperature ramp rate was too fast, the temperature would overshoot significantly and could boil the surface of the nanocluster film, which would leave the surface covered with exploded bubbles as in Figure 7.6. It was determined that the ramp rate of $1000{ }^{\circ} \mathrm{C}$ per minute offered a good balance of densification without damaging the surface. Improving the process required annealing with a higher temperature (1050-1100 ${ }^{\circ} \mathrm{C}$ ) and medium hold times (4-10 minutes). In this case, the surface of the film exhibited the desired necking and grain growth during sintering as in Figure 7.7, however the macro-morphology still showed cracking, delamination or other inconsistencies. Increasing the anneal duration resulted in contamination from the chamber as noticed when analyzing the composition with EDS. 

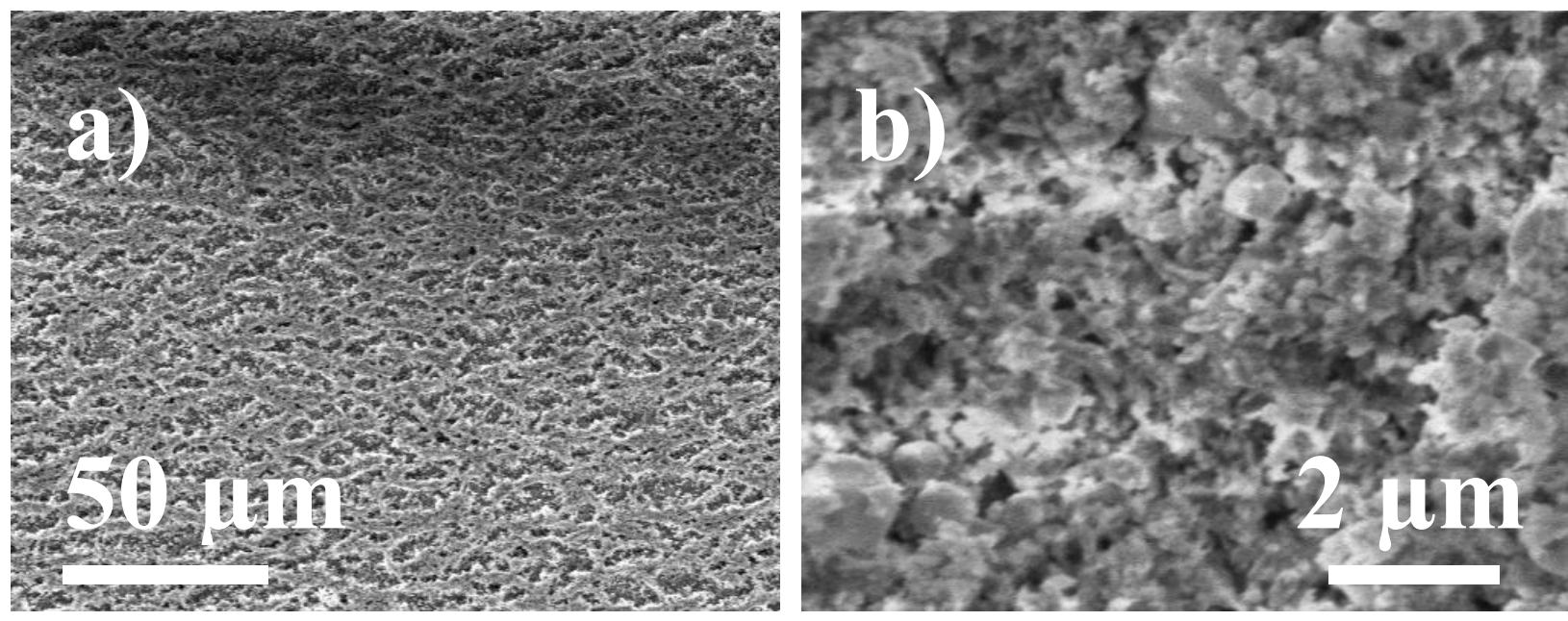

Figure 7.5. Surface film morphology showing porosity using $1075^{\circ} \mathrm{C}$ RTA with slow ramping. a) low magnification, b) high magnification

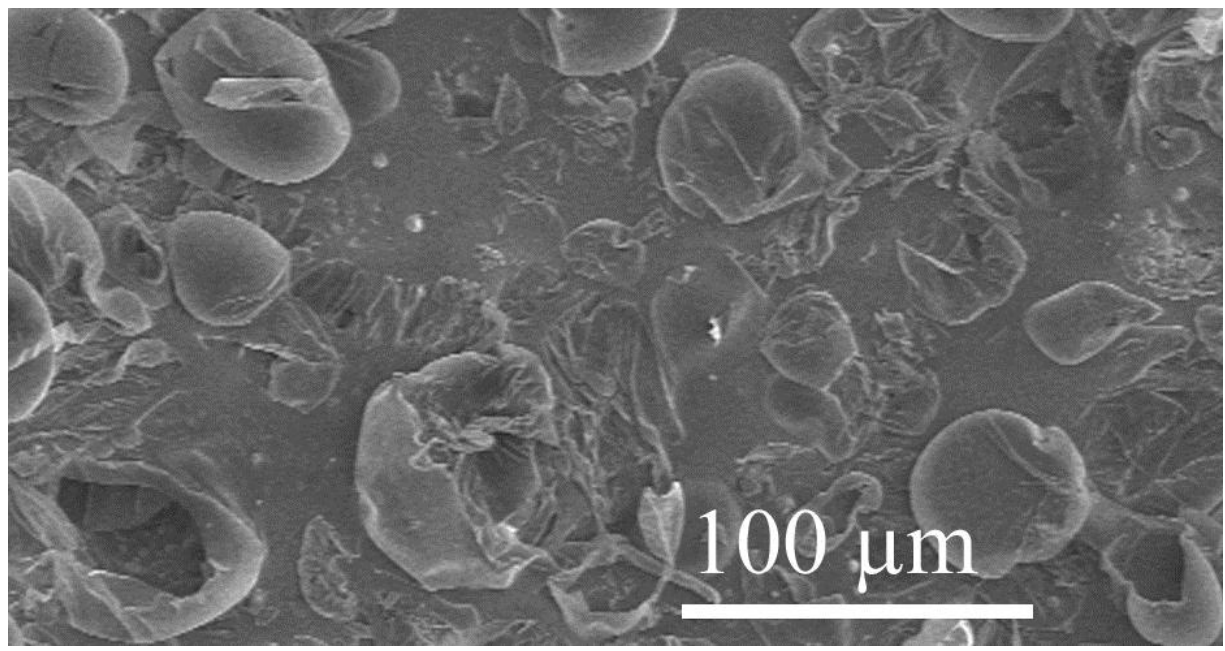

Figure 7.6. Surface film morphology using RTA to $1100^{\circ} \mathrm{C}$, with boiling from fast heating rate. 


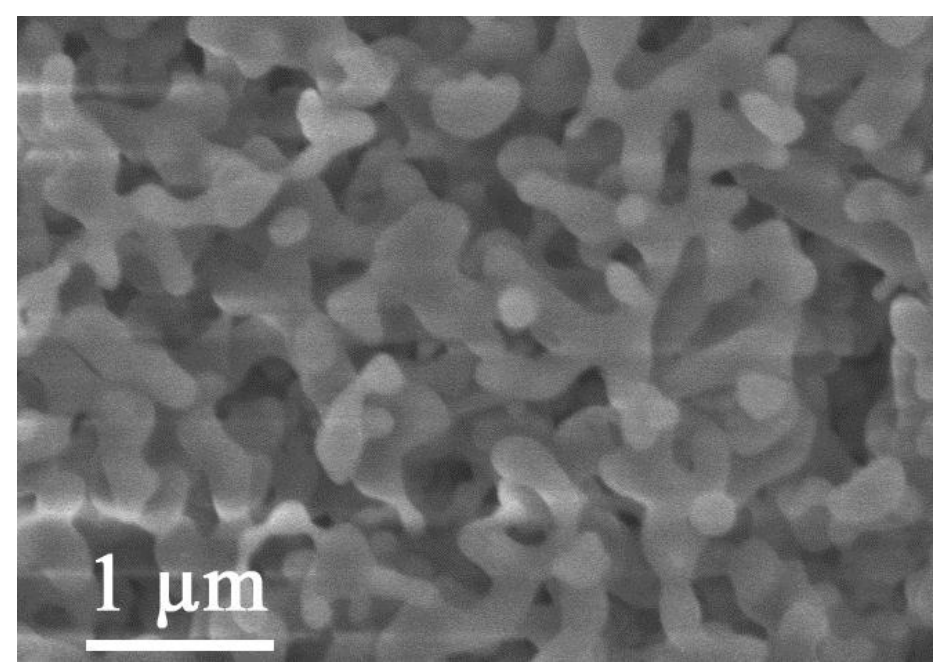

Figure 7.7. Surface film morphology using RTA to $1100^{\circ} \mathrm{C}$, with improved sintering.

\subsubsection{Composition}

SEM/EDS showed that often the RTA was contaminating the samples with lighter elements such as nitrogen and perhaps carbon, despite the sample being processed in argon. As the RTA is shared with many users and isn't normally operated above $900^{\circ} \mathrm{C}$, the outgassing of contaminants may have adversely impacted the sample morphology and conductivity.

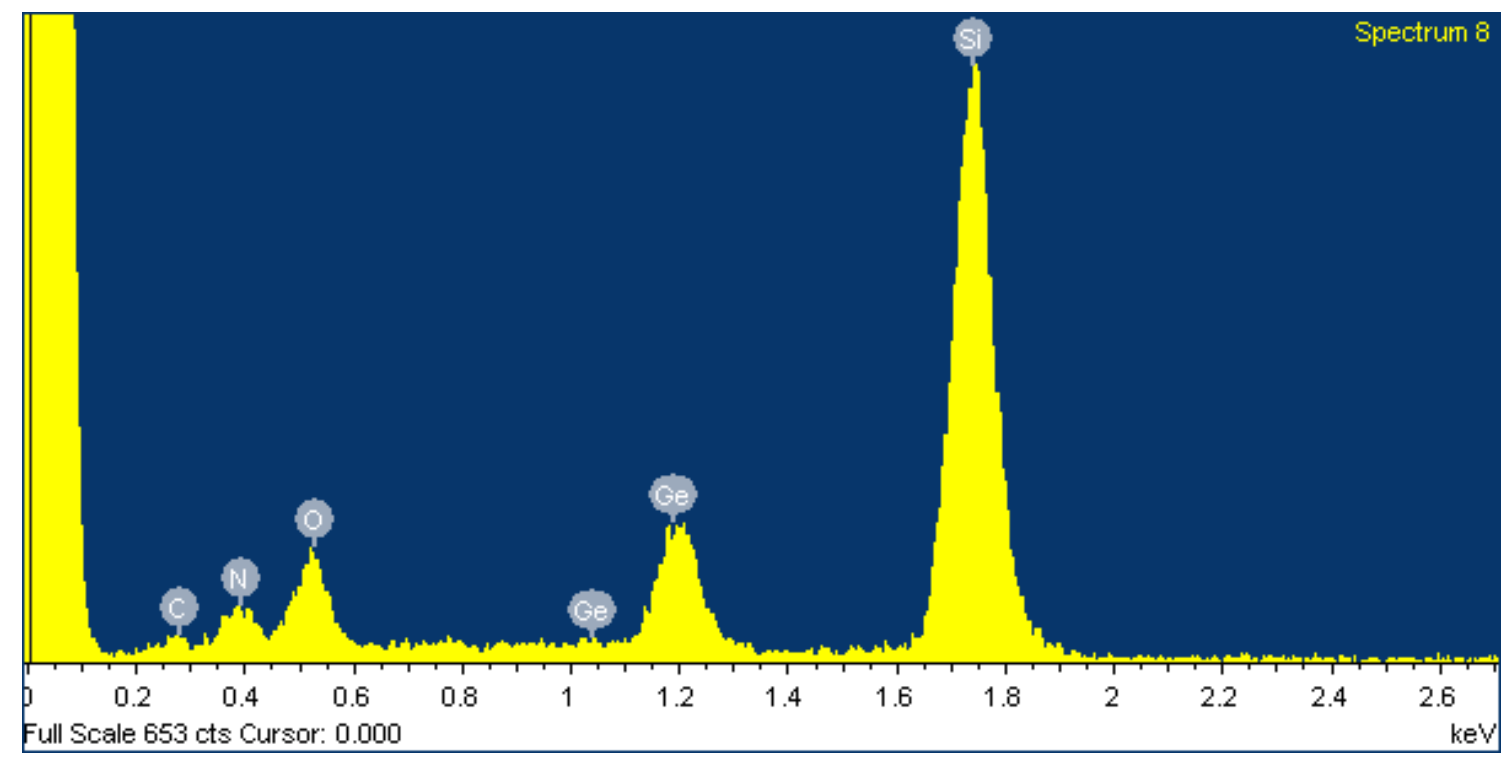

Figure 7.8. Composition when using RTA, exhibiting nitrogen contamination from the chamber. 


\subsubsection{Summary}

While film electrical conductivity was improved by orders of magnitude during the RTA process, resistivity below $\sim 10^{5} \Omega \cdot \mathrm{cm}$ was not achieved. Moreover, the RTA was not able to provide good densification without sample contamination, and strain due to thermal expansion mismatch also caused film discontinuities. Therefore, research into annealing by the RTA method was abandoned in favor of laser treatment.

\subsection{Sintering of Si-Ge Thin Films Using 532 nm CW Laser}

Laser sintering was also investigated using an $18 \mathrm{~W}, 532 \mathrm{~nm} \mathrm{CW}$ laser. The sintering was performed in vacuum, with no option for preheating. Testing over a range of laser powers and scan speeds did not yield a conductive film, and brief analysis using SEM indicated the laser treatment did not offer good film morphology. This quality issue likely resulted from the fact that the laser absorption depth for Ge at $532 \mathrm{~nm}$ wavelength is very short, much shallower than the depth of the film. Moreover, the lack of ability to preheat the sample using this experimental setup limited the ability to create an integral bond with the substrate while simultaneously maintaining percolation. Therefore, it was concluded that since this research used films significantly thicker than one micron, this laser would not provide optimal conditions for sintering of $\mathrm{Si}_{0.8} \mathrm{Ge}_{0.2}$ so further investigation was discontinued in favor of using the $940 \mathrm{~nm}$ laser. 


\section{Chapter 8 : Additional Research Related to Laser Processing of Materials}

\subsection{Microwave + Laser Hybrid Sintering of $\mathrm{ZrB}_{2}$}

"Fabrication of ZrB2-Zr cermet using laser sintering technique"

C. N. Sun, T. Baldridge and M. C. Gupta, Mater. Lett., v 63, pp. 2529-2531 (2009).

The Ultra High Temperature Ceramic (UHTC) $\mathrm{ZrB}_{2}$ has potential aerospace application for the protection of leading edges on atmospheric reentry vehicles due to high chemical and thermal stability along with high thermal conductivity. The high thermal conductivity permits sharper leading edges by spreading the heat generated from high-speed flight to cooler areas. Yet, processing of $\mathrm{ZrB}_{2}$ remains challenging due to the extremely high melting point and very high hardness. In this research, simultaneous application of microwave and laser power was investigated for sintering a powder of $\mathrm{ZrB}_{2}$ into a continuous layer. As the dielectric constant of $\mathrm{ZrB}_{2}$ at $2.4 \mathrm{GHz}$ was unknown, this work investigated whether $\mathrm{ZrB}_{2}$ would act as a susceptor to enable volumetric heating typical of microwaves. While solid metals tend to reflect microwaves, a powder should allow deeper penetration as the radiation scatters at the particle boundaries. Likewise, the oscillating field could directly heat the particles from electron movement. Using the microwave alone, however, did not result in significant heating of the powder, and it was determined that the $\mathrm{ZrB}_{2}$ did not undergo volumetric heating until it was first preheated to more than a few hundred degrees Celsius. A microwave chamber was modified to allow entry of a laser beam to heat the surface of the sample, as shown in Figure 8.1, and additional heat was provided by adding $\mathrm{SiC}$ susceptors near the sample. These modifications allowed initial sintering of the sample, but oxidation during processing converted much of the $\mathrm{ZrB}_{2}$ into a thick surface layer of zirconia. Oxidation control was attempted by filling a chamber inside the microwave with argon, but the breakdown voltage of Ar is much lower than that of air, and thus the Ar facilitated arcing which consumed enough of the microwave power to prevent sintering of the sample. The results of this work led to further research on sintering $\mathrm{ZrB}_{2}$ with a laser in combination with preheating by an induction heater. Good densification and morphology was obtainable through the addition of refractory metals to the $\mathrm{ZrB}_{2}$. 


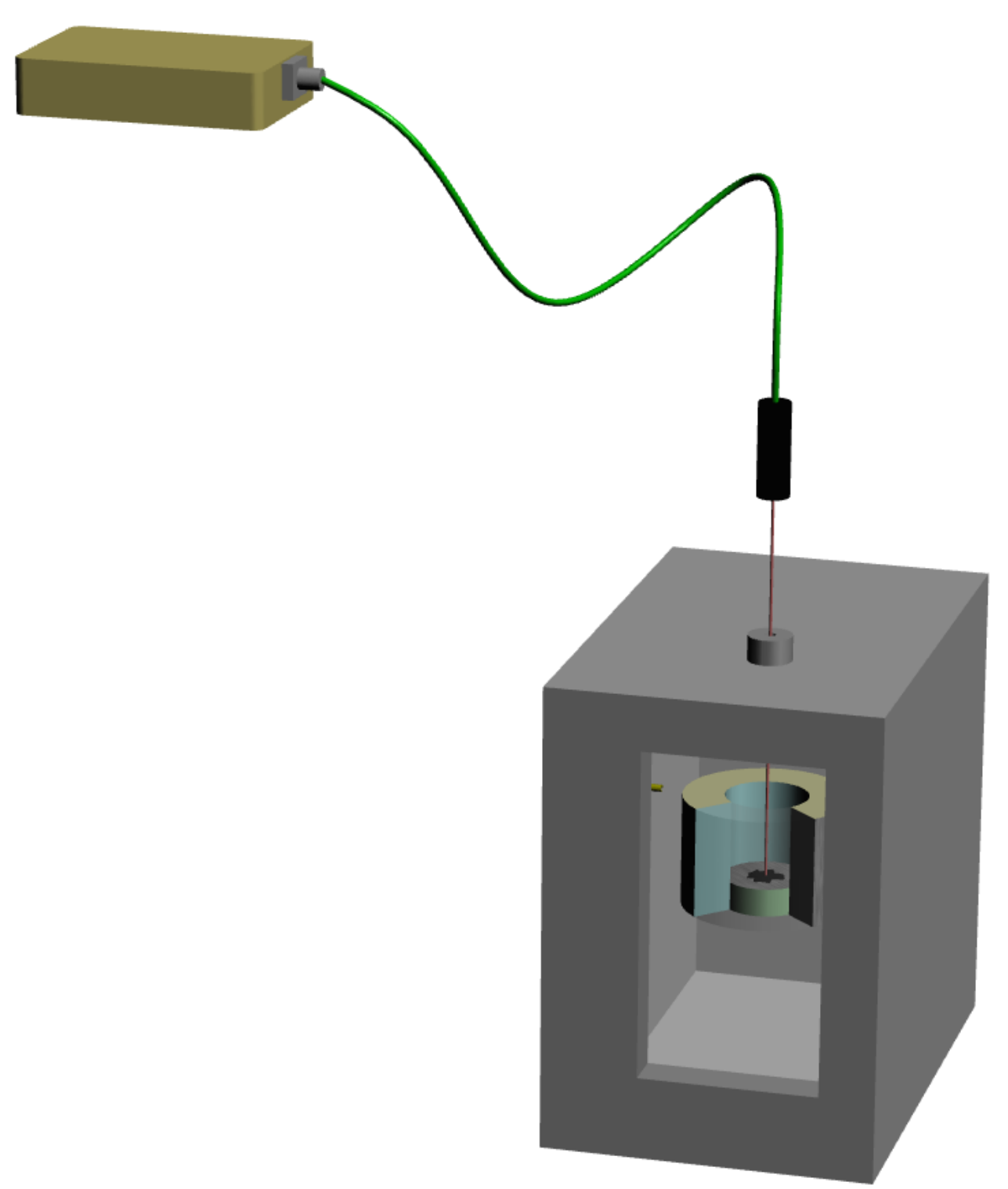

Figure 8.1. Experimental setup for microwave + laser hybrid sintering.

\subsection{Nanostructure Generation via Microwave Arc Heating of $\mathrm{ZrB}_{2}$}

"Nanostructures from Zirconium Diboride and Alumina Ceramics"

T. Baldridge, M. C. Gupta, and C. N. Sun, J. Am. Ceram. Soc., v 9, pp. 2891-2896 (2010).

"Zirconium diboride nanofiber generation via microwave arc heating"

T. Baldridge and M. C. Gupta, Nanotechnology, v 19, pp. 275601-275607 (2008).

The UHTC zirconium diboride has a melting point of $>3000{ }^{\circ} \mathrm{C}$. It has mechanical properties similar to ceramics, but has high thermal and electrical conductivity similar to iron or lead. In this research, powders of zirconium diboride were exposed to $2.4 \mathrm{GHz}$ microwave 
radiation. Under specific conditions, the large applied field caused the movement of electrons between powder particles, resulting in arcing throughout the powder. The arcing generates tremendous localized heating to the point of plasma formation, with a duration on the order of seconds. The extremely fast heating and cooling rates of the materials facilitated the formation of unique nanostructures. Most notable was the generation of rectangular nanotubes at the powder to substrate interface. The alumina substrate reacted with the vaporized $\mathrm{ZrB}_{2}$ particles to form tubes with average dimensions of $\sim 5 \times 1 \times 1 \mu \mathrm{m}$. These tubes were characterized by SEM, EDS, AES, TEM, EELS, and SAED, and concluded to be most similar to a boron-rich mullite with zirconium impurity.

\subsection{Laser Cladding of Inconel 690 Superalloy}

\section{"Inconel 690 laser cladding on Inconel 600 superalloy for corrosion protection in nuclear applications"}

T. Baldridge, G. Poling, E. Foroozmehr, R. Kovacevic, T. Metz, V. Kadekar, and M.C. Gupta, Optics and Lasers in Engineering, v 51, i 2, pp. 180-184.

In the nuclear industry there is need for repair of heat exchanger tubes made of hightemperature corrosion-resistant Inconel metals. This work reports the results of applying a $3 \mathrm{~mm}$ thick cladding layer by laser melting Inconel 690 powder on top of a $10 \mathrm{~mm}$ thick plate of Inconel 600 metal. Successful multilayer cladding of $3 \mathrm{~mm}$ thickness was achieved by scanning the laser beam over the substrate using a powder feeder to control the powder feed rate. Experimental parameters such as laser power, scanning speed, beam overlap, powder flow rate, and preheating were investigated to reduce ductility-dip cracking upon cooling. SEM images show a smooth integral interface between the 600 and 690 materials, and EDS mapping reveals the dilution zone via the concentration gradient of chromium. Vickers tests show the 690 cladding surface to be up to $40 \%$ harder than the base 600 material. XRD and EDS analysis confirm that the Inconel 690 composition remains unchanged throughout processing when using argon as a shielding gas. The final laser melted cladding layer appears to be well-suited for surface protection. 


\section{Chapter 9 : Conclusion and Future Plan}

\subsection{Conclusion}

For the first time, continuous-wave laser sintering has been successfully demonstrated for the production of silicon-germanium thermoelectric applications. The motivation was to achieve lowered thermal conductivity via a high density of nano-grain interfaces, enabled by starting with 5-9 $\mathrm{nm}$ nanoclusters in combination with fast laser heating rates. Compared with state of the art bulk $\mathrm{Si}-\mathrm{Ge}$, the results presented here provide a tremendous reduction in the thermal conductivity of $24 \%$ lower. Reducing thermal conductivity of the Si-Ge alloy system to the amorphous limit of $\sim 1 \mathrm{~W} / \mathrm{m} / \mathrm{K}$ could increase ZT by $\sim 25 \%$ and improve efficiency by $\sim 20 \%$ compared with current state of the art Si-Ge materials [78]. This work has successfully demonstrated laser sintering of doped Si-Ge powders and nanoclusters into thin film thermoelectric materials. Next we describe some of the major accomplishments and conclusions from this research investigation.

A. Collaboration was established with Prof Kortshagen's group at the University of Minnesota at the Fall 2010 Materials Research Society conference on thermoelectrics. This partnership enabled the acquisition of doped silicon germanium nanoclusters as small as $5 \mathrm{~nm}$, which were doped and alloyed on the scale of the particle size. These types of materials are otherwise unavailable, yet a perfect fit for reaching the amorphous limit of thermal conductivity for Si-Ge. Furthermore, our proposal for characterization of laser sintered thermoelectric materials was approved by Oak Ridge National Laboratory. Thus, the facilities at the ORNL High Temperature Materials Laboratory (HTML) were made available to characterize the high temperature thermoelectric performance of the raw nanoclusters and laser sintered films. Finally, a collaborative effort with Prof. Patrick Hopkins at the University of Virginia enabled both low and high temperature thermal conductivity measurements of the Si-Ge films using Time-Domain ThermoReflectance (TDTR).

B. An experimental setup was created which enabled high temperature preheating and laser sintering of the thermoelectric films. The chamber design contains an induction coil, which in combination with a Mo or $\mathrm{W}$ substrate, allows heating materials to over $1000{ }^{\circ} \mathrm{C}$ in a 
protected environment such as argon or nitrogen. The chamber is mounted to a computer controlled X-Y stage to allow scanning the laser beam over a sample area of $25 \mathrm{~cm}^{2}$; without utilizing induction heating the laser can be scanned over a $100 \mathrm{~cm}^{2}$ area. The creation of this chamber overcame the previous limitations exhibited due to heavy oxidation of many materials at high temperatures.

C. Nanocluster thin films of $\mathrm{Si}_{0.8} \mathrm{Ge}_{0.2}$ were characterized to understand their morphology, structure, stability, composition, consistency, and thickness. These properties were analyzed using SEM, TEM, EDS, XRD, and optical microscope, and the understanding gained from this characterization enabled laser sintering the films into a semi-continuous percolating network.

D. Thermal conductivity measurements validated the achievement of one of the lowest roomtemperature thermal conductivity values reported for the Si-Ge system. The TDTR results show that we have realized the research goal of reducing thermal conductivity by taking a bottom-up approach which used laser sintering of $9 \mathrm{~nm}$ doped $\mathrm{Si}$-Ge nanoclusters to produce a film with a high density of interfaces for enhanced phonon scattering. The measured room temperature thermal conductivity was $1.36 \mathrm{~W} / \mathrm{m} / \mathrm{K}$, significantly lower than the previous best of $1.78 \mathrm{~W} / \mathrm{m} / \mathrm{K}$ by Wang et al. [78] and approaching the amorphous limit of Si-Ge [24]. At high temperatures the thermal conductivity was still quite low ( 2 to $8 \mathrm{~W} / \mathrm{m} / \mathrm{K}$ ), but not as significant as the results below $200{ }^{\circ} \mathrm{C}$. Further research is necessary to understand the mechanism of increasing thermal conductivity above this temperature.

E. Electrical resistivity was heavily dependent upon the morphology of the sintered layer, which was in turn dependent upon the substrate material. Depending upon the substrate used, the best achieved results at room temperature were:

- fused silica : $\sim 0.025 \Omega \cdot \mathrm{cm}$

- silicon: $\quad \sim 0.004 \Omega \cdot \mathrm{cm}$

- silicon carbide: $\sim 0.005 \Omega \cdot \mathrm{cm}$

The electrical resistivity needs to be further reduced by a factor of $\sim 5$ to compete with bulk values. The Ulvac ZEM-3 measurements from room temperature to $1000 \mathrm{~K}$ show a high power factor at low temperatures and a reduced power factor at high temperatures. Reaching 
the ideal doping concentration of $2 * 10^{20} / \mathrm{cm}^{3}$ would significantly improve the electrical performance of the material.

F. XRD measurements support that the crystallite size of laser sintered Si-Ge nanoclusters on $\mathrm{Si}$ substrate remained on the nano-scale, near $\sim 25 \mathrm{~nm}$. High-temperature XRD scans confirm the laser sintered nanocluster films are stable to $1000{ }^{\circ} \mathrm{C}$, the typical peak operating temperature for Si-Ge. Additional XRD analysis of raw and preheated nanocluster crystallite size confirms the initial clusters are $\sim 9 \mathrm{~nm}$ before and after preheating to $\sim 800{ }^{\circ} \mathrm{C}$ for eight minutes, thus preheating can be used to improve film morphology without concern of increased thermal conductivity. On the other hand, $5 \mathrm{~nm} \mathrm{Si-Ge} \mathrm{nanoclusters} \mathrm{experienced}$ grain growth by $600{ }^{\circ} \mathrm{C}$ with long heating times (e.g., one hour).

G. Optimization of the laser sintering process and nanocluster material specification was accomplished through continuous feedback from characterization methods such as electrical resistivity and mobility, optical microscope, thermopower, SEM, TEM, EDS, XRD, and TDTR. In combination, these characterization methods provided insight into the laser sintering process, allowing improved processing parameters and providing scientific understanding of the laser sintering process with thin films of nanoclusters. Some of the observations included:

a. $\quad$ significant depression of the melting point to $\sim 600^{\circ} \mathrm{C}$ for nanoclusters of $5 \mathrm{~nm}$

b. $9 \mathrm{~nm}$ nanoclusters did not exhibit grain growth to over $800{ }^{\circ} \mathrm{C}$

c. effective doping level for the laser sintered films on Si substrate was $\sim 1 * 10^{19} / \mathrm{cm}^{3}$

d. mobilities for n-type samples decreased from 77 to $300 \mathrm{~K}\left(\sim 120 \rightarrow 80 \mathrm{~cm}^{2} / \mathrm{V} / \mathrm{s}\right)$, whereas p-type samples had similar mobilities at both temperatures $\left(\sim 40 \mathrm{~cm}^{2} / \mathrm{V} / \mathrm{s}\right)$

e. under certain conditions, laser sintered thin films achieved integral bonds with the substrates, and could develop percolating networks with good electrical conductivity

\subsection{Suggestions for Future Investigations}

Characterization of laser sintered films on silicon carbide substrate will better enable comparison of performance to NASA RTG and state of the art results. High temperature 
measurement of the silicon carbide substrate samples which have already been laser sintered should remove the non-homogeneity between thermal and electrical analysis such that ZT and $\eta$ can be confidently calculated.

Laser sintering of thin films of Si-Ge nanoclusters requires further optimization to the processing parameters to improve electrical conductivity. Process modifications to be investigated include:

studying initial nanocluster size impact on achieving good film morphology and electrical conductivity

$>$ studying crystallite and grain size effect on $\kappa, \sigma$, and $\mathrm{S}$ to optimize overall performance

$>$ using nanocluster films of different thicknesses for enhanced morphology, such that the depth of where the majority of energy absorption occurs can be shifted

testing other low-bandgap ceramics like $\mathrm{Si}_{3} \mathrm{~N}_{4}$ to improve substrate bonding

$>$ depositing a germanium layer before the Si-Ge layer to facilitate substrate bonding and wetting, to reduce preferential Ge light absorption, and reduce mass losses during laser processing

$>$ using nano-inclusions such as $\mathrm{GaP}$ to improve dopant density and phonon scattering

$>$ depositing overdoped nanoclusters $\left(\mathrm{Si}_{0.8} \mathrm{Ge}_{0.2} \mathrm{~B}_{0.05}\right)$ with undoped $\mathrm{Si}-\mathrm{Ge}$ to investigate the benefit of modulation doping

$>$ using a pulsed laser to provide high peak intensity which may catalyze surface adhesion.

$>$ using a $1064 \mathrm{~nm}$ or longer wavelength laser as the deeper power absorption may catalyze substrate adhesion without ablation.

Ultimately, the ideal configuring would be for laser sintering to occur inside the plasma deposition chamber, such that thick films can be constructed without oxidation and with a constant supply of particles for full densification. Furthermore, in situ sintering would enable functional grading of both the Si:Ge ratio and the dopant concentration. Functional grading could moderately improve the figure of merit of Si-Ge, especially for applications at temperatures near 300 or $1300 \mathrm{~K}$, where the ideal dopant concentration significantly varies from the average ideal of $2 * 10^{20} / \mathrm{cm}^{3}$. 
Successful implementation of thermoelectric generation (TEG) could evolve mobile power production (e.g., automobiles) or remove the need for new power plant construction by locally generating power at some of the highest-intensity energy users such as incinerators and refineries.

In conclusion, this work proved that nanograined thin films of $\mathrm{Si}-\mathrm{Ge}$ can be laser sintered to obtain good electrical conductivity and excellent thermal conductivity. Additional investigation is needed to characterize non-diluted thin films to high temperature such that ZT can be accurately calculated. Likewise, further scientific studies testing the proposed process modifications above should assist in taking the next step in optimizing this material system for high-temperature thermoelectric applications. The results here facilitate future research on $\mathrm{CW}$ laser sintering of Si-Ge, and may be useful to other high operating temperature thermoelectric materials which need nanograins to further reduce thermal conductivity. This work serves as a foundation for continuous-wave laser sintering of $\mathrm{Si}-\mathrm{Ge}$ or other thermoelectric materials. 


\section{References}

[1] J.-P. Fleurial, P.K. Gogna, B.-Y. Li, S. Firdosy, B.J. Chen, C.-K. Huang, V. Ravi, T.

Caillat, and K. Star, "Waste heat recovery opportunities for thermoelectric generators," Sep. 2009, Available:

http://www1.eere.energy.gov/vehiclesandfuels/pdfs/thermoelectrics_app_2009/wednesday/f leurial.pdf.

[2] H. Kawamoto, "R\&D trends in high efficiency thermoelectric conversion materials for waste heat recovery," Science \& Technology Trends, vol. 30, Sep. 2008, pp. 54-69, Available: http://www.nistep.go.jp/achiev/ftx/eng/stfc/stt030e/qr30pdf/STTqr3004.pdf.

[3] J.-P. Fleurial, T. Caillat, B. Nesmith, R. Ewell, D. Woerner, G. Carr, and L. Jones, "Thermoelectrics: From space power systems to terrestrial waste heat recovery applications," Jan. 2011, Available:

http://www1.eere.energy.gov/vehiclesandfuels/resources/proceedings/2011_thermoelectrics _presentations.html.

[4] C. Vining, "The limited role of thermoelectrics in the climate crisis," May. 2008, Available: http://cvining.com/system/files/articles/vining/presentations/20080501-SummitVining-final.pdf.

[5] D. Kraemer, B. Poudel, H.-P. Feng, J.C. Caylor, B. Yu, X. Yan, Y. Ma, X. Wang, D. Wang, A. Muto, K. McEnaney, M. Chiesa, Z. Ren, and G. Chen, "High-performance flatpanel solar thermoelectric generators with high thermal concentration," Nature Materials, vol. 10, May. 2011, pp. 532-538, dx.doi.org/10.1038/nmat3013.

[6] L.E. Juanicó and G.F. Rinalde, "Comparative analysis of photovoltaic and thermoelectric panels for powering isolated homes," Journal of Renewable and Sustainable Energy, vol. 1, 2009, p. 043107, dx.doi.org/10.1063/1.3180391.

[7] S.K. Bux, R.G. Blair, P.K. Gogna, H. Lee, G. Chen, M.S. Dresselhaus, R.B. Kaner, and J.P. Fleurial, "Nanostructured bulk silicon as an effective thermoelectric material," Advanced Functional Materials, vol. 19, Aug. 2009, pp. 2445-2452, dx.doi.org/10.1002/adfm.200900250.

[8] C.B. Vining, "An inconvenient truth about thermoelectrics," Nature Materials, vol. 8, Feb. 2009, pp. 83-85, dx.doi.org/10.1038/nmat2361.

[9] S.J. Poon, D. Wu, S. Zhu, W. Xie, T.M. Tritt, P. Thomas, and R. Venkatasubramanian, "Half-Heusler phases and nanocomposites as emerging high-ZT thermoelectric materials," Journal of Materials Research, vol. 26, Nov. 2011, pp. 2795-2802, dx.doi.org/10.1557/jmr.2011.329.

[10] E. Toberer, M. Christensen, B. Iversen, and G. Snyder, "High temperature thermoelectric efficiency in Ba8Ga16Ge30," Physical Review B, vol. 77, Feb. 2008, dx.doi.org/10.1103/PhysRevB.77.075203.

[11] G.J. Snyder, "Application of the compatibility factor to the design of segmented and cascaded thermoelectric generators," Applied Physics Letters, vol. 84, 2004, p. 2436, dx.doi.org/10.1063/1.1689396.

[12] P. Martin and L. Olsen, "Scale up of $\mathrm{Si} / \mathrm{Si0} 0 \mathrm{Ge} 0.2$ and B4C/B9C superlattices for harvesting of waste heat in diesel engines," Newport, RI: 2003, Available: www.osti.gov/bridge/servlets/purl/828949-SIhSDq/native/828949.pdf.

[13] T. Caillat, J. Sakamoto, A. Jewell, C.-K. Huang, J. Cheng, J. Paik, P. Gogna, P.-J. Fleurial, and R. Ewell, "Status of skutterudite-based segmented thermoelectric technology 
components development at JPL," JPL TRS 1992+ Available:

http://hdl.handle.net/2014/38757.

[14] A.F. Ioffe, Semiconductor thermoelements and thermoelectric cooling, London, Infosearch, 1957.

[15] T. Hendricks and W.T. Choate, "Engineering scoping study of thermoelectric generator systems for industrial waste heat recovery," Nov. 2006, Available:

http://www1.eere.energy.gov/industry/imf/pdfs/teg_final_report_13.pdf.

[16] J.R. Szczech, J.M. Higgins, and S. Jin, "Enhancement of the thermoelectric properties in nanoscale and nanostructured materials," Journal of Materials Chemistry, vol. 21, 2011, p. 4037, dx.doi.org/10.1039/c0jm02755c.

[17] A. Minnich, H. Lee, X. Wang, G. Joshi, M. Dresselhaus, Z. Ren, G. Chen, and D. Vashaee, "Modeling study of thermoelectric SiGe nanocomposites," Physical Review B, vol. 80, Oct. 2009, dx.doi.org/10.1103/PhysRevB.80.155327.

[18] J.R. Sootsman, D.Y. Chung, and M.G. Kanatzidis, "New and old concepts in thermoelectric materials," Angewandte Chemie International Edition, vol. 48, Nov. 2009, pp. 8616-8639, dx.doi.org/10.1002/anie.200900598.

[19] G.J. Snyder and E.S. Toberer, "Complex thermoelectric materials," Nature Materials, vol. 7, Feb. 2008, pp. 105-114, dx.doi.org/10.1038/nmat2090.

[20] T.M. Tritt, "Thermoelectric phenomena, materials, and applications," Annual Review of Materials Research, vol. 41, Aug. 2011, pp. 433-448, dx.doi.org/10.1146/annurev-matsci062910-100453.

[21] G. Zhu, H. Lee, Y. Lan, X. Wang, G. Joshi, D. Wang, J. Yang, D. Vashaee, H. Guilbert, A. Pillitteri, M. Dresselhaus, G. Chen, and Z. Ren, "Increased phonon scattering by nanograins and point defects in nanostructured silicon with a low concentration of germanium,"

Physical Review Letters, vol. 102, May. 2009, dx.doi.org/10.1103/PhysRevLett.102.196803.

[22] S. Bux, J.-P. Fleurial, R.G. Blair, P.K. Gogna, T. Caillat, and R.B. Kaner, "High temperature thermoelectric properties of nano-bulk silicon and silicon germanium," $M R S$ Proceedings, vol. 1166, Jan. 2011, dx.doi.org/10.1557/PROC-1166-N02-04.

[23] J.P. Dismukes, L. Ekstrom, E.F. Steigmeier, I. Kudman, and D.S. Beers, "Thermal and electrical properties of heavily doped Ge-Si alloys up to $1300^{\circ} \mathrm{K}$," Journal of Applied Physics, vol. 35, 1964, p. 2899, dx.doi.org/10.1063/1.1713126.

[24] G.A. Slack and M.A. Hussain, "The maximum possible conversion efficiency of silicongermanium thermoelectric generators," Journal of Applied Physics, vol. 70, 1991, p. 2694, dx.doi.org/10.1063/1.349385.

[25] S.K. Bux, J.-P. Fleurial, and R.B. Kaner, "Nanostructured materials for thermoelectric applications," Chemical Communications, vol. 46, 2010, p. 8311, dx.doi.org/10.1039/c0cc02627a.

[26] L.A. Stanciu, V.Y. Kodash, and J.R. Groza, "Effects of heating rate on densification and grain growth during field-assisted sintering of $\alpha-\mathrm{Al} 2 \mathrm{O} 3$ and $\mathrm{MoSi} 2$ powders," Metallurgical and Materials Transactions A, vol. 32, Oct. 2001, pp. 2633-2638, dx.doi.org/10.1007/s11661-001-0053-6.

[27] K. Yazawa and A. Shakouri, "Cost-effective waste heat recovery using thermoelectric systems," Journal of Materials Research, vol. 27, Mar. 2012, pp. 1211-1217, dx.doi.org/10.1557/jmr.2012.79. 
[28] C. Vining, "Power for Science and Exploration: Upgrading the General-Purpose Heat Source Radioisotope Thermoelectric Generator (GPHS-RTG)," Nashville, TN: 2010, Available: http://cvining.com/system/files/Vining-JointPropulsionConference-2010.pdf.

[29] C. Bera, M. Soulier, C. Navone, G. Roux, J. Simon, S. Volz, and N. Mingo, "Thermoelectric properties of nanostructured $\mathrm{Si}[\mathrm{sub} 1-\mathrm{x}] \mathrm{Ge}[\mathrm{sub} \mathrm{x}]$ and potential for further improvement," Journal of Applied Physics, vol. 108, 2010, p. 124306, dx.doi.org/10.1063/1.3518579.

[30] I. Takata, "A simple mobility model for electrons and holes," IEEE, , pp. 269-272, dx.doi.org/10.1109/ISPSD.1999.764115.

[31] H.J. Goldsmid, "On the thermal and electrical conductivity of semiconductors," Proceedings of the Physical Society. Section B, vol. 67, Apr. 1954, pp. 360-363, dx.doi.org/10.1088/0370-1301/67/4/412.

[32] E.S. Toberer, A.F. May, and G.J. Snyder, "Zintl chemistry for designing high efficiency thermoelectric materials," Chemistry of Materials, vol. 22, Feb. 2010, pp. 624-634, dx.doi.org/10.1021/cm901956r.

[33] M.A. Green, "Intrinsic concentration, effective densities of states, and effective mass in silicon," Journal of Applied Physics, vol. 67, 1990, p. 2944, dx.doi.org/10.1063/1.345414.

[34] R.E. Jones and S.P. Wesolowski, "Electrical, thermoelectric, and optical properties of strongly degenerate polycrystalline silicon films," Journal of Applied Physics, vol. 56, 1984, p. 1701, dx.doi.org/10.1063/1.334160.

[35] J. Bethin and W.S. Williams, "Ambipolar diffusion contribution to high-temperature thermal conductivity of titanium carbide," Journal of the American Ceramic Society, vol. 60, Sep. 1977, pp. 424-427, dx.doi.org/10.1111/j.1151-2916.1977.tb15526.x.

[36] A. Bulusu and D.G. Walker, "Review of electronic transport models for thermoelectric materials," Superlattices and Microstructures, vol. 44, Jul. 2008, pp. 1-36, dx.doi.org/10.1016/j.spmi.2008.02.008.

[37] C.M. Bhandari and D.M. Rowe, "Boundary scattering of phonons," Journal of Physics C: Solid State Physics, vol. 11, May. 1978, pp. 1787-1794, dx.doi.org/10.1088/00223719/11/9/017.

[38] A.J. Minnich, M.S. Dresselhaus, Z.F. Ren, and G. Chen, "Bulk nanostructured thermoelectric materials: current research and future prospects," Energy \& Environmental Science, vol. 2, 2009, p. 466, dx.doi.org/10.1039/b822664b.

[39] G. Kumar, J. Vandersande, T. Klitsner, R. Pohl, and G. Slack, "Low-temperature heat transport by charge carriers in doped semiconductors," Physical Review B, vol. 31, Feb. 1985, pp. 2157-2162, dx.doi.org/10.1103/PhysRevB.31.2157.

[40] M.D. Ulrich, W.F. Seng, and P.A. Barnes, "Solutions to the Fermi-Dirac integrals in semiconductor physics using polylogarithms," Journal of Computational Electronics, vol. 1, 2002, pp. 431-434, dx.doi.org/10.1023/A:1020784532229.

[41] Z. Bian, M. Zebarjadi, R. Singh, Y. Ezzahri, A. Shakouri, G. Zeng, J.-H. Bahk, J. Bowers, J. Zide, and A. Gossard, "Cross-plane Seebeck coefficient and Lorenz number in superlattices," Physical Review B, vol. 76, Nov. 2007, dx.doi.org/10.1103/PhysRevB.76.205311.

[42] C.B. Vining, "A model for the high-temperature transport properties of heavily doped ntype silicon-germanium alloys," Journal of Applied Physics, vol. 69, 1991, p. 331, dx.doi.org/10.1063/1.347717.

[43] R. Pierret, Semiconductor device fundamentals, Reading Mass.: Addison-Wesley, 1996. 
[44] N.G. Nilsson, "An accurate approximation of the generalized einstein relation for degenerate semiconductors," Physica Status Solidi (a), vol. 19, Sep. 1973, pp. K75-K78, dx.doi.org/10.1002/pssa.2210190159.

[45] M. Cutler, J. Leavy, and R. Fitzpatrick, "Electronic transport in semimetallic cerium sulfide," Physical Review, vol. 133, Feb. 1964, pp. A1143-A1152, dx.doi.org/10.1103/PhysRev.133.A1143.

[46] A. May, E. Toberer, A. Saramat, and G. Snyder, "Characterization and analysis of thermoelectric transport in n-type Ba8Ga16-xGe30+x," Physical Review B, vol. 80, Sep. 2009, dx.doi.org/10.1103/PhysRevB.80.125205.

[47] D. Narducci, E. Selezneva, G. Cerofolini, E. Romano, R. Tonini, and G. Ottaviani, "Opportunities and challenges in the use of heavily doped polycrystalline silicon as a thermoelectric material. An experimental study," Proceedings of the 8th European Conference on Thermoelectrics, Sep. 2010, p. 6, Available: http://hdl.handle.net/10281/17587.

[48] U. Kortshagen and D. Rowe, "UMN/UVA collaboration for thermoelectric devices based on laser sintering of doped SiGe nanoparticles," 2012. 2011, Available: http://www.me.umn.edu/labs/ukgroup/.

[49] J. Martin, L. Wang, L. Chen, and G. Nolas, "Enhanced Seebeck coefficient through energy-barrier scattering in PbTe nanocomposites," Physical Review B, vol. 79, Mar. 2009, dx.doi.org/10.1103/PhysRevB.79.115311.

[50] A. Majumdar, "Thermoelectricity in semiconductor nanostructures," Science, vol. 303, Feb. 2004, pp. 777-778, dx.doi.org/10.1126/science.1093164.

[51] Z. Bian, A. Shakouri, L. Shi, H.-K. Lyeo, and C.K. Shih, "Three-dimensional modeling of nanoscale Seebeck measurements by scanning thermoelectric microscopy," Applied Physics Letters, vol. 87, 2005, p. 053115, dx.doi.org/10.1063/1.2008381.

[52] J.P. Heremans, V. Jovovic, E.S. Toberer, A. Saramat, K. Kurosaki, A. Charoenphakdee, S. Yamanaka, and G.J. Snyder, "Enhancement of thermoelectric efficiency in PbTe by distortion of the electronic density of states," Science, vol. 321, Jul. 2008, pp. 554-557, dx.doi.org/10.1126/science.1159725.

[53] C.J. Vineis, A. Shakouri, A. Majumdar, and M.G. Kanatzidis, "Nanostructured thermoelectrics: big efficiency gains from small features," Advanced Materials, vol. 22, Sep. 2010, pp. 3970-3980, dx.doi.org/10.1002/adma.201000839.

[54] H. Ohta, S. Kim, Y. Mune, T. Mizoguchi, K. Nomura, S. Ohta, T. Nomura, Y. Nakanishi, Y. Ikuhara, M. Hirano, H. Hosono, and K. Koumoto, "Giant thermoelectric Seebeck coefficient of a two-dimensional electron gas in SrTiO3," Nature Materials, vol. 6, Jan. 2007, pp. 129-134, dx.doi.org/10.1038/nmat1821.

[55] F. Blatt, D. Flood, V. Rowe, P. Schroeder, and J. Cox, "Magnon-drag thermopower in iron," Physical Review Letters, vol. 18, Mar. 1967, pp. 395-396, dx.doi.org/10.1103/PhysRevLett.18.395.

[56] T. Geballe and G. Hull, "Seebeck effect in silicon," Physical Review, vol. 98, May. 1955, pp. 940-947, dx.doi.org/10.1103/PhysRev.98.940.

[57] T. Geballe and G. Hull, "Seebeck effect in germanium," Physical Review, vol. 94, Jun. 1954, pp. 1134-1140, dx.doi.org/10.1103/PhysRev.94.1134.

[58] N. Wakeham, A.F. Bangura, X. Xu, J.-F. Mercure, M. Greenblatt, and N.E. Hussey, "Gross violation of the Wiedemann-Franz law in a quasi-one-dimensional conductor," Nature Communications, vol. 2, Jul. 2011, p. 396, dx.doi.org/10.1038/ncomms1406. 
[59] W.-S. Liu, B.-P. Zhang, J.-F. Li, H.-L. Zhang, and L.-D. Zhao, "Enhanced thermoelectric properties in $\mathrm{CoSb}[\mathrm{sub} 3-\mathrm{x}] \mathrm{Te}[\mathrm{sub} \mathrm{x}]$ alloys prepared by mechanical alloying and spark plasma sintering," Journal of Applied Physics, vol. 102, 2007, p. 103717 , dx.doi.org/10.1063/1.2815671.

[60] J. Garg, "Thermal conductivity from first-principles in bulk, disordered, and nanostructured materials," MIT, 2011, Available: http://dspace.mit.edu/handle/1721.1/65280.

[61] Z. Zamanipour, X. Shi, A.M. Dehkordi, J.S. Krasinski, and D. Vashaee, "The effect of synthesis parameters on transport properties of nanostructured bulk thermoelectric p-type silicon germanium alloy," physica status solidi (a), Jun. 2012, dx.doi.org/10.1002/pssa.201228102.

[62] R.P. Chasmar and R. Stratton, "Thermoelectric figure of merit and its relation to thermoelectric generators," Journal of Electronics and Control, vol. 7, 1959, pp. 52-72, dx.doi.org/10.1080/00207215908937186.

[63] R. Simon, "Thermoelectric figure of merit of two-band semiconductors," Journal of Applied Physics, vol. 33, 1962, p. 1830, dx.doi.org/10.1063/1.1728842.

[64] B.A. Cook, J.L. Harringa, S.H. Han, and C.B. Vining, "Si80Ge20 thermoelectric alloys prepared with GaP additions," Journal of Applied Physics, vol. 78, 1995, p. 5474, dx.doi.org/10.1063/1.359663.

[65] X. Ya-Dong, X. Gui-Ying, L. Yan-Hong, and G. Chang-Chun, "Enhancement in Thermoelectrical Power Factor of N-Type Si80Ge20 Alloys," Chinese Physics Letters, vol. 25, Jul. 2008, pp. 2664-2666, dx.doi.org/10.1088/0256-307X/25/7/091.

[66] R. Zhao, L. Shen, and F. Guo, "Enhanced electrical conductivity in Si80Ge20B0.6 alloys with Er addition prepared by spark plasma sintering," Journal of Materials Research, vol. 26, Jun. 2011, pp. 1879-1885, dx.doi.org/10.1557/jmr.2011.127.

[67] A.S. Henry and G. Chen, "Spectral phonon transport properties of silicon based on molecular dynamics simulations and lattice dynamics" Available: http://web.mit.edu/nanoengineering/publications/PDFs/Henry_JCompTheoNanoSci_2008.p df.

[68] J. Garg, N. Bonini, B. Kozinsky, and N. Marzari, "Role of disorder and anharmonicity in the thermal conductivity of silicon-germanium alloys: a first-principles study," Physical Review Letters, vol. 106, Jan. 2011, dx.doi.org/10.1103/PhysRevLett.106.045901.

[69] W. Weber, "Adiabatic bond charge model for the phonons in diamond, Si, Ge, and $\alpha-S n, "$ Physical Review B, vol. 15, May. 1977, pp. 4789-4803, dx.doi.org/10.1103/PhysRevB.15.4789.

[70] N. Mingo, D. Hauser, N.P. Kobayashi, M. Plissonnier, and A. Shakouri, "'Nanoparticle-inalloy' approach to efficient thermoelectrics: Silicides in SiGe," Nano Letters, vol. 9, Feb. 2009, pp. 711-715, dx.doi.org/10.1021/n18031982.

[71] J.B. Haskins, A. Kinaci, and T. Çağin, "Thermal conductivity of Si-Ge quantum dot superlattices," Nanotechnology, vol. 22, Apr. 2011, p. 155701, dx.doi.org/10.1088/09574484/22/15/155701.

[72] A. Shakouri, "Nanoscale energy conversion devices," Jul. 2011, Available: clustertwo.org/Shakouri_COSMOS2011print.pdf.

[73] L.E. Bell, "Cooling, heating, generating power, and recovering waste heat with thermoelectric systems," Science, vol. 321, Sep. 2008, pp. 1457-1461, dx.doi.org/10.1126/science.1158899. 
[74] D. Crane, J. LaGrandeur, and Lon Bell, "Status of segmented element thermoelectric generator for vehicle waste heat recovery," Jan. 2011, Available:

http://www1.eere.energy.gov/vehiclesandfuels/pdfs/thermoelectrics_app_2011/monday/cra ne.pdf.

[75] G. Snyder and T. Ursell, "Thermoelectric efficiency and compatibility," Physical Review Letters, vol. 91, Oct. 2003, dx.doi.org/10.1103/PhysRevLett.91.148301.

[76] D.M. Rowe, Thermoelectrics handbook: macro to nano, Boca Raton: CRC Press, 2006, Available: www.crcpress.com/product/isbn/9780849322648.

[77] G. Joshi, H. Lee, Y. Lan, X. Wang, G. Zhu, D. Wang, R.W. Gould, D.C. Cuff, M.Y. Tang, M.S. Dresselhaus, G. Chen, and Z. Ren, "Enhanced thermoelectric figure-of-merit in nanostructured p-type silicon germanium bulk alloys," Nano Letters, vol. 8, Dec. 2008, pp. 4670-4674, dx.doi.org/10.1021/n18026795.

[78] X.W. Wang, H. Lee, Y.C. Lan, G.H. Zhu, G. Joshi, D.Z. Wang, J. Yang, A.J. Muto, M.Y. Tang, J. Klatsky, S. Song, M.S. Dresselhaus, G. Chen, and Z.F. Ren, "Enhanced thermoelectric figure of merit in nanostructured n-type silicon germanium bulk alloy," Applied Physics Letters, vol. 93, 2008, p. 193121, dx.doi.org/10.1063/1.3027060.

[79] H.J. Goldsmid and A.W. Penn, "Boundary scattering of phonons in solid solutions," Physics Letters A, vol. 27, Sep. 1968, pp. 523-524, dx.doi.org/10.1016/03759601(68)90898-0.

[80] D.M. Rowe and V.S. Shukla, "The effect of phonon-grain boundary scattering on the lattice thermal conductivity and thermoelectric conversion efficiency of heavily doped finegrained, hot-pressed silicon germanium alloy," Journal of Applied Physics, vol. 52, 1981, p. 7421, dx.doi.org/10.1063/1.328733.

[81] K. Biswas, J. He, I.D. Blum, C.-I. Wu, T.P. Hogan, D.N. Seidman, V.P. Dravid, and M.G. Kanatzidis, "High-performance bulk thermoelectrics with all-scale hierarchical architectures," Nature, vol. 489, Sep. 2012, pp. 414-418, dx.doi.org/10.1038/nature11439.

[82] W. Liu, X. Yan, G. Chen, and Z. Ren, "Recent advances in thermoelectric nanocomposites," Nano Energy, Oct. 2011, dx.doi.org/10.1016/j.nanoen.2011.10.001.

[83] M. Zebarjadi, G. Joshi, G. Zhu, B. Yu, A. Minnich, Y. Lan, X. Wang, M. Dresselhaus, Z. Ren, and G. Chen, "Power Factor Enhancement by Modulation Doping in Bulk Nanocomposites," Nano Letters, vol. 11, Jun. 2011, pp. 2225-2230, dx.doi.org/10.1021/nl201206d.

[84] B. Yu, M. Zebarjadi, H. Wang, K. Lukas, H. Wang, D. Wang, C. Opeil, M. Dresselhaus, G. Chen, and Z. Ren, "Enhancement of thermoelectric properties by modulation-doping in silicon germanium alloy nanocomposites," Nano Letters, vol. 12, Apr. 2012, pp. 20772082, dx.doi.org/10.1021/nl3003045.

[85] H. Wang, W. Porter, H. Bottner, J. Konig, L. Chen, S. Bai, T. Tritt, A. Mayolett, J. Senawiratne, C. Smith, F. Harris, J. Sharp, J. Lo, H. Kleinke, and L. Kiss, Annex VIII Thermoelectric Materials For Waste Heat Recovery: An International Collaboration For Transportation Applications, Oak Ridge, TN: Oak Ridge National Laboratory, 2011, Available: http://www.iea-ia-amt.org/reports/topical-reports/reports/topcial-reports/iea-amtornl-tm-report-2011-393/detail.

[86] T.C. Harman, "Special techniques for measurement of thermoelectric properties," Journal of Applied Physics, vol. 29, 1958, p. 1373, dx.doi.org/10.1063/1.1723445. 
[87] M.A. Korzhuev and E.S. Avilov, "Use of the Harman technique for figure of merit measurements of cascade thermoelectric converters," Journal of Electronic Materials, vol. 39, Jun. 2010, pp. 1499-1503, dx.doi.org/10.1007/s11664-010-1301-6.

[88] D.G. Cahill, "Thermal conductivity measurement from 30 to $750 \mathrm{~K}$ : the $3 \omega$ method," Review of Scientific Instruments, vol. 61, 1990, p. 802, dx.doi.org/10.1063/1.1141498.

[89] P.M. Norris and P.E. Hopkins, "Examining Interfacial Diffuse Phonon Scattering Through Transient Thermoreflectance Measurements of Thermal Boundary Conductance," Journal of Heat Transfer, vol. 131, 2009, p. 043207, dx.doi.org/10.1115/1.3072928.

[90] J.-P. Kruth, P. Mercelis, J.V. Vaerenbergh, L. Froyen, and M. Rombouts, "Binding mechanisms in selective laser sintering and selective laser melting," Rapid Prototyping Journal, vol. 11, 2005, pp. 26-36, dx.doi.org/10.1108/13552540510573365.

[91] Y. Gogotsi, Nanomaterials handbook, Boca Raton, FL: CRC Press, 2006, Available: http://books.google.com/books?id=fH_Fs78ZvxsC\&dq.

[92] C.-N. Sun, "Laser sintering of ultra high temperature materials," UVA, 2010, Available: http://gradworks.umi.com/34/36/3436030.html.

[93] G. Schierning, R. Theissmann, H. Wiggers, D. Sudfeld, A. Ebbers, D. Franke, V.T. Witusiewicz, and M. Apel, "Microcrystalline silicon formation by silicon nanoparticles," Journal of Applied Physics, vol. 103, 2008, p. 084305, dx.doi.org/10.1063/1.2903908.

[94] R. Lechner, A.R. Stegner, R.N. Pereira, R. Dietmueller, M.S. Brandt, A. Ebbers, M. Trocha, H. Wiggers, and M. Stutzmann, "Electronic properties of doped silicon nanocrystal films," Journal of Applied Physics, vol. 104, 2008, p. 053701, dx.doi.org/10.1063/1.2973399.

[95] X.Y. Chen, Y.F. Lu, Y.H. Wu, B.J. Cho, B.J. Yang, and T.Y.F. Liew, "Laser annealing of silicon nanocrystal films prepared by pulsed-laser deposition," Journal of Vacuum Science \& Technology B: Microelectronics and Nanometer Structures, vol. 22, 2004, p. 1731, dx.doi.org/10.1116/1.1767829.

[96] T. Baldridge, G. Poling, E. Foroozmehr, R. Kovacevic, T. Metz, V. Kadekar, and M.C. Gupta, "Laser cladding of Inconel 690 on Inconel 600 superalloy for corrosion protection in nuclear applications," Optics and Lasers in Engineering, vol. 51, Feb. 2013, pp. 180-184, dx.doi.org/10.1016/j.optlaseng.2012.08.006.

[97] R.W. Bunce and D.M. Rowe, "The vacuum hot-pressing of germanium and silicongermanium alloys," Journal of Physics D: Applied Physics, vol. 10, Apr. 1977, pp. 941947, dx.doi.org/10.1088/0022-3727/10/6/018.

[98] S. Loughin, D.X. Centurioni, A.G. Robison, J.J. Maley, and J.-P. Fleurial, "High-boron ptype silicon germanium thermoelectric material prepared by the vacuum casting and hot pressing method," AIP, 1993, pp. 747-752, dx.doi.org/10.1063/1.43145.

[99] S.D. Hwang, W.K. Min, I.M. Park, Y.D. Park, Y.S. Kim, and Y.H. Park, "Fabrication and thermoelectric properties of p-type $\mathrm{Si0} 8 \mathrm{Ge} 0.2$ alloy doped with boron," Materials Science Forum, vol. 544-545, 2007, pp. 745-748, dx.doi.org/10.4028/www.scientific.net/MSF.544545.745 .

[100] N. Stein, N. Petermann, R. Theissmann, G. Schierning, R. Schmechel, and H. Wiggers, "Artificially nanostructured n-type SiGe bulk thermoelectrics through plasma enhanced growth of alloy nanoparticles from the gas phase," Journal of Materials Research, vol. 26, Jun. 2011, pp. 1872-1878, dx.doi.org/10.1557/jmr.2011.117.

[101] R. Vilar, "Laser alloying and laser cladding," Materials Science Forum, vol. 301, 1999, pp. 229-252, dx.doi.org/10.4028/www.scientific.net/MSF.301.229. 
[102] S. Bet and A. Kar, "Laser forming of silicon films using nanoparticle precursor," Journal of Electronic Materials, vol. 35, May. 2006, pp. 993-1004, dx.doi.org/10.1007/BF02692559.

[103] L.-P. Scheller, M. Weizman, N.H. Nickel, and B. Yan, "Electrical transport in lasercrystallized polycrystalline silicon-germanium thin-films," Applied Physics Letters, vol. 95, 2009, p. 062101, dx.doi.org/10.1063/1.3194147.

[104] B. Stoib, M.S. Brandt, N. Petermann, H. Wiggers, and M. Stutzmann, "Harman measurements on laser-annealed thin films of Si and Ge nanoparticles," Mar. 2011, Available: http://www.dpg-verhandlungen.de/2011/dresden/ds60.pdf.

[105] H. Wu, B. Hu, N. Tian, and Q. Zheng, "Preparation of $\beta$-FeSi2 thermoelectric material by laser sintering," Materials Letters, vol. 65, Oct. 2011, pp. 2877-2879, dx.doi.org/10.1016/j.matlet.2011.06.061.

[106] T. Baldridge and M.C. Gupta, "Laser hybrid sintering of silicon germanium thermoelectrics," Nov. 2010, Available: www.mrs.org/f10-abstract-11/.

[107] B. Stoib, T. Langmann, S. Matich, T. Antesberger, N. Stein, S. Angst, N. Petermann, R. Schmechel, G. Schierning, D.E. Wolf, H. Wiggers, M. Stutzmann, and M.S. Brandt, "Laser-sintered thin films of doped SiGe nanoparticles," Applied Physics Letters, vol. 100, 2012, p. 231907, dx.doi.org/10.1063/1.4726041.

[108] L. Mangolini, E. Thimsen, and U. Kortshagen, "High-yield plasma synthesis of luminescent silicon nanocrystals," Nano Letters, vol. 5, Apr. 2005, pp. 655-659, dx.doi.org/10.1021/n1050066y.

[109] X.D. Pi and U. Kortshagen, "Nonthermal plasma synthesized freestanding silicongermanium alloy nanocrystals," Nanotechnology, vol. 20, Jul. 2009, p. 295602, dx.doi.org/10.1088/0957-4484/20/29/295602.

[110] X.D. Pi, R. Gresback, R.W. Liptak, S.A. Campbell, and U. Kortshagen, "Doping efficiency, dopant location, and oxidation of Si nanocrystals," Applied Physics Letters, vol. 92, 2008, p. 123102, dx.doi.org/10.1063/1.2897291.

[111] Z.C. Holman and U.R. Kortshagen, "A flexible method for depositing dense nanocrystal thin films: impaction of germanium nanocrystals," Nanotechnology, vol. 21, Aug. 2010, p. 335302, dx.doi.org/10.1088/0957-4484/21/33/335302.

[112] D. Rowe, "Impurities in Silicon Nanocrystals: The intentional and the inherent," University of Minnesota, 2013.

[113] G.L. Bennett, J.J. Lombardo, R.J. Hemler, G. Silverman, C.W. Whitmore, A. Schock, R.W. Zocher, T.K. Keenan, J.C. Hagan, and R.W. Englehart, "Mission of Daring: The General-Purpose Heat Source Radioisotope Thermoelectric Generator," San Diego, CA: AIAA 2006-4096, 2006, Available: http://www.fas.org/nuke/space/gphs.pdf.

[114] R. Lechner, H. Wiggers, A. Ebbers, J. Steiger, M.S. Brandt, and M. Stutzmann, "Thermoelectric effect in laser annealed printed nanocrystalline silicon layers," physica status solidi (RRL) - Rapid Research Letters, vol. 1, Nov. 2007, pp. 262-264, dx.doi.org/10.1002/pssr.200701198.

[115] J.-G. Li, "Wetting of ceramic materials by liquid silicon, aluminium and metallic melts containing titanium and other reactive elements: A review," Ceramics International, vol. 20, Jan. 1994, pp. 391-412, dx.doi.org/10.1016/0272-8842(94)90027-2.

[116] R. Braunstein, A. Moore, and F. Herman, "Intrinsic optical absorption in germaniumsilicon alloys," Physical Review, vol. 109, Feb. 1958, pp. 695-710, dx.doi.org/10.1103/PhysRev.109.695. 
[117] T.J. Lu and N.A. Fleck, "The thermal shock resistance of solids," Acta Materialia, vol. 46, Aug. 1998, pp. 4755-4768, dx.doi.org/10.1016/S1359-6454(98)00127-X.

[118] O. Lindberg, "Hall effect," Proceedings of the IRE, vol. 40, Nov. 1952, pp. 1414-1419, dx.doi.org/10.1109/JRPROC.1952.273972.

[119] Y.C. Chen, S.H. Li, P.K. Bhattacharya, J. Singh, and J.M. Hinckley, "Direct measurement of the Hall factor for holes in relaxed Si1-xGex $(0<\mathrm{x}<1)$," Applied Physics Letters, vol. 64, 1994, p. 3110, dx.doi.org/10.1063/1.111363.

[120] H. Okamoto and T.B. Massalski, "The Au-Si (gold-silicon) system," Bulletin of Alloy Phase Diagrams, vol. 4, Sep. 1983, pp. 190-198, dx.doi.org/10.1007/BF02884878.

[121] H. Okamoto and T.B. Massalski, "The Au-Ge (gold-germanium) system," Bulletin of Alloy Phase Diagrams, vol. 5, Dec. 1984, pp. 601-610, dx.doi.org/10.1007/BF02868323.

[122] D.A. Hite, S.-J. Tang, and P.T. Sprunger, "Reactive epitaxy of beryllium on Si(111)(7×7)," Chemical Physics Letters, vol. 367, Jan. 2003, pp. 129-135, dx.doi.org/10.1016/S0009-2614(02)01637-8.

[123] C.M. Bhandari and D.M. Rowe, "Silicon-germanium alloys as high-temperature thermoelectric materials," Contemporary Physics, vol. 21, May. 1980, pp. 219-242, dx.doi.org/10.1080/00107518008210957.

[124] M. Ronay and R. Schad, "New insight into silicide formation: The creation of silicon self-interstitials," Physical Review Letters, vol. 64, Apr. 1990, pp. 2042-2045, dx.doi.org/10.1103/PhysRevLett.64.2042.

[125] R.W. Olesinski, A.B. Gokhale, and G.J. Abbaschian, "The Ag-Si (silver-silicon) system," Bulletin of Alloy Phase Diagrams, vol. 10, Dec. 1989, pp. 635-640, dx.doi.org/10.1007/BF02877631.

[126] R.W. Olesinski and G.J. Abbaschian, "The Ag-Ge (silver-germanium) system,” Bulletin of Alloy Phase Diagrams, vol. 9, Feb. 1988, pp. 58-64, dx.doi.org/10.1007/BF02877462.

[127] P.E. Hopkins, M. Ding, and J. Poon, "Contributions of electron and phonon transport to the thermal conductivity of GdFeCo and TbFeCo amorphous rare-earth transition-metal alloys," Journal of Applied Physics, vol. 111, 2012, p. 103533, dx.doi.org/10.1063/1.4722231.

[128] A.J. Schmidt, R. Cheaito, and M. Chiesa, "A frequency-domain thermoreflectance method for the characterization of thermal properties," Review of Scientific Instruments, vol. 80, 2009, p. 094901, dx.doi.org/10.1063/1.3212673.

[129] J. Steininger, "Thermodynamics and calculation of the liquidus-solidus gap in homogeneous, monotonic alloy systems," Journal of Applied Physics, vol. 41, 1970, p. 2713, dx.doi.org/10.1063/1.1659286.

[130] G.A. Slack and S.F. Bartram, "Thermal expansion of some diamondlike crystals," Journal of Applied Physics, vol. 46, 1975, p. 89, dx.doi.org/10.1063/1.321373.

[131] T.F. Smith and G.K. White, "The low-temperature thermal expansion and Gruneisen parameters of some tetrahedrally bonded solids," Journal of Physics C: Solid State Physics, vol. 8, Jul. 1975, pp. 2031-2042, dx.doi.org/10.1088/0022-3719/8/13/012.

[132] L.L. Snead, T. Nozawa, Y. Katoh, T.-S. Byun, S. Kondo, and D.A. Petti, "Handbook of SiC properties for fuel performance modeling," Journal of Nuclear Materials, vol. 371, Sep. 2007, pp. 329-377, dx.doi.org/10.1016/j.jnucmat.2007.05.016.

[133] J.P. Dismukes, L. Ekstrom, and R.J. Paff, "Lattice parameter and density in germaniumsilicon alloys," Journal of Physical Chemistry, vol. 68, Oct. 1964, pp. 3021-3027, dx.doi.org/10.1021/j100792a049. 
[134] Y. Touloukian, R. Kirby, R. Taylor, and P. Desai, "Thermophysical properties of matter, vol. 12 - Thermal expansion, metallic elements and alloys" Available:

http://books.google.com/books/about/Thermophysical_Properties_of_Matter_the.html?id=r xc0OAAACAAJ.

[135] H. Matsuokagaya, N. Shoji, and T. Soma, "Specific heat and thermal expansion at high temperatures of Si and Ge," physica status solidi (b), vol. 142, Jul. 1987, pp. K13-K17, dx.doi.org/10.1002/pssb.2221420135.

[136] P.M. Garone, V. Venkataraman, and J.C. Sturn, "Mobility enhancement and quantum mechanical modeling in Ge/sub x/Si/sub 1-x/ channel MOSFETs from 90 to $300 \mathrm{~K}$," IEEE, , pp. 29-32, dx.doi.org/10.1109/IEDM.1991.235431.

[137] F01 Committee, ASTM F723-99: Practice for conversion between resistivity and dopant density for boron-doped, phosphorus-doped, and arsenic-doped silicon, ASTM International, 1999, Available: http://www.astm.org/doiLink.cgi?F723.

[138] D.B. Cuttriss, "Relation between surface concentration and average conductivity in diffused layers in germanium," Bell Syst. Tech. J., vol. 40, 1961, p. 509, Available: http://www.alcatel-lucent.com/bstj/vol40-1961/bstj-vol40-issue02.html.

[139] E. Flage-Larsen and O. Prytz, "The Lorenz function: Its properties at optimum thermoelectric figure-of-merit," Applied Physics Letters, vol. 99, 2011, p. 202108, dx.doi.org/10.1063/1.3656017.

[140] T.-J. King, J.P. McVittie, K.C. Saraswat, and J.R. Pfiester, "Electrical properties of heavily doped polycrystalline silicon-germanium films," IEEE Transactions on Electron Devices, vol. 41, Feb. 1994, pp. 228-232, dx.doi.org/10.1109/16.277374.

[141] S. Krishnamurthy, A. Sher, and A.-B. Chen, "Generalized Brooks' formula and the electron mobility in SixGe1-x alloys," Applied Physics Letters, vol. 47, 1985, p. 160, dx.doi.org/10.1063/1.96248.

[142] N. Petermann, N. Stein, G. Schierning, R. Theissmann, B. Stoib, M.S. Brandt, C. Hecht, C. Schulz, and H. Wiggers, "Plasma synthesis of nanostructures for improved thermoelectric properties," Journal of Physics D: Applied Physics, vol. 44, May. 2011, p. 174034, dx.doi.org/10.1088/0022-3727/44/17/174034.

[143] J.L. Harringa, B.A. Cook, and B.J. Beaudry, "Effects of vial shape on the rate of mechanical alloying in Si80Ge20," Journal of Materials Science, vol. 27, Feb. 1992, pp. 801-804, dx.doi.org/10.1007/BF02403899.

[144] B.J. Winters, J. Holm, and J.T. Roberts, "Thermal processing and native oxidation of silicon nanoparticles," Journal of Nanoparticle Research, vol. 13, Aug. 2011, pp. 54735484, dx.doi.org/10.1007/s11051-011-0535-4.

[145] S. Bowden and C. Honsberg, "Absorption depth," PVEducation.org Available: http://pvcdrom.pveducation.org/SEMICON/ABSDEPTH.HTM.

[146] R. Carlson, "Electrical properties of near-degenerate boron-doped silicon," Physical Review, vol. 100, Nov. 1955, pp. 1075-1078, dx.doi.org/10.1103/PhysRev.100.1075.

[147] C.B. Vining, W. Laskow, J.O. Hanson, R.R. Van der Beck, and P.D. Gorsuch, "Thermoelectric properties of pressure-sintered $\mathrm{Si} 0.8 \mathrm{Ge} 0.2$ thermoelectric alloys," Journal of Applied Physics, vol. 69, 1991, p. 4333, dx.doi.org/10.1063/1.348408.

[148] S.-M. Lee, D.G. Cahill, and R. Venkatasubramanian, "Thermal conductivity of Si-Ge superlattices," Applied Physics Letters, vol. 70, 1997, p. 2957, dx.doi.org/10.1063/1.118755. 
[149] D. Cahill, M. Katiyar, and J. Abelson, "Thermal conductivity of a-Si:H thin films," Physical Review B, vol. 50, Sep. 1994, pp. 6077-6081, dx.doi.org/10.1103/PhysRevB.50.6077.

[150] D. Li, Y. Wu, R. Fan, P. Yang, and A. Majumdar, "Thermal conductivity of Si/SiGe superlattice nanowires," Applied Physics Letters, vol. 83, 2003, p. 3186, dx.doi.org/10.1063/1.1619221.

[151] T. Borca-Tasciuc, W. Liu, J. Liu, T. Zeng, D.W. Song, C.D. Moore, G. Chen, K.L. Wang, M.S. Goorsky, T. Radetic, R. Gronsky, T. Koga, and M.S. Dresselhaus, "Thermal conductivity of symmetrically strained $\mathrm{Si} / \mathrm{Ge}$ superlattices," Superlattices and

Microstructures, vol. 28, Sep. 2000, pp. 199-206, dx.doi.org/10.1006/spmi.2000.0900. 


\section{Appendix}

\section{Publications}

[1] "Thermoelectric films of CW laser sintered doped Si-Ge nanoclusters" [in progress] T. Baldridge, D. Rowe, R. Cheaito, H. Wang, P. Hopkins, U. Kortshagen, and M. C. Gupta, (under preparation).

[2] "CW laser sintering of Si-Ge nanoclusters" [in progress]

T. Baldridge, D. Rowe, M.J. Kirkham, U. Kortshagen, and M. C. Gupta, (under preparation).

[3] "Thermal conductivity of laser sintered $\mathrm{Si}_{0.8} \mathrm{Ge}_{0.2}$ nanoclusters from 80 to 490 K" [in progress]

R. Cheaito, T. Baldridge, D. Rowe, P. Hopkins, U. Kortshagen, and M. C. Gupta, (under preparation).

[4] "Plasma deposition of doped SiGe nanocrystal films: alloy formation and high temperature stability" [in progress]

D. Rowe, T. Baldridge, M.J. Kirkham, M. C. Gupta, and U. Kortshagen, (under preparation).

[5] "Inconel 690 laser cladding on Inconel 600 superalloy for corrosion protection in nuclear applications"

T. Baldridge, G. Poling, E. Foroozmehr, R. Kovacevic, T. Metz, V. Kadekar, and M.C. Gupta, Optics and Lasers in Engineering, v 51, i 2, pp. 180-184.

doi:10.1016/j.optlaseng.2012.08.006

[6] "Fabrication of $\mathrm{ZrB}_{2}-\mathrm{Zr}$ cermet using laser sintering technique"

C. N. Sun, T. Baldridge and M. C. Gupta, Mater. Lett., v 63, pp. 2529-2531 (2009).

doi:10.1016/j.matlet.2009.08.059

[7] "Nanostructures from Zirconium Diboride and Alumina Ceramics"

T. Baldridge, M. C. Gupta, and C. N. Sun, J. Am. Ceram. Soc., v 9, pp. 2891-2896 (2010).

doi:10.1111/j.1551-2916.2010.03781.x

[8] "Zirconium diboride nanofiber generation via microwave arc heating"

T. Baldridge and M. C. Gupta, Nanotechnology, v 19, pp. $275601-275607$ (2008).

doi:10.1088/0957-4484/19/27/275601

\section{Conference Presentations}

[1] T. Baldridge and M.C. Gupta, "Laser hybrid sintering of silicon germanium thermoelectrics", 2010 MRS Fall Meeting Symposium LL, Boston, MA, USA, 2010. LL5.22.

[2] M. C. Gupta, T. Baldridge, and C. N. Sun, "Laser and Electron Beam Sintering of ZrB $\mathrm{Zr}_{2}$-Based Cermets," Materials Science \& Technology, Columbus, OH, USA, 2011. 
[3] T. Baldridge and M. C. Gupta, "Laser + Microwave Hybrid Processing of Ceramic Materials," 9th International Symposium on Laser Precision Microfabrication, Québec, Canada, 2008.

\section{Patent Applications}

[1] M. C. Gupta and T. Baldridge, Zirconium diboride nanofiber generation via microwave arc heating, UVA, 2008. [Provisional]

[2] M. C. Gupta and T. Baldridge, Laser Hybrid Sintering of Silicon Germanium Thermoelectrics, UVA, 2011. [Provisional] 